\title{
Total Synthesis of Caprazamycin A: Practical and Scalable Synthesis of syn- $\beta$-Hydroxyamino Acids and Introduction of a Fatty Acid Side Chain to 1,4-Diazepanone
}

Hugh Nakamura ${ }^{\dagger}$ Chihiro Tsukano, ${ }^{*},{ }^{\dagger}$ Takuma Yoshida ${ }^{\dagger}$ Motohiro Yasui, $^{\dagger}$ Shinsuke Yokouchi, ${ }^{\dagger}$ Yusuke Kobayashi, ${ }^{\dagger}$ Masayuki Igarashi, ${ }^{\dagger}$ and Yoshiji Takemoto*, $^{*}$

${ }^{\dagger}$ Graduate School of Pharmaceutical Sciences, Kyoto University, Yoshida, Sakyo-ku, Kyoto 606-8501, Japan Institute of Microbial Chemistry (BIKAKEN), Tokyo, 3-14-23 Kamiosaki, Shinagawa-ku, Tokyo 141-0021, Japan 


\section{Contents}

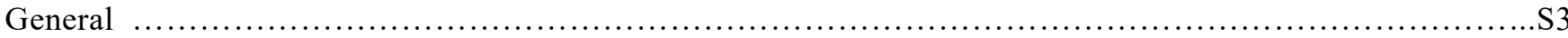

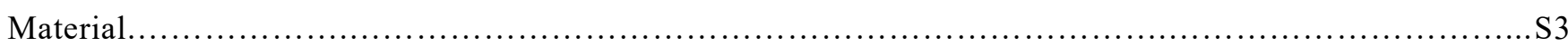

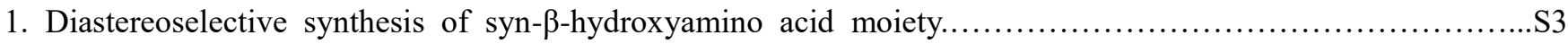

2. Introduction of a fatty-acid side chain using model 1,4-diazepanone …...................................... 15

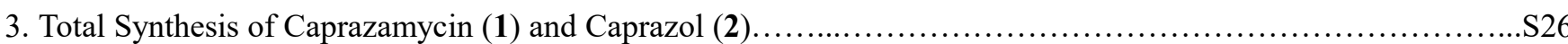

4. Computational study of thiourea catalyzed aldol reaction using benzaldehyde and catalyst 14a using Gaussian 09

5. Computational study of aldol reaction using aldehyde $\mathbf{2 7}$ with isocyanate $\mathbf{1 3}$,

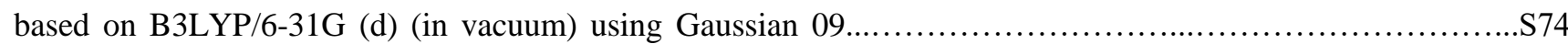

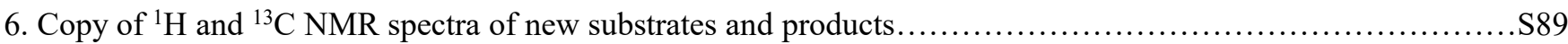

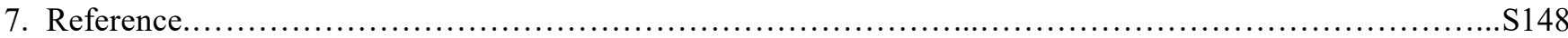


General. All non-aqueous reactions were carried out under a positive atmosphere of argon in dried glassware. Analytical thin-layer chromatography was performed with Silica gel 60 (Merck). Silica gel column chromatography was performed with Kanto silica gel 60 (particle size, 63-210 $\mu \mathrm{m}$ ) and Fuji silysia Chromatorex BW-300. All melting points were determined on YAMAMOTO micro melting point apparatus and are uncorrected. Proton nuclear magnetic resonance ( ${ }^{1} \mathrm{H}$ NMR) spectra were recorded on a JEOL JNM-ECA 500 at $500 \mathrm{MHz}$ or a JEOL JNM-AL 400 at 400 $\mathrm{MHz}$. Chemical shifts are reported relative to $\mathrm{Me}_{4} \mathrm{Si}(\delta 0.0)$ in $\mathrm{CDCl}_{3}$, and residual solvents of DMSO- $\mathrm{d}_{6}(\delta 2.50), \mathrm{D}_{2} \mathrm{O}$ $(\delta 4.79)$ and $\mathrm{CD}_{3} \mathrm{OD}(\delta 3.31)$. Multiplicity is indicated by one or more of the following: s (singlet); $\mathrm{d}$ (doublet); $\mathrm{t}$ (triplet); q (quartet); $\mathrm{m}$ (multiplet); br (broad). Carbon nuclear magnetic resonance $\left({ }^{13} \mathrm{C}\right.$ NMR) spectra were recorded on a JEOL JNM-ECA 500 at $126 \mathrm{MHz}$ or a JEOL JNM-AL 400 at $100 \mathrm{MHz}$. Chemical shifts are reported relative to $\mathrm{CDCl}_{3}\left(\delta\right.$ 77.0), DMSO-d $\mathrm{d}_{6}\left(\delta\right.$ 39.5) and $\mathrm{CD}_{3} \mathrm{OD}(\delta$ 49.0). Infrared spectra were recorded on a FT/IR-4100 Fourier-transform infrared spectrometer ATR (attenuated total reflectance). Low- and high-resolution mass spectra were recorded on JEOL MS700 mass spectrometer for FAB-MS, and Shimadzu LCMS-IT-TOF for ESI-MS.

\section{Material.}

Anhydrous $\mathrm{CH}_{2} \mathrm{Cl}_{2}$, 1,2-dichloroethane, DMF, methanol and $\mathrm{CH}_{3} \mathrm{CN}$ were purchased from KANTO Chemical Co. Aldrich and Wako chemicals. Materials were obtained from Tokyo Chemical Industry Co., Ltd. Aldrich Inc., and other commercial suppliers and used without further purification.

\section{Diastereoselective synthesis of syn- $\beta$-hydroxyamino acid moiety}

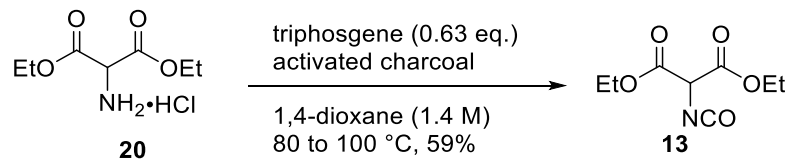

Compound 13: To a mixture of diethyl aminomalonate hydrochloride 20 (3.48 g, $16.5 \mathrm{mmol})$ in 1,4-dioxane (12 mL) were added activated charcoal $(12.1 \mathrm{mg})$ and triphosgene $(3.93 \mathrm{~g}, 13.4 \mathrm{mmol})$ at $0{ }^{\circ} \mathrm{C}$. The reaction mixture was stirred at $80^{\circ} \mathrm{C}$ for $1 \mathrm{~h}$ and then refluxed overnight. The reaction mixture was cooled to room temperature and filtered through celite. The filtrate was concentrated under reduced pressure. The resultant oil was distilled by using Kugelrohr $\left(110^{\circ} \mathrm{C}\right.$, $0.5 \mathrm{mmHg})$ to give the diethyl 2-isocyanatomalonate $13(1.95 \mathrm{~g}, 59 \%)$ as a colorless oil: ${ }^{1} \mathrm{H} \mathrm{NMR}\left(500 \mathrm{MHz}, \mathrm{CDCl}_{3}\right) \delta$ 4.55 (s, 1H), 4.34-4.30 (m, 4H), 1.33 (t, $J=7.1 \mathrm{~Hz}, 6 \mathrm{H}) \mathrm{ppm} ;{ }^{13} \mathrm{C} \mathrm{NMR}\left(126 \mathrm{MHz}, \mathrm{CDCl}_{3}\right) \delta 165.5,129.7,63.5,60.2$, $14.1 \mathrm{ppm}$; IR (ATR) 2253, $1759 \mathrm{~cm}^{-1}$; LRMS (FAB) $202[\mathrm{M}+\mathrm{H}]^{+}$; HRMS (FAB) calcd for $\mathrm{C}_{8} \mathrm{H}_{12} \mathrm{O}_{5} \mathrm{~N}[\mathrm{M}+\mathrm{H}]^{+}$ 202.0715, found: 202.0717 .

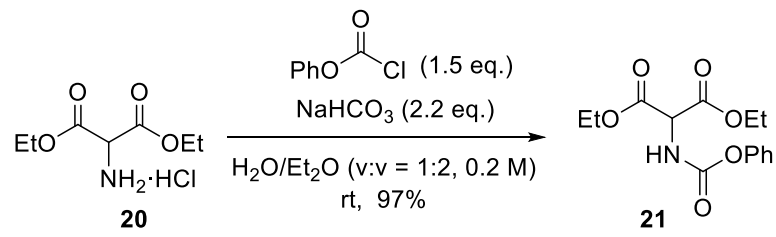

Compound 21: To a solution of diethyl aminomalonate hydrochloride $20(23.2 \mathrm{~g}, 110 \mathrm{mmol})$ in water/ $\mathrm{Et}_{2} \mathrm{O}(550 \mathrm{~mL}$, $\mathrm{v} / \mathrm{v}=1 / 2)$ were added sodium hydrogen carbonate $(20.3 \mathrm{~g}, 242 \mathrm{mmol})$, phenyl chloroformate $(15.3 \mathrm{~mL}, 121 \mathrm{mmol})$, and the reaction mixture was stirred at room temperature. After 1 hour, the resulting mixture was diluted with $\mathrm{CHCl}_{3}$, 
washed with water, brine. The organic layer was dried over $\mathrm{Na}_{2} \mathrm{SO}_{4}$, filtered and concentrated under reduced pressure. Purification by silica gel column chromatography (hexane/EtOAc $=4 / 1$ to 3/7) afforded compound $21(31.4 \mathrm{~g}, 97 \%)$ as yellow oil: ${ }^{1} \mathrm{H}$ NMR $\left(500 \mathrm{MHz}, \mathrm{CDCl}_{3}\right) \delta 7.28(2 \mathrm{H}, \mathrm{t}, J=7.5 \mathrm{~Hz}), 7.13(1 \mathrm{H}, \mathrm{t}, J=7.5 \mathrm{~Hz}), 7.07(2 \mathrm{H}, \mathrm{d}, J=7.5 \mathrm{~Hz})$, $6.08(1 \mathrm{H}, \mathrm{d}, J=7.5 \mathrm{~Hz}), 4.99(1 \mathrm{H}, \mathrm{d}, J=7.5 \mathrm{~Hz}), 4.28-4.17(4 \mathrm{H}, \mathrm{m}), 1.24(6 \mathrm{H}, \mathrm{t}, J=6.5 \mathrm{~Hz}) ;{ }^{13} \mathrm{C} \mathrm{NMR}(125 \mathrm{MHz}$, $\left.\mathrm{CDCl}_{3}\right) \delta 166.1,153.7,150.6,129.3,125.6,121.4,62.7,57.7,13.9 ; \mathrm{IR}\left(\mathrm{ATR}, \mathrm{cm}^{-1}\right) 3359,2984,1734,1484 ; \mathrm{HRMS}$ (ESI) calcd for $\mathrm{C}_{14} \mathrm{H}_{18} \mathrm{NO}_{6}[\mathrm{M}+\mathrm{H}]^{+} 296.1129$; found $\mathrm{m} / z 296.1115$.
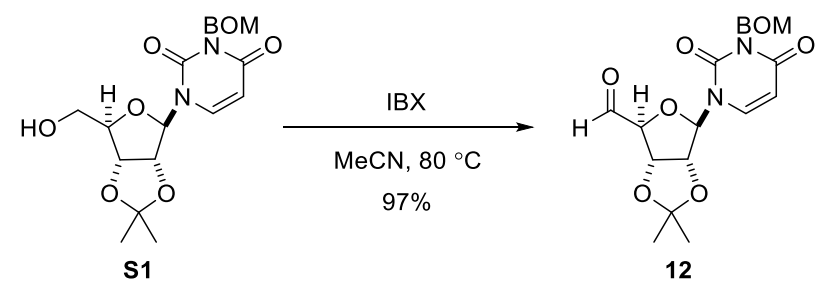

Compound 12: To a solution of alcohol $\mathbf{S 1}^{1}(5.39 \mathrm{~g}, 13.3 \mathrm{mmol})$ in $\mathrm{MeCN}(133 \mathrm{ml})$ was added IBX (5.60 g, 20.0 mmol) at $25{ }^{\circ} \mathrm{C}$ and the resultant solution was then stirred for $1 \mathrm{~h}$ at $80{ }^{\circ} \mathrm{C}$. After cooling to room temperature, the reaction mixture was extracted with EtOAc, washed with brine and dried over $\mathrm{Na}_{2} \mathrm{SO}_{4}$. After concentrated under reduced pressure, purification by silica gel column chromatography and celite (hexane/EtOAc $=1: 4$ ) afforded aldehyde $12(5.19 \mathrm{~g}, 97 \%)$ as an white amorphous: $[\alpha]^{25} \mathrm{D}-15.8^{\circ}\left(c 1.00, \mathrm{CHCl}_{3}\right) ;{ }^{1} \mathrm{H} \mathrm{NMR}\left(500 \mathrm{MHz}, \mathrm{CDCl}_{3}\right) \delta 9.41(1 \mathrm{H}, \mathrm{s})$, 7.37-7.24 (5H, m), $7.19(1 \mathrm{H}, \mathrm{d}, J=8.0 \mathrm{~Hz}), 5.78(1 \mathrm{H}, \mathrm{d}, J=8.0 \mathrm{~Hz}), 5.45(1 \mathrm{H}, \mathrm{s}), 5.40(1 \mathrm{H}, \mathrm{d}, J=10.0 \mathrm{~Hz}), 5.38(1 \mathrm{H}$, d, $J=10.0 \mathrm{~Hz}), 5.24(1 \mathrm{H}, \mathrm{d}, J=6.3 \mathrm{~Hz}), 5.04(1 \mathrm{H}, \mathrm{d}, J=6.3 \mathrm{~Hz}), 4.67(1 \mathrm{H}, \mathrm{d}, J=12.3 \mathrm{~Hz}), 4.64(1 \mathrm{H}, \mathrm{d}, J=12.3 \mathrm{~Hz})$, $4.55(1 \mathrm{H}, \mathrm{br} \mathrm{s}), 1.54(3 \mathrm{H}, \mathrm{s}), 1.37(3 \mathrm{H}, \mathrm{s}) ;{ }^{13} \mathrm{C}$ NMR $\left(126 \mathrm{MHz}, \mathrm{CDCl}_{3}\right) \delta 199.1,162.2,151.3,142.5,137.6,128.3$, $127.8,127.6,113.5,102.5,100.8,94.0,84.8,83.7,72.4,70.2,26.4,24.7$; IR (ATR, $\left.\mathrm{cm}^{-1}\right)$ 1710, 1660, 1451, 1207; HRMS (ESI) calcd for $\mathrm{C}_{20} \mathrm{H}_{23} \mathrm{~N}_{2} \mathrm{O}_{7}[\mathrm{M}+\mathrm{H}]^{+} 403.1500$; found: $m / z 403.1490$.

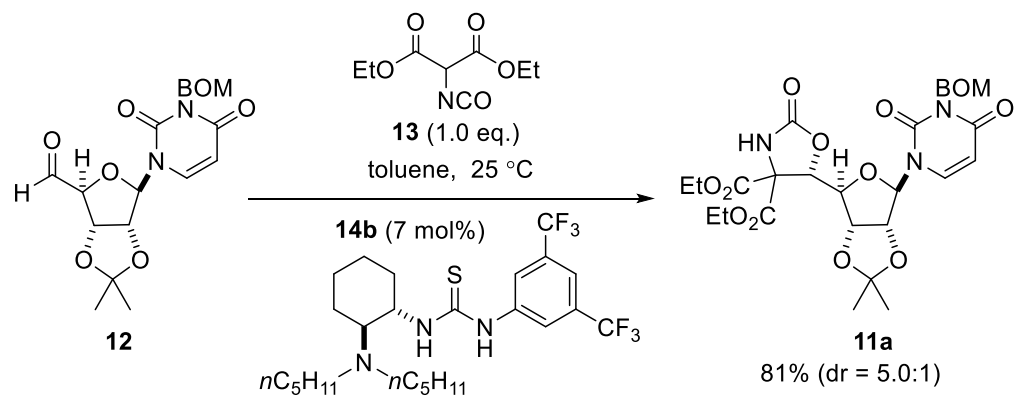

Compound 11a: To a solution of aldehyde $12(78.6 \mathrm{mg}, 195 \mu \mathrm{mol})$ in toluene $(1.0 \mathrm{ml})$ were added $(S, S)-\mathbf{1 4 b}$ (7 mol\%, $7.2 \mathrm{mg}, 13.7 \mu \mathrm{mol})$ and isocyanate $\mathbf{1 3}(39.2 \mathrm{mg}, 195 \mu \mathrm{mol})$ in toluene $(2.9 \mathrm{ml})$ at $25^{\circ} \mathrm{C}$ and the resultant solution was then stirred for $48 \mathrm{~h}$ at $25{ }^{\circ} \mathrm{C}$. The reaction mixture was concentrated under reduced pressure. Purification of the residue by silica gel column chromatography (hexane/EtOAc $=1: 1)$ afforded 11 a $(95.7 \mathrm{mg}, 81 \%, \mathrm{dr}=5.0: 1)$ as an white amorphous: $[\alpha]^{25} \mathrm{D}+116.4^{\circ}\left(c 0.80, \mathrm{CHCl}_{3}\right) ;{ }^{1} \mathrm{H}$ NMR $\left(500 \mathrm{MHz}, \mathrm{CDCl}_{3}\right) \delta$ 7.36-7.29 $(5 \mathrm{H}, \mathrm{m}), 7.21(1 \mathrm{H}, \mathrm{d}, J=$ $8.5 \mathrm{~Hz}), 6.43(1 \mathrm{H}, \mathrm{br} \mathrm{s}), 6.10(1 \mathrm{H}, \mathrm{d}, J=3.5 \mathrm{~Hz}), 5.83(1 \mathrm{H}, \mathrm{d}, J=8.5 \mathrm{~Hz}), 5.48-5.43(3 \mathrm{H}, \mathrm{m}), 4.93(1 \mathrm{H}, \mathrm{dd}, J=6.6$, $3.7 \mathrm{~Hz}), 4.71-4.66(3 \mathrm{H}, \mathrm{m}), 4.54(1 \mathrm{H}, \mathrm{dd}, J=3.7,1.5 \mathrm{~Hz}), 4.33-4.22(4 \mathrm{H}, \mathrm{m}), 1.61(3 \mathrm{H}, \mathrm{s}), 1.37(3 \mathrm{H}, \mathrm{s}), 1.29(3 \mathrm{H}, \mathrm{t}, J$ $=7.2 \mathrm{~Hz}), 1.24(3 \mathrm{H}, \mathrm{t}, J=7.7 \mathrm{~Hz}) ;{ }^{13} \mathrm{C} \mathrm{NMR}\left(126 \mathrm{MHz}, \mathrm{CDCl}_{3}\right) \delta 166.8,166.0,162.9,156.2,151.5,138.4,128.8(2 \mathrm{C})$, 
128.2 (2C), 115.9, 103.7, 91.1, 84.0, 82.5, 80.7, 78.9, 72.7, 71.0, 68.4, 64.19, 64.16, 27.7, 25.9, 13.4, 14.3; IR (ATR, $\mathrm{cm}^{-1}$ ) 3313, 1784, 1745, 1720, 1667; HRMS (ESI) calcd for $\mathrm{C}_{28} \mathrm{H}_{34} \mathrm{~N}_{3} \mathrm{O}_{12}[\mathrm{M}+\mathrm{H}]^{+}$604.2137; found: $m / z$ 604.2141.

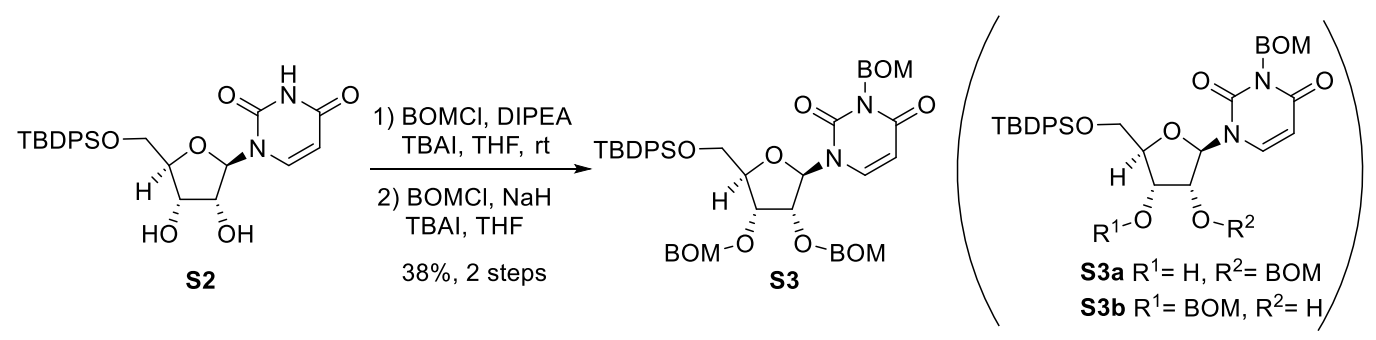

Compound S3: To a solution of diol $\mathbf{S 2}^{2}(6.56 \mathrm{~g}, 14.1 \mathrm{mmol})$ and DIPEA (11.0 mL, $\left.63.3 \mathrm{mmol}\right)$ in THF (100 $\left.\mathrm{mL}\right)$ were added benzyl chloromethyl ether $(8.3 \mathrm{~mL}, 56.2 \mathrm{mmol})$ and tetrabutylammonium iodide $(1.04 \mathrm{~g}, 1.63 \mathrm{mmol})$ at room temperature. The reaction mixture was stirred at room temperature overnight. Then, the mixture was diluted with EtOAc and washed with saturated aqueous $\mathrm{NaHCO}_{3}$ and brine. The solution was dried over $\mathrm{Na}_{2} \mathrm{SO}_{4}$. After filtration and concentration under reduced pressure, the obtained residue was purified by silica gel column chromatography (25-40\% EtOAc/hexane) to give compound $\mathbf{S 3}(1.10 \mathrm{~g}, 9 \%)$ and a mixture of bis-protected alcohols S3a and S3b (3.68 g).

To a solution of the above mixture of S3a and S3b (3.65 g, $5.09 \mathrm{mmol})$ in DMF (50 mL) was added NaH (60\% in mineral oil, $611 \mathrm{mg}, 15.3 \mathrm{mmol})$ at $0{ }^{\circ} \mathrm{C}$ and stirred for $30 \mathrm{~min}$. Then, benzyl chloromethyl ether $(2.3 \mathrm{~mL}, 15.3 \mathrm{mmol})$ and tetrabutylammonium iodide $(188 \mathrm{mg}, 0.509 \mathrm{mmol})$ were added. The reaction solution was stirred at room temperature overnight. The reaction was quenched with water. The mixture was extracted with ether three times. The combine organic layers were washed with brine, dried over $\mathrm{Na}_{2} \mathrm{SO}_{4}$. After filtration and concentration under reduced pressure, the residue was purified by silica gel column chromatography (30-45\% EtOAc/hexane) to give alcohol S3 (3.46 g, 29\%, 2 steps, combined yield $38 \%$ ) as a colorless oil; $[\alpha]_{\mathrm{D}}{ }^{15}+39.7\left(c 1.00, \mathrm{CHCl}_{3}\right) ;{ }^{1} \mathrm{H}$ NMR $(500 \mathrm{MHz}$, $\left.\mathrm{CDCl}_{3}\right) \delta 7.82(\mathrm{~d}, 1 \mathrm{H}, J=8.0 \mathrm{~Hz}), 7.66(\mathrm{~d}, 2 \mathrm{H}, J=6.9 \mathrm{~Hz}), 7.60(\mathrm{~d}, 2 \mathrm{H}, J=6.9 \mathrm{~Hz}), 7.44(\mathrm{dd}, 2 \mathrm{H}, J=7.0,7.0 \mathrm{~Hz})$, 7.40-7.20 (m, 18H), 6.09 (d, 1H, $J=2.9 \mathrm{~Hz}), 5.43(\mathrm{~d}, 1 \mathrm{H}, J=9.5 \mathrm{~Hz}), 5.39$ (d, $1 \mathrm{H}, J=9.5 \mathrm{~Hz}), 5.25(\mathrm{~d}, 1 \mathrm{H}, J=8.3$ Hz), $5.01(\mathrm{~d}, 1 \mathrm{H}, J=6.9 \mathrm{~Hz}), 4.90(\mathrm{~d}, 1 \mathrm{H}, J=6.9 \mathrm{~Hz}), 4.80(\mathrm{dd}, 2 \mathrm{H}, J=11.7,6.9 \mathrm{~Hz}), 4.70(\mathrm{~d}, 2 \mathrm{H}, J=5.7 \mathrm{~Hz}), 4.67$ (s, 2H), 4.64 (s, 2H), 4.59 (s, 2H), 4.37 (dd, 1H, $J=6.3,4.9 \mathrm{~Hz}), 4.33$ (dd, 1H, $J=4.6,3.2 \mathrm{~Hz}), 4.20$ (d, 1H, $J=6.6$ $\mathrm{Hz}), 3.83$ (d, $1 \mathrm{H}, J=10.6 \mathrm{~Hz}), 1.09$ (s, 9H); ${ }^{13} \mathrm{C} \mathrm{NMR}\left(126 \mathrm{MHz}, \mathrm{CDCl}_{3}\right) \delta 162.5,150.9,140.8,138.2,137.9,137.3$, 137.2, 135.6, 135.3, 132.6, 132.0, 130.2, 130.1, 128.6, 128.5, 128.4, 128.0, 128.0, 127.8, 127.7, 127.7, 127.6, 127.0, $101.7,94.3,94.1,88.6,82.5,78.5,72.8,72.2,70.2,70.0,69.8,65.4,62.3,27.0,19.3$; HRMS (ESI) calcd for $\mathrm{C}_{49} \mathrm{H}_{55} \mathrm{~N}_{2} \mathrm{O}_{9} \mathrm{Si}[\mathrm{M}+\mathrm{H}]^{+}$843.3671; found: $m / z$ 843.3695.

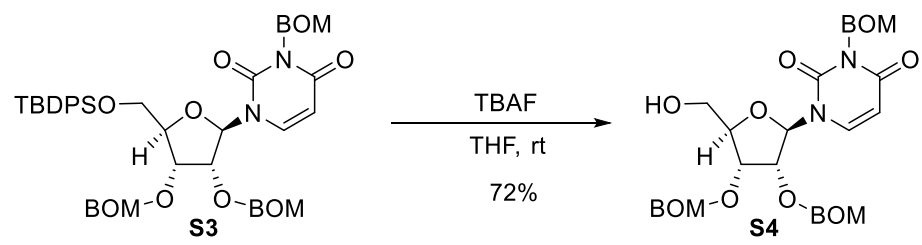

Compound S4: To a solution of compound $\mathbf{S 3}(4.70 \mathrm{~g}, 5.57 \mathrm{mmol})$ in THF $(27.9 \mathrm{~mL})$ was added tetrabutylammonium fluoride (TBAF, 1.0 M THF solution, $6.7 \mathrm{~mL}, 6.7 \mathrm{mmol}$ ). The reaction solution was stirred at room temperature for 
$30 \mathrm{~min}$. The reaction was quenched with water. The mixture was extracted with EtOAc three times. The combined organic layers were washed with brine, dried over $\mathrm{Na}_{2} \mathrm{SO}_{4}$. After filtration and concentration under reduced pressure, the residue was purified by silica gel column chromatography (50-65\% EtOAc/hexane) to give alcohol S4 (2.43 g, $72 \%$ ) as a colorless oil. The compound data was in agreement with previously published report. ${ }^{3}$

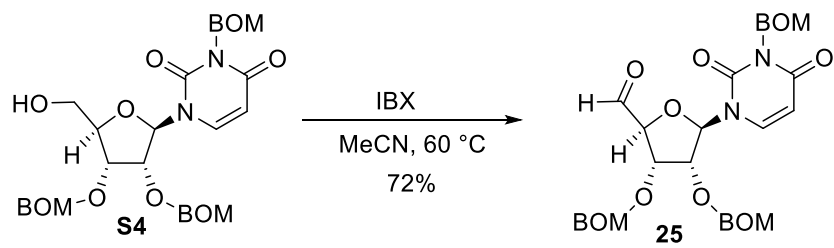

Compound 25: To a solution of $\mathbf{S} 4(1.22 \mathrm{~g}, 2.02 \mathrm{mmol})$ in $\mathrm{CH}_{3} \mathrm{CN}$ was added 2-iodoxybenzoic acid (850 $\mathrm{mg}, 3.04$ mmol). The mixture was stirred at $80{ }^{\circ} \mathrm{C}$ for 2 hours. The resulting mixture was evaporated, dissolved in EtOAc-hexane (1:1), and filtered through $\mathrm{NaHCO}_{3}$ and celite. The filtrate was concentrated and purified by silica gel column chromatography (65-80\% EtOAc/hexane) to afford 25 (892 mg, 72\%) as a colorless oil: ${ }^{1} \mathrm{H}$ NMR (500 MHz, $\left.\mathrm{CDCl}_{3}\right) \delta 9.69(\mathrm{~s}, 1 \mathrm{H}), 7.42(\mathrm{~d}, 1 \mathrm{H}, J=8.0 \mathrm{H}), 7.33-7.26(\mathrm{~m} \mathrm{15H}), 5.90(\mathrm{~d}, 1 \mathrm{H}, J=4.0 \mathrm{~Hz}), 5.71(\mathrm{~d}, 1 \mathrm{H}, J=8.6 \mathrm{~Hz})$, $5.42(\mathrm{~d}, 1 \mathrm{H}, J=10.0 \mathrm{~Hz}), 5.38(\mathrm{~d}, 1 \mathrm{H}, 9.7 \mathrm{~Hz}), 4.91-4.81(\mathrm{~m}, 4 \mathrm{H}), 4.69-4.55(\mathrm{~m}, 7 \mathrm{H}), 4.46-4.42(\mathrm{~m}, 2 \mathrm{H}) ;{ }^{13} \mathrm{C} \mathrm{NMR}$ $\left(125 \mathrm{MHz}, \mathrm{CDCl}_{3}\right) \delta 198.9,162.9,140.2,138.4,137.7,137.5,129.2,129.1,128.9,128.7,128.6,128.4,128.3,128.2$, 103.0, 95.4, 93.0, 86.9, 78.5, 75.6, 72.9, 71.1, 70.8, 70.7; IR (ATR, $\mathrm{cm}^{-1}$ ) 3430, 3064, 3032, 2897, 2361, 1718, 1497, $1454,1410,1358,1269,1171,1069,1039,1026,810,739,699$; HRMS (ESI) calcd for $\mathrm{C}_{33} \mathrm{H}_{33} \mathrm{~N}_{2} \mathrm{O}_{9}[\mathrm{M}-\mathrm{H}]^{-6} 61.2192$ found: $m / z 601.2155$.
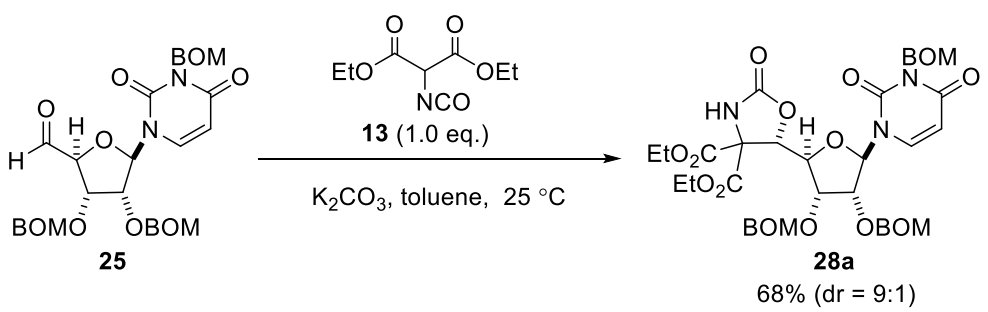

Compound 28a: To the solution of $25(1.88 \mathrm{~g}, 3.13 \mathrm{mmol})$ in dry toluene were added potassium carbonate (186 $\mathrm{mg}$, $1.347 \mathrm{mmol})$ and $\mathbf{1 3}(693 \mathrm{mg})$ at $0{ }^{\circ} \mathrm{C}$. The reaction mixture was stirred at room temperature overnight. The mixture was concentrated and the residue was purified by silica gel column chromatography (30-60\% EtOAc/hexane) to give 28a $(1.71 \mathrm{~g}, 68 \%, \mathrm{dr}=9: 1)$ as a colorless oil: $[\alpha]^{20} \mathrm{D}+91.3^{\circ}\left(c\right.$ 3.70, $\left.\mathrm{CHCl}_{3}\right) ;{ }^{1} \mathrm{H} \mathrm{NMR}\left(400 \mathrm{MHz}, \mathrm{CDCl}_{3}\right) \delta 7.35-7.26$ $(\mathrm{m}, 16 \mathrm{H}), 6.09(\mathrm{~d}, 1 \mathrm{H}, J=2.8 \mathrm{~Hz}), 5.83(\mathrm{~s}, 1 \mathrm{H}), 5.79(\mathrm{~d}, 1 \mathrm{H}, J=8.4 \mathrm{~Hz}), 4.96-4.59(\mathrm{~m}, 10 \mathrm{H}), 4.32-4.23(\mathrm{~m}, 5 \mathrm{H})$, 1.31-1.22 (m, 6H); ${ }^{13} \mathrm{C}$ NMR (100 MHz, $\left.\mathrm{CDCl}_{3}\right) \delta 166.4,165.9,162.5,155.7,151.4,138.2,137.4,137.3,128.9,128.8$, 128.6, 128.4, 128.2, 128.1, 128.0, 127.8, 103.2, 95.0, 94.6, 88.8, 79.4, 77.6, 74.6, 72.5, 70.7, 70.6, 70.3, 67.9, 64.1, 64.0, 14.2; IR (ATR, $\mathrm{cm}^{-1}$ ) 3299, 2979, 2919, 1785, 1748, 1671, 1455, 1364, 1235, 1173, 1090, 889, 742, 700, 629; HRMS (FAB) calcd for $\mathrm{C}_{41} \mathrm{H}_{46} \mathrm{~N}_{3} \mathrm{O}_{14}[\mathrm{M}+\mathrm{H}]^{+}$804.2980; found: $m / z$ 804.2984. 

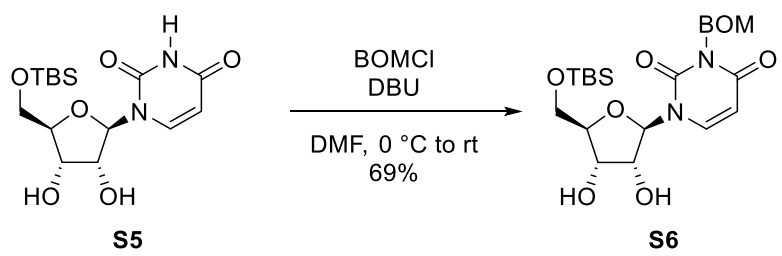

Compound S6: To a solution of 5'-O-tert-butyldimethylsilyl uridine $\mathbf{S 5}^{4}(6.51 \mathrm{~g}, 18.2 \mathrm{mmol})$ in DMF (91 mL, $\left.0.2 \mathrm{M}\right)$ were added DBU $(3.25 \mathrm{~mL}, 20.1 \mathrm{mmol})$ and $\mathrm{BOMCl}(2.76 \mathrm{~mL}, 20.1 \mathrm{mmol})$ at $0{ }^{\circ} \mathrm{C}$ and stirred at $\mathrm{rt}$. After $2 \mathrm{~h}$, the reaction mixture was quenched with $\mathrm{H}_{2} \mathrm{O}$. The mixture was extracted with EtOAc, and washed with brine. The organic layer was dried over with $\mathrm{Na}_{2} \mathrm{SO}_{4}$, After filtration and concentration under reduced pressure, the crude mixture was purified with column chromatography (EtOAc:hexane $=1: 1)$ to give a compound $\mathbf{S 6}(6.05 \mathrm{~g}, 69 \%)$ as a white solid: $[\alpha]^{18} \mathrm{D}+18.7^{\circ}\left(c 1.03, \mathrm{CHCl}_{3}\right) ;{ }^{1} \mathrm{H}$ NMR $\left(500 \mathrm{MHz}, \mathrm{CDCl}_{3}\right) \delta 7.86(\mathrm{~d}, 1 \mathrm{H}, J=8.5 \mathrm{~Hz}), 7.38-7.25(\mathrm{~m}, 5 \mathrm{H}), 5.81(\mathrm{~d}, 1 \mathrm{H}$, $J=4.0 \mathrm{~Hz}), 5.72(\mathrm{~d}, 1 \mathrm{H}, J=8.0 \mathrm{~Hz}), 5.48(\mathrm{~s}, 2 \mathrm{H}), 4.69(\mathrm{~s}, 2 \mathrm{H}), 4.25-4.14(\mathrm{~m}, 3 \mathrm{H}), 3.96-3.93(\mathrm{dd}, 1 \mathrm{H}, J=2.0,11.5 \mathrm{~Hz})$, 3.79-3.77 (dd, $1 \mathrm{H}, J=1.5,11.0 \mathrm{~Hz}), 3.20(\mathrm{~d}, 1 \mathrm{H}, J=4.0 \mathrm{~Hz}), 0.889$ (s, 9H), $0.083(\mathrm{~m}, 6 \mathrm{H}) ;{ }^{13} \mathrm{C}$ NMR $(126 \mathrm{MHz}$, $\left.\mathrm{CDCl}_{3}\right) \delta 162.7,152.1,138.6,137.8,128.5,127.8,101.7,91.8,86.3,76.7,72.5,70.9,70.4,62.6,26.0,18.4,-5.4$, -5.5; HRMS (FAB) calcd for $\mathrm{C}_{23} \mathrm{H}_{35} \mathrm{~N}_{2} \mathrm{O}_{7} \mathrm{Si}[\mathrm{M}+\mathrm{H}]^{+} 479.2214$; found: $m / z 479.2215$.
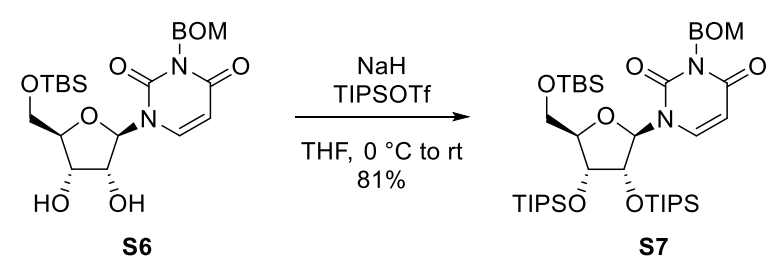

Compound S7: To a solution of compound $\mathbf{S 6}(19.14 \mathrm{~g}, 40.0 \mathrm{mmol})$ in THF (200 mL, $0.2 \mathrm{M})$ was added NaH ( $8.00 \mathrm{~g}$, $200 \mathrm{mmol})$ at $0{ }^{\circ} \mathrm{C}$. After $20 \mathrm{~min}$, TIPSOTf $(29.1 \mathrm{~mL}, 108 \mathrm{mmol})$ was added to the resultant mixture and stirred at $\mathrm{rt}$. After $4 \mathrm{~h}$, the reaction was quenched with $\mathrm{H}_{2} \mathrm{O}$. The mixture was diluted with EtOAc. The combined organic layers were washed with brine, and dried over with $\mathrm{Na}_{2} \mathrm{SO}_{4}$. After filtration and concentration under reduced pressure, the resultant residue was purified with column chromatography (EtOAc:hexane $=1: 4$ ) to give compound $\mathbf{S} 7(25.79 \mathrm{~g}$, $81 \%$ ) as a colorless oil: $[\alpha]^{18} \mathrm{D}+14.5^{\circ}\left(c 0.96, \mathrm{CHCl}_{3}\right) ;{ }^{1} \mathrm{H} \mathrm{NMR}\left(500 \mathrm{MHz}, \mathrm{CDCl}_{3}\right) \delta 7.95(\mathrm{~d}, 1 \mathrm{H}, J=8.5 \mathrm{~Hz})$, 7.38-7.25 (m, 5H), $6.08(\mathrm{~d}, 1 \mathrm{H}, J=4.5 \mathrm{~Hz}), 5.73(\mathrm{~d}, 1 \mathrm{H}, J=8.5 \mathrm{~Hz}), 5.50(\mathrm{~d}, 1 \mathrm{H}, J=10.0 \mathrm{~Hz}), 5.46(\mathrm{~d}, 1 \mathrm{H}, J=9.5 \mathrm{~Hz})$, $4.68(\mathrm{~s}, 2 \mathrm{H}), 4.32(\mathrm{~m}, 2 \mathrm{H}), 4.10(\mathrm{~m}, 1 \mathrm{H}), 3.99-3.96(\mathrm{dd}, 1 \mathrm{H}, J=11.5,2.5 \mathrm{~Hz}), 3.82-3.79$ (dd, $1 \mathrm{H}, J=11.5,2.5 \mathrm{~Hz})$, 1.13-0.99 (m, 42H), 0.12 (s, 3H), 0.11 (s, 3H); ${ }^{13} \mathrm{C}$ NMR (126 MHz, $\left.\mathrm{CDCl}_{3}\right): 163.0,139.4,138.1,128.4,127.8,127.7$, $101.8,88.8,84.7,76.7,72.8,70.3,62.9,31.7,26.2,25.8,18.7,18.3,18.3,18.1,17.8,13.1,12.8,12.4,-3.4,-5.3,-5.4$; HRMS (FAB) calcd for $\mathrm{C}_{41} \mathrm{H}_{75} \mathrm{~N}_{2} \mathrm{O}_{7} \mathrm{Si}_{3}[\mathrm{M}+\mathrm{H}]^{+}$791.4882; found: $\mathrm{m} / z$ 791.4887. 

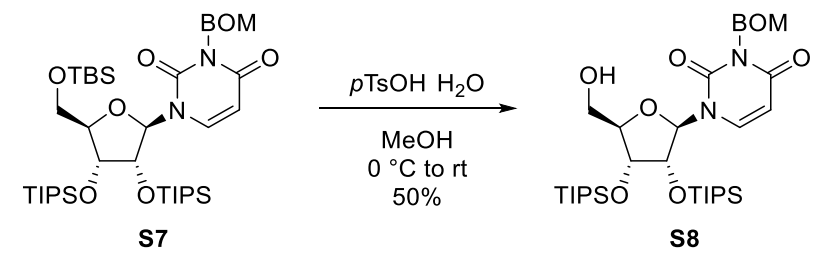

Compound S8: To a solution of compound $\mathbf{S} 7(9.90 \mathrm{~g}, 12.51 \mathrm{mmol})$ in $\mathrm{MeOH}(125 \mathrm{~mL}, 0.1 \mathrm{M})$ at $0{ }^{\circ} \mathrm{C}$, was added $p \mathrm{TsOH} \cdot \mathrm{H}_{2} \mathrm{O}(2.38 \mathrm{~g}, 12.51 \mathrm{mmol})$ in one portion. After the resultant solution was stirred at $\mathrm{rt}$ for $2 \mathrm{~h}$, saturated aqueous $\mathrm{NaHCO}_{3}$ was added to the reaction solution. The mixture was diluted with EtOAc. The combined organic layers were washed with brine, and dried over with $\mathrm{Na}_{2} \mathrm{SO}_{4}$. After filtration and concentration under reduced pressure, the resultant residue was purified with column chromatography (EtOAc:hexane $=3: 2)$ to give compound $\mathbf{S 8}(4.27 \mathrm{~g}$, $50 \%$ ) as a colorless oil: $[\alpha]^{19} \mathrm{D}-32.5^{\circ}\left(c 0.99, \mathrm{CHCl}_{3}\right) ;{ }^{1} \mathrm{H}$ NMR $\left(500 \mathrm{MHz}, \mathrm{CDCl}_{3}\right) \delta 7.51(\mathrm{~d}, 1 \mathrm{H}, J=8.0 \mathrm{~Hz})$, 7.38-7.26 (m, 5H), $5.78(\mathrm{~d}, 1 \mathrm{H}, J=8.0 \mathrm{~Hz}), 5.56(\mathrm{~d}, 1 \mathrm{H}, J=6.5 \mathrm{~Hz}), 5.46(\mathrm{~m}, 2 \mathrm{H}), 4.91-4.89$ (dd, $1 \mathrm{H}, J=6.5,4.0 \mathrm{~Hz})$, $4.68(\mathrm{~s}, 2 \mathrm{H}), 4.41-4.40(\mathrm{dd}, 1 \mathrm{H}, J=4.0,2.0 \mathrm{~Hz}), 4.13(\mathrm{~d}, 1 \mathrm{H}, J=2.0 \mathrm{~Hz}), 3.93-3.90(\mathrm{~m}, 1 \mathrm{H}), 3.77-3.73(\mathrm{~m}, 1 \mathrm{H})$, 3.13-3.11 (m, 1H), 1.11-1.00 (m, 42H); ${ }^{13} \mathrm{C}$ NMR (126 MHz, $\left.\mathrm{CDCl}_{3}\right) \delta 162.8,151.4,142.3,141.0,137.9,128.7,128.4$, $127.8,127.8,127.8,127.1,102.2,94.2,86.2,74.0,73.9,72.2,70.3,65.5,62.5,18.3,18.2,18.1,18.0,13.1,12.7$; HRMS (FAB) calcd for $\mathrm{C}_{35} \mathrm{H}_{61} \mathrm{~N}_{2} \mathrm{O}_{7} \mathrm{Si}_{2}[\mathrm{M}+\mathrm{H}]^{+}$677.4017; found: $\mathrm{m} / 2677.4015$.
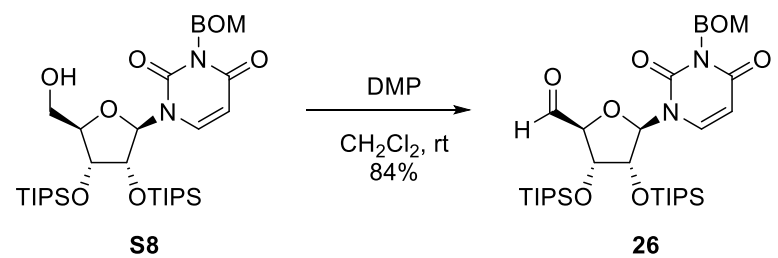

Compound 26: To a solution of compound $\mathbf{S 8}(2.00 \mathrm{~g}, 2.95 \mathrm{mmol})$ in $\mathrm{CH}_{2} \mathrm{Cl}_{2}(30 \mathrm{~mL}, 0.1 \mathrm{M})$ was added Dess-Martin periodinane $(2.50 \mathrm{~g}, 5.89 \mathrm{mmol})$ in one portion. After the reaction solution was stirred at $\mathrm{rt}$ for $3 \mathrm{~h}$, the reaction mixture was filtered with a pad of celite. The filtrate was diluted with $\mathrm{CHCl}_{3}$ and washed with saturated aqueous $\mathrm{NaHCO}_{3}$ and brine. The organic layer was dried over with $\mathrm{Na}_{2} \mathrm{SO}_{4}$. After filtration and concentration under reduced pressure, the resultant residue was purified with column chromatography (EtOAc:hexane $=1: 4)$ to give a compound $26(1.67 \mathrm{~g}$, 84\%) as a colorless oil: $[\alpha]^{18} \mathrm{D}-13.3^{\circ}\left(c 0.49, \mathrm{CHCl}_{3}\right) ;{ }^{1} \mathrm{H} \mathrm{NMR}\left(500 \mathrm{MHz}, \mathrm{CDCl}_{3}\right) \delta 9.86(\mathrm{~d}, J=0.7 \mathrm{~Hz}, 1 \mathrm{H}), 7.60(\mathrm{~d}$, $J=8.1 \mathrm{~Hz}, 1 \mathrm{H}), 7.39-7.30(\mathrm{~m}, 5 \mathrm{H}), 5.84(\mathrm{~d}, J=7.6 \mathrm{~Hz}, 1 \mathrm{H}), 5.51-5.42(\mathrm{~m}, 2 \mathrm{H}), 4.70-4.65(\mathrm{~m}, 3 \mathrm{H}), 4.62-4.57$ $(\mathrm{m}, 1 \mathrm{H}), 4.55(\mathrm{~s}, 2 \mathrm{H}), 1.15-1.05(\mathrm{~m}, 6 \mathrm{H}), 1.04-0.94(\mathrm{~m}, 36 \mathrm{H}) ;{ }^{13} \mathrm{C}$ NMR $\left(126 \mathrm{MHz}, \mathrm{CDCl}_{3}\right) \delta 200.0,162.6,151.0$, 141.1, 128.3, 127.7, 102.6, 93.2, 89.4, 74.4, 73.6, 72.0, 70.6, 31.8, 23.0, 18.1, 18.0, 17.9, 17.7, 14.3, 12.8, 12.5; HRMS (FAB) calcd for $\mathrm{C}_{35} \mathrm{H}_{59} \mathrm{~N}_{2} \mathrm{O}_{7} \mathrm{Si}_{2}[\mathrm{M}+\mathrm{H}]^{+} 675.3861$; found: $m / z$ 675.3868. 

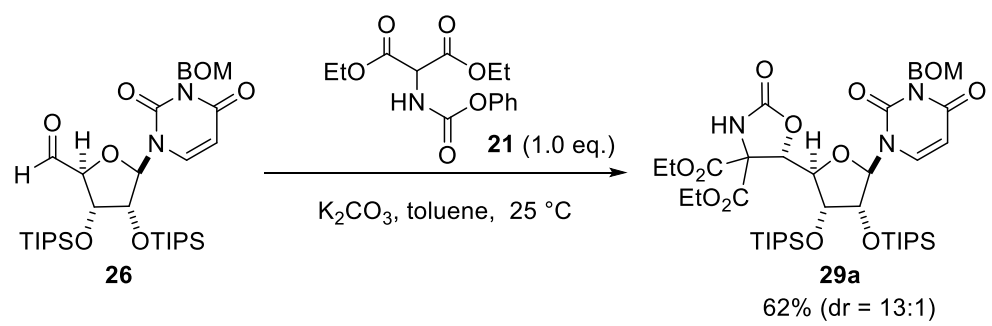

Compound 29a: To the solution of $26(43.0 \mathrm{mg}, 0.0637 \mathrm{mmol})$ in dry toluene $(1 \mathrm{~mL})$ were added $21(20.8 \mathrm{mg}, 0.0704$ $\mathrm{mmol})$ and potassium carbonate $(8.9 \mathrm{mg}, 0.0644 \mathrm{mmol})$ at $0{ }^{\circ} \mathrm{C}$. The reaction mixture was stirred at room temperature for $11 \mathrm{~h}$. The mixture was concentrated under reduced pressure, and the residue was purified by silica gel column chromatography $\left(20-30 \%\right.$ EtOAc/hexane) to give 29 a $(34.4 \mathrm{mg}, 62 \%, \mathrm{dr}=13: 1)$ as a colorless oil: $[\alpha]^{19} \mathrm{D}+71.9^{\circ}(c$ 1.22, $\left.\mathrm{CHCl}_{3}\right) ;{ }^{1} \mathrm{H}$ NMR $\left(500 \mathrm{MHz}, \mathrm{CDCl}_{3}\right) \delta 7.40(\mathrm{~d}, J=8.2 \mathrm{~Hz}, 1 \mathrm{H}), 7.37-7.34(\mathrm{~m}, 2 \mathrm{H}), 7.34-7.28(\mathrm{~m}, 2 \mathrm{H}), 7.28-$ $7.23(\mathrm{~m}, 1 \mathrm{H}), 6.20-6.14(\mathrm{~m}, 2 \mathrm{H}), 5.87(\mathrm{~d}, J=8.2 \mathrm{~Hz}, 1 \mathrm{H}), 5.46(\mathrm{~d}, J=9.8 \mathrm{~Hz}, 1 \mathrm{H}), 5.43(\mathrm{~d}, J=9.8 \mathrm{~Hz}, 1 \mathrm{H}), 5.35(\mathrm{~s}$, $1 \mathrm{H}), 4.65(\mathrm{~s}, 2 \mathrm{H}), 4.48(\mathrm{~d}, J=3.3 \mathrm{~Hz}, 1 \mathrm{H}), 4.39(\mathrm{dd}, J=3.7 \mathrm{~Hz}, 1 \mathrm{H}), 4.36-4.32(\mathrm{~m}, 1 \mathrm{H}), 4.32-4.24(\mathrm{~m}, 3 \mathrm{H}), 4.16-$ $4.09(\mathrm{~m}, 1 \mathrm{H}), 1.29(\mathrm{t}, J=7.1 \mathrm{~Hz}, 3 \mathrm{H}), 1.22(\mathrm{t}, J=7.1 \mathrm{~Hz}, 3 \mathrm{H}), 1.14-1.08(\mathrm{~m}, 22 \mathrm{H}), 1.07-1.03(\mathrm{~m}, 11 \mathrm{H}), 1.03-0.98$ $(\mathrm{m}, 9 \mathrm{H}) ;{ }^{13} \mathrm{C} \mathrm{NMR}\left(126 \mathrm{MHz}, \mathrm{CDCl}_{3}\right) \delta 166.1,165.6,162.5,155.4,151.4,137.8,137.6,128.2,127.7,127.6,102.7$, 87.8, 80.5, 78.0, 74.9, 73.7, 71.9, 70.2, 67.6, 63.8, 63.5, 18.1, 18.0, 17.8, 13.9, 13.7, 12.8, 12.6; HRMS (ESI) calcd for $\mathrm{C}_{43} \mathrm{H}_{70} \mathrm{~N}_{3} \mathrm{O}_{12} \mathrm{Si}_{2}[\mathrm{M}+\mathrm{H}]^{+}$876.4498; found: $m / z$ 876.4493.

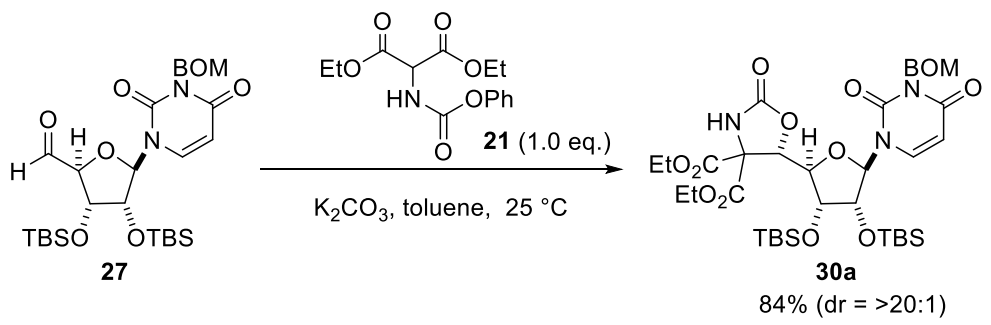

Compound 30a: To a solution of a known aldehyde $27^{5}(16.0 \mathrm{~g}, 27.0 \mathrm{mmol})$ and phenyl carbamate $21(8.80 \mathrm{~g}, 29.8$ $\mathrm{mmol})$ in toluene $(135 \mathrm{~mL})$ was added $\mathrm{K}_{2} \mathrm{CO}_{3}(3.80 \mathrm{~g}, 27.5 \mathrm{mmol})$ at room temperature. After 17 hours, the reaction mixture was filtered with a pad of celite and concentrated under reduced pressure. Purification by silica gel column chromatography (hexane/EtOAc $=4 / 1$ to $1 / 1)$ afforded compound 30a $(17.9 \mathrm{~g}, 84 \%)$ as white amorphous: $[\alpha]^{26}{ }_{\mathrm{D}}$ $+98.1^{\circ}\left(c 1.75, \mathrm{CHCl}_{3}\right) ;{ }^{1} \mathrm{H}$ NMR $\left(500 \mathrm{MHz}, \mathrm{CDCl}_{3}\right) \delta$ 7.43-7.25 (6H, m), $6.07(1 \mathrm{H}, \mathrm{s}), 5.84-5.82(2 \mathrm{H}, \mathrm{m}), 5.48-5.43$ $(2 \mathrm{H}, \mathrm{m}), 5.37(1 \mathrm{H}, \mathrm{s}), 4.70-4.65(2 \mathrm{H}, \mathrm{m}), 4.42-4.20(5 \mathrm{H}, \mathrm{m}), 4.09-4.04(2 \mathrm{H}, \mathrm{m}), 1.34-1.24(6 \mathrm{H}, \mathrm{m}), 0.94(9 \mathrm{H}, \mathrm{s})$, 0.89 (9H, s), 0.15-0.09 (12H, m); ${ }^{13} \mathrm{C}$ NMR $\left(125 \mathrm{MHz}, \mathrm{CDCl}_{3}\right) \delta 166.1,165.6,162.4,155.3,151.0,137.8,137.3$, 128.2, 127.7, 127.6, 102.5, 89.8, 79.9, 77.2, 75.2, 72.1, 71.4, 70.1, 67.6, 63.7, 63.6, 25.8, 25.7, 18.0, 17.9, 13.9, 13.8, $-4.2,-4.5,-4.8,-5.0$; IR (ATR, $\mathrm{cm}^{-1}$ ) 3266, 2932, 2858, 1786, 1750, 1671, 1457, 1258, 840; HRMS (ESI) calcd for $\mathrm{C}_{37} \mathrm{H}_{58} \mathrm{~N}_{3} \mathrm{O}_{12} \mathrm{Si}_{2}[\mathrm{M}+\mathrm{H}]^{+} 792.3554$; found $m / z$. 792.3517. 


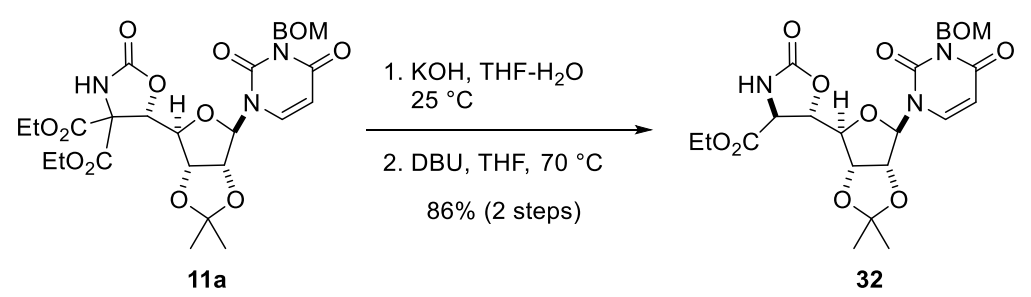

Compound 32: To a solution of 11a $(1.10 \mathrm{~g}, 1.82 \mathrm{mmol})$ in THF $(18 \mathrm{~mL})$ was added $0.25 \mathrm{M} \mathrm{KOH}(8.1 \mathrm{~mL}, 2.00$ mmol) at $25^{\circ} \mathrm{C}$, and the resultant solution was stirred for $0.5 \mathrm{~h}$. The reaction was then quenched with $1 \mathrm{M}$ aqueous $\mathrm{HCl}$. The mixture was extracted with $\mathrm{CHCl}_{3}$, and washed with brine. The organic layer was dried over with $\mathrm{Na}_{2} \mathrm{SO}_{4}$. Filtration and concentration afforded a crude monocarboxylic acid as a white amorphous.

To a solution of the above crude monocarboxylic acid $(1.82 \mathrm{mmol})$ in THF $(18 \mathrm{~mL})$ was added DBU $(135 \mu 1,910$ $\mu \mathrm{mol})$ at $25{ }^{\circ} \mathrm{C}$. The resultant mixture was stirred at $70{ }^{\circ} \mathrm{C}$ for $0.5 \mathrm{~h}$ and then cooled to room temperature. After addition of saturated aqueous $\mathrm{NH}_{4} \mathrm{Cl}$, the mixture was extracted with $\mathrm{CHCl}_{3}$ three times. The combined organic layers were washed with brine and dried over $\mathrm{Na}_{2} \mathrm{SO}_{4}$. After filtration and concentration under reduced pressure, purification by silica gel column chromatography (hexane/EtOAc $=1: 1)$ afforded $32(828 \mathrm{mg}, 86 \%$ for two steps) as a yellow amorphous: $[\alpha]^{25} \mathrm{D}+27.1^{\circ}\left(c 1.01, \mathrm{CHCl}_{3}\right) ;{ }^{1} \mathrm{H} \mathrm{NMR}\left(500 \mathrm{MHz}, \mathrm{CDCl}_{3}\right) \delta$ 7.36-7.28 (5H, m), $7.16(1 \mathrm{H}, \mathrm{d}, J=8.5 \mathrm{~Hz})$, $6.01(1 \mathrm{H}, \mathrm{d}, J=2.9 \mathrm{~Hz}), 5.81(1 \mathrm{H}, \mathrm{d}, J=8.5 \mathrm{~Hz}), 5.49(1 \mathrm{H}, \mathrm{d}, J=9.7 \mathrm{~Hz}), 5.45(1 \mathrm{H}, \mathrm{d}, J=9.7 \mathrm{~Hz}), 5.35(1 \mathrm{H}, \mathrm{br} \mathrm{s})$, $4.98(1 \mathrm{H}, \mathrm{dd}, J=6.4,3.9 \mathrm{~Hz}), 4.93(1 \mathrm{H}, \mathrm{dd}, J=4.9,3.9 \mathrm{~Hz}), 4.83(1 \mathrm{H}, \mathrm{dd}, J=6.4,2.9 \mathrm{~Hz}), 4.70(2 \mathrm{H}, \mathrm{s}), 4.33-4.27$ $(3 \mathrm{H}, \mathrm{m}), 1.60(3 \mathrm{H}, \mathrm{s}), 1.38(3 \mathrm{H}, \mathrm{s}), 1.34(3 \mathrm{H}, \mathrm{t}, J=7.2 \mathrm{~Hz}) ;{ }^{13} \mathrm{C} \mathrm{NMR}\left(126 \mathrm{MHz}, \mathrm{CDCl}_{3}\right) \delta 169.2,162.2,157.3,150.7$, 139.5, 137.6, 128.1, 127.5, 127.4, 115.0, 102.7, 92.3, 85.6, 83.5, 79.6, 77.2, 72.0, 70.2, 62.3, 54.9, 27.0, 25.2, 13.9; IR (ATR, $\mathrm{cm}^{-1}$ ) 3324, 1777, 1717, 1671; HRMS (ESI) calcd for $\mathrm{C}_{25} \mathrm{H}_{30} \mathrm{~N}_{3} \mathrm{O}_{10}[\mathrm{M}+\mathrm{H}]^{+}$532.1926; found: $\mathrm{m} / z$ 532.1931.
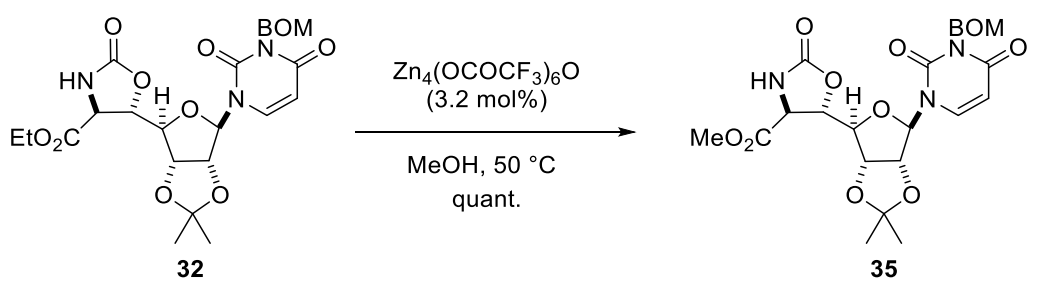

Compound 35: To a solution of $32(3.71 \mathrm{~g}, 6.98 \mathrm{mmol})$ in $\mathrm{MeOH}(70 \mathrm{ml})$ was added $\mathrm{Zn}_{4}\left(\mathrm{OCOCF}_{3}\right)_{6} \mathrm{O}(210 \mathrm{mg}, 220$ $\mu \mathrm{mol})$ at $25{ }^{\circ} \mathrm{C}$, and the resultant solution was then stirred at $50{ }^{\circ} \mathrm{C}$ for $18 \mathrm{~h}$. After cooling to room temperature, the reaction mixture was concentration under reduced pressure. Purification by silica gel column chromatography (hexane/EtOAc $=1: 3)$ afforded 35 (3.62 g, quant.) as a yellow amorphous: $[\alpha]^{25} \mathrm{D}+20.7^{\circ}\left(c 0.26, \mathrm{CHCl}_{3}\right) ;{ }^{1} \mathrm{H}$ NMR $\left(500 \mathrm{MHz}, \mathrm{CDCl}_{3}\right) \delta$ 7.37-7.31 (5H, m), $7.16(1 \mathrm{H}, \mathrm{d}, J=8.3 \mathrm{~Hz}), 6.00(1 \mathrm{H}, \mathrm{d}, J=2.9 \mathrm{~Hz}), 5.81(1 \mathrm{H}, \mathrm{d}, J=8.3 \mathrm{~Hz})$, $5.49(1 \mathrm{H}, \mathrm{d}, J=9.7 \mathrm{~Hz}), 5.45(1 \mathrm{H}, \mathrm{d}, J=9.7 \mathrm{~Hz}), 5.40-5.34(1 \mathrm{H}, \mathrm{m}), 4.97(1 \mathrm{H}, \mathrm{dd}, J=6.6,4.3 \mathrm{~Hz}), 4.93(1 \mathrm{H}, \mathrm{dd}, J=$ $4.3,4.3 \mathrm{~Hz}), 4.84(1 \mathrm{H}, \mathrm{dd}, J=6.6,3.2 \mathrm{~Hz}), 4.70(2 \mathrm{H}, \mathrm{s}), 4.35(1 \mathrm{H}, \mathrm{d}, J=4.9 \mathrm{~Hz}), 4.27(1 \mathrm{H}, \mathrm{dd}, J=4.0,4.0 \mathrm{~Hz}), 3.85$ $(3 \mathrm{H}, \mathrm{s}), 1.61(3 \mathrm{H}, \mathrm{s}), 1.37(3 \mathrm{H}, \mathrm{s}) ;{ }^{13} \mathrm{C} \mathrm{NMR}\left(126 \mathrm{MHz}, \mathrm{CDCl}_{3}\right) \delta 169.6,162.4,157.4,150.8,139.5,137.7,128.2$, 127.64, 127.59, 115.3, 103.0, 92.2, 85.4, 83.6, 79.6, 77.2, 72.2, 70.3, 55.0, 53.2, 27.1, 25.2; IR (ATR, $\mathrm{cm}^{-1}$ ) 3330, 1771, $1669,1456,1091$; HRMS (ESI) calcd for $\mathrm{C}_{24} \mathrm{H}_{27} \mathrm{~N}_{3} \mathrm{O}_{10} \mathrm{Na}[\mathrm{M}+\mathrm{Na}]^{+} 540.1589$; found: $m / z$ 540.1589. 

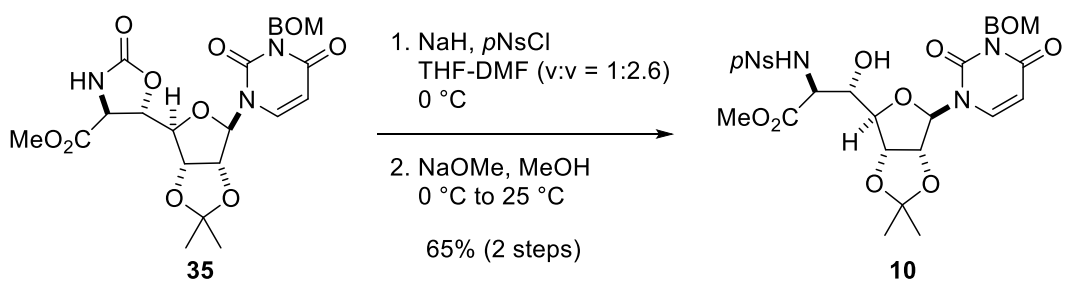

Compound 10: To a solution of $35(1.73 \mathrm{~g}, 3.34 \mathrm{mmol})$ in THF-DMF $(18 \mathrm{ml}, \mathrm{v} / \mathrm{v}=1: 2.6)$ was added $\mathrm{NaH}(60 \%$ in oil, $177 \mathrm{mg}, 4.44 \mathrm{mmol})$ at $0{ }^{\circ} \mathrm{C}$. After the resultant solution was stirred for $40 \mathrm{~min}, p \mathrm{NsCl}(1.22 \mathrm{~g}, 5.50 \mathrm{mmol})$ was added to the reaction mixture at $0{ }^{\circ} \mathrm{C}$. The resultant solution was stirred for $1.5 \mathrm{~h}$. The reaction was then quenched with saturated $\mathrm{NH}_{4} \mathrm{Cl}$. The mixture was extracted with EtOAc. The combined organic layers were washed with brine and dried over $\mathrm{Na}_{2} \mathrm{SO}_{4}$. Filtration and concentration under reduced pressure afforded the crude product as a yellow amorphous.

To a solution of the above crude compound in $\mathrm{MeOH}(25 \mathrm{ml})$ was added $\mathrm{NaOMe}(1.76 \mathrm{~g}, 32.6 \mathrm{mmol})$ at $0{ }^{\circ} \mathrm{C}$, and the resultant solution was then stirred at $25{ }^{\circ} \mathrm{C}$ for $1.5 \mathrm{~h}$. The reaction was quenched with saturated aq. $\mathrm{NH}_{4} \mathrm{Cl}$. After extraction with EtOAc, the combined organic layers were washed with brine and dried over $\mathrm{Na}_{2} \mathrm{SO}_{4}$. After filtration and concentration under reduced pressure, purification by silica gel column chromatography (hexane/EtOAc $=1: 1$ ) afforded 10 (1.46 g, 65\% for two steps) as a yellow amorphous: $[\alpha]^{25} \mathrm{D}+28.1^{\circ}\left(c 1.48, \mathrm{CHCl}_{3}\right) ;{ }^{1} \mathrm{H} \mathrm{NMR}(500 \mathrm{MHz}$, $\left.\mathrm{CDCl}_{3}\right) \delta 8.31(2 \mathrm{H}, \mathrm{d}, J=8.9 \mathrm{~Hz}), 8.01(2 \mathrm{H}, \mathrm{d}, J=8.9 \mathrm{~Hz}), 7.38-7.31(5 \mathrm{H}, \mathrm{m}), 7.18(1 \mathrm{H}, \mathrm{d}, J=8.0 \mathrm{~Hz}), 5.79(1 \mathrm{H}, \mathrm{d}, J$ $=8.0 \mathrm{~Hz}), 5.56(1 \mathrm{H}, \mathrm{d}, J=8.0 \mathrm{~Hz}), 5.47(1 \mathrm{H}, \mathrm{d}, J=9.5 \mathrm{~Hz}), 5.43(1 \mathrm{H}, \mathrm{d}, J=9.5 \mathrm{~Hz}), 5.38(1 \mathrm{H}, \mathrm{d}, J=2.9 \mathrm{~Hz}), 5.08$ $(1 \mathrm{H}, \mathrm{dd}, J=6.6,2.9 \mathrm{~Hz}), 4.98(1 \mathrm{H}, \mathrm{dd}, J=6.6,3.7 \mathrm{~Hz}), 4.69(2 \mathrm{H}, \mathrm{s}), 4.26(1 \mathrm{H}, \mathrm{dd}, J=3.7,3.7 \mathrm{~Hz}), 4.22-4.15(2 \mathrm{H}, \mathrm{m})$, $3.79(1 \mathrm{H}, \mathrm{d}, J=6.5 \mathrm{~Hz}), 3.54(3 \mathrm{H}, \mathrm{s}), 1.55(3 \mathrm{H}, \mathrm{m}), 1.35(3 \mathrm{H}, \mathrm{s}) ;{ }^{13} \mathrm{C} \mathrm{NMR}\left(126 \mathrm{MHz}, \mathrm{CDCl}_{3}\right) \delta 169.7,162.1,151.3$, 150.0, 145.7, 142.0, 137.5, 128.4, 128.4, 127.8, 127.6, 124.2, 114.9, 102.7, 98.0, 85.7, 82.7, 81.5, 72.5, 71.9, 70.3, 58.0, 53.0, 27.2, 25.2; IR (ATR, $\mathrm{cm}^{-1}$ ) 3283, 2988, 1744, 1717, 1663, 1532, 1456, 1350; HRMS (ESI) calcd for $\mathrm{C}_{29} \mathrm{H}_{32} \mathrm{~N}_{4} \mathrm{O}_{13} \mathrm{SNa}[\mathrm{M}+\mathrm{Na}]^{+} 699.1579$; found: $m / z 699.1577$.

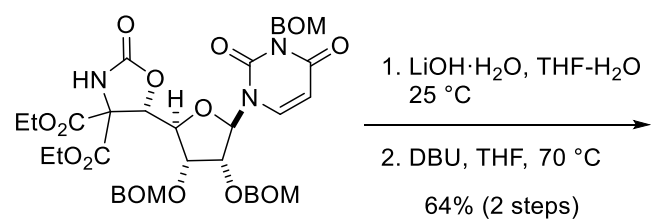

$28 a$

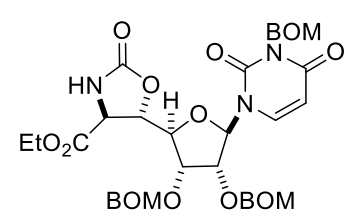

33

Compound 33: To a solution of compound 28a $(1.70 \mathrm{~g}, 2.12 \mathrm{mmol})$ in $\mathrm{THF}-\mathrm{H}_{2} \mathrm{O}(21 \mathrm{~mL}, \mathrm{v} / \mathrm{v}=3: 1)$ was added lithium hydroxide monohydrate $(93.0 \mathrm{mg}, 2.22 \mathrm{mmol})$. The mixture was stirred at room temperature for $1 \mathrm{~h}$. The resulting mixture was diluted with $1 \mathrm{M}$ aqueous $\mathrm{HCl}$ and extracted with $\mathrm{CHCl}_{3}$. The combined organic layers were dried over $\mathrm{Na}_{2} \mathrm{SO}_{4}$. Filtration and concentration under reduced pressure gave the crude monocarboxylic acid (1.67 g). To a solution of the above crude monocarboxylic acid $(1.67 \mathrm{~g})$ in THF $(21 \mathrm{~mL})$ was added DBU $(221 \mu 1,1.48 \mathrm{mmol})$ at $25{ }^{\circ} \mathrm{C}$. The resultant mixture was stirred at $70{ }^{\circ} \mathrm{C}$ for $1 \mathrm{~h}$ and then cooled to room temperature. After addition of saturated aqueous $\mathrm{NH}_{4} \mathrm{Cl}$, the mixture was extracted with $\mathrm{CHCl}_{3}$ three times. The combined organic layers were washed with brine and dried over $\mathrm{Na}_{2} \mathrm{SO}_{4}$. After filtration and concentration under reduced pressure, purification by silica gel column chromatography (50-65\% EtOAt/hexane) afforded 33 (998 mg, 64\% for two steps) as a yellow amorphous: $[\alpha]^{19} \mathrm{D}+66.5^{\circ}\left(c 1.01, \mathrm{CHCl}_{3}\right) ;{ }^{1} \mathrm{H} \mathrm{NMR}\left(500 \mathrm{MHz}, \mathrm{CDCl}_{3}\right) \delta 7.35-7.23(\mathrm{~m}, 16 \mathrm{H}), 6.19(\mathrm{~d}, 1 \mathrm{H}, J=3.7 \mathrm{~Hz})$, 
$5.78(\mathrm{~d}, 1 \mathrm{H}, J=8.3 \mathrm{~Hz}), 5.41(\mathrm{~d}, 1 \mathrm{H}, J=9.7 \mathrm{~Hz}), 5.36(\mathrm{~d}, 1 \mathrm{H}, J=9.7 \mathrm{~Hz}) 4.96(\mathrm{~d}, 1 \mathrm{H}, J=6.9 . \mathrm{Hz}), 4.89-4.84(\mathrm{~m}, 4 \mathrm{H})$, 4.69-4.59 (m, 6H), 4.42 (d, $1 \mathrm{H}, J=14 \mathrm{~Hz}), 4.35-4.20(\mathrm{~m}, 6 \mathrm{H}), 1.29$ (t, 3H, $J=6.9 \mathrm{~Hz}) ;{ }^{13} \mathrm{C}$ NMR $\left(125 \mathrm{MHz}, \mathrm{CDCl}_{3}\right) \delta$ 169.3, 162.5, 157.0, 151.5, 138.2, 137.7, 137.4, 137.3, 128.9, 128.8, 128.6, 128.4, 128.2, 128.1, 128.0, 127.8 103.3, 95.3, 94.9, 88.7, 81.9, 78.0, 76.3, 74.4, 72.5, 70.7, 70.6, 70.4, 63.0, 55.5, 14.4; IR (ATR, $\mathrm{cm}^{-1}$ ) 2978, 2919, 1777, 1670, 1456, 1382, 1234, 1090; HRMS (FAB) calcd for $\mathrm{C}_{38} \mathrm{H}_{42} \mathrm{~N}_{3} \mathrm{O}_{12}[\mathrm{M}+\mathrm{H}]^{+} 732.2768$; found: $m / z$. 732.2770 .
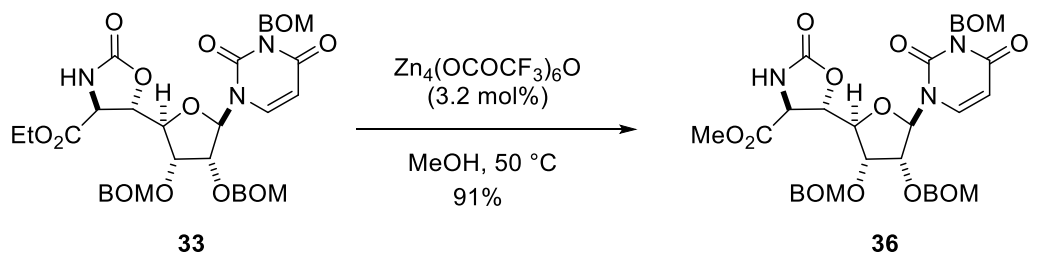

Compound 36: To a solution of 33 (94.0 mg, $0.129 \mathrm{mmol})$ in $\mathrm{MeOH}(2 \mathrm{ml})$ was added $\mathrm{Zn}_{4}\left(\mathrm{OCOCF}_{3}\right)_{6} \mathrm{O}(3.1 \mathrm{mg}, 3.24$ $\mu \mathrm{mol})$ at $25{ }^{\circ} \mathrm{C}$, and the resultant solution was then stirred at $50{ }^{\circ} \mathrm{C}$ for $2 \mathrm{~h}$. After cooling to room temperature, the reaction mixture was concentration under reduced pressure. Purification by silica gel column chromatography (50-65\% EtOAc/hexane) afforded 36 (84.0 mg, 91\%) as a colorless oil: $\left.[\alpha]^{20}{ }_{\mathrm{D}}+80.4^{\circ}(c) 3.85, \mathrm{CHCl}_{3}\right) ;{ }^{1} \mathrm{H}$ NMR $(600$ $\left.\mathrm{MHz}, \mathrm{CDCl}_{3}\right) \delta 7.33-7.23(\mathrm{~m}, 16 \mathrm{H}), 6.20(\mathrm{~d}, 1 \mathrm{H}, J=3.3 \mathrm{~Hz}), 5.94(\mathrm{~s}, 1 \mathrm{H}), 5.81(\mathrm{~d}, 1 \mathrm{H}, J=8.2 \mathrm{~Hz}), 5.43(\mathrm{~d}, 1 \mathrm{H}, J=$ $9.9 \mathrm{~Hz}), 5.38(\mathrm{~d}, 1 \mathrm{H}, J=9.9 \mathrm{~Hz}), 4.98(\mathrm{~d}, 1 \mathrm{H}, J=6.6 \mathrm{~Hz}), \quad 4.91-4.87(\mathrm{~m}, 3 \mathrm{H}), 4.85$ (d, $1 \mathrm{H}, J=5.5 \mathrm{~Hz}), 4.69-4.60(\mathrm{~m}$, $6 \mathrm{H}), 4.46(\mathrm{~d}, 1 \mathrm{H}, J=5.5 \mathrm{~Hz}), 4.36-4.32(\mathrm{~m}, 2 \mathrm{H}), 3.76,(\mathrm{~s}, 3 \mathrm{H}) ;{ }^{13} \mathrm{C}$ NMR $\left(150 \mathrm{MHz}, \mathrm{CDCl}_{3}\right) \delta 153.0,145.8,140.4$, 134.6, 121.3, 120.9, 120.6, 120.5, 112.1, 112.0, 111.8, 111.5, 111.4, 111.3, 111.2, 111.0, 86.4, 78.6, 78.0, 71.9, 65.0, 61.2, 59.4, 57.7, 55.7, 54.0, 53.8, 53.5, 38.7, 36.8; IR (ATR, $\left.\mathrm{cm}^{-1}\right)$ 3312, 3032, 2955, 2900, 1775, 1718, 1666, 1496, 1454, 1358, 1269, 1213, 1173, 1066, 1031; HRMS (FAB) calcd for $\mathrm{C}_{37} \mathrm{H}_{40} \mathrm{~N}_{3} \mathrm{O}_{12}[\mathrm{M}+\mathrm{H}]^{+}$718.2612; found: $\mathrm{m} / \mathrm{z}$ 718.2614.
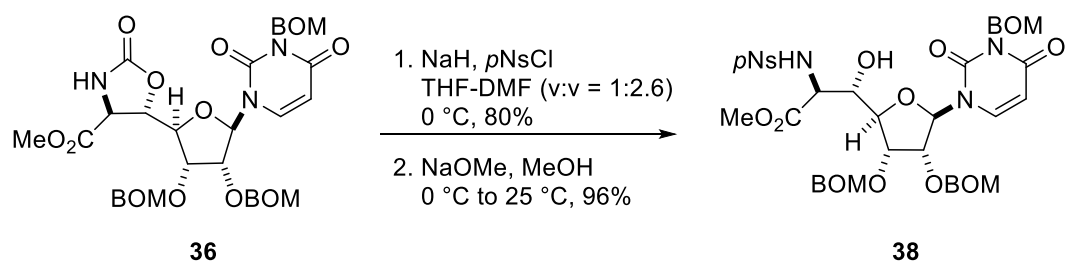

Compound 38: To a solution of 36 ( $365 \mathrm{mg}, 0.509 \mathrm{mmol})$ in THF-DMF $(5 \mathrm{ml}, \mathrm{v} / \mathrm{v}=1: 2.6)$ was added $\mathrm{NaH}(60 \%$ in oil, $24 \mathrm{mg}, 0.610 \mathrm{mmol}$ ) at $0{ }^{\circ} \mathrm{C}$. After the resultant solution was stirred for $30 \mathrm{~min}, p \mathrm{NsCl}$ (135 $\left.\mathrm{mg}, 0.611 \mathrm{mmol}\right)$ was added to the reaction mixture at $0{ }^{\circ} \mathrm{C}$. The resultant solution was stirred for $1 \mathrm{~h}$. The reaction was then quenched with saturated $\mathrm{NH}_{4} \mathrm{Cl}$. The mixture was extracted with EtOAc/Et $2 \mathrm{O}$ three times. The combined organic layers were washed with brine and dried over $\mathrm{Na}_{2} \mathrm{SO}_{4}$. After filtration and concentration under reduced pressure, purification by silica gel column chromatography (40-60\% EtOAc/hexane) afforded a nosylated product S9 (364 mg, 80\%) as a color less oil: $\left.[\alpha]^{20}{ }_{\mathrm{D}}+42.0^{\circ}(c) 3.33, \mathrm{CHCl}_{3}\right) ;{ }^{1} \mathrm{H}$ NMR $\left(500 \mathrm{MHz}, \mathrm{CDCl}_{3}\right) \delta 8.33(\mathrm{~d}, 2 \mathrm{H}, J=8.9 \mathrm{~Hz}), 8.26(\mathrm{~d}, 2 \mathrm{H}, J=8.9 \mathrm{~Hz})$, 7.36-7.24 (m, 15H), $6.86(\mathrm{~d}, 1 \mathrm{H}, J=8.1 \mathrm{~Hz}), 5.96(\mathrm{~d}, 1 \mathrm{H}, J=2.9 \mathrm{~Hz}), 5.42(\mathrm{~d}, 1 \mathrm{H}, J=8.1 \mathrm{~Hz}), 5.40(\mathrm{~s}, 2 \mathrm{H}), 5.06(\mathrm{~d}$, $1 \mathrm{H}, J=3.7 \mathrm{~Hz}), 4.94(\mathrm{~d}, 1 \mathrm{H}, J=7.2 \mathrm{~Hz}), 4.87-4.82(\mathrm{~m}, 4 \mathrm{H}), 4.70-4.59(\mathrm{~m}, 6 \mathrm{H}), 4.35-4.29(\mathrm{~m}, 2 \mathrm{H}), 4.25(\mathrm{~d}, 1 \mathrm{H}, J=$ $6.6 \mathrm{~Hz}), 3.77,(\mathrm{~s}, 3 \mathrm{H}) ;{ }^{13} \mathrm{C} \mathrm{NMR}\left(125 \mathrm{MHz}, \mathrm{CDCl}_{3}\right) \delta 171.5,168.2,162.3,151.4,151.0,150.7,142.6,138.2,138.0$, 
$137.2,137.3,131.1,128.9,128.6,128.4,128.3,128.2,128.0,127.9,124.1,103.1,95.8,95.2,90.6,82.2,78.1,77.6$, 74.9, 74.3, 72.6, 71.0, 70.7, 70.6, 60.7, 59.0, 54.1, 21.4, 14.5; IR (ATR, $\left.\mathrm{cm}^{-1}\right)$ 3107, 3066, 3033, 2959, 2902, 1794 , 1720, 1672, 1606, 1534, 1496, 1453, 1353, 1315, 1266, 1235, 1208, 1180, 1159, 1087, 1031; HRMS (FAB) calcd for $\mathrm{C}_{43} \mathrm{H}_{42} \mathrm{~N}_{4} \mathrm{O}_{16} \mathrm{~S}[\mathrm{M}+\mathrm{H}]^{+}$903.2389; found: $m / z$ 903.2365.

To a solution of the above nosylated product $\mathbf{S 9}(111 \mathrm{mg}, 0.121 \mathrm{mmol})$ in $\mathrm{MeOH}(2.4 \mathrm{ml})$ was added $\mathrm{NaOMe}(65.0$ $\mathrm{mg}, 1.21 \mathrm{mmol}$ ) at $0{ }^{\circ} \mathrm{C}$, and the resultant solution was then stirred for $1 \mathrm{~h}$ at $25^{\circ} \mathrm{C}$. The reaction was quenched with saturated aqueous $\mathrm{NH}_{4} \mathrm{Cl}$. After extraction with EtOAc three times, the combined organic layers were washed with brine and dried over $\mathrm{Na}_{2} \mathrm{SO}_{4}$. After filtration and concentration under reduced pressure, purification by silica gel column chromatography (50-60\% EtOAc/hexane) afforded $38(104 \mathrm{mg}, 96 \%)$ as a colorless oil: $[\alpha]^{20} \mathrm{D}+40.1^{\circ}(c) 3.06$, $\left.\mathrm{CHCl}_{3}\right) ;{ }^{1} \mathrm{H}$ NMR $\left(400 \mathrm{MHz}, \mathrm{CDCl}_{3}\right) \delta 8.27(\mathrm{~d}, 2 \mathrm{H}, J=8.7 \mathrm{~Hz}), 7.97(\mathrm{~d}, 2 \mathrm{H}, J=8.7 \mathrm{~Hz}), 7.40(1 \mathrm{H}, \mathrm{d}, J=8.3 \mathrm{~Hz})$ 7.34-7.22 (m, 15H), $5.95(\mathrm{~d}, 1 \mathrm{H}, J=8.3 \mathrm{~Hz}), 5.68(\mathrm{~d}, 1 \mathrm{H} J=0.5 \mathrm{~Hz}), 5.66(\mathrm{~s}, 1 \mathrm{H}), 5.39-5.33(\mathrm{~m}, 2 \mathrm{H}), 4.89-4.78(\mathrm{~m}$, $4 \mathrm{H}), 4.65-4.52(\mathrm{~m}, 8 \mathrm{H}), 4.38(\mathrm{t}, 1 \mathrm{H} J=5.0 \mathrm{~Hz}), 4.20-4.14(\mathrm{~m}, 2 \mathrm{H}), 4.08-4.04(\mathrm{~m}, 1 \mathrm{H}), 4.00(\mathrm{~d}, 1 \mathrm{H}, J=6.4 \mathrm{~Hz}), 3.78$ $(\mathrm{d}, 1 \mathrm{H}, J=30.0 \mathrm{~Hz}), 3.48(\mathrm{~s}, 3 \mathrm{H}) ;{ }^{13} \mathrm{C}$ NMR $\left(100 \mathrm{MHz}, \mathrm{CDCl}_{3}\right) \delta 170.0,162.6,151.4,150.4,145.8,141.1,137.9$, $137.5,137.2,128.9,128.8,128.7,128.4,128.3 \mathrm{~m} \mathrm{128.0,127.8,} \mathrm{124.5,} \mathrm{102.6,} \mathrm{95.1,} \mathrm{93.3,} \mathrm{82.6,} \mathrm{75.7,} \mathrm{72.6,} \mathrm{71.0,} \mathrm{70.6,}$ 70.3, 58.7, 53.3; IR (ATR, $\mathrm{cm}^{-1}$ ) 3280, 3103, 3033, 2954, 2899 1715, 1662, 1531, 1496, 1455, 1351, 1311, 1269, 1209, 1168, 1070, 1033; HRMS (FAB) calcd for $\mathrm{C}_{42} \mathrm{H}_{45} \mathrm{~N}_{4} \mathrm{O}_{15} \mathrm{~S}[\mathrm{M}+\mathrm{H}]^{+}$877.2602; found: $m / z$ 877.2609.
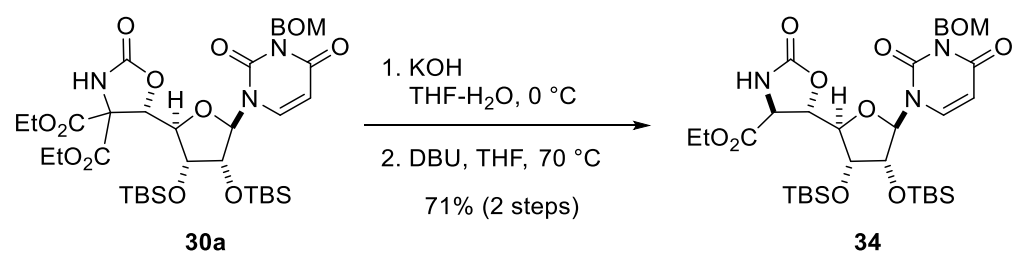

Compound 34: To a solution of compound 30a $(27.5 \mathrm{~g}, 34.7 \mathrm{mmol})$ in THF $(173 \mathrm{~mL}), 0.5 \mathrm{M}$ aq. KOH $(2.73 \mathrm{~g}$ in 83 $\mathrm{mL}$ of $\mathrm{H}_{2} \mathrm{O}, 41.4 \mathrm{mmol}$ ) was added at $0{ }^{\circ} \mathrm{C}$. The reaction mixture was stirred at room temperature for $3 \mathrm{~h}$. Additional aqueous $\mathrm{KOH}\left(686 \mathrm{mg}\right.$ in $20.8 \mathrm{~mL}$ of $\mathrm{H}_{2} \mathrm{O}, 10.4 \mathrm{mmol}$ ) was then added at room temperature and stirred at same temperature. After $20 \mathrm{~min}$, the reaction mixture was cooled to $0{ }^{\circ} \mathrm{C}$ and treated with $1 \mathrm{M}$ aqueous $\mathrm{HCl}$. The reaction mixture was extracted with $\mathrm{CHCl}_{3}$. The combined organic layers were washed with brine and dried over $\mathrm{Na}_{2} \mathrm{SO}_{4}$. Filtration and concentration under reduced pressure afforded a crude carboxylic acid as white amorphous (27.2 $\mathrm{g})$.

To a solution of the above crude carboxylic acid $(27.2 \mathrm{~g})$ in THF $(173 \mathrm{~mL})$ was added DBU $(2.59 \mathrm{~mL}, 17.4 \mathrm{mmol})$ at room temperature, and the resultant solution was warmed to $70{ }^{\circ} \mathrm{C}$ and stirred at $70{ }^{\circ} \mathrm{C}$. After 2.5 hours, the reaction mixture was cooled to $0{ }^{\circ} \mathrm{C}$, and the reaction mixture was treated with aqueous $1 \mathrm{M} \mathrm{HCl}$. After extraction with EtOAc, the combined organic layers were washed with water and brine, and dried over $\mathrm{Na}_{2} \mathrm{SO}_{4}$. After filtration and concentration under reduced pressure, purification by silica gel column chromatography $($ hexane/EtOAc $=4 / 1$ ) afforded compound 34 (17.6 g, 71\% for 2 steps) as white amorphous: $[\alpha]^{27} \mathrm{D}+50.7^{\circ}\left(c 1.28, \mathrm{CHCl}_{3}\right)$; ${ }^{1} \mathrm{H}$ NMR $(500$ $\left.\mathrm{MHz}, \mathrm{CDCl}_{3}\right) \delta 7.41-7.24(6 \mathrm{H}, \mathrm{m}), 6.01(1 \mathrm{H}, \mathrm{d}, J=4.0 \mathrm{~Hz}), 5.84(1 \mathrm{H}, \mathrm{d}, J=8.0 \mathrm{~Hz}), 5.76(1 \mathrm{H}, \mathrm{s}), 5.49-5.45(2 \mathrm{H}, \mathrm{m})$, $4.80(1 \mathrm{H}, \mathrm{d}, J=5.5 \mathrm{~Hz}), 4.68(2 \mathrm{H}, \mathrm{s}), 4.44(1 \mathrm{H}, \mathrm{d}, J=6.5 \mathrm{~Hz}), 4.37-4.22(2 \mathrm{H}, \mathrm{m}), 4.17-4.08(3 \mathrm{H}, \mathrm{m}), 1.32(3 \mathrm{H}, \mathrm{t}, J=$ $8.5 \mathrm{~Hz}), 0.92(9 \mathrm{H}, \mathrm{s}), 0.89(9 \mathrm{H}, \mathrm{s}), 0.12-0.09(12 \mathrm{H}, \mathrm{m}) ;{ }^{13} \mathrm{C} \mathrm{NMR}\left(125 \mathrm{MHz}, \mathrm{CDCl}_{3}\right) \delta 168.9,162.4,156.6,151.2$, $137.8,137.4,128.3,127.7,127.6,102.7,89.1,83.0,75.8,75.0,72.1,71.1,70.2,62.7,55.3,25.8,25.7,18.0,17.9,14.0$, $-4.3,-4.5,-4.7,-4.9$; IR (ATR, $\left.\mathrm{cm}^{-1}\right)$ 3291, 2932, 2858, 1778, 1673, 1455, 838; HRMS (ESI) calcd for 

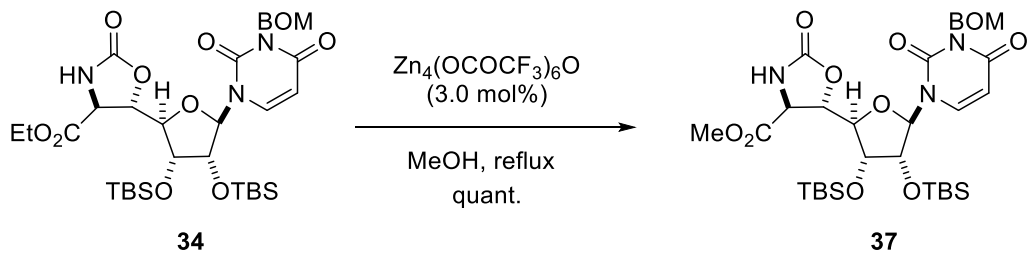

Compound 37: To a solution of compound $34(1.88 \mathrm{~g}, 2.61 \mathrm{mmol})$ in $\mathrm{MeOH}(26 \mathrm{~mL})$ was added $\mathrm{Zn} 4\left(\mathrm{OCOCF}_{3}\right)_{6} \mathrm{O}$ (76.1 $\mathrm{mg}, 79.6 \mu \mathrm{mol}$ ) at room temperature, and the resultant reaction mixture was refluxed. After $2 \mathrm{~h}$, the reaction mixture was cooled to room temperature, and filtered with a pad of celite and silica gel to give compound $\mathbf{3 7}$ (1.86 g, quant.) as white amorphous: $[\alpha]^{27} \mathrm{D}+55.0^{\circ}\left(c\right.$ 1.50, $\left.\mathrm{CHCl}_{3}\right)$; ${ }^{1} \mathrm{H}$ NMR $\left(500 \mathrm{MHz}, \mathrm{CDCl}_{3}\right) \delta 7.43-7.25(6 \mathrm{H}, \mathrm{m}), 6.01$ $(1 \mathrm{H}, \mathrm{d}, J=4.0 \mathrm{~Hz}), 5.86-5.84(2 \mathrm{H}, \mathrm{m}), 5.49-5.43(2 \mathrm{H}, \mathrm{m}), 4.83(1 \mathrm{H}, \mathrm{dd}, J=6.0,1.0 \mathrm{~Hz}), 4.69(2 \mathrm{H}, \mathrm{s}), 4.46(1 \mathrm{H}, \mathrm{d}, J$ $=6.0 \mathrm{~Hz}), 4.16-4.07(3 \mathrm{H}, \mathrm{m}), 3.85(3 \mathrm{H}, \mathrm{s}), 0.93(9 \mathrm{H}, \mathrm{s}), 0.89(9 \mathrm{H}, \mathrm{s}), 0.13-0.09(12 \mathrm{H}, \mathrm{m}) ;{ }^{13} \mathrm{C}$ NMR $(125 \mathrm{MHz}$, $\left.\mathrm{CDCl}_{3}\right) \delta 169.4,162.4,156.7,151.2,137.8,137.4,128.3,127.7,127.6,102.7,89.2,83.0,75.8,75.1,72.1,71.1,70.2$, 55.3, 53.3, 25.8, 25.7, 18.02, 17.96, -4.3, -4.6, -4.7, -4.9; IR (ATR, $\left.\mathrm{cm}^{-1}\right)$ 2955, 2857, 1741, 1674, 1457, 1253, 840; HRMS (ESI) calcd for $\mathrm{C}_{33} \mathrm{H}_{52} \mathrm{~N}_{3} \mathrm{O}_{10} \mathrm{Si}_{2}[\mathrm{M}+\mathrm{H}]^{+}$706.3186; found $\mathrm{m} / z$ 706.3165.
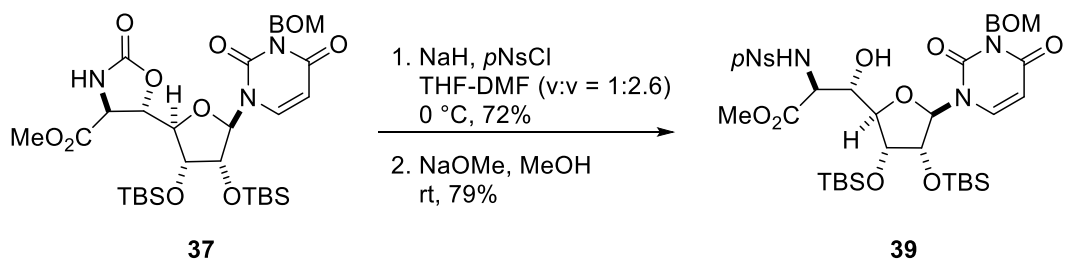

Compound 39: To a solution of compound $37(1.51 \mathrm{~g}, 2.14 \mathrm{mmol})$ in THF/DMF $(21.4 \mathrm{~mL}, \mathrm{v} / \mathrm{v}=1: 2.6)$ was added $\mathrm{NaH}(60 \%$ in oil, $110 \mathrm{mg}, 2.75 \mathrm{mmol})$ at $0{ }^{\circ} \mathrm{C}$, and the resultant solution was stirred for $20 \mathrm{~min}$. To the reaction mixture was then added $p \mathrm{NsCl}(620 \mathrm{mg}, 2.80 \mathrm{mmol})$ at $0{ }^{\circ} \mathrm{C}$, and the resultant solution was stirred at room temperature for $1 \mathrm{~h}$. The reaction was then quenched with water. The mixture was extracted with EtOAc. The combined organic layers were washed with brine and dried over $\mathrm{Na}_{2} \mathrm{SO}_{4}$. After filtration and concentration under reduced pressure, purification by silica gel column chromatography (hexane/EtOAc $=7 / 3)$ afforded a nosylated product $\mathbf{S 1 0}(1.38 \mathrm{~g}$ $72 \%)$ as white amorphous: $[\alpha]^{25}{ }_{\mathrm{D}}+41.8^{\circ}\left(c 1.55, \mathrm{CHCl}_{3}\right) ;{ }^{1} \mathrm{H}$ NMR $\left(500 \mathrm{MHz}, \mathrm{CDCl}_{3}\right) \delta 8.35(2 \mathrm{H}, \mathrm{d}, J=9.0 \mathrm{~Hz}), 8.30$ $(2 \mathrm{H}, \mathrm{d}, J=9.0 \mathrm{~Hz}), 7.38-7.26(5 \mathrm{H}, \mathrm{m}), 7.00(1 \mathrm{H}, \mathrm{d}, J=8.0 \mathrm{~Hz}), 5.88(1 \mathrm{H}, \mathrm{d}, J=2.5 \mathrm{~Hz}), 5.69(1 \mathrm{H}, \mathrm{d}, J=8.0 \mathrm{~Hz})$, 5.49-5.44 (2H, m), $5.11(1 \mathrm{H}, \mathrm{d}, J=4.0 \mathrm{~Hz}), 4.71-4.65(3 \mathrm{H}, \mathrm{m}), 4.16(1 \mathrm{H}, \mathrm{d}, J=5.5 \mathrm{~Hz}), 4.08-4.05(2 \mathrm{H}, \mathrm{m}), 3.92$ $(3 \mathrm{H}, \mathrm{s}), 0.91-0.86(18 \mathrm{H}, \mathrm{m}), 0.13-0.07(12 \mathrm{H}, \mathrm{m}) ;{ }^{13} \mathrm{C} \mathrm{NMR}\left(125 \mathrm{MHz}, \mathrm{CDCl}_{3}\right) \delta 168.1,162.0,151.1,150.8,150.4$, 142.3, 137.9, 137.0, 130.8, 128.3, 127.7, 127.6, 123.8, 102.7, 90.4, 82.9, 75.0, 74.6, 72.1, 70.4, 70.3, 59.0, 53.9, 25.73, 25.72, 17.99, 17.98, -4.2, -4.5, -4.7, -5.0; IR (ATR, $\mathrm{cm}^{-1}$ ) 2932, 2858, 1796, 1728, 1678, 1536, 1355, 839; HRMS (ESI) calcd for $\mathrm{C}_{39} \mathrm{H}_{55} \mathrm{~N}_{4} \mathrm{O}_{14} \mathrm{Si}_{2} \mathrm{~S}[\mathrm{M}+\mathrm{H}]^{+}$891.2969; found $\mathrm{m} / z$ 891.2956.

To a solution of the above nosylated product $\mathbf{S 1 0}(828 \mathrm{mg}, 0.929 \mathrm{mmol})$ in $\mathrm{MeOH}(9.2 \mathrm{~mL})$ was added $\mathrm{NaOMe}$ (255mg, $4.72 \mathrm{mmol}$ ) at $0{ }^{\circ} \mathrm{C}$, and the resultant reaction mixture was stirred at room temperature for $1 \mathrm{~h}$. The reaction mixture was diluted with water, and extracted with EtOAc. The combined organic layers were washed with brine, dried 
over $\mathrm{Na}_{2} \mathrm{SO}_{4}$. After filtration and concentration under reduced pressure, purification by silica gel column chromatography (hexane/EtOAc $=3 / 2)$ afforded compound $39(638 \mathrm{mg}, 79 \%)$ as white amorphous: $[\alpha]^{25} \mathrm{D}+4.5^{\circ}(c$ 0.78, $\left.\mathrm{CHCl}_{3}\right) ;{ }^{1} \mathrm{H}$ NMR $\left(500 \mathrm{MHz}, \mathrm{CDCl}_{3}\right) \delta 8.38(2 \mathrm{H}, \mathrm{d}, J=9.0 \mathrm{~Hz}), 8.09(2 \mathrm{H}, \mathrm{d}, J=9.0 \mathrm{~Hz}), 7.48-7.34(6 \mathrm{H}, \mathrm{m})$, $5.89(1 \mathrm{H}, \mathrm{d}, J=8.0 \mathrm{~Hz}), 5.80(1 \mathrm{H}, \mathrm{d}, J=7.0 \mathrm{~Hz}), 5.56-5.52(2 \mathrm{H}, \mathrm{m}), 5.36(1 \mathrm{H}, \mathrm{d}, J=6.5 \mathrm{~Hz}), 4.79-4.75(3 \mathrm{H}, \mathrm{m})$, 4.21-4.15 (3H, m), $4.04(1 \mathrm{H}, \mathrm{dd}, J=6.5,1.0 \mathrm{~Hz}), 3.61(3 \mathrm{H}, \mathrm{s}), 0.98(9 \mathrm{H}, \mathrm{s}), 0.81(9 \mathrm{H}, \mathrm{s}), 0.17-0.07(12 \mathrm{H}, \mathrm{m}) ;{ }^{13} \mathrm{C}$ NMR (125 MHz, $\left.\mathrm{CDCl}_{3}\right) \delta 169.8,162.2,151.2,150.1,145.4,142.7,137.5,128.44,128.36,127.8,127.7,124.3,102.3$, $96.4,85.6,73.7,72.3,71.6,71.3,70.1,58.6,53.0,25.8,25.7,18.0,17.9,-4.59,-4.65,-5.1$; IR (ATR, $\left.\mathrm{cm}^{-1}\right) 3306$, 2955, 2859, 1666, 1532, 1352, 1251, 840; HRMS (ESI) calcd for $\mathrm{C}_{38} \mathrm{H}_{57} \mathrm{~N}_{4} \mathrm{O}_{13} \mathrm{Si}_{2} \mathrm{~S}[\mathrm{M}+\mathrm{H}]^{+}$863.3030; found $\mathrm{m} / \mathrm{z}$ 863.3032.

\section{Introduction of a fatty-acid side chain using model 1,4-diazepanone}

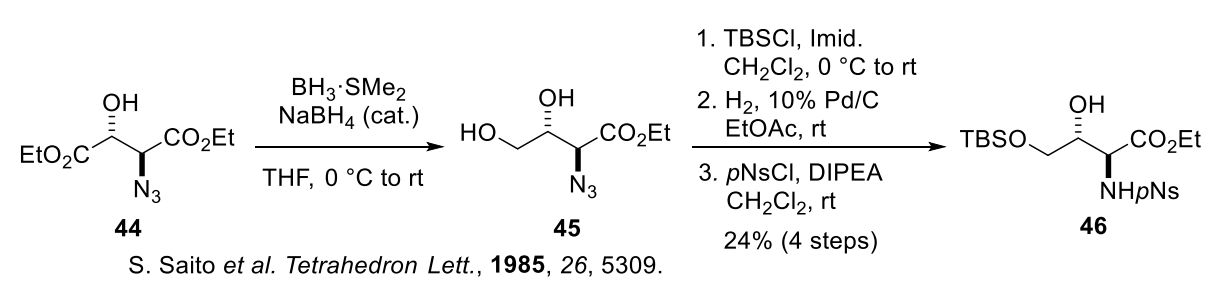

Compound 46: To a solution of $\mathbf{4 4}^{6}(9.94 \mathrm{~g}, 43.0 \mathrm{mmol})$ in THF $(120 \mathrm{ml})$ was added $2.0 \mathrm{M}$ solution of $\mathrm{BH}_{3} \cdot \mathrm{Me}_{2} \mathrm{~S}$ in THF $(25.0 \mathrm{ml}, 50.0 \mathrm{mmol})$ at $0{ }^{\circ} \mathrm{C}$, and the resultant solution was stirred at $0{ }^{\circ} \mathrm{C}$ for $1 \mathrm{~h}$. A catalytic amount of $\mathrm{NaBH}_{4}$ ( $95.8 \mathrm{mg}, 2.53 \mathrm{mmol}$ ) was added to the reaction mixture at $0{ }^{\circ} \mathrm{C}$, and the resultant mixture was stirred at $25^{\circ} \mathrm{C}$ for $1 \mathrm{~h}$. The reaction was quenched with water. After extraction with EtOAc, the combined organic layers were washed with brine and dried over $\mathrm{Na}_{2} \mathrm{SO}_{4}$. Filtration and concentration under reduced pressure afforded a crude product 45 (6.09 g) which was used for the next reaction without further purification. ${ }^{7}$

To a solution of the above crude $45(6.09 \mathrm{~g})$ in $\mathrm{CH}_{2} \mathrm{Cl}_{2}(100 \mathrm{ml})$ were added imidazole $(4.50 \mathrm{~g}, 66.1 \mathrm{mmol})$ and TBSCl $(4.91 \mathrm{~g}, 32.6 \mathrm{mmol})$ at $0{ }^{\circ} \mathrm{C}$, and the resultant solution was stirred at $0{ }^{\circ} \mathrm{C}$ for $1 \mathrm{~h}$. The reaction was then quenched with $1 \mathrm{M}$ aqueous $\mathrm{HCl}$. After extraction with $\mathrm{CHCl}_{3}$, the combined organic layers were washed with brine and dried over $\mathrm{Na}_{2} \mathrm{SO}_{4}$. Filtration and concentration under reduced pressure afforded a crude product $(6.54 \mathrm{~g})$, which was used for the next reaction without further purification.

To a solution of the crude product $(6.54 \mathrm{~g})$ in EtOAc $(70 \mathrm{ml})$ was added $10 \% \mathrm{Pd} / \mathrm{C}$ (2.38 g, $2.24 \mathrm{mmol})$ in EtOAc (30 $\mathrm{ml})$ at $25{ }^{\circ} \mathrm{C}$, and the resultant mixture was stirred at $25^{\circ} \mathrm{C}$ for $8 \mathrm{~h}$. The reaction was filtered through celite and then concentration under reduced pressure afforded a crude product.

To a solution of the above crude product in $\mathrm{CH}_{2} \mathrm{Cl}_{2}(64 \mathrm{ml})$ were added DIPEA $(5.00 \mathrm{ml}, 29.0 \mathrm{mmol})$ and $p \mathrm{NsCl}$ $(5.07 \mathrm{~g}, 22.9 \mathrm{mmol})$ at $0{ }^{\circ} \mathrm{C}$. The resultant solution was stirred at $25{ }^{\circ} \mathrm{C}$ for $0.5 \mathrm{~h}$. The reaction was then quenched with $1 \mathrm{M}$ aqueous $\mathrm{HCl}$. After extraction with $\mathrm{CHCl}_{3}$, the combined organic layers were washed with brine and dried over $\mathrm{Na}_{2} \mathrm{SO}_{4}$. After filtration and concentration under reduced pressure, purification by silica gel column chromatography (hexane/EtOAc $=3: 1)$ afforded compound $46\left(4.68 \mathrm{~g}, 24 \%\right.$ for four steps) as a yellow oil: $[\alpha]^{25} \mathrm{D}+32.1^{\circ}(c 1.21$, $\left.\mathrm{CHCl}_{3}\right) ;{ }^{1} \mathrm{H}$ NMR $\left(500 \mathrm{MHz}, \mathrm{CDCl}_{3}\right) \delta 8.34(2 \mathrm{H}, \mathrm{d}, J=8.6 \mathrm{~Hz}), 8.04(2 \mathrm{H}, \mathrm{d}, J=8.6 \mathrm{~Hz}), 5.90(1 \mathrm{H}, \mathrm{d}, J=9.2 \mathrm{~Hz})$, $4.16(1 \mathrm{H}, \mathrm{dd}, J=9.2,4.6 \mathrm{~Hz}), 4.02-3.93(3 \mathrm{H}, \mathrm{m}), 3.71(2 \mathrm{H}, \mathrm{d}, J=4.6 \mathrm{~Hz}), 2.77(1 \mathrm{H}, \mathrm{d}, J=7.4 \mathrm{~Hz}), 1.14(3 \mathrm{H}, \mathrm{t}, J=7.2$ $\mathrm{Hz}), 0.90(9 \mathrm{H}, \mathrm{s}), 0.08$ (6H, s); ${ }^{13} \mathrm{C}$ NMR (126 MHz, $\left.\mathrm{CDCl}_{3}\right) \delta$ 169.1, 150.0, 145.6, 128.5, 124.2, 71.8, 63.4, 62.0, 58.4, 25.7, 18.1, 13.9, -5.7; IR (ATR, $\left.\mathrm{cm}^{-1}\right)$ 3523, 3267, 1739, 1532, 1349, 1168, 1092; HRMS (ESI) calcd for 


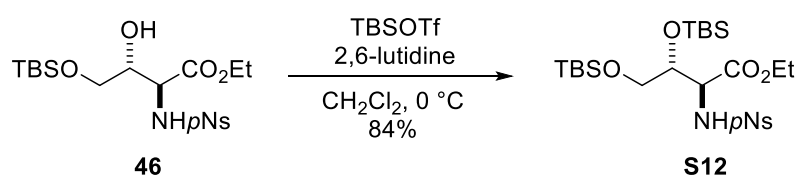

Compound S10: To a solution of alcohol $46(4.60 \mathrm{~g}, 9.94 \mathrm{mmol})$ in $\mathrm{CH}_{2} \mathrm{Cl}_{2}(50 \mathrm{ml})$ were added 2,6-lutidine (1.50 ml, $13.0 \mathrm{mmol})$ and TBSOTf $(3.00 \mathrm{ml}, 13.1 \mathrm{mmol})$ at $0{ }^{\circ} \mathrm{C}$, and the resultant solution was stirred at $0{ }^{\circ} \mathrm{C}$ for $2 \mathrm{~h}$. The reaction was then quenched with $1 \mathrm{M}$ aqueous $\mathrm{HCl}$. After extraction with $\mathrm{CHCl}_{3}$, the combined organic layers were washed with brine and dried over $\mathrm{Na}_{2} \mathrm{SO}_{4}$. After filtration and concentration under reduced pressure, purification by silica gel column chromatography (hexane/EtOAc $=10: 1)$ afforded $\mathbf{S 1 2}(4.81 \mathrm{~g}, 84 \%)$ as a yellow oil: $[\alpha]^{25} \mathrm{D}+35.2^{\circ}(c$ 1.40, $\left.\mathrm{CHCl}_{3}\right) ;{ }^{1} \mathrm{H} \mathrm{NMR}\left(500 \mathrm{MHz}, \mathrm{CDCl}_{3}\right) \delta 8.32(2 \mathrm{H}, \mathrm{d}, J=8.6 \mathrm{~Hz}), 8.03(2 \mathrm{H}, \mathrm{d}, J=8.6 \mathrm{~Hz}), 5.76(1 \mathrm{H}, \mathrm{d}, J=8.0 \mathrm{~Hz})$, $4.26(1 \mathrm{H}, \mathrm{dd}, J=8.0,2.9 \mathrm{~Hz}), 4.09-4.00(3 \mathrm{H}, \mathrm{m}), 3.62(1 \mathrm{H}, \mathrm{dd}, J=10.0,8.3 \mathrm{~Hz}), 3.55(1 \mathrm{H}, \mathrm{dd}, J=10.0,5.1 \mathrm{~Hz}), 1.17$ $(3 \mathrm{H}, \mathrm{t}, J=7.2 \mathrm{~Hz}), 0.91(9 \mathrm{H}, \mathrm{s}), 0.84(9 \mathrm{H}, \mathrm{s}), 0.08-0.05(12 \mathrm{H}, \mathrm{m}) ;{ }^{13} \mathrm{C} \mathrm{NMR}\left(126 \mathrm{MHz}, \mathrm{CDCl}_{3}\right) \delta 168.7,145.0,146.1$, 128.4, 124.1, 74.7, 63.9, 61.9, 58.7, 31.6, 25.7, 22.6, 18.2,17.9, 14.1, 14.0, -4.6, -4.9, -5.5, -5.6; IR (ATR, cm $\left.{ }^{-1}\right) 3293$, 1742, 1533, 1349, 1257, 1171; HRMS (ESI) calcd for $\mathrm{C}_{24} \mathrm{H}_{44} \mathrm{~N}_{2} \mathrm{O}_{8} \mathrm{Si}_{2} \mathrm{SNa}[\mathrm{M}+\mathrm{Na}]^{+}$599.2249; found $m / z$ 599.2232.

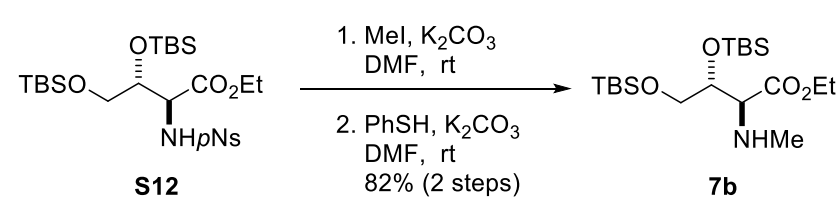

Compound 7b: To a solution of $\mathbf{S 1 2}(1.43 \mathrm{~g}, 2.48 \mathrm{mmol})$ in DMF $(10 \mathrm{~mL})$ were added $\mathrm{K}_{2} \mathrm{CO}_{3}(514 \mathrm{mg}, 3.72 \mathrm{mmol})$ and $\mathrm{MeI}(0.310 \mathrm{ml}, 4.97 \mathrm{mmol})$ at $25{ }^{\circ} \mathrm{C}$, and the resultant solution was stirred at $25^{\circ} \mathrm{C}$ for $2 \mathrm{~h}$. The reaction was then quenched with saturated aqueous $\mathrm{NH}_{4} \mathrm{Cl}$. After extraction with $\mathrm{Et}_{2} \mathrm{O}$, the combined organic layers were washed with brine and dried over $\mathrm{Na}_{2} \mathrm{SO}_{4}$. Filtration and concentrated under reduced pressure afforded the crude product (1.54 g), which was used for further purification.

To a solution of the above crude $(1.54 \mathrm{~g})$ in DMF $(10 \mathrm{~mL})$ were added $\mathrm{K}_{2} \mathrm{CO}_{3}(512 \mathrm{mg}, 3.70 \mathrm{mmol})$ and $\mathrm{PhSH}(0.380$ $\mathrm{ml}, 3.71 \mathrm{mmol}$ ) at $25{ }^{\circ} \mathrm{C}$ and the resultant solution was stirred at $25^{\circ} \mathrm{C}$ for $1.5 \mathrm{~h}$. The reaction was then quenched with water. After extraction with $\mathrm{Et}_{2} \mathrm{O}$, the combined organic layers were washed with brine and dried over $\mathrm{Na}_{2} \mathrm{SO}_{4}$. After filtration and concentrated under reduced pressure, purification by silica gel column chromatography (hexane/EtOAc $=$ 1:1) afforded compound $7 \mathbf{b}\left(0.827 \mathrm{~g}, 82 \%\right.$ for two steps) as a colorless oil: $[\alpha]^{19} \mathrm{D}+13.4^{\circ}\left(c 0.93, \mathrm{CHCl}_{3}\right) ;{ }^{1} \mathrm{H}$ NMR $\left(500 \mathrm{MHz}, \mathrm{CDCl}_{3}\right) \delta 4.24-4.12(\mathrm{~m}, 2 \mathrm{H}), 3.95-3.89(\mathrm{~m}, 1 \mathrm{H}), 3.68(\mathrm{dd}, J=10.2,6.1 \mathrm{~Hz}, 1 \mathrm{H}), 3.57$ (dd, $J=10.2,5.8 \mathrm{~Hz}$, $1 \mathrm{H}), 3.30(\mathrm{~d}, J=4.0 \mathrm{~Hz}, 1 \mathrm{H}), 2.39$ (s, 3H), $1.28(\mathrm{t}, J=7.1 \mathrm{~Hz}, 3 \mathrm{H}), 0.89(\mathrm{~s}, 9 \mathrm{H}), 0.86(\mathrm{~s}, 9 \mathrm{H}), 0.14-0.02(\mathrm{~m}, 12 \mathrm{H}) ;{ }^{13} \mathrm{C}$ NMR (126 MHz, $\left.\mathrm{CDCl}_{3}\right) \delta 172.5,75.4,66.1,64.8,60.4,35.4,25.9,25.7,18.3,18.0,14.3,-4.3,-5.0,-5.4,-5.5$; HRMS (ESI) calcd for $\mathrm{C}_{19} \mathrm{H}_{44} \mathrm{NO}_{4} \mathrm{Si}_{2}[\mathrm{M}+\mathrm{H}]^{+} 406.2809$; found $m / z 406.2813$. 


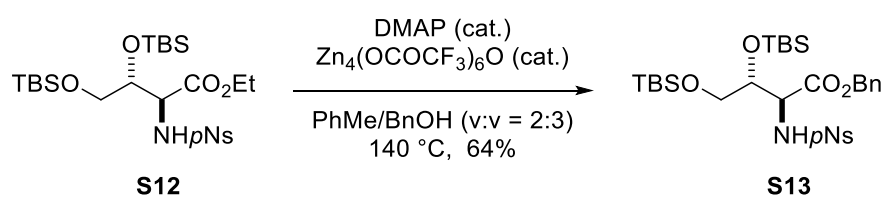

Compound S13: To a solution of $\mathbf{S 1 2}(6.24 \mathrm{~g}, 10.8 \mathrm{mmol})$ in toluene-BnOH $(100 \mathrm{ml}, \mathrm{v} / \mathrm{v}=2: 3)$ were added DMAP (174 mg, $1.42 \mathrm{mmol})$ and $\mathrm{Zn}_{4}\left(\mathrm{OCOCF}_{3}\right)_{6} \mathrm{O}(2.40 \mathrm{~g}, 2.51 \mathrm{mmol})$ at $25{ }^{\circ} \mathrm{C}$, and the resultant solution was stirred at $140{ }^{\circ} \mathrm{C}$ for 3 days. After concentration under reduced pressure at $90{ }^{\circ} \mathrm{C}$, purification by silica gel column chromatography (hexane/EtOAc $=10: 1)$ afforded compound $\mathbf{S 1 3}(4.42 \mathrm{~g}, 64 \%)$ as a yellow oil: $[\alpha]^{25} \mathrm{D}+36.4^{\circ}(c 1.28$, $\left.\mathrm{CHCl}_{3}\right) ;{ }^{1} \mathrm{H} \mathrm{NMR}\left(500 \mathrm{MHz}, \mathrm{CDCl}_{3}\right) \delta 8.18(2 \mathrm{H}, \mathrm{d}, J=8.6 \mathrm{~Hz}), 7.94(2 \mathrm{H}, \mathrm{d}, J=8.6 \mathrm{~Hz}), 7.39-7.27(3 \mathrm{H}, \mathrm{m}), 7.21-7.18$ $(2 \mathrm{H}, \mathrm{m}), 5.78(1 \mathrm{H}, \mathrm{d}, J=8.0 \mathrm{~Hz}), 5.00(2 \mathrm{H}, \mathrm{d}, J=3.4 \mathrm{~Hz}), 4.31(1 \mathrm{H}, \mathrm{dd}, J=8.3,2.0 \mathrm{~Hz}), 4.10-4.03(1 \mathrm{H}, \mathrm{m}), 3.59(1 \mathrm{H}$, $\mathrm{dd}, J=10.0,8.3 \mathrm{~Hz}), 3.53(1 \mathrm{H}, \mathrm{dd}, J=10.0,4.9 \mathrm{~Hz}), 0.89(9 \mathrm{H}, \mathrm{s}), 0.84(9 \mathrm{H}, \mathrm{s}), 0.10-0.01(12 \mathrm{H}, \mathrm{m}) ;{ }^{13} \mathrm{C}$ NMR $(126$ $\left.\mathrm{MHz}, \mathrm{CDCl}_{3}\right) \delta 168.6,149.9,145.9,134.6,128.7,128.5,128.4,128.3,124.1,74.8,67.6,64.0,58.8,31.6,25.8,25.6$, 22.7, 18.2, 17.9, 14.1, -4.6, -4.9, -5.5, -5.6; IR (ATR, $\left.\mathrm{cm}^{-1}\right)$ 3288, 1742, 1532, 1349, 1257, 1170; HRMS (ESI) calcd for $\mathrm{C}_{29} \mathrm{H}_{46} \mathrm{~N}_{2} \mathrm{O}_{8} \mathrm{Si}_{2} \mathrm{SNa}[\mathrm{M}+\mathrm{Na}]^{+}$661.2406; found $m / z 661.2422$.

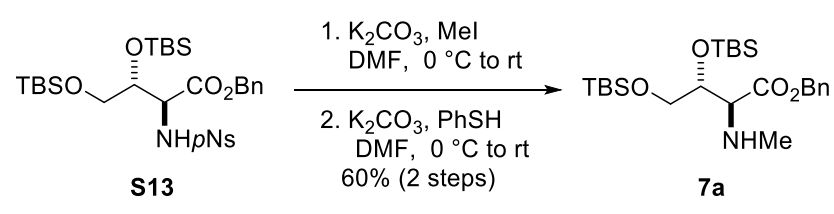

Compound 7a: To a solution of $\mathbf{S 1 3}(4.34 \mathrm{~g}, 6.79 \mathrm{mmol})$ in DMF $(30 \mathrm{ml})$ were added $\mathrm{K}_{2} \mathrm{CO}_{3}(1.13 \mathrm{~g}, 8.18 \mathrm{mmol})$ and MeI $(2.00 \mathrm{ml}, 32.1 \mathrm{mmol})$ at $0{ }^{\circ} \mathrm{C}$ and the resultant solution was stirred at $25{ }^{\circ} \mathrm{C}$ for $4 \mathrm{~h}$. The reaction was then quenched with saturated aqueous $\mathrm{NH}_{4} \mathrm{Cl}$. After extraction with $\mathrm{Et}_{2} \mathrm{O}$, the combined organic layers were washed with brine and dried over $\mathrm{Na}_{2} \mathrm{SO}_{4}$. Filtration and concentrated under reduced pressure afforded the crude product $(4.41 \mathrm{~g})$, which was used for further purification.

To a solution of the above crude $(4.41 \mathrm{~g})$ in DMF $(34 \mathrm{ml})$ were added $\mathrm{K}_{2} \mathrm{CO}_{3}(1.15 \mathrm{~g}, 8.32 \mathrm{mmol})$ and $\mathrm{PhSH}(0.84 \mathrm{ml}$, $8.21 \mathrm{mmol}$ ) at $0{ }^{\circ} \mathrm{C}$ and the resultant solution was stirred at $25^{\circ} \mathrm{C}$ for $2.5 \mathrm{~h}$. The reaction was then quenched with water. After extraction with $\mathrm{Et}_{2} \mathrm{O}$, the combined organic layers were washed with brine and dried over $\mathrm{Na}_{2} \mathrm{SO}_{4}$. After filtration and concentrated under reduced pressure, purification by silica gel column chromatography (hexane/EtOAc $=$ 20:1) afforded compound $7 \mathbf{a}\left(1.90 \mathrm{~g}, 60 \%\right.$ for two steps) as a yellow oil: $[\alpha]^{25} \mathrm{D}+12.0^{\circ}\left(c 1.33, \mathrm{CHCl}_{3}\right) ;{ }^{1} \mathrm{H} \mathrm{NMR}(500$ $\left.\mathrm{MHz} \mathrm{CDCl}_{3}\right) \delta$ 7.38-7.28 (5H, m), $5.19(1 \mathrm{H}, \mathrm{d}, J=12.6 \mathrm{~Hz}), 5.13(1 \mathrm{H}, \mathrm{d}, J=12.6 \mathrm{~Hz}), 3.98-3.90(1 \mathrm{H}, \mathrm{m}), 3.64(1 \mathrm{H}$, dd, $J=10.3,6.3 \mathrm{~Hz}), 3.54(1 \mathrm{H}, \mathrm{dd}, J=10.3,5.7 \mathrm{~Hz}), 3.36(1 \mathrm{H}, \mathrm{d}, J=3.4 \mathrm{~Hz}), 2.38(3 \mathrm{H}, \mathrm{s}), 0.88-0.83(18 \mathrm{H}, \mathrm{m}), 0.07$ $(6 \mathrm{H}, \mathrm{s}), 0.05(6 \mathrm{H}, \mathrm{s}) . ;{ }^{13} \mathrm{C} \mathrm{NMR}\left(126 \mathrm{MHz}, \mathrm{CDCl}_{3}\right) \delta 172.3,135.9,128.5,128.3,128.1,75.6,66.3,66.0,64.7,35.4$, 25.9, 25.8, 18.3, 18.0, -4.3, -5.0, -5.4, -5.5; IR (ATR, $\left.\mathrm{cm}^{-1}\right)$ 2929, 1739, 1463, 1253, 1109, 834; HRMS (ESI) calcd for $\mathrm{C}_{24} \mathrm{H}_{46} \mathrm{NO}_{4} \mathrm{Si}_{2}[\mathrm{M}+\mathrm{H}]^{+} 468.2960$; found $m / z 468.2954$. 


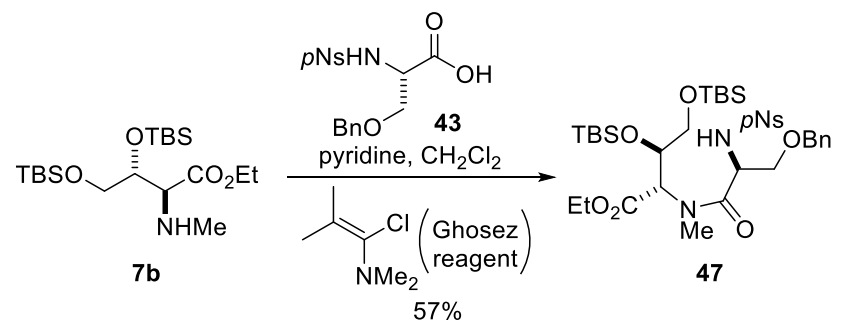

Compound 47: To a solution of carboxylic acid $\mathbf{4 3}^{8}(889 \mathrm{mg}, 2.34 \mathrm{mmol})$ in $\mathrm{CH}_{2} \mathrm{Cl}_{2}(6.0 \mathrm{~mL})$ was added Ghosez

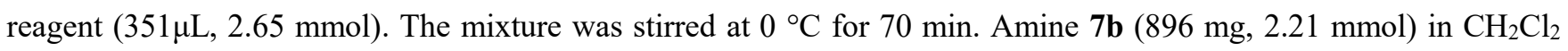
$(10.3 \mathrm{~mL})$ and pyridine $(250 \mu \mathrm{L}, 3.11 \mathrm{mmol})$ were then added to the reaction mixture. The resultant solution was stirred at $0{ }^{\circ} \mathrm{C}$ for $15 \mathrm{~min}$. After addition of water to the reaction, the mixture was extracted with $\mathrm{CHCl}_{3}$. The combined organic layers were washed with brine and dried over $\mathrm{Na}_{2} \mathrm{SO}_{4}$. After filtration and concentrated under reduced pressure, purification of the residue by silica gel column chromatography (hexane/EtOAc $=10: 1)$ afforded $47(963 \mathrm{mg}, 57 \%)$ as a yellow oil: $[\alpha]^{18} \mathrm{D}+18.1^{\circ}\left(c 1.05, \mathrm{CHCl}_{3}\right) ;{ }^{1} \mathrm{H} \mathrm{NMR}\left(500 \mathrm{MHz}, \mathrm{CDCl}_{3}\right) \delta 8.17(2 \mathrm{H}, \mathrm{d}, J=8.3 \mathrm{~Hz}), 7.98(2 \mathrm{H}, \mathrm{d}, J=$ $8.3 \mathrm{~Hz}), 7.29-7.25(4 \mathrm{H}, \mathrm{m}), 7.16(2 \mathrm{H}, \mathrm{d}, J=6.9 \mathrm{~Hz}), 5.83-5.81(1 \mathrm{H}, \mathrm{m}), 5.29-5.27(1 \mathrm{H}, \mathrm{m}), 4.61(1 \mathrm{H}, \mathrm{dd}, J=5.7,2.9$ $\mathrm{Hz}), 4.42(1 \mathrm{H}, \mathrm{d}, J=11.7 \mathrm{~Hz}), 4.33(1 \mathrm{H}, \mathrm{d}, J=11.7 \mathrm{~Hz}), 4.16-4.09(2 \mathrm{H}, \mathrm{m}), 3.97-3.95(1 \mathrm{H}, \mathrm{m}), 3.74(1 \mathrm{H}, \mathrm{dd}, J=8.6$, $4.3 \mathrm{~Hz}), 3.61-3.58(2 \mathrm{H}, \mathrm{m}), 3.49(1 \mathrm{H}, \mathrm{s}), 3.48(1 \mathrm{H}, \mathrm{dd}, J=8.3,4.2 \mathrm{~Hz}), 3.14(3 \mathrm{H}, \mathrm{s}), 3.04-2.93(1 \mathrm{H}, \mathrm{m}), 1.21(3 \mathrm{H}, \mathrm{t}, J$ $=7.0 \mathrm{~Hz}), 0.97-0.81(18 \mathrm{H}, \mathrm{m}), 0.11-0.07(12 \mathrm{H}, \mathrm{m}) ;{ }^{13} \mathrm{C} \mathrm{NMR}\left(126 \mathrm{MHz}, \mathrm{CDCl}_{3}\right) \delta 150.3,128.5,128.3,127.7,124.0$, 74.2, 73.7, 70.9, 60.9, 53.9, 26.0, 25.8, 19.3, 17.9, 14.2; IR (ATR, $\mathrm{cm}^{-1}$ ) 2995, 2930, 1739, 1647, 1536, 1473, 1349, 1254, 1169, 1110; HRMS (ESI) calcd for $\mathrm{C}_{35} \mathrm{H}_{57} \mathrm{~N}_{3} \mathrm{O}_{10} \mathrm{Si}_{2} \mathrm{~S}$ [M-H] $]^{-}$766.3263; found: $m / z 766.3230$.

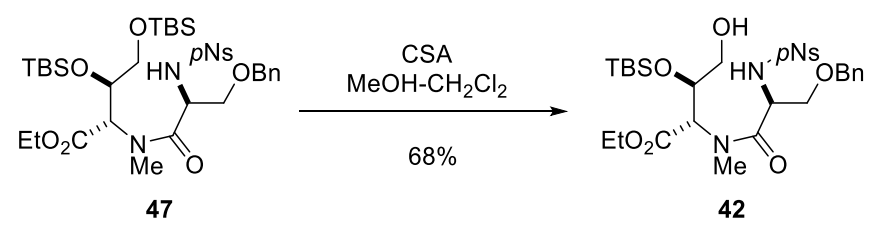

Compound 42: To a solution of $47(941 \mathrm{mg}, 1.23 \mathrm{mmol})$ in $\mathrm{MeOH}-\mathrm{CH}_{2} \mathrm{Cl}_{2}(10 \mathrm{~mL}, \mathrm{v} / \mathrm{v}=2: 3)$ was added CSA (33.9 $\mathrm{mg}, 146 \mu \mathrm{mol})$ at $0{ }^{\circ} \mathrm{C}$. The reaction mixture was stirred at room temperature for $15 \mathrm{~h}$. The reaction solution was extracted with $\mathrm{CHCl}_{3}$. The combined organic layers were washed with brine and dried over $\mathrm{Na}_{2} \mathrm{SO}_{4}$. After filtration and concentration under reduced pressure, purification of the residue by silica gel column chromatography (hexane/EtOAc $=4: 1)$ afforded $42(548 \mathrm{mg}, 68 \%)$ as a yellow amorphous: $[\alpha]^{18} \mathrm{D}+0.7^{\circ}\left(c 0.67, \mathrm{CHCl}_{3}\right) ;{ }^{1} \mathrm{H} \mathrm{NMR}(500$ $\left.\mathrm{MHz} \mathrm{CDCl}_{3}\right) \delta 8.21(2 \mathrm{H}, \mathrm{d}, J=8.0 \mathrm{~Hz}), 7.99(2 \mathrm{H}, \mathrm{d}, J=8.0 \mathrm{~Hz}), 7.31-7.25(4 \mathrm{H}, \mathrm{m}), 7.17(2 \mathrm{H}, \mathrm{d}, J=6.6 \mathrm{~Hz})$, 6.02-6.00 (1H, m), $4.74(1 \mathrm{H}, \mathrm{d}, J=5.4 \mathrm{~Hz}), 4.58-4.56(1 \mathrm{H}, \mathrm{m}), 4.43(1 \mathrm{H}, \mathrm{d}, J=11.8 \mathrm{~Hz}), 4.38(1 \mathrm{H}, \mathrm{d}, J=11.9 \mathrm{~Hz})$, 4.14-4.10 (3H, m), 3.66-3.50 (3H, m), $3.34(1 \mathrm{H}, \mathrm{d}, J=11.2 \mathrm{~Hz}), 3.10(2 \mathrm{H}, \mathrm{s}), 3.03-2.89(1 \mathrm{H}, \mathrm{m}), 1.26-1.19(3 \mathrm{H}, \mathrm{m})$, 0.88 (9H, s), 0.12-0.05 (6H, m); ${ }^{13} \mathrm{C}$ NMR $\left(126 \mathrm{MHz}, \mathrm{CDCl}_{3}\right) \delta 168.6,150.3,146.6,137.0,128.5,128.2,128.1,127.6$, 124.2, 73.6, 71.4, 63.6, 61.4, 53.4, 34.4, 25.7, 19.2, 18.0, 14.1; IR (ATR, $\mathrm{cm}^{-1}$ ) 2946, 2930, 2858, 1742, 1645, 1532, 1350; HRMS (ESI) calcd for $\mathrm{C}_{29} \mathrm{H}_{43} \mathrm{~N}_{3} \mathrm{O}_{10} \mathrm{SiS}[\mathrm{M}+\mathrm{H}]^{+} 654.2490$; found: $m / z 654.2511$. 


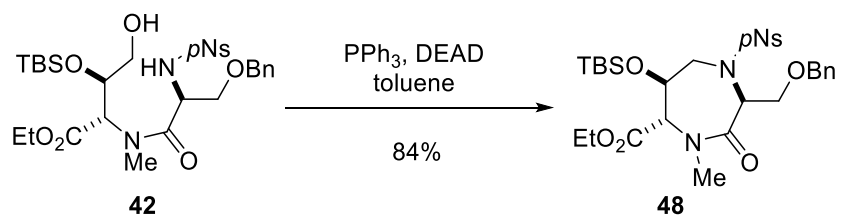

Compound 48: To a solution of compound $42(618 \mathrm{mg}, 945 \mu \mathrm{mol})$ in toluene $(19 \mathrm{~mL})$ were added $\mathrm{PPh}_{3}(273 \mathrm{mg}, 1.04$ mmol), DEAD $(475 \mu \mathrm{L}, 1.04 \mathrm{mmol})$ at $0{ }^{\circ} \mathrm{C}$. The reaction solution was stirred at $0{ }^{\circ} \mathrm{C}$ for $0.5 \mathrm{~h}$. After concentration under reduced pressure, the mixture was purified by silica gel column chromatography (hexane/EtOAc $=4: 1$ ) afforded $48(503 \mathrm{mg}, 84 \%)$ as a white solid: ${ }^{1} \mathrm{H}$ NMR $\left(500 \mathrm{MHz}, \mathrm{CDCl}_{3}\right) \delta 8.03(2 \mathrm{H}, \mathrm{d}, J=8.9 \mathrm{~Hz}), 7.85(2 \mathrm{H}, \mathrm{d}, J=8.9 \mathrm{~Hz})$, 7.22-7.21 (3H, m), 6.92-6.91 (2H, m), 5.00-4.99 (1H, m), 4.89-4.87 (1H, m), 4.35-4.34 (2H, m), 4.22-4.14 (3H, m), $4.16(3 \mathrm{H}, \mathrm{s}), 4.04(1 \mathrm{H}, \mathrm{d}, J=2.9 \mathrm{~Hz}), 4.01-3.92(2 \mathrm{H}, \mathrm{m}), 3.55(1 \mathrm{H}, \mathrm{d}, J=3.7 \mathrm{~Hz}), 3.53(1 \mathrm{H}, \mathrm{d}, J=3.7 \mathrm{~Hz}), 3.19(4 \mathrm{H}$, d, $J=6.3 \mathrm{~Hz}), 3.17-3.15(4 \mathrm{H}, \mathrm{m}), 1.36(3 \mathrm{H}, \mathrm{t}, J=7.2 \mathrm{~Hz}), 0.90(9 \mathrm{H}, \mathrm{s}), 0.16-0.13(6 \mathrm{H}, \mathrm{m}) ;{ }^{13} \mathrm{C}$ NMR $(126 \mathrm{MHz}$, $\left.\mathrm{CDCl}_{3}\right) \delta 168.6,153.8,137.2,128.4,128.3,127.9,127.4,123.8,73.2,72.2,62.7,56.7,48.8,40.5,25.6,17.9,13.9$; IR (ATR, $\mathrm{cm}^{-1}$ ) 2932, 2858, 1743, 1651, 1535, 1476, 1350, 1253; HRMS (ESI) calcd for $\mathrm{C}_{29} \mathrm{H}_{41} \mathrm{~N}_{3} \mathrm{O}_{9} \mathrm{SiS}[\mathrm{M}-\mathrm{H}]^{-}$ 634.2238; found: $m / z 634.2260$.

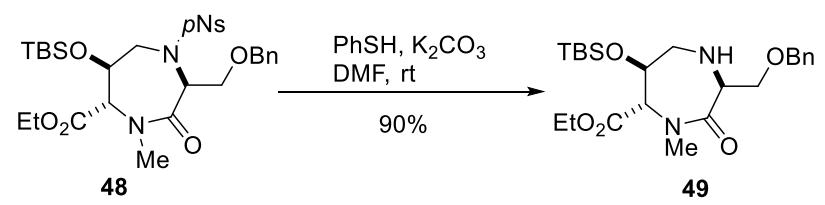

Compound 49: To a solution of 48 (498 mg, $783 \mu \mathrm{mol})$ in DMF $(8.0 \mathrm{~mL})$ were added PhSH $(270 \mu \mathrm{L}, 2.64 \mathrm{mmol})$ and $\mathrm{K}_{2} \mathrm{CO}_{3}(143 \mathrm{mg}, 1.03 \mathrm{mmol})$ at $0{ }^{\circ} \mathrm{C}$. The reaction solution was stirred at $\mathrm{rt}$ for $20 \mathrm{~h}$. The reaction was quenched with saturated aqueous $\mathrm{NH}_{4} \mathrm{Cl}$. The mixture was extracted with $\mathrm{Et}_{2} \mathrm{O}$. The combined organic layers were washed with brine and dried over $\mathrm{Na}_{2} \mathrm{SO}_{4}$. After filtration and concentration under reduced pressure, purification of the residue by silica gel column chromatography (hexane/EtOAc $=2: 1)$ afforded $49(319 \mathrm{mg}, 90 \%)$ as a yellow oil: $[\alpha]^{19} \mathrm{D}-6.6^{\circ}(c 1.04$, $\left.\mathrm{CHCl}_{3}\right) ;{ }^{1} \mathrm{H}$ NMR (500 MHz, $\left.\mathrm{CDCl}_{3}\right) \delta$ 7.33-7.25 (5H, m), $4.57(2 \mathrm{H}, \mathrm{d}, J=4.9 \mathrm{~Hz}), 4.51(1 \mathrm{H}, \mathrm{s}), 4.26-4.20(2 \mathrm{H}, \mathrm{m})$, $3.95(1 \mathrm{H}, \mathrm{d}, J=4.3 \mathrm{~Hz}), 3.91(1 \mathrm{H}, \mathrm{d}, J=4.3 \mathrm{~Hz}), 3.89(1 \mathrm{H}, \mathrm{d}, J=4.3 \mathrm{~Hz}), 3.66(1 \mathrm{H}, \mathrm{t}, J=9.3 \mathrm{~Hz}), 3.46(1 \mathrm{H}, \mathrm{s}), 3.07$ $(3 \mathrm{H}, \mathrm{s}), 2.98(1 \mathrm{H}, \mathrm{d}, J=4.0 \mathrm{~Hz}), 2.95(1 \mathrm{H}, \mathrm{d}, J=4.0 \mathrm{~Hz}), 2.88(1 \mathrm{H}, \mathrm{s}), 1.28(3 \mathrm{H}, \mathrm{t}, J=7.2 \mathrm{~Hz}), 0.88(9 \mathrm{H}, \mathrm{s}), 0.10-0.08$ $(6 \mathrm{H}, \mathrm{m}) ;{ }^{13} \mathrm{C}$ NMR $\left(126 \mathrm{MHz}, \mathrm{CDCl}_{3}\right) \delta 168.8,138.4,128.3,127.7,127.5,73.3,68.5,61.8,61.3,39.4,25.7,17.9$, 14.2; IR (ATR, $\mathrm{cm}^{-1}$ ) 2936, 2858, 1739, 1647, 1465, 1395; HRMS (ESI) calcd for $\mathrm{C}_{23} \mathrm{H}_{38} \mathrm{~N}_{2} \mathrm{O}_{5} \mathrm{Si}[\mathrm{M}+\mathrm{H}]^{+} 451.2582$; found: $m / z 451.2623$.

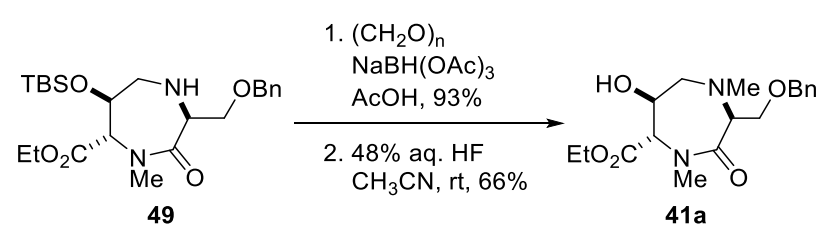

Compound 41a: To a solution of $49(31.7 \mathrm{mg}, 70.3 \mu \mathrm{mol})$ in $\mathrm{CH}_{2} \mathrm{Cl}_{2}(2.5 \mathrm{~mL})$ were added $\left(\mathrm{CH}_{2} \mathrm{O}\right)_{\mathrm{n}}(35.5 \mathrm{mg}, 1.18$ $\mathrm{mmol}), \mathrm{AcOH}(50.0 \mu \mathrm{L}, 0.874 \mathrm{mmol})$ and $\mathrm{NaBH}(\mathrm{OAc})_{3}(67.5 \mathrm{mg}, 318 \mu \mathrm{mol})$ at $\mathrm{rt}$. The reaction mixture was stirred at rt for $10 \mathrm{~h}$. The reaction was quenched with saturated aqueous $\mathrm{NaHCO}_{3}$. The mixture was extracted with $\mathrm{CHCl}_{3}$. The 
combined organic layers were washed with brine and dried over $\mathrm{Na}_{2} \mathrm{SO}_{4}$. After filtration and concentration under reduced pressure, purification of the residue by silica gel column chromatography (hexane/EtOAc $=2: 1$ ) afforded amine $\mathbf{S 1 4}$ (30.3 mg, 93\%) as a yellow oil: ${ }^{1} \mathrm{H}$ NMR (500 MHz, $\left.\mathrm{CDCl}_{3}\right) \delta$ 7.32-7.26 (5H, m), 4.57-4.55 (3H, m), 4.24-4.18 (2H, m), 3.93-3.86 (3H, m), 3.56-3.54 (1H, m), 3.08-3.00 (5H, m), $2.44(3 \mathrm{H}, \mathrm{s}), 1.26(3 \mathrm{H}, \mathrm{t}, J=7.0 \mathrm{~Hz})$, $0.86(9 \mathrm{H}, \mathrm{s}), 0.09(6 \mathrm{H}, \mathrm{s}) ;{ }^{13} \mathrm{C} \mathrm{NMR}\left(126 \mathrm{~Hz}, \mathrm{CDCl}_{3}\right) \delta 138.6,128.3,127.6,127.4,73.2,68.4,67.8,61.5,39.4,25.7$, 17.8, 15.1, 14.3, -4.8, -5.1; IR (ATR, $\mathrm{cm}^{-1}$ ) 2954, 1647, 1395, 1254, 1102; HRMS (ESI) calcd for $\mathrm{C}_{24} \mathrm{H}_{40} \mathrm{~N}_{2} \mathrm{O}_{5} \mathrm{Si}$ $[\mathrm{M}+\mathrm{H}]^{+}$465.2704; found: $m / z 465.2779$

To a solution of the above amine $\mathbf{S 1 4}(30.3 \mathrm{mg}, 65.2 \mu \mathrm{mol})$ in $\mathrm{MeCN}(1.0 \mathrm{~mL})$ was added 48\% aqueous $\mathrm{HF}(1.2 \mathrm{~mL})$ at $0{ }^{\circ} \mathrm{C}$. The reaction mixture was then stirred at $\mathrm{rt}$ for $19 \mathrm{~h}$. The reaction was quenched with saturated aqueous $\mathrm{NaHCO}_{3}$ at $0{ }^{\circ} \mathrm{C}$. The mixture was extracted with $\mathrm{CHCl}_{3}$. The combined organic layers were washed with brine and dried over $\mathrm{Na}_{2} \mathrm{SO}_{4}$. After filtration and concentration under reduced pressure, purification of the residue by silica gel column chromatography (hexane/EtOAc $=1: 1)$ afforded $41 \mathrm{a}(15.2 \mathrm{mg}, 66 \%)$ as a colorless oil: $[\alpha]^{18} \mathrm{D}+28.3^{\circ}(c 0.62$, $\left.\mathrm{CHCl}_{3}\right) ;{ }^{1} \mathrm{H}$ NMR $\left(500 \mathrm{MHz}, \mathrm{CDCl}_{3}\right) \delta$ 7.38-7.27 (m, 5H), 4.77-4.65 (m, 1H), $4.60(\mathrm{~d}, J=11.5 \mathrm{~Hz}, 1 \mathrm{H}), 4.48(\mathrm{~d}, J=$ $11.6 \mathrm{~Hz}, 1 \mathrm{H}), 4.23-4.09(\mathrm{~m}, 2 \mathrm{H}), 4.05(\mathrm{dd}, J=10.1,5.0 \mathrm{~Hz}, 1 \mathrm{H}), 4.01(\mathrm{~d}, J=5.7 \mathrm{~Hz}, 1 \mathrm{H}), 3.94(\mathrm{dd}, J=10.1,1.8 \mathrm{~Hz}$, $1 \mathrm{H}), 3.61-3.58(\mathrm{~m}, 1 \mathrm{H}), 3.15(\mathrm{~s}, 3 \mathrm{H}), 3.01(\mathrm{dd}, J=14.0,8.0 \mathrm{~Hz}, 1 \mathrm{H}), 2.93(\mathrm{dd}, J=14.0,2.2 \mathrm{~Hz}, 1 \mathrm{H}), 2.29(\mathrm{~s}, 3 \mathrm{H})$, $1.25(\mathrm{t}, J=7.1 \mathrm{~Hz}, 3 \mathrm{H}) ;{ }^{13} \mathrm{C} \mathrm{NMR}\left(126 \mathrm{MHz}, \mathrm{CDCl}_{3}\right) \delta 170.8,169.2,136.7,128.5,128.23,128.17,74.3,71.6,68.7$, 67.20, 67.17, 60.9, 55.6, 43.5, 39.8, 14.4; HRMS (FAB) calcd for $\mathrm{C}_{18} \mathrm{H}_{27} \mathrm{~N}_{2} \mathrm{O}_{5}[\mathrm{M}+\mathrm{H}]^{+}$351.1920; found: $\mathrm{m} / z$ 351.1917.

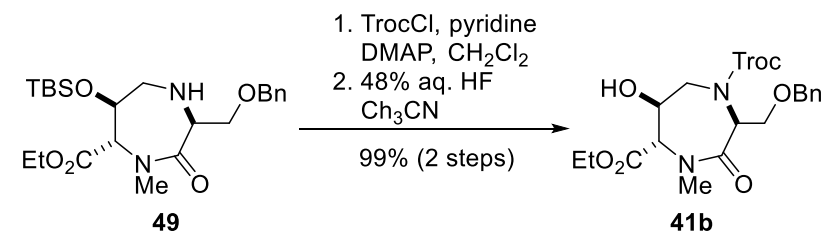

Compound 41b: To a solution of $49(10.6 \mathrm{mg}, 0.0226 \mathrm{mmol})$ in $\mathrm{CH}_{2} \mathrm{Cl}_{2}(1.5 \mathrm{ml})$ were added pyridine $(11.0 \mu \mathrm{L}, 0.136$ mmol), DMAP (5.3 mg, $0.047 \mathrm{mmol})$ and $\mathrm{TrocCl}(20 \mu \mathrm{L}, 0.148 \mathrm{mmol})$ at $0{ }^{\circ} \mathrm{C}$ and the resultant solution was stirred at $0{ }^{\circ} \mathrm{C}$ for $0.5 \mathrm{~h}$. The reaction was then quenched with saturated aqueous $\mathrm{NH}_{4} \mathrm{Cl}$. The mixture was extracted with $\mathrm{CHCl}_{3}$, the organic layers were washed with brine and dried over $\mathrm{Na}_{2} \mathrm{SO}_{4}$. After filtration and concentrated under reduced pressure, the obtained residue was filtered through silica gel column (hexane/EtOAc $=4: 1$ ) afforded the crude product $(16.3 \mathrm{mg})$ as a colorless oil.

To a solution of the above crude product $(16.3 \mathrm{mg})$ in $\mathrm{MeCN}(1.0 \mathrm{~mL})$ was added $48 \%$ aqueous $\mathrm{HF}(0.40 \mathrm{~mL})$ at $\mathrm{rt}$. The reaction mixture was then stirred at $\mathrm{rt}$ for $8.5 \mathrm{~h}$. The reaction was quenched with saturated aqueous $\mathrm{NaHCO}_{3}$. The mixture was extracted with $\mathrm{CHCl}_{3}$. The combined organic layers were washed with brine and dried over $\mathrm{Na}_{2} \mathrm{SO}_{4}$. After filtration and concentration under reduced pressure, purification of the residue by silica gel column chromatography (hexane/EtOAc $=1: 2)$ afforded $\mathbf{4 1 b}\left(12.0 \mathrm{mg}, 99 \%\right.$ for two steps) as a colorless oil: $[\alpha]^{28} \mathrm{D}+73.8^{\circ}\left(c 0.95, \mathrm{CHCl}_{3}\right)$; The mixture of rotamers were observed (see NMR spectrum), ${ }^{1} \mathrm{H}$ NMR $\left(500 \mathrm{MHz}, \mathrm{CDCl}_{3}\right) \delta 7.35-7.21(\mathrm{~m}, 5 \mathrm{H}), 5.06-$ $4.88(\mathrm{~m}, 2 \mathrm{H}), 4.88-4.80(\mathrm{~m}, 5 \mathrm{H}), 4.80-4.69(\mathrm{~m}, 1 \mathrm{H}), 4.63-4.54$ (m, 1H), $4.52-4.39(\mathrm{~m}, 2 \mathrm{H}), 4.38-4.08(\mathrm{~m}, 6 \mathrm{H})$, $3.80(\mathrm{~d}, J=9.7 \mathrm{~Hz}, 1 \mathrm{H}), 3.20(\mathrm{~s}, 4 \mathrm{H}), 2.56-2.18(\mathrm{~m}, 1 \mathrm{H}), 1.27(\mathrm{t}, J=7.2 \mathrm{~Hz}, 3 \mathrm{H}) ;{ }^{13} \mathrm{C} \mathrm{NMR}\left(126 \mathrm{MHz}, \mathrm{CDCl}_{3}\right) \delta$ 169.4, 167.7, 152.9, 137.9, 128.39, 128.36, 128.35, 128.1, 127.72, 127.65, 127.63, 127.57, 124.2, 75.7, 73.5, 73.4, 70.2, 69.4, 62.4, 61.6, 61.2, 40.6, 14.1, 13.9; HRMS (FAB) calcd for $\mathrm{C}_{20} \mathrm{H}_{26} \mathrm{Cl}_{3} \mathrm{~N}_{2} \mathrm{O}_{7}[\mathrm{M}+\mathrm{H}]^{+}$511.0806; found: $\mathrm{m} / z$ 511.0798 . 


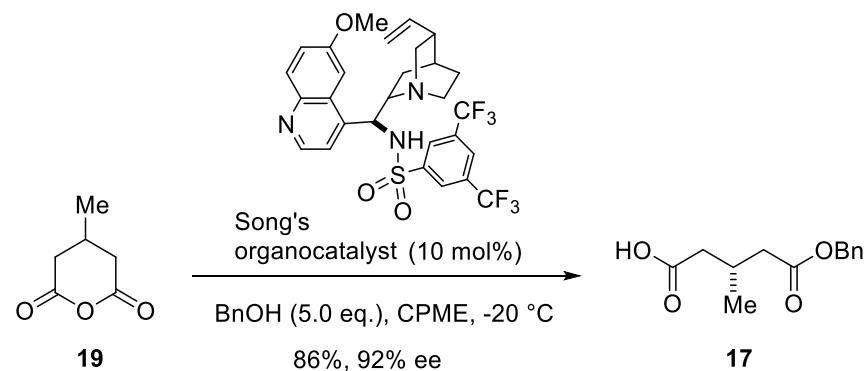

Compound 17: To a solution of $19(64.1 \mathrm{mg}, 0.500 \mathrm{mmol})$ and Song's organocatalyst $(30.0 \mathrm{mg}, 0.050 \mathrm{mmol}) \mathrm{in}$ CPME $(5.0 \mathrm{ml})$ was added $\mathrm{BnOH}(0.26 \mathrm{ml}, 2.51 \mathrm{mmol})$ dropwise at $-20^{\circ} \mathrm{C}$. After the solution was stirred for $4 \mathrm{~h}$, the reaction was quenched with $1 \mathrm{M}$ aqueous $\mathrm{HCl}$. The mixture was extracted with $\mathrm{Et}_{2} \mathrm{O}$. The combined organic layers were washed with saturated aqueous $\mathrm{NaHCO}_{3}$. The water layer was acidified with $1 \mathrm{M}$ aqueous $\mathrm{HCl}$, and extracted with EtOAc three times. The combined organic layers were dried over $\mathrm{MgSO}_{4}$. After filtration and concentration under reduced pressure, purification by silica gel column chromatography (hexane/EtOAc $=2: 1)$ afforded $17(101.0 \mathrm{mg}, 86 \%$, 92\%ee) as a colorless oil: $[\alpha]^{25} \mathrm{D}-3.1^{\circ}\left(c 1.00, \mathrm{CHCl}_{3}\right) ;{ }^{1} \mathrm{H} \mathrm{NMR}\left(500 \mathrm{MHz}, \mathrm{CDCl}_{3}\right) \delta 11.16(1 \mathrm{H}, \mathrm{br} \mathrm{s}), 7.36-7.29(5 \mathrm{H}$, m), $5.12(2 \mathrm{H}, \mathrm{s}), 2.53-2.39(3 \mathrm{H}, \mathrm{m}), 2.36-2.24(2 \mathrm{H}, \mathrm{m}), 1.04(3 \mathrm{H}, \mathrm{d}, J=6.3 \mathrm{~Hz}) ;{ }^{13} \mathrm{C} \mathrm{NMR}\left(126 \mathrm{MHz}, \mathrm{CDCl}_{3}\right) \delta 178.7$, 172.1, 135.7, 128.5, 128.2 (2), 66.2, 40.6, 40.4, 27.1, 19.7; IR (ATR, $\mathrm{cm}^{-1}$ ) 2965, 1733, 1706, 1155, 750; HRMS (ESI) calcd for $\mathrm{C}_{13} \mathrm{H}_{16} \mathrm{O}_{4} \mathrm{Na}[\mathrm{M}+\mathrm{Na}]^{+} 259.0941$; found $m / z 259.0935$.

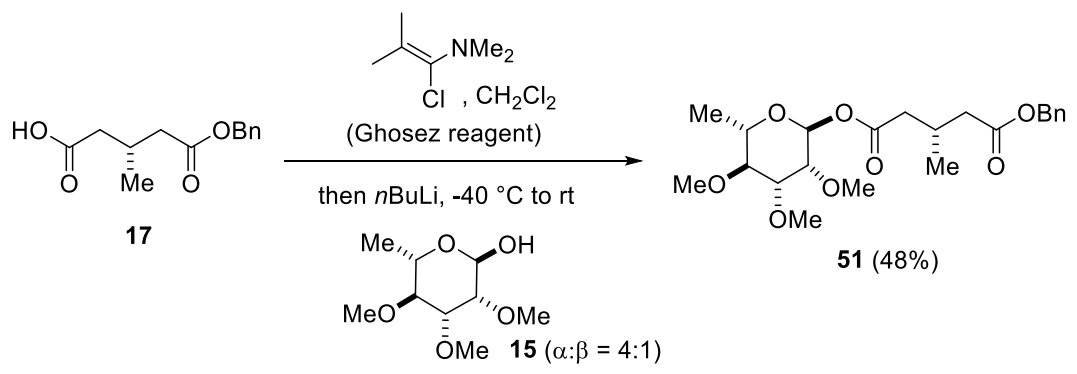

Compound 51: To a solution of $17(60.8 \mathrm{mg}, 0.26 \mathrm{mmol})$ in $\mathrm{CH}_{2} \mathrm{Cl}_{2}(2.0 \mathrm{~mL})$ was added Ghosez reagent $(37.0 \mu \mathrm{L}$, $0.28 \mathrm{mmol}$ ) one portion at $0{ }^{\circ} \mathrm{C}$, and the resultant solution was allowed to warm to room temperature. After the resultant solution was stirred for $30 \mathrm{~min}$, the resulting mixture was concentrated to give a crude acid chloride.

To a solution of $15(53.1 \mathrm{mg}, 0.26 \mathrm{mmol})$ in THF $(2.0 \mathrm{ml})$ were added $n$-BuLi $(0.17 \mathrm{ml}, 1.67 \mathrm{M})$ and the solution of the above acid chloride at $-40{ }^{\circ} \mathrm{C}$. The mixture was stirred at the same temperature for $1 \mathrm{~h}$, and the resultant solution was allowed to warm to $25^{\circ} \mathrm{C}$. The resulting mixture was diluted in EtOAc. The combined organic layers were washed with saturated aqueous $\mathrm{NH}_{4} \mathrm{Cl}$ and brine, and dried over $\mathrm{MgSO}_{4}$. After filtration and concentration purification by silica gel column chromatography (hexane/EtOAc $=3: 1)$ afforded $51(53.0 \mathrm{mg}, 48 \%)$ as a colorless oil: $[\alpha]^{28} \mathrm{D}-41.6^{\circ}$ (c 1.6, $\left.\mathrm{CHCl}_{3}\right) ;{ }^{1} \mathrm{H}$ NMR $\left(500 \mathrm{MHz}, \mathrm{CDCl}_{3}\right) \delta$ 7.38-7.31 (5H, m), $6.17(1 \mathrm{H}, \mathrm{d}, J=1.7 \mathrm{~Hz}), 5.12(2 \mathrm{H}, \mathrm{s}), 3.65-3.61(1 \mathrm{H}$, m), $3.57(3 \mathrm{H}, \mathrm{s}), 3.57-3.55(1 \mathrm{H}, \mathrm{m}), 3.53(3 \mathrm{H}, \mathrm{s}), 3.49(3 \mathrm{H}, \mathrm{s}), 3.43(1 \mathrm{H}, \mathrm{dd}, J=3.4,9.5 \mathrm{~Hz}), 3.17(1 \mathrm{H}, \mathrm{dd}, J=9.5,9.5$ $\mathrm{Hz}), 2.52-2.40(3 \mathrm{H}, \mathrm{m}), 2.35-2.25(2 \mathrm{H}, \mathrm{m}), 1.28(3 \mathrm{H}, \mathrm{d}, J=6.3 \mathrm{~Hz}), 1.04(3 \mathrm{H}, \mathrm{d}, J=6.6 \mathrm{~Hz}) ;{ }^{13} \mathrm{C} \mathrm{NMR}(126 \mathrm{MHz}$, $\left.\mathrm{CDCl}_{3}\right) \delta 171.9,170.5,135.8,128.6,128.3,128.2,90.8,81.5,80.7,76.3,70.4,66.3,61.0,59.0,57.8,40.6,27.3,19.8$, 17.7; IR (ATR, $\mathrm{cm}^{-1}$ ) 2978, 2934, 2830, 1733, 1456, 1385, 1121, 1100, 1084; HRMS (ESI) calcd for $\mathrm{C}_{22} \mathrm{H}_{32} \mathrm{O}_{8} \mathrm{Na}$ $[\mathrm{M}+\mathrm{Na}]^{+}$447.1989; found $\mathrm{m} / \mathrm{z} 447.1994$. 


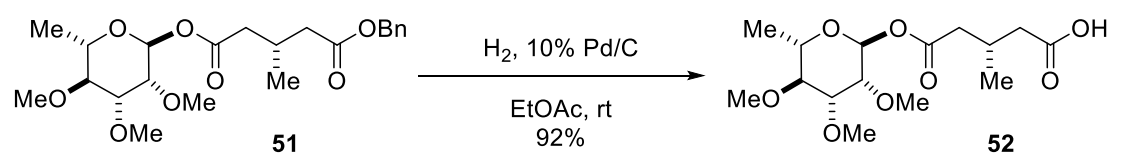

Compound 52: Compound 51 (39.8 mg, $0.094 \mathrm{mmol})$ and 10\% Pd/C (5.0 mg, $4.7 \mu \mathrm{mol})$ were dissolved in EtOAc (1.0 $\mathrm{mL}$ ) and placed under argon. The argon atmosphere was replaced with hydrogen and the mixture was stirred at room temperature for $5.5 \mathrm{~h}$. The resulting mixture was filtrated through celite and concentrated to afford $\mathbf{5 2}(28.7 \mathrm{mg}, 92 \%)$ as a colorless oil: $\left.[\alpha]^{28} \mathrm{D}-48.2^{\circ}(c) 3.5, \mathrm{CHCl}_{3}\right) ;{ }^{1} \mathrm{H}$ NMR $\left(500 \mathrm{MHz}, \mathrm{CDCl}_{3}\right) \delta 6.18(1 \mathrm{H}, \mathrm{d}, J=2.0 \mathrm{~Hz}), 3.67-3.62(1 \mathrm{H}$, m), $3.57(3 \mathrm{H}, \mathrm{s}), 3.57-3.56(1 \mathrm{H}, \mathrm{m}), 3.53(3 \mathrm{H}, \mathrm{s}), 3.51(3 \mathrm{H}, \mathrm{s}), 3.46-3.44(1 \mathrm{H}, \mathrm{dd}, J=3.2,9.5 \mathrm{~Hz}), 3.19(1 \mathrm{H}, \mathrm{dd}, J=$ 9.5, $9.5 \mathrm{~Hz}), 2.52-2.42(3 \mathrm{H}, \mathrm{m}), 2.36-2.29(2 \mathrm{H}, \mathrm{m}), 1.34(3 \mathrm{H}, \mathrm{d}, J=6.3 \mathrm{~Hz}), 1.04(3 \mathrm{H}, \mathrm{d}, J=6.3 \mathrm{~Hz}) ;{ }^{13} \mathrm{C} \mathrm{NMR}(126$ $\left.\mathrm{MHz}, \mathrm{CDCl}_{3}\right) \delta 177.6,170.5,90.8,81.5,80.7,76.2,70.4,61.0,58.9,57.8,40.5,40.2,27.1,19.7,17.7$; IR $\left(\right.$ ATR $\left.\mathrm{cm}^{-1}\right)$ 2978, 2936, 2832, 1734, 1711, 1457, 1386, 1120, 1099, 1082; HRMS (FAB) calcd for $\mathrm{C}_{15} \mathrm{H}_{27} \mathrm{O}_{8}[\mathrm{M}+\mathrm{H}]^{+} 335.1706$ found; 335.1712 .

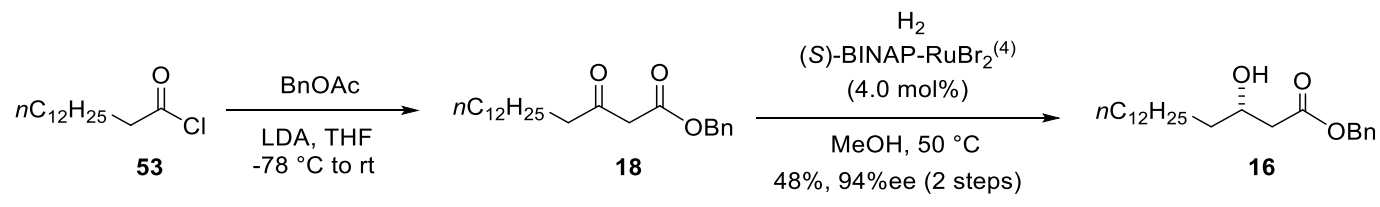

Compound 16: To a solution of diisopropylamine $(7.30 \mathrm{ml}, 51.9 \mathrm{mmol})$ in THF $(32 \mathrm{~mL})$ was added $n$-BuLi $(30.1 \mathrm{~mL}$, $48.1 \mathrm{mmol}$ ) at $-78{ }^{\circ} \mathrm{C}$ and the resultant solution was stirred at $-78{ }^{\circ} \mathrm{C}$ for $0.5 \mathrm{~h}$ and at $0{ }^{\circ} \mathrm{C}$ for $0.5 \mathrm{~h}$. After benzyl acetate $(6.85 \mathrm{~mL}, 48.1 \mathrm{mmol})$ was added, the solution was stirred for $10 \mathrm{~min}$. Myristoyl chloride $\mathbf{5 3}$ (12.0 ml, 44.4 mmol) was added to the solution at one portion, and the resultant solution was stirred at $25^{\circ} \mathrm{C}$ for $0.5 \mathrm{~h}$. The reaction was then quenched with $1 \mathrm{M}$ aqueous $\mathrm{HCl}$. After extraction with EtOAc, the combined organic layers were washed with brine and dried over $\mathrm{Na}_{2} \mathrm{SO}_{4}$. After filtration and concentration under reduced pressure, purification by silica gel column chromatography (hexane/EtOAc $=10: 1$ ) afforded $\beta$-ketoester 18 along with BnOAc.

To the mixture of the above $\beta$-ketoester 18 in $\mathrm{MeOH}(30 \mathrm{~mL})$ was placed under argon, and added $(S)$-BINAP- $\mathrm{RuBr}_{2}$ $(4.0 \mathrm{~mol} \%)$. The argon atmosphere was replaced with hydrogen and the mixture was stirred for $24 \mathrm{~h}$ at $50{ }^{\circ} \mathrm{C}$. The resulting mixture was then concentrated. Purification by silica gel column chromatography (hexane/EtOAc $=10: 1$ ) afforded 16 (6.43 g, 48\%, 94\%ee for two steps) as a colorless solid: $[\alpha]^{25} \mathrm{D}+15.7^{\circ}\left(c 1.11, \mathrm{CHCl}_{3}\right) ;{ }^{1} \mathrm{H} \mathrm{NMR}(500 \mathrm{MHz}$, $\left.\mathrm{CDCl}_{3}\right) \delta$ 7.40-7.30 (5 H , m), $5.15(2 \mathrm{H}, \mathrm{s}), 4.06-3.98(1 \mathrm{H}, \mathrm{m}), 2.83(1 \mathrm{H}, \mathrm{br} \mathrm{s}), 2.56(1 \mathrm{H}, \mathrm{dd}, J=16.6,3.2 \mathrm{~Hz}), 2.46$ $(1 \mathrm{H}, \mathrm{dd}, J=16.6,9.2 \mathrm{~Hz}), 1.56-1.50(1 \mathrm{H}, \mathrm{m}), 1.45-1.40(1 \mathrm{H}, \mathrm{m}), 1.35-1.17(22 \mathrm{H}, \mathrm{m}), 0.88(3 \mathrm{H}, \mathrm{t}, J=6.9 \mathrm{HZ}) ;{ }^{13} \mathrm{C}$ NMR $\left(126 \mathrm{MHz}, \mathrm{CDCl}_{3}\right) \delta 172.9,135.6,128.6,128.4,128.3,68.1,66.5,41.4,36.5,31.9,29.67,29.65,29.63,29.57$, 29.55, 29.50, 29.3, 25.5, 22.7, 14.1; IR (ATR, $\mathrm{cm}^{-1}$ ) 3545, 2915, 1711, 1404, 1161; HRMS (ESI) calcd for $\mathrm{C}_{23} \mathrm{H}_{38} \mathrm{O}_{3} \mathrm{Na}$ $[\mathrm{M}+\mathrm{Na}]^{+}$385.2713; found $\mathrm{m} / \mathrm{z} 385.2718$. 

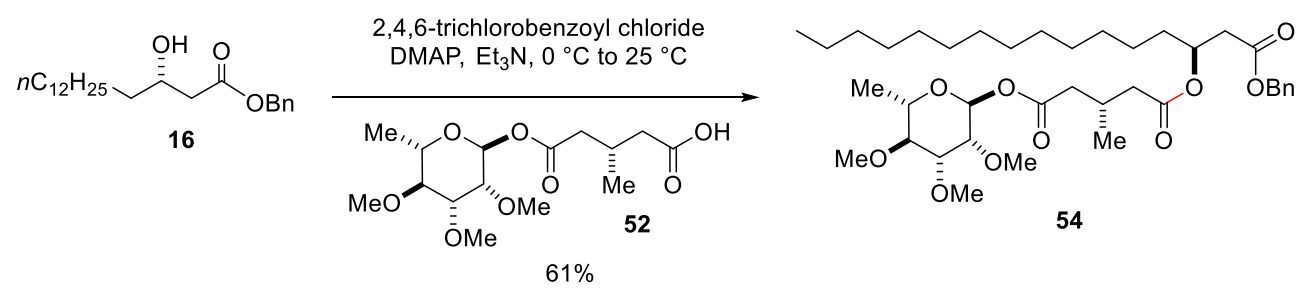

Compound 54: To a solution of carboxylic acid $52(116 \mathrm{mg}, 347 \mu \mathrm{mol})$ in $\mathrm{CH}_{2} \mathrm{Cl}_{2}(2.0 \mathrm{~mL})$ was added $\mathrm{Et}_{3} \mathrm{~N}(101 \mu \mathrm{L}$, $720 \mu \mathrm{mol})$ and 2,4,6-trichlorobenzoyl chloride $(70.0 \mu \mathrm{mol}, 448 \mu \mathrm{mol})$ at $0{ }^{\circ} \mathrm{C}$, and the resultant solution was stirred at $0{ }^{\circ} \mathrm{C}$ for $1.5 \mathrm{~h}$. The reaction mixture was added dropwise to a solution of $\mathbf{1 6}(130 \mathrm{mg}, 359 \mu \mathrm{mol})$ and DMAP ( $89.0 \mathrm{mg}$, $72.8 \mu \mathrm{mol})$ in $\mathrm{CH}_{2} \mathrm{Cl}_{2}(2.0 \mathrm{~mL})$ at $0{ }^{\circ} \mathrm{C}$. The resultant solution was then stirred at $25{ }^{\circ} \mathrm{C}$ for $1.5 \mathrm{~h}$. The reaction was then quenched with saturated aqueous $\mathrm{NH}_{4} \mathrm{Cl}$. The mixture was extracted with $\mathrm{CHCl}_{3}$, and the combined organic layers were washed with brine and dried over $\mathrm{Na}_{2} \mathrm{SO}_{4}$. Filtration and concentration under reduced pressure, the obtained residue was purified by silica gel column chromatography (hexane/EtOAc $=2: 1)$ afforded compound $54(145 \mathrm{mg}$, 61\%) as a colorless oil: $[\alpha]^{19} \mathrm{D}-24.1^{\circ}\left(c 1.04, \mathrm{CHCl}_{3}\right) ;{ }^{1} \mathrm{H} \mathrm{NMR}\left(500 \mathrm{MHz}, \mathrm{CDCl}_{3}\right) \delta 7.35-7.27(\mathrm{~m}, 5 \mathrm{H}), 6.15(\mathrm{~d}, J=$ $2.0 \mathrm{~Hz}, 1 \mathrm{H}), 5.26-5.19(\mathrm{~m}, 1 \mathrm{H}), 5.08(\mathrm{~s}, 2 \mathrm{H}), 3.63(\mathrm{dd}, J=9.5,6.1 \mathrm{~Hz}, 1 \mathrm{H}), 3.56-3.52(\mathrm{~m}, 4 \mathrm{H}), 3.50(\mathrm{~s}, 3 \mathrm{H}), 3.47$ (s, $3 \mathrm{H}), 3.43(\mathrm{dd}, J=9.4,3.4 \mathrm{~Hz}, 1 \mathrm{H}), 3.15(\mathrm{dd}, J=9.5 \mathrm{~Hz}, 1 \mathrm{H}), 2.59$ (dd, $J=9.7,6.4 \mathrm{~Hz}, 2 \mathrm{H}), 2.44-2.35$ (m, 2H), 2.29$2.12(\mathrm{~m}, 3 \mathrm{H}), 1.66-1.48(\mathrm{~m}, 2 \mathrm{H}), 1.32-1.16(\mathrm{~m}, 25 \mathrm{H}), 0.98(\mathrm{~d}, J=6.4 \mathrm{~Hz}, 3 \mathrm{H}), 0.86(\mathrm{t}, J=6.9 \mathrm{~Hz}, 3 \mathrm{H}) ;{ }^{13} \mathrm{C} \mathrm{NMR}$ $\left(126 \mathrm{MHz}, \mathrm{CDCl}_{3}\right) \delta 171.3,170.4,170.1,135.6,128.5,128.3,128.2,90.7,81.4,80.6,76.2,70.6,70.3,66.4,60.9,58.9$, 57.7, 40.7, 40.5, 39.1, 33.9, 31.8, 29.6, 29.54, 29.53, 29.50, 29.4, 29.3, 29.24, 29.21, 27.2, 25.0, 22.6, 19.6, 17.7, 14.0; HRMS (FAB) calcd for $\mathrm{C}_{38} \mathrm{H}_{63} \mathrm{O}_{10}[\mathrm{M}+\mathrm{H}]^{+} 679.4421$; found $\mathrm{m} / z$ 679.4415.

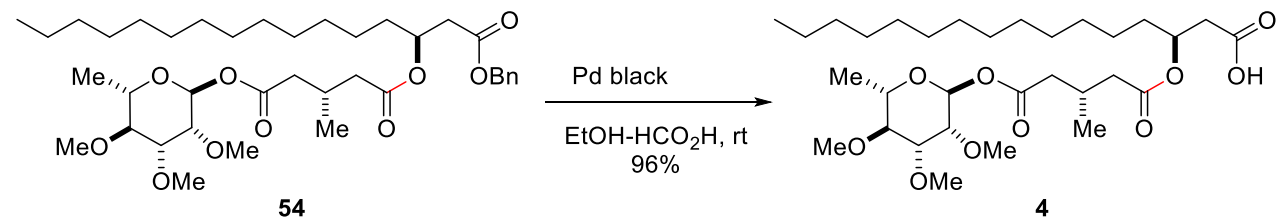

Compound 4: To a solution of $54(11.2 \mathrm{mg}, 164 \mu \mathrm{mol})$ in $\mathrm{EtOH}-\mathrm{HCO}_{2} \mathrm{H}(1.1 \mathrm{ml}, \mathrm{v} / \mathrm{v}=10: 1)$ was added Pd black (22.1 mg, $208 \mu \mathrm{mol}$ ) at $25^{\circ} \mathrm{C}$, and the resultant mixture was stirred at $25^{\circ} \mathrm{C}$ for $12.5 \mathrm{~h}$. After filtration through celite, the filtrate was concentrated under reduced pressure. The resultant residue was purified by silica gel column chromatography (hexane/EtOAc $=2: 1)$ afforded $4(9.3 \mathrm{mg}, 96 \%)$ as a colorless oil: $[\alpha]^{19} \mathrm{D}-17.6^{\circ}\left(c 1.24, \mathrm{CHCl}_{3}\right) ;{ }^{1} \mathrm{H}$ NMR $\left(500 \mathrm{MHz}, \mathrm{CDCl}_{3}\right) \delta 6.12(\mathrm{~d}, J=1.7 \mathrm{~Hz}, 1 \mathrm{H}), 5.23-5.14(\mathrm{~m}, 1 \mathrm{H}), 3.68-3.61(\mathrm{~m}, 1 \mathrm{H}), 3.57-3.53(\mathrm{~m}, 4 \mathrm{H})$, $3.52(\mathrm{~d}, J=3.4 \mathrm{~Hz}, 1 \mathrm{H}), 3.49$ (s, 3H), 3.46 (s, 3H), $3.19(\mathrm{dd}, J=9.3 \mathrm{~Hz}, 1 \mathrm{H}), 2.54(\mathrm{~m}, 2 \mathrm{H}), 2.46-2.31(\mathrm{~m}, 3 \mathrm{H}), 2.25$ (m, 2H), 2.15 (dd, $J=14.4,7.4 \mathrm{~Hz}, 1 \mathrm{H}), 1.65-1.47$ (m, 2H), $1.31-1.16(\mathrm{~m}, 25 \mathrm{H}), 0.99$ (d, $J=6.4 \mathrm{~Hz}, 3 \mathrm{H}), 0.83(\mathrm{t}, J$ $=6.9 \mathrm{~Hz}, 3 \mathrm{H}) ;{ }^{13} \mathrm{C} \mathrm{NMR}\left(126 \mathrm{MHz}, \mathrm{CDCl}_{3}\right) \delta 173.8,171.5,170.3,90.7,81.9,80.3,76.0,70.5,70.0,61.0,58.9,57.6$, $41.0,40.8,38.5,33.9,31.8,29.58,29.55,29.54,29.52,29.44,29.38,29.3,27.5,25.0,22.6,19.5,17.7,14.0$; HRMS (FAB) calcd for $\mathrm{C}_{31} \mathrm{H}_{56} \mathrm{O}_{10} \mathrm{Na}[\mathrm{M}+\mathrm{Na}]^{+} 611.3771$; found $m / z 611.3768$. 


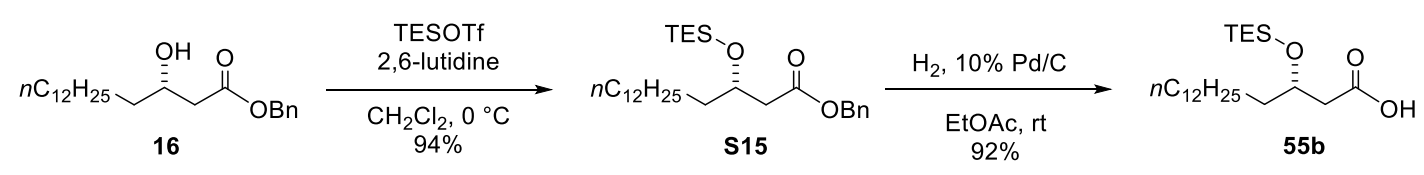

Compound 55b: To a solution of $16(282 \mathrm{mg}, 0.778 \mathrm{mmol})$ in $\mathrm{CH}_{2} \mathrm{Cl}_{2}(5.0 \mathrm{~mL})$ were added 2,6-lutidine $(0.135 \mathrm{~mL}$, $1.16 \mathrm{mmol})$ and TESOTf $(0.23 \mathrm{~mL}, 1.02 \mathrm{mmol})$ at $0{ }^{\circ} \mathrm{C}$, and the resultant solution was then stirred at $0{ }^{\circ} \mathrm{C}$ for $1 \mathrm{~h}$. The reaction was then quenched with water. After extraction with $\mathrm{CHCl}_{3}$, the combined organic layers were washed with brine and dried over $\mathrm{Na}_{2} \mathrm{SO}_{4}$. After filtration and concentration under reduced pressure, purification by silica gel column chromatography (hexane/EtOAc $=10: 1)$ afforded compound $\mathbf{S 1 5}(347 \mathrm{mg}, 94 \%)$ as a yellow oil: $[\alpha]^{25} \mathrm{D}+4.7^{\circ}$ (c 3.02, $\left.\mathrm{CHCl}_{3}\right) ;{ }^{1} \mathrm{H}$ NMR $\left(500 \mathrm{MHz}, \mathrm{CDCl}_{3}\right) \delta$ 7.38-7.29 (5H, m), $5.11(2 \mathrm{H}, \mathrm{s}), 4.18-4.12(1 \mathrm{H}, \mathrm{m}), 2.51-2.47(2 \mathrm{H}, \mathrm{m})$, $1.53-1.42(2 \mathrm{H}, \mathrm{m}), 1.37-1.20(22 \mathrm{H}, \mathrm{m}), 0.93(9 \mathrm{H}, \mathrm{t}, J=8.0 \mathrm{~Hz}), 0.88(3 \mathrm{H}, \mathrm{t}, J=7.0 \mathrm{~Hz}), 0.58(6 \mathrm{H}, \mathrm{q}, J=8.0 \mathrm{~Hz}) ;{ }^{13} \mathrm{C}$ NMR $\left(126 \mathrm{MHz}, \mathrm{CDCl}_{3}\right) \delta 171.7,135.9,128.5,128.3,128.2$, 69.4, 66.2, 42.8, 37.7, 31.9, 29.7, 29.64, 29.57, 29.4, 25.1, 22.7, 14.1, 6.8, 4.9; IR (ATR, $\mathrm{cm}^{-1}$ ) 1739, 1456, 1379, 790, 742; HRMS (ESI) calcd for $\mathrm{C}_{29} \mathrm{H}_{52} \mathrm{O}_{3} \mathrm{SiNa}[\mathrm{M}+\mathrm{Na}]^{+}$ 499.3578; found $m / z 499.3576$.

To a solution of the above S15 (307 mg, $0.644 \mathrm{mmol})$ in EtOAc $(13 \mathrm{~mL})$ was added 10\% Pd/C (66.3 mg, $62.3 \mu \mathrm{mol})$ at $25^{\circ} \mathrm{C}$, and the reaction mixture was stirred at $25^{\circ} \mathrm{C}$ for $1.5 \mathrm{~h}$. After filtration and concentration, the obtained residue was purified by silica gel column chromatography (hexane/EtOAc $=0: 1)$ afforded $\mathbf{5 5 b}(230 \mathrm{mg}, 92 \%)$ as a yellow oil: $[\alpha]^{25} \mathrm{D}-1.4^{\circ}\left(c \mathrm{c} 0.68, \mathrm{CHCl}_{3}\right) ;{ }^{1} \mathrm{H} \mathrm{NMR}\left(500 \mathrm{MHz}, \mathrm{CDCl}_{3}\right) \delta 4.16-4.05(1 \mathrm{H}, \mathrm{m}), 2.56(1 \mathrm{H}, \mathrm{dd}, J=15.2,5.2 \mathrm{~Hz}), 2.49$ $(1 \mathrm{H}, \mathrm{dd}, J=15.2,5.7 \mathrm{~Hz}), 1.59-1.49(2 \mathrm{H}, \mathrm{m}), 1.36-1.21(22 \mathrm{H}, \mathrm{m}), 0.97(9 \mathrm{H}, \mathrm{t}, J=8.2 \mathrm{~Hz}), 0.88(3 \mathrm{H}, \mathrm{t}, J=7.0 \mathrm{~Hz})$, $0.64(6 \mathrm{H}, \mathrm{q}, J=8.0 \mathrm{~Hz}):{ }^{13} \mathrm{C} \mathrm{NMR}\left(126 \mathrm{MHz}, \mathrm{CDCl}_{3}\right) \delta 174.9,69.4,41.5,37.2,31.9,29.7,29.6,29.6,29.5,29.5,29.4$, 25.3, 22.7, 14.1, 6.7, 4.8; IR (ATR, $\mathrm{cm}^{-1}$ ) 2923, 1712, 1457, 1098, 1007; HRMS (ESI) calcd for $\mathrm{C}_{22} \mathrm{H}_{46} \mathrm{O}_{3} \mathrm{SiNa}$ $[\mathrm{M}+\mathrm{Na}]^{+}$409.3108; found $\mathrm{m} / \mathrm{z} 409.3098$.

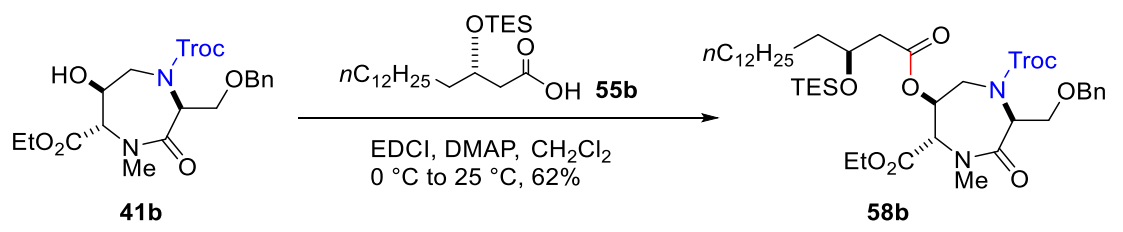

Compound 58b: To a solution of $41 \mathbf{b}(5.2 \mathrm{mg}, 10.2 \mu \mathrm{mol})$ and carboxylic acid $55 \mathrm{~b}(8.0 \mathrm{mg}, 20.7 \mu \mathrm{mol})$ in $\mathrm{CH}_{2} \mathrm{Cl}_{2}$ $(1.0 \mathrm{~mL})$ were added DMAP $(7.8 \mathrm{mg}, 64 \mu \mathrm{mol})$ and EDCI $(4.8 \mathrm{mg}, 25 \mu \mathrm{mol})$ at $0{ }^{\circ} \mathrm{C}$, and the resultant solution was stirred at $25^{\circ} \mathrm{C}$ for $1.5 \mathrm{~h}$. The reaction was then quenched with saturated aqueous $\mathrm{NH}_{4} \mathrm{Cl}$. The mixture was extracted with $\mathrm{CHCl}_{3}$, and the combined organic layers were washed with brine and dried over $\mathrm{Na}_{2} \mathrm{SO}_{4}$. Filtration and concentration under reduced pressure afforded a coupling product $\mathbf{5 8 b}(5.6 \mathrm{mg}, 62 \%)$ which was used for the next reaction immediately. (The acylated product was unstable under the basic conditions.): ${ }^{1} \mathrm{H} \mathrm{NMR}\left(500 \mathrm{MHz}, \mathrm{CDCl}_{3}\right) \delta$ $7.35-7.13(\mathrm{~m}, 5 \mathrm{H}), 5.92-5.75(\mathrm{~m}, 1 \mathrm{H}), 4.85(\mathrm{~m}, J=23.3,11.7 \mathrm{~Hz}, 1 \mathrm{H}), 4.72(\mathrm{br}, 1 \mathrm{H}), 4.59$ (dd, $J=16.7,12.3 \mathrm{~Hz}$, $1 \mathrm{H}), 4.48(\mathrm{~d}, J=12.3 \mathrm{~Hz}, 1 \mathrm{H}), 4.46-4.33(\mathrm{~m}, 2 \mathrm{H}), 4.29(\mathrm{~m}, 1 \mathrm{H}), 4.28-4.15(\mathrm{~m}, 3 \mathrm{H}), 4.15-4.03(\mathrm{~m}, 2 \mathrm{H}), 3.77$ (dd, $J=9.9,3.5 \mathrm{~Hz}, 1 \mathrm{H}), 3.29(\mathrm{~m}, 1 \mathrm{H}), 3.20-3.08(\mathrm{~m}, 4 \mathrm{H}), 2.50-2.42(\mathrm{~m}, 2 \mathrm{H}), 1.52(\mathrm{~m}, 6 \mathrm{H}), 1.38-1.13(\mathrm{~m}, 25), 1.01-$ $0.83(\mathrm{~m}, 14 \mathrm{H}), 0.69-0.49(\mathrm{~m}, 6 \mathrm{H}) ;{ }^{13} \mathrm{C} \mathrm{NMR}\left(126 \mathrm{MHz}, \mathrm{CDCl}_{3}\right) \delta 170.7,169.1,166.7,152.9,138.1,128.37,128.34$, $127.58,127.53,127.49,75.7,73.4,62.7,42.3,37.7,31.9,30.3,29.67,29.64,29.59,29.56,29.4,25.2,22.7,14.16$, 14.15, 14.13, 14.07, 6.9, 4.9; HRMS (FAB) calcd for $\mathrm{C}_{42} \mathrm{H}_{70} \mathrm{Cl}_{3} \mathrm{~N}_{2} \mathrm{O}_{9}[\mathrm{M}+\mathrm{H}]^{+}$879.3916; found $\mathrm{m} / z$ 879.3914. 


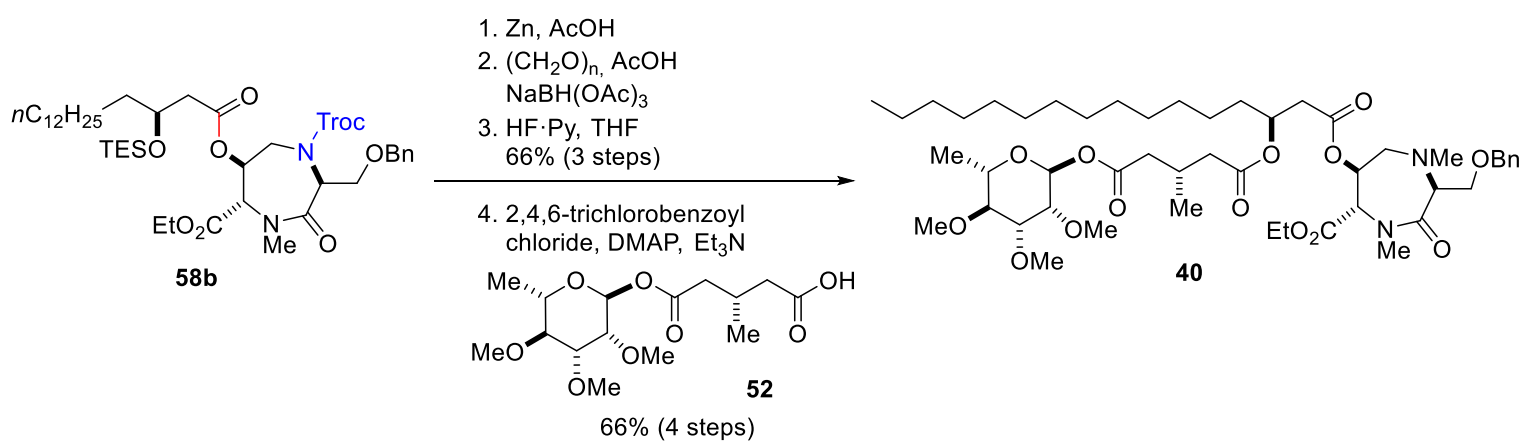

Compound 40: To a solution of the above coupling product 58b $(3.3 \mathrm{mg})$ in THF $(1.5 \mathrm{~mL})$ were added $\mathrm{Zn}$ powder (60.0 mg, $0.917 \mathrm{mmol})$ and $\mathrm{AcOH}(100 \mu 1,1.75 \mathrm{mmol})$ at $25^{\circ} \mathrm{C}$, and the resultant solution was stirred at $60{ }^{\circ} \mathrm{C}$ for 12 h. The reaction was then quenched with saturated aqueous $\mathrm{NaHCO}_{3}$. After extraction with $\mathrm{CHCl}_{3}$, the combined organic layers were washed with brine and dried over $\mathrm{Na}_{2} \mathrm{SO}_{4}$. Filtration and concentration under reduced pressure afforded a crude product $(2.0 \mathrm{mg})$ which was used for the next reaction without further purification.

To a solution of the above crude amine $(2.0 \mathrm{mg})$ in dichloroethane $(1.5 \mathrm{~mL})$ were added paraformaldehyde $(8.3 \mathrm{mg}$, $0.277 \mathrm{mmol}), \mathrm{AcOH}(20 \mu \mathrm{L}, 0.35 \mathrm{mmol})$ and $\mathrm{NaBH}(\mathrm{OAc})_{3}(6.4 \mathrm{mg}, 30.2 \mu \mathrm{mol})$ at $25^{\circ} \mathrm{C}$, and the resultant solution was stirred at $25{ }^{\circ} \mathrm{C}$ for $4 \mathrm{~h}$. The reaction was then quenched with saturated aqueous $\mathrm{NaHCO}_{3}$. After extraction with $\mathrm{CHCl}_{3}$, the combined organic layers were washed with brine and dried over $\mathrm{Na}_{2} \mathrm{SO}_{4}$. Filtration and concentration under reduced pressure afforded a crude methylated product $(1.5 \mathrm{mg})$ which was used for the next reaction without further purification.

To a solution of the above crude methylated product $(1.5 \mathrm{mg})$ in THF $(1.0 \mathrm{~mL})$ was added HF $p$ pyr $(20.0 \mu \mathrm{L}, 222$ $\mu \mathrm{mol}$ ) at $0{ }^{\circ} \mathrm{C}$, and the resultant solution was stirred at $25^{\circ} \mathrm{C}$ for $9 \mathrm{~h}$. The reaction was then quenched with saturated aqueous $\mathrm{NaHCO}_{3}$. After extraction with $\mathrm{CHCl}_{3}$, the combined organic layers were washed with brine and dried over $\mathrm{Na}_{2} \mathrm{SO}_{4}$. After filtration and concentration under reduced pressure, purification by flash silica gel column chromatography (hexane/EtOAc $=2: 1)$ afforded a crude product $59(1.5 \mathrm{mg}$,) as a white amorphous.

To a solution of carboxylic acid $\mathbf{5 2}(8.1 \mathrm{mg}, 24 \mu \mathrm{mol})$ in $\mathrm{CH}_{2} \mathrm{Cl}_{2}(1.0 \mathrm{~mL})$ was added $\mathrm{Et}_{3} \mathrm{~N}(6.0 \mu \mathrm{L}, 43 \mu \mathrm{mol})$ and 2,4,6-trichlorobenzoyl chloride $(4.0 \mu \mathrm{mol}, 26 \mu \mathrm{mol})$ at $0{ }^{\circ} \mathrm{C}$, and the resultant solution was stirred at $0{ }^{\circ} \mathrm{C}$ for $1 \mathrm{~h}$. The reaction mixture was added dropwise to a solution of the above crude compound $\mathbf{5 9}(1.5 \mathrm{mg})$ and DMAP (6.6 mg, 54 $\mu \mathrm{mol})$ in $\mathrm{CH}_{2} \mathrm{Cl}_{2}(2.5 \mathrm{~mL})$ at $0{ }^{\circ} \mathrm{C}$. The resultant solution was then stirred at $25^{\circ} \mathrm{C}$ for $1 \mathrm{~h}$. The reaction mixture was directly purified by silica gel column chromatography (hexane/EtOAc $=2: 1)$ afforded compound $\mathbf{4 0}(2.3 \mathrm{mg}, 66 \%$ for four steps) as a colorless oil: ${ }^{1} \mathrm{H}$ NMR $\left(500 \mathrm{MHz}, \mathrm{CDCl}_{3}\right) \delta 7.32(5 \mathrm{H}, \mathrm{m}), 6.17(2 \mathrm{H}, \mathrm{m}), 5.53(1 \mathrm{H}, \mathrm{br}), 5.19(1 \mathrm{H}, \mathrm{br})$, $4.58(1 \mathrm{H}, \mathrm{d}, J=12.4 \mathrm{~Hz}), 4.54(1 \mathrm{H}, \mathrm{d}, J=12.0 \mathrm{~Hz}), 4.32-4.18(4 \mathrm{H}, \mathrm{m}), 3.94-3.82(2 \mathrm{H}, \mathrm{m}), 3.66-3.59(2 \mathrm{H}, \mathrm{m})$, 3.59-3.48 (9H, m), $3.45(2 \mathrm{H}, \mathrm{dd}, J=8.2,2.4 \mathrm{~Hz}), 3.28-3.10(4 \mathrm{H}, \mathrm{m}), 3.07(3 \mathrm{H}, \mathrm{s}), 2.59-2.16(12 \mathrm{H}, \mathrm{m}), 1.33-1.21(25 \mathrm{H}$, m), 1.03-1.02 (3H, m), 0.91-0.86 (5H, m); ${ }^{13} \mathrm{C}$ NMR (126 MHz, $\left.\mathrm{CDCl}_{3}\right) \delta 128.4,127.8,127.6,90.8,81.5,80.7,76.4$, 73.4, 70.5, 67.7, 64.5, 61.2, 59.2, 57.9, 40.8, 39.3, 34.1, 32.0, 29.8, 29.7, 29.6, 29.5, 27.5, 25.3, 22.7, 19.8, 18.0, 14.2; IR $\left(\right.$ ATR, $\left.\mathrm{cm}^{-1}\right) 3380,2928,2360,1743,1457,1292,1164,1123,1100$; HRMS (ESI) calcd for $\mathrm{C}_{49} \mathrm{H}_{81} \mathrm{~N}_{2} \mathrm{O}_{14}[\mathrm{M}+\mathrm{H}]^{+}$ 921.5682; found: $\mathrm{m} / \mathrm{z} 921.5664$. 

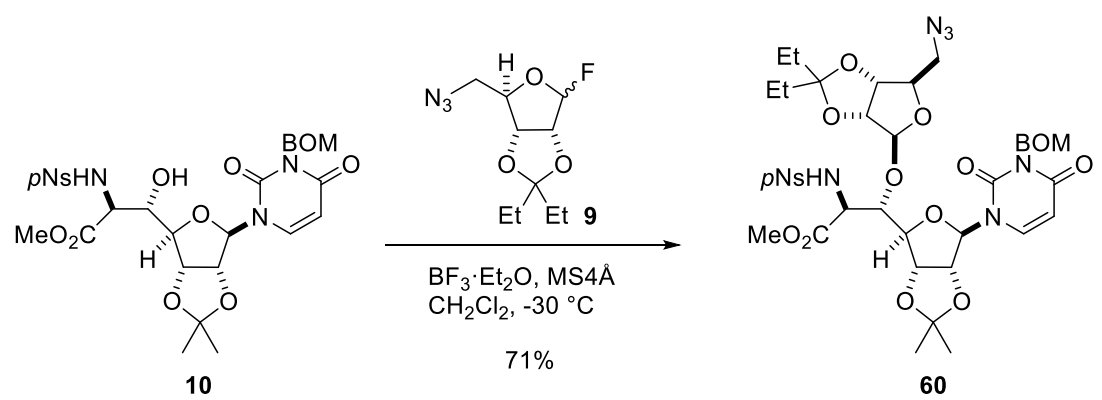

Compound 60: To a solution of $\mathbf{1 0}(1.92 \mathrm{~g}, 2.84 \mathrm{mmol})$, aminoribose $\mathbf{9}^{9}(1.13 \mathrm{~g}, 4.61 \mathrm{mmol})$ and $\mathrm{MS} 4 \AA \mathrm{A}(2.71 \mathrm{~g})$ in $\mathrm{CH}_{2} \mathrm{Cl}_{2}\left(30 \mathrm{~mL}\right.$ ) was added $\mathrm{BF}_{3} \cdot \mathrm{Et}_{2} \mathrm{O}$ (four injections every $1 \mathrm{~h}, 188 \mu \mathrm{L} \mathrm{x} 4,1.49 \mathrm{mmol} \mathrm{x} 4$ ) at $-30{ }^{\circ} \mathrm{C}$, and the resultant solution was stirred for $6 \mathrm{~h}$. The reaction was then quenched with saturated aqueous $\mathrm{NaHCO}_{3}$. The mixture was extracted with $\mathrm{CHCl}_{3}$, the combined organic layers were washed with brine and dried over $\mathrm{Na}_{2} \mathrm{SO}_{4}$. After filtration and concentration under reduced pressure, purification by silica gel column chromatography (hexane/EtOAc $=1: 1$ ) afforded 60 (1.81 g, 71\%) as a yellow amorphous (no noticeable amount of $\alpha$-anomer was obtained): $[\alpha]^{25} \mathrm{D}+27.0^{\circ}(c$ $\left.0.99, \mathrm{CHCl}_{3}\right) ;{ }^{1} \mathrm{H}$ NMR $\left(500 \mathrm{MHz}, \mathrm{CDCl}_{3}\right) \delta 8.33(2 \mathrm{H}, \mathrm{d}, J=9.2 \mathrm{~Hz}), 8.06(2 \mathrm{H}, \mathrm{d}, J=9.2 \mathrm{~Hz}), 7.39-7.25(6 \mathrm{H}, \mathrm{m})$, 6.04-6.02 (1H, br m), $5.75(1 \mathrm{H}, \mathrm{d}, J=8.0 \mathrm{~Hz}), 5.55(1 \mathrm{H}, \mathrm{d}, J=1.7 \mathrm{~Hz}), 5.49(1 \mathrm{H}, \mathrm{d}, J=10.0 \mathrm{~Hz}), 5.41(1 \mathrm{H}, \mathrm{d}, J=$ $10.0 \mathrm{~Hz}), 5.07(1 \mathrm{H}, \mathrm{dd}, J=6.6,2.0 \mathrm{~Hz}), 5.00(1 \mathrm{H}, \mathrm{s}), 4.94(1 \mathrm{H}, \mathrm{dd}, J=6.6,4.3 \mathrm{~Hz}), 4.72-4.68(3 \mathrm{H}, \mathrm{m}), 4.57(1 \mathrm{H}, \mathrm{d}, J$ $=6.3 \mathrm{~Hz}), 4.52(1 \mathrm{H}, \mathrm{d}, J=6.3 \mathrm{~Hz}), 4.41(1 \mathrm{H}, \mathrm{dd}, J=8.3,1.4 \mathrm{~Hz}), 4.36(1 \mathrm{H}, \mathrm{br} \mathrm{s}), 4.25(1 \mathrm{H}, \mathrm{dd}, J=4.0,8.0 \mathrm{~Hz}), 4.22$ $(1 \mathrm{H}, \mathrm{dd}, J=5.8,5.8 \mathrm{~Hz}), 3.58(3 \mathrm{H}, \mathrm{s}), 3.31(1 \mathrm{H}, \mathrm{d}, J=5.5 \mathrm{~Hz}), 1.59-1.54(7 \mathrm{H}, \mathrm{m}), 1.37(3 \mathrm{H}, \mathrm{s}), 0.8(3 \mathrm{H}, \mathrm{t}, J=7.5 \mathrm{~Hz})$, $0.76(3 \mathrm{H}, \mathrm{t}, J=7.5 \mathrm{~Hz}) ;{ }^{13} \mathrm{C}$ NMR $\left(126 \mathrm{MHz}, \mathrm{CDCl}_{3}\right) \delta 169.3,162.4,150.9,150.0,146.0,141.4,137.7,128.3,127.7$, 127.7, 124.0, 123.7, 117.1, 114.9, 112.1, 102.3, 96.0, 86.4, 85.9, 85.2, 83.9, 81.7, 81.2, 79.6, 72.4, 70.3, 56.3, 53.1, 53.0, 29.3, 28.7, 27.0, 25.3, 8.3, 7.2; IR (ATR, $\left.\mathrm{cm}^{-1}\right)$ 2977, 2942, 2106, 1751, 1721, 1669, 1528, 1458, 1354, 1165, 1091; HRMS (FAB) calcd for $\mathrm{C}_{39} \mathrm{H}_{48} \mathrm{~N}_{7} \mathrm{O}_{16} \mathrm{~S}[\mathrm{M}+\mathrm{H}]^{+}$902.2878; found $\mathrm{m} / z$ 902.2888.

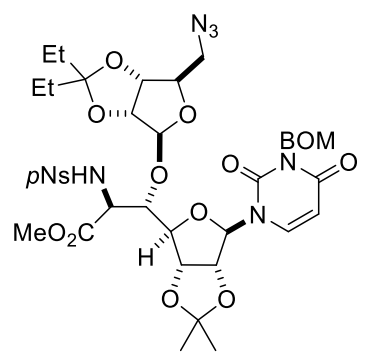

60

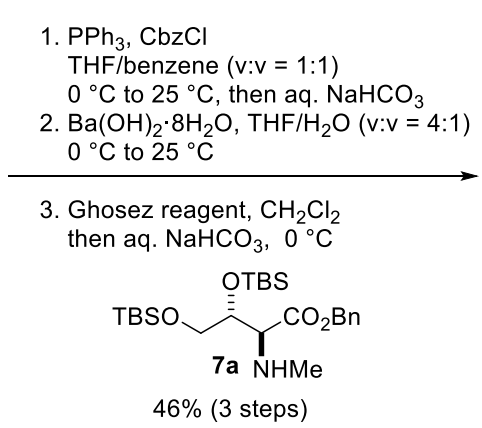

$\mathrm{PPh}_{3}, \mathrm{CbzC}$ $0{ }^{\circ} \mathrm{C}$ to $25^{\circ} \mathrm{C}$, then aq. $\mathrm{NaHCO}_{3}$

2. $\mathrm{Ba}(\mathrm{OH})_{2} \cdot 8 \mathrm{H}_{2} \mathrm{O}, \mathrm{THF} / \mathrm{H}_{2} \mathrm{O}(\mathrm{v}: \mathrm{v}=4: 1)$

3. Ghosez reagent, $\mathrm{CH}_{2} \mathrm{Cl}_{2}$

OTBS

$46 \%$ (3 steps)

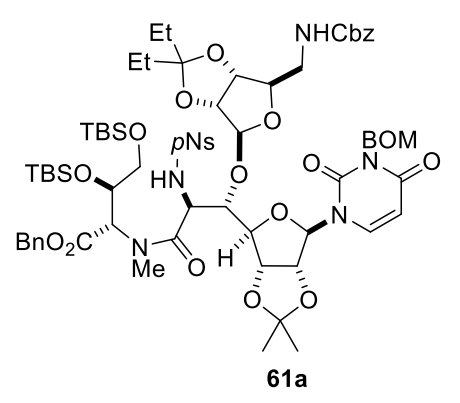

Compound 61a: To a solution of 60 (3.26 g, $3.61 \mathrm{mmol})$ in THF/benzene $(60 \mathrm{~mL}, \mathrm{v} / \mathrm{v}=1: 1)$ were added $\mathrm{CbzCl}(6.00$ $\mathrm{mL}, 42.0 \mathrm{mmol})$ and $\mathrm{PPh}_{3}(5.19 \mathrm{~g}, 19.8 \mathrm{mmol})$ at $0{ }^{\circ} \mathrm{C}$, and the resultant solution was stirred at $25{ }^{\circ} \mathrm{C}$ for $3 \mathrm{~h}$. The reaction was then quenched with saturated aqueous $\mathrm{NaHCO}_{3}$. The mixture was extracted with EtOAc, and the combined organic layers were washed with brine and dried over $\mathrm{Na}_{2} \mathrm{SO}_{4}$. After filtration and concentration under reduced pressure, purification by flash silica gel column chromatography (hexane/EtOAc $=1: 1$ ) afforded the crude product. (It was difficult to remove triphenylphosphine oxide.)

To a solution of the above crude product in $\mathrm{THF} / \mathrm{H}_{2} \mathrm{O}(35 \mathrm{~mL}, \mathrm{v} / \mathrm{v}=4: 1)$ was added $\mathrm{Ba}(\mathrm{OH})_{2} \cdot 8 \mathrm{H}_{2} \mathrm{O}(1.38 \mathrm{~g}, 4.37$ 
mmol) at $0{ }^{\circ} \mathrm{C}$, and the resultant solution was stirred at $25{ }^{\circ} \mathrm{C}$ for $5.5 \mathrm{~h}$. The reaction was then quenched with $1 \mathrm{M}$ aqueous $\mathrm{HCl}$. After extraction with $\mathrm{CHCl}_{3}$, the combined organic layers were washed with brine and dried over $\mathrm{Na}_{2} \mathrm{SO}_{4}$. After filtration and concentration under reduced pressure, purification by flash silica gel column chromatography $\left(\mathrm{CHCl}_{3} / \mathrm{MeOH}=10: 1\right)$ afforded a crude carboxylic acid $\mathbf{8}$.

To a solution of the above crude carboxylic acid 8 and $\mathrm{NaHCO}_{3}(149 \mathrm{mg}, 1.77 \mathrm{mmol})$ in $\mathrm{CH}_{2} \mathrm{Cl}_{2}(33 \mathrm{~mL})$ was added Ghosez reagent (1-chloro- $N, N, 2$-trimethylpropenylamine, $420 \mu \mathrm{L}, 3.18 \mathrm{mmol}$ ) at $0{ }^{\circ} \mathrm{C}$, and the resultant solution was then stirred at $0{ }^{\circ} \mathrm{C}$ for $1 \mathrm{~h}$. The reaction mixture was added dropwise to a solution of amine 7a and $\mathrm{NaHCO}_{3}(390 \mathrm{mg}$, $4.64 \mathrm{mmol})$ in $\mathrm{CH}_{2} \mathrm{Cl}_{2} / \mathrm{H}_{2} \mathrm{O}(40 \mathrm{~mL}, \mathrm{v} / \mathrm{v}=3: 1)$ at $0{ }^{\circ} \mathrm{C}$ for $20 \mathrm{~min}$, and the resultant mixture was stirred at $0{ }^{\circ} \mathrm{C}$ for 0.5 h. The mixture was extracted with $\mathrm{CH}_{2} \mathrm{Cl}_{2}$, the combined organic layers were washed with brine and dried over $\mathrm{Na}_{2} \mathrm{SO}_{4}$. After filtration and concentration under reduced pressure, purification by silica gel column chromatography (hexane/EtOAc $=3: 2)$ afforded $61 \mathrm{a}\left(2.40 \mathrm{~g}, 46 \%\right.$ for three steps) as a yellow amorphous: $[\alpha]^{25} \mathrm{D}+28.7^{\circ}(c) 1.75$, $\left.\mathrm{CHCl}_{3}\right) ;{ }^{1} \mathrm{H} \mathrm{NMR}\left(500 \mathrm{MHz}, \mathrm{CDCl}_{3}\right.$ at $\left.60{ }^{\circ} \mathrm{C}\right) \delta 8.10-8.08(2 \mathrm{H}, \mathrm{m}), 7.94-7.80(2 \mathrm{H}, \mathrm{m}), 7.47-7.21(16 \mathrm{H}, \mathrm{m}), 6.77-6.75$ $(1 \mathrm{H}, \mathrm{br} \mathrm{s}), 6.47-6.27(1 \mathrm{H}, \mathrm{br} \mathrm{s}), 5.75(1 \mathrm{H}, \mathrm{d}, J=8.0 \mathrm{~Hz}), 5.63(1 \mathrm{H}, \mathrm{d}, J=1.8 \mathrm{~Hz}), 5.50(1 \mathrm{H}, \mathrm{d}, J=9.4 \mathrm{~Hz}), 5.47(1 \mathrm{H}, \mathrm{d}$, $J=9.4 \mathrm{~Hz}), 5.43-5.41(1 \mathrm{H}, \mathrm{br} \mathrm{m}), 5.30(1 \mathrm{H}, \mathrm{d}, J=12.0 \mathrm{~Hz}), 5.24-5.16(1 \mathrm{H}, \mathrm{m}), 5.07(1 \mathrm{H}, \mathrm{dd}, J=6.3,1.9 \mathrm{~Hz}), 5.01$ $(2 \mathrm{H}, \mathrm{s}), 4.86(1 \mathrm{H}, \mathrm{s}), 4.72-4.59(3 \mathrm{H}, \mathrm{m}), 4.36(1 \mathrm{H}, \mathrm{d}, J=6.3 \mathrm{~Hz}), 4.29-4.22(1 \mathrm{H}, \mathrm{br} \mathrm{m}), 4.13(1 \mathrm{H}, \mathrm{dd}, J=8.0,1.7 \mathrm{~Hz})$, 4.07-4.05 (1H, br m), 3.69-3.61 (2H, m), 3.57-3.46 (2H, m), $3.20(3 \mathrm{H}, \mathrm{s}), 2.98-2.90(1 \mathrm{H}, \mathrm{m}), 1.56-1.34(10 \mathrm{H}, \mathrm{m})$, 0.90-0.69 (24H, m), 0.04-0.03 (6H, m), -0.04--0.06 (6H, m); ${ }^{13} \mathrm{C} \mathrm{NMR} \mathrm{(126} \mathrm{MHz,} \mathrm{CDCl}_{3}$ at $\left.60{ }^{\circ} \mathrm{C}\right) \delta 169.7,168.4$, 162.2, 156.7 151.1, 150.0, 141.3, 138.1, 135.4, 128.7-127.4 (11C), 123.8 (2C), 116.3, 115.1, 112.3, 102.7, 95.5, 88.6, 86.6, 86.4, 84.3, 82.0, 80.9, 78.9, 75.2, 72.5, 70.6, 66.8, 64.4, 58.5, 53.2, 43.0, 33.2, 31.5, 29.4, 28.9, 27.2, 25.8 (2C), 25.7 (2C), 25.6, 22.6, 18.2, 18.1, 17.9, 13.9, 8.3, 7.2, -3.4, -4.9 , -5.6; IR (ATR, $\mathrm{cm}^{-1}$ ) 3316, 2936, 1721, 1667, 1531, 1454, 1350, 1260, 1171, 1090, 837, 737; HRMS (ESI) calcd for $\mathrm{C}_{70} \mathrm{H}_{97} \mathrm{~N}_{6} \mathrm{O}_{21} \mathrm{Si}_{2} \mathrm{~S}[\mathrm{M}+\mathrm{H}]^{+}$1445.5961; found $\mathrm{m} / \mathrm{z}$ 1445.5981 .
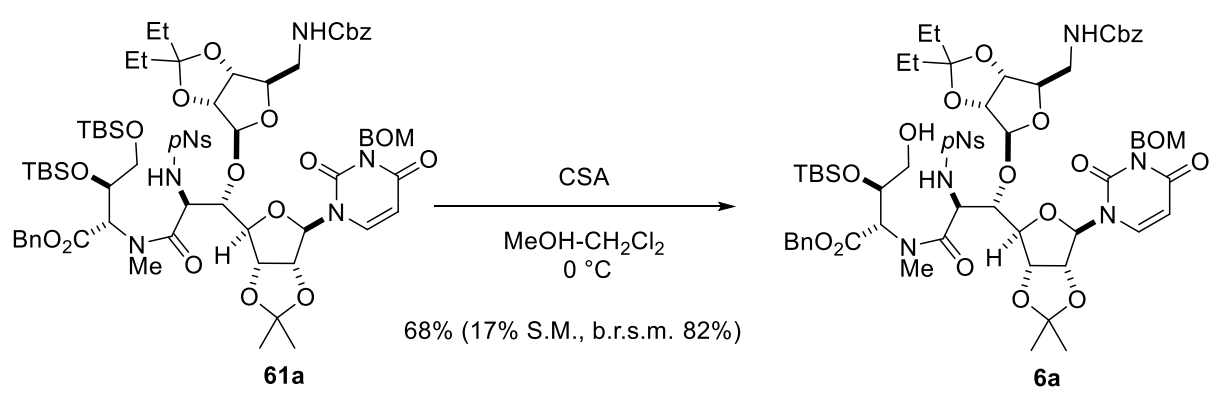

Compound 6a: To a solution of 61a (2.30 g, $1.59 \mathrm{mmol})$ in $\mathrm{CH}_{2} \mathrm{Cl}_{2}-\mathrm{MeOH}(54 \mathrm{~mL}, \mathrm{v} / \mathrm{v}=1: 1)$ was added CSA (872 $\mathrm{mg}, 3.75 \mathrm{mmol}$ ) at $0{ }^{\circ} \mathrm{C}$, and the resultant solution was stirred at $0{ }^{\circ} \mathrm{C}$ for $18 \mathrm{~h}$. The reaction was then quenched with saturated aqueous $\mathrm{NaHCO}_{3}$. The mixture was extracted with $\mathrm{CHCl}_{3}$, and the combined organic layers were washed with brine and dried over $\mathrm{Na}_{2} \mathrm{SO}_{4}$. After filtration and concentration under reduced pressure, purification by silica gel column chromatography (hexane/EtOAc $=3: 2)$ afforded 6a $(1.45 \mathrm{~g}, 68 \%$ (b.r.s.m. $82 \%)$ ) as a yellow amorphous and starting material 61a (383 mg, 17\%) as a yellow amorphous: $[\alpha]^{25} \mathrm{D}+14.3^{\circ}\left(c 1.89, \mathrm{CHCl}_{3}\right) ;{ }^{1} \mathrm{H}$ NMR $(500 \mathrm{MHz}$, $\mathrm{CDCl}_{3}$ at $25^{\circ} \mathrm{C}$; mixture of rotamers) $\delta 8.15-8.03(1 \mathrm{H}, \mathrm{m}), 7.97(1 \mathrm{H}, \mathrm{d}, J=8.6 \mathrm{~Hz}), 7.79(1 \mathrm{H}, \mathrm{d}, J=8.0 \mathrm{~Hz}), 7.56(1 \mathrm{H}$, d, $J=8.0 \mathrm{~Hz}), 7.52-7.16(16 \mathrm{H}, \mathrm{m}), 6.81-6.68(1 \mathrm{H}, \mathrm{m}), 6.43(0.6 \mathrm{H}, \mathrm{d}, J=10.3 \mathrm{~Hz}), 6.21(0.4 \mathrm{H}, \mathrm{d}, J=10.3 \mathrm{~Hz})$, 5.81-5.70 $(1 \mathrm{H}, \mathrm{m}), 5.60-5.53(1 \mathrm{H}, \mathrm{m}), 5.53-5.21(4 \mathrm{H}, \mathrm{m}), 5.18-4.95(3 \mathrm{H}, \mathrm{m}), 4.90-4.80(1 \mathrm{H}, \mathrm{m}), 4.76-4.52(4 \mathrm{H}, \mathrm{m})$, 
4.51-4.26 (2H, m), 4.22-4.09 (1H, m), 4.08-3.97 (1H, m), 3.62-3.35 $(2 \mathrm{H}, \mathrm{m}), 3.27-3.14(2 \mathrm{H}, \mathrm{m}), 3.14-3.00(2 \mathrm{H}, \mathrm{m})$, $2.94(3 \mathrm{H}, \mathrm{s}), 2.92-2.79(2 \mathrm{H}, \mathrm{m}), 2.61(1 \mathrm{H}, \mathrm{s}), 2.02-1.95(1 \mathrm{H}, \mathrm{br} \mathrm{m}), 1.64-1.24(7 \mathrm{H}, \mathrm{m}), 1.12-1.11(3 \mathrm{H}, \mathrm{m}), 0.94-0.65$ $(15 \mathrm{H}, \mathrm{m}), 0.12-\square 0.05(6 \mathrm{H}, \mathrm{m}) ;{ }^{1} \mathrm{H}$ NMR $\left(500 \mathrm{MHz}, \mathrm{CDCl}_{3}\right.$ at $60{ }^{\circ} \mathrm{C}$; mixture of rotamers) $\delta 8.13-7.56$ (4H, m), 7.44-7.15 (16H, m), 6.62-6.60 (1H, br m), 6.32-6.12 (1H, br m), $6.31(0.6 \mathrm{H}$, br m), $6.10(0.4 \mathrm{H}, \mathrm{br} \mathrm{m}), 5.73(1 \mathrm{H}, \mathrm{m})$, $5.57(1 \mathrm{H}, \mathrm{s}), 5.47(1 \mathrm{H}, \mathrm{d}, J=9.7 \mathrm{~Hz}), 5.43-5.37(2 \mathrm{H}, \mathrm{m}), 5.32-5.16(2 \mathrm{H}, \mathrm{m}), 5.14-5.04(3 \mathrm{H}, \mathrm{m}), 5.03-4.97(1 \mathrm{H}, \mathrm{m})$, 4.91-4.85 (1H, s), 4.76-4.51 (5H, m), 4.40-4.28 (2H, m), 4.22-4.16 (1H, m), 4.12-4.05 (2H, m), 3.65-3.37 (2H, m), 3.29-3.22 (1H, m), $3.16(2 \mathrm{H}, \mathrm{s}), 3.09-2.90(3 \mathrm{H}, \mathrm{m}), 2.81(1 \mathrm{H}, \mathrm{m}), 2.66-2.64(1 \mathrm{H}, \mathrm{br}), 1.54-1.26(7 \mathrm{H}, \mathrm{m}), 1.13-1.10(3 \mathrm{H}$, m), 0.90-0.68 (15H, m), 0.07--0.02 (6H, m); ${ }^{13} \mathrm{C} \mathrm{NMR} \mathrm{(126} \mathrm{MHz,} \mathrm{CDCl}_{3}$ at $60{ }^{\circ} \mathrm{C}$; mixture of rotamers) $\delta 168.4,162.3$, $162.1,156.5,151.1,150.9,150.3,149.9,145.6,142.0,141.7,137.9,137.5,137.3,135.4,134.9,128.7,128.6,128.6$, $128.5,128.4,128.4,128.4,128.3,128.2,127.9,127.8,127.8,127.7,127.7,127.6,123.9,123.6,116.2,115.9,115.0$, $112.4,102.8,102.3,96.4,96.11,88.57,88.3,88.3,86.8,86.6,86.5,86.4,84.3,81.9,81.0,78.7,78.5,72.4,72.29$, $71.6,70.5,70.4,67.7,66.7,66.5,63.6,62.2,60.9,60.5,54.2,53.4,43.3,43.1,34.0,31.5,30.9,30.2,29.3,29.2,28.8$, 27.3, 27.2, 25.7, 25.5, 25.4, 22.5, 19.2, 17.9, 17.8, 13.9, 8.23, 7.1, -4.1, -4.2, -5.0, -5.3; IR (ATR, $\mathrm{cm}^{-1}$ ) 3319, 2939, 1721, 1662, 1531, 1453, 1350, 1087; HRMS (ESI) calcd for $\mathrm{C}_{64} \mathrm{H}_{82} \mathrm{~N}_{6} \mathrm{O}_{21} \mathrm{SiSNa}[\mathrm{M}+\mathrm{Na}]^{+}$1353.4915; found $\mathrm{m} / \mathrm{z}$ 1353.4930 .
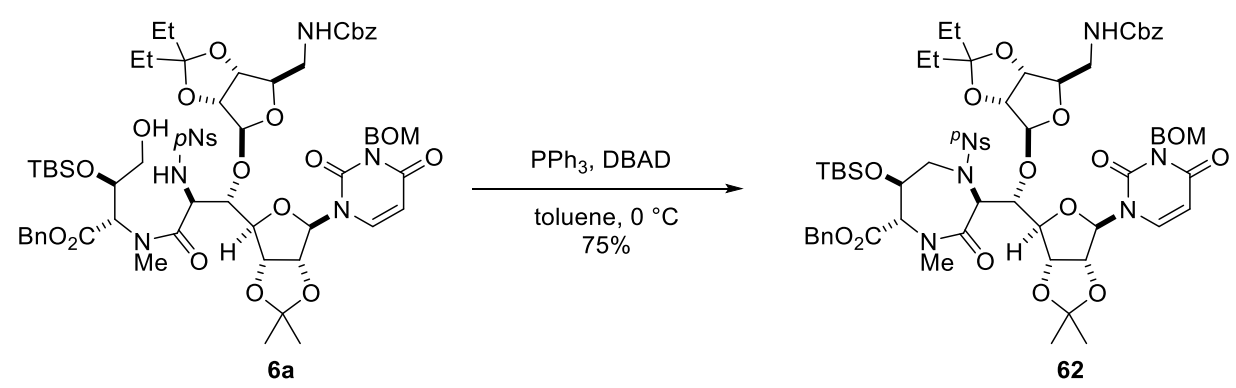

Compound 62: To a solution of 6a $(74.8 \mathrm{mg}, 56.2 \mu \mathrm{mol})$ in toluene $(8.6 \mathrm{~mL})$ were added $\mathrm{PPh}_{3}(119 \mathrm{mg}, 454 \mu \mathrm{mol})$ and DBAD $(88.7 \mathrm{mg}, 385 \mu \mathrm{mol})$ at $0{ }^{\circ} \mathrm{C}$, and the resultant solution was stirred at $0{ }^{\circ} \mathrm{C}$ for $14 \mathrm{~h}$. After the reaction mixture was concentrated under reduced pressure, purification by silica gel column chromatography (hexane/EtOAc $=1: 1)$ afforded $62(55.0 \mathrm{mg}, 75 \%)$ as a yellow amorphous: $[\alpha]^{25} \mathrm{D}+27.6^{\circ}\left(c 1.47, \mathrm{CHCl}_{3}\right) ;{ }^{1} \mathrm{H} \mathrm{NMR}\left(500 \mathrm{MHz}, \mathrm{CDCl}_{3}\right)$ $\delta 8.38(2 \mathrm{H}, \mathrm{d}, J=8.6 \mathrm{~Hz}), 8.07(2 \mathrm{H}, \mathrm{d}, J=9.2 \mathrm{~Hz}), 7.42-7.22(16 \mathrm{H}, \mathrm{m}), 5.70(1 \mathrm{H}, \mathrm{d}, J=9.2 \mathrm{~Hz}), 5.58-5.56(1 \mathrm{H}, \mathrm{br})$, $5.47(1 \mathrm{H}, \mathrm{d}, J=10.1 \mathrm{~Hz}), 5.43(1 \mathrm{H}, \mathrm{d}, J=10.1 \mathrm{~Hz}), 5.28(1 \mathrm{H}, \mathrm{d}, J=12.6 \mathrm{~Hz}), 5.22(1 \mathrm{H}, \mathrm{d}, J=12.6 \mathrm{~Hz}), 5.13(1 \mathrm{H}, \mathrm{d}, J$ $=12.4 \mathrm{~Hz}), 5.06(1 \mathrm{H}, \mathrm{d}, J=12.4 \mathrm{~Hz}), 5.04-4.94(3 \mathrm{H}, \mathrm{m}), 4.78-4.62(4 \mathrm{H}, \mathrm{m}), 4.44-3.98(6 \mathrm{H}, \mathrm{br} \mathrm{m}), 3.57(1 \mathrm{H}, \mathrm{dd}, J=$ 14.8, 6.4 Hz), 3.37-3.27 (1H, m), 3.24-2.98 (5H, m), $1.52(3 \mathrm{H}, \mathrm{s}), 1.45-1.36(2 \mathrm{H}, \mathrm{m}), 1.35-1.20(5 \mathrm{H}, \mathrm{m}), 0.93-0.72$ $(15 \mathrm{H}, \mathrm{m}), 0.10(3 \mathrm{H}, \mathrm{s}), 0.09(3 \mathrm{H}, \mathrm{s}) ;{ }^{13} \mathrm{C}$ NMR $\left(126 \mathrm{MHz}, \mathrm{CDCl}_{3}\right) \delta 167.8,162.4,156.6,150.6,150.2,145.4,140.7$, 137.9, 136.6, 134.9, 132.9, 129.0-127.6 (12C), 124.9, 117.3, 115.0, 102.2, 85.8, 84.0, 81.6, 80.4, 72.5 (5C), 70.3 (3C), 68.5, 66.6, 62.7, 44.0, 41.1, 31.5, 29.2, 28.8, 27.2, 25.5 (3C), 22.0, 8.3, 7.4, -4.9, -5.1; IR (ATR, $\mathrm{cm}^{-1}$ ) 2977, 2937, $1720,1670,1532,1455,1351,1250,1159,1086$; HRMS (ESI) calcd for $\mathrm{C}_{64} \mathrm{H}_{80} \mathrm{~N}_{6} \mathrm{O}_{20} \mathrm{SiS}[\mathrm{M}+\mathrm{Na}]^{+} 1335.4810$; found $\mathrm{m} / \mathrm{z} 1335.4850$. 

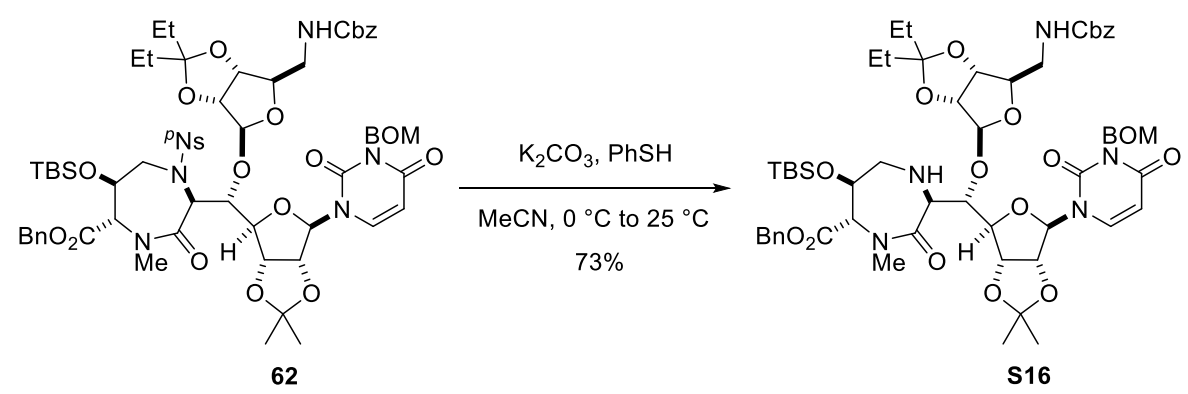

Compound S16: To a solution of $62(700 \mathrm{mg}, 533 \mu \mathrm{mol})$ in $\mathrm{MeCN}(18 \mathrm{~mL})$ were added $\mathrm{K}_{2} \mathrm{CO}_{3}(93.8 \mathrm{mg}, 679 \mu \mathrm{mol})$ and $\mathrm{PhSH}(171 \mu \mathrm{L}, 1.67 \mathrm{mmol})$ at $0{ }^{\circ} \mathrm{C}$, and the resultant solution was stirred at $25^{\circ} \mathrm{C}$ for $23 \mathrm{~h}$. The reaction was then quenched with $\mathrm{H}_{2} \mathrm{O}$. After extraction with $\mathrm{CHCl}_{3}$, the combined organic layers were washed with brine and dried over $\mathrm{Na}_{2} \mathrm{SO}_{4}$. After filtration and concentration under reduced pressure, purification by silica gel column chromatography (hexane/EtOAc $=2: 1)$ afforded S16 $(437 \mathrm{mg}, 73 \%)$ as a white amorphous: $[\alpha]^{25} \mathrm{D}+54.2^{\circ}\left(c 1.18, \mathrm{CHCl}_{3}\right)$; ${ }^{1} \mathrm{H}$ NMR $\left(500 \mathrm{MHz}, \mathrm{CDCl}_{3}\right) \delta$ 7.38-7.24 (15H, m), $7.19(1 \mathrm{H}, \mathrm{d}, J=8.0 \mathrm{~Hz}), 6.55(1 \mathrm{H}, \mathrm{m}), 5.69(1 \mathrm{H}, \mathrm{d}, J=8.0 \mathrm{~Hz}), 5.55(1 \mathrm{H}, \mathrm{d}$, $J=1.1 \mathrm{~Hz}), 5.48(1 \mathrm{H}, \mathrm{d}, J=9.7 \mathrm{~Hz}), 5.44(1 \mathrm{H}, \mathrm{d}, J=9.7 \mathrm{~Hz}), 5.35(1 \mathrm{H}, \mathrm{s}), 5.20(1 \mathrm{H}, \mathrm{d}, J=12.3 \mathrm{~Hz}), 5.13(1 \mathrm{H}, \mathrm{d}, J=$ $12.3 \mathrm{~Hz}), 5.06(2 \mathrm{H}, \mathrm{s}), 4.73(1 \mathrm{H}, \mathrm{d}, J=5.2 \mathrm{~Hz}), 4.70(2 \mathrm{H}, \mathrm{s}), 4.62(1 \mathrm{H}, \mathrm{d}, J=6.0 \mathrm{~Hz}), 4.48-4.36(3 \mathrm{H}, \mathrm{m}), 4.34-4.22$ $(3 \mathrm{H}, \mathrm{m}), 4.05(1 \mathrm{H}, \mathrm{d}, J=5.7 \mathrm{~Hz}), 3.54-3.49(1 \mathrm{H}, \mathrm{m}), 3.21(1 \mathrm{H}, \mathrm{s}), 3.00(1 \mathrm{H}, \mathrm{dd}, J=14.7,2.4 \mathrm{~Hz}), 2.97(3 \mathrm{H}, \mathrm{s}), 2.90$ $(1 \mathrm{H}, \mathrm{m}), 2.84(1 \mathrm{H}, \mathrm{d}, J=14.7 \mathrm{~Hz}), 1.51(3 \mathrm{H}, \mathrm{m}), 1.46(2 \mathrm{H}, \mathrm{m}), 1.32-1.22(5 \mathrm{H}, \mathrm{m}), 0.84(9 \mathrm{H}, \mathrm{s}), 0.77(6 \mathrm{H}, \mathrm{t}, J=7.5$ $\mathrm{Hz}), 0.11-0.01(6 \mathrm{H}, \mathrm{m}) ;{ }^{13} \mathrm{C} \mathrm{NMR}\left(126 \mathrm{MHz}, \mathrm{CDCl}_{3}\right) \delta 174.12,168.76,162.56,156.68,150.65,140.58,138.04$, 137.04, 134.88, 128.8-127.6 (9C) , 116.48 , 114.82 , 110.94 , 102.08 , 93.75 , 86.9, 86.6, 84.7, 82.1, 80.8, 79.1, 72.4, 70.3, 68.2, 67.5, 66.43 , 60.0, 52.3, 43.9, 39.7, 31.5, 29.6, 28.8, 27.3, 25.8, 25.4 (3C), 22.6, 17.7, 8.42, 7.35 , -4.72 , -4.91; IR (ATR, $\left.\mathrm{cm}^{-1}\right) 3339,2936,1721,1673,1450,1250,1091,742$; HRMS (ESI) calcd for $\mathrm{C}_{58} \mathrm{H}_{78} \mathrm{~N}_{5} \mathrm{O}_{16} \mathrm{Si}[\mathrm{M}+\mathrm{H}]^{+}$ 1128.5207 ; found $m / z 1128.5227$.
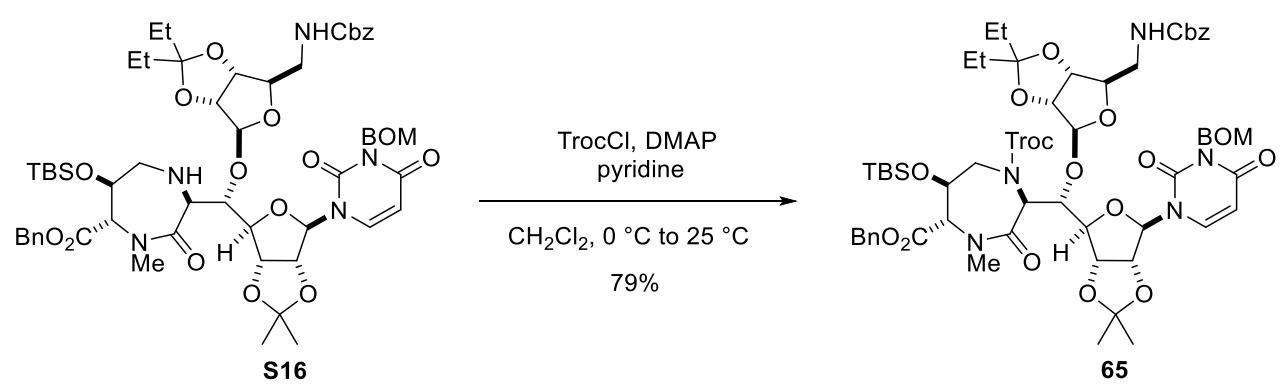

Compound 65: To a solution of $\mathbf{S 1 6}(425 \mathrm{mg}, 0.377 \mathrm{mmol})$ in $\mathrm{CH}_{2} \mathrm{Cl}_{2}(10 \mathrm{~mL})$ were added pyridine $(0.600 \mathrm{~mL}, 7.45$ mmol), DMAP (306 mg, $2.50 \mathrm{mmol})$ and $\operatorname{TrocCl}(0.800 \mathrm{~mL}, 5.93 \mathrm{mmol})$ at $0{ }^{\circ} \mathrm{C}$ and the resultant solution was stirred at $25{ }^{\circ} \mathrm{C}$ for $1.5 \mathrm{~h}$. The reaction was then quenched with $1.0 \mathrm{M}$ aqueous $\mathrm{HCl}$. The mixture was extracted with $\mathrm{CHCl}_{3}$, the organic layers were washed with brine and dried over $\mathrm{Na}_{2} \mathrm{SO}_{4}$. After filtration and concentrated under reduced pressure, purification by silica gel column chromatography (hexane/EtOAc $=1: 1)$ afforded $\mathbf{6 5}(387 \mathrm{mg}, 79 \%)$ as a white amorphous: $[\alpha]^{25} \mathrm{D}+25.8^{\circ}\left(c\right.$ 1.80, $\left.\mathrm{CHCl}_{3}\right) ;{ }^{1} \mathrm{H}$ NMR $\left(500 \mathrm{MHz}, \mathrm{CD}_{3} \mathrm{OD}\right) \delta$ 7.44-7.22 (16H, m), 5.82-5.75 (1H, m), 5.75-5.69 (2H, m), $5.48(2 \mathrm{H}, \mathrm{d}, J=10.0 \mathrm{~Hz}), 5.45(2 \mathrm{H}, \mathrm{d}, J=10.0 \mathrm{~Hz}), 5.20-4.99(6 \mathrm{H}, \mathrm{m}), 4.96-4.89(2 \mathrm{H}, \mathrm{m})$, 4.80-4.78 (2H, br m), 4.72-4.68 (3H, m), 4.50-4.44 (2H, m), $4.40(1 \mathrm{H}, \mathrm{d}, J=5.4 \mathrm{~Hz}), 4.28(1 \mathrm{H}, \mathrm{dd}, J=7.3,4.2 \mathrm{~Hz})$, $4.20(1 \mathrm{H}, \mathrm{d}, J=11.5 \mathrm{~Hz}), 4.02-3.94(2 \mathrm{H}, \mathrm{m}), 3.46-3.38(1 \mathrm{H}, \mathrm{m}), 3.23(1 \mathrm{H}, \mathrm{dd}, J=14.5,9.6 \mathrm{~Hz}), 3.14-3.01(4 \mathrm{H}, \mathrm{m})$, 1.69-1.51 (5H, m), $1.46(2 \mathrm{H}, \mathrm{q}, J=7.4 \mathrm{~Hz}), 1.33(3 \mathrm{H}, \mathrm{s}), 0.96-0.73(15 \mathrm{H}, \mathrm{m}), 0.10(3 \mathrm{H}, \mathrm{s}), 0.07(3 \mathrm{H}, \mathrm{s}) ;{ }^{13} \mathrm{C}$ NMR 
(126 MHz, $\left.\mathrm{CD}_{3} \mathrm{OD}\right) \delta 169.1,167.2,162.4,156.3,153.2,150.8,140.1,137.9,136.4,134.4,129.16-127.28$ (9C), 117.2, 114.6, 112.0, 102.0, 94.6, 93.6, 86.3, 86.1, 85.6, 83.7, 81.6, 81.2, 75.9, 72.2, 71.5, 70.3, 70.1, 67.9, 66.7, 62.0, 47.9, 44.0, 41.0, 29.3, 28.9, 27.1, 25.6 (3C), 25.3, 17.9, 8.3, 7.6, 7.4, -4.9, -5.2; IR (ATR, $\mathrm{cm}^{-1}$ ) 3353, 2936, 1721, 1669, $1458,1254,1091$; HRMS (ESI) calcd for $\mathrm{C}_{61} \mathrm{H}_{79} \mathrm{~N}_{5} \mathrm{O}_{18} \mathrm{SiCl}_{3} \mathrm{Na}[\mathrm{M}+\mathrm{Na}]^{+} 1325.4147$; found $m / z$ 1325.4156.
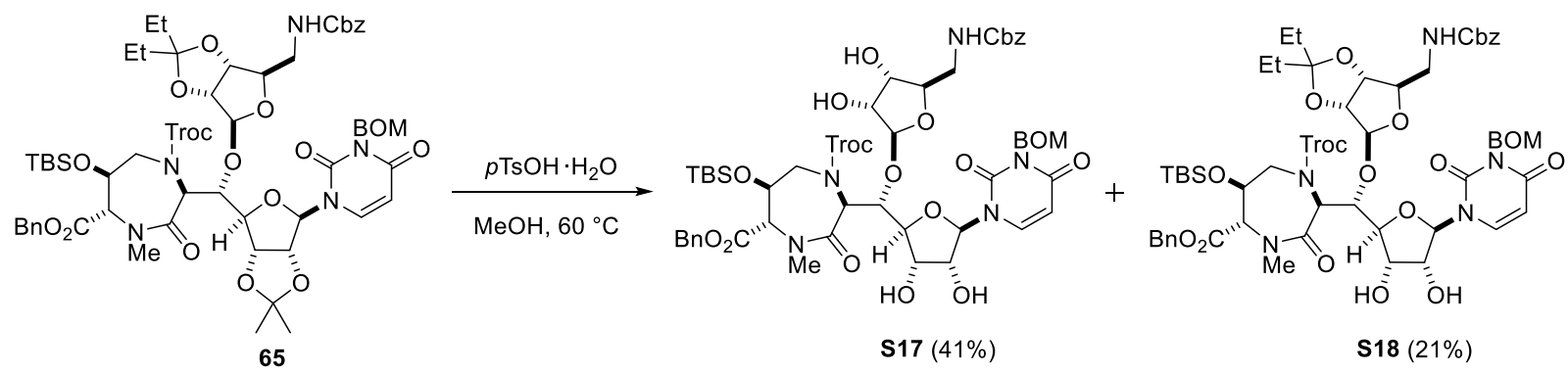

Compound S17: To a solution of 65 in $\mathrm{MeOH}(14 \mathrm{~mL})$ was added $p$ TsOH$\cdot \mathrm{H}_{2} \mathrm{O}(191 \mathrm{mg}, 1.00 \mathrm{mmol})$ at $25^{\circ} \mathrm{C}$, and the resultant solution was stirred at $50^{\circ} \mathrm{C}$ for $2 \mathrm{~h}$. The reaction was quenched with pyridine $(18.0 \mathrm{ml}, 223 \mathrm{mmol})$. After concentration under reduced pressure, purification by silica gel column chromatography (EtOAc) afforded compound S17 (138 mg, 41\%) as a white amorphous and compound S18 (76.8 $\mathrm{mg}, 21 \%)$ as a white amorphous: Compound S17: $[\alpha]^{25} \mathrm{D}+23.1^{\circ}(c 2.3, \mathrm{MeOH}) ;{ }^{1} \mathrm{H} \mathrm{NMR}\left(500 \mathrm{MHz}, \mathrm{CD}_{3} \mathrm{OD}\right) \delta 7.93(1 \mathrm{H}, \mathrm{d}, J=7.9 \mathrm{~Hz}), 7.40-7.24(15 \mathrm{H}, \mathrm{m}), 6.68(1 \mathrm{H}$, $\mathrm{m}), 5.86-5.75(2 \mathrm{H}, \mathrm{m}), 5.46-5.40(2 \mathrm{H}, \mathrm{m}), 5.16-5.00(6 \mathrm{H}, \mathrm{m}), 4.71(1 \mathrm{H}, \mathrm{d}, J=11.7 \mathrm{~Hz}), 4.66(2 \mathrm{H}, \mathrm{m}), 4.39(1 \mathrm{H}, \mathrm{s})$, $4.22(1 \mathrm{H}, \mathrm{d}, J=3.7 \mathrm{~Hz}), 4.16-4.00(2 \mathrm{H}, \mathrm{m}), 4.20-3.86(6 \mathrm{H}, \mathrm{m}), 3.67(1 \mathrm{H}, \mathrm{dd}, J=14.0,10.0 \mathrm{~Hz}), 3.45-3.38(1 \mathrm{H}, \mathrm{m})$, 3.28-3.24 (1H, m), 3.15-3.05 (4H, m), $0.90(9 \mathrm{H}, \mathrm{m}), 0.15(3 \mathrm{H}, \mathrm{s}), 0.13(3 \mathrm{H}, \mathrm{s}) ;{ }^{13} \mathrm{C}$ NMR $\left(126 \mathrm{MHz}, \mathrm{CD}_{3} \mathrm{OD}\right) \delta 171.8$, 169.0, 164.7, 158.4, 154.7, 152.4, 141.1, 139.3, 138.1, 136.3, 129.7-128.6 (9C), 112.5, 102.1, 96.0, 91.4 , 87.4, 83.6, 82.0, 77.1, 76.8, 75.2, 73.2, 73.1 (2C), 72.5, 71.54, 71.48, 69.1, 67.7, 64.9, 48.7, 45.3, 41.4, 26.4 (3C), 19.1, -4.59, -4.65; IR (ATR, $\mathrm{cm}^{-1}$ ) 3385, 2943, 1715, 1664, 1455, 1262, 1075, 752; HRMS (ESI) calcd for $\mathrm{C}_{53} \mathrm{H}_{67} \mathrm{~N}_{5} \mathrm{O}_{18} \mathrm{SiCl}_{3} \mathrm{Na}$ $[\mathrm{M}+\mathrm{Na}]^{+}$1217.3208; found $\mathrm{m} / \mathrm{z}$ 1217.3185. Compound S18: $[\alpha]^{25} \mathrm{D}+30.3^{\circ}\left(c 1.46, \mathrm{CHCl}_{3}\right) ;{ }^{1} \mathrm{H}$ NMR $(500 \mathrm{MHz}$, $\left.\mathrm{CDCl}_{3}\right) \delta 7.43-7.21(16 \mathrm{H}, \mathrm{m}), 5.79(1 \mathrm{H}, \mathrm{d}, J=6.0 \mathrm{~Hz}), 5.74(1 \mathrm{H}, \mathrm{d}, J=8.3 \mathrm{~Hz}), 5.47(1 \mathrm{H}, \mathrm{d}, J=9.7 \mathrm{~Hz}), 5.44(1 \mathrm{H}, \mathrm{d}$, $J=9.7 \mathrm{~Hz}), 5.33(1 \mathrm{H}, \mathrm{s}), 5.16-4.98(5 \mathrm{H}, \mathrm{m}), 4.83(1 \mathrm{H}, \mathrm{d}, J=8.9 \mathrm{~Hz}), 4.73-4.65(4 \mathrm{H}, \mathrm{m}), 4.55(1 \mathrm{H}, \mathrm{d}, J=6.3 \mathrm{~Hz}), 4.43$ $(1 \mathrm{H}, \mathrm{d}, J=6.3 \mathrm{~Hz}), 4.30(1 \mathrm{H}, \mathrm{dd}, J=7.7,4.0 \mathrm{~Hz}), 4.14(2 \mathrm{H}, \mathrm{dd}, J=12.0,4.3 \mathrm{~Hz}), 4.02(2 \mathrm{H}, \mathrm{dd}, J=14.5,5.6 \mathrm{~Hz}), 3.93$ $(2 \mathrm{H}, \mathrm{d}, J=7.4 \mathrm{~Hz}), 3.50-3.41(2 \mathrm{H}, \mathrm{m}), 3.22(1 \mathrm{H}, \mathrm{dd}, J=14.6,9.7 \mathrm{~Hz}), 3.13(3 \mathrm{H}, \mathrm{s}), 2.98-2.89(1 \mathrm{H}, \mathrm{m}), 1.61-1.52(2 \mathrm{H}$, m), $1.43(2 \mathrm{H}, \mathrm{q}, J=7.4 \mathrm{~Hz}), 0.87(9 \mathrm{H}, \mathrm{s}), 0.80(3 \mathrm{H}, \mathrm{t}, J=7.3 \mathrm{~Hz}), 0.75(3 \mathrm{H}, \mathrm{t}, J=7.4 \mathrm{~Hz}), 0.11(3 \mathrm{H}, \mathrm{s}), 0.08(3 \mathrm{H}, \mathrm{s})$; ${ }^{13} \mathrm{C}$ NMR $\left(126 \mathrm{MHz}, \mathrm{CDCl}_{3}\right) \delta{ }^{13} \mathrm{C}-\mathrm{NMR}\left(\mathrm{CDCl}_{3}\right) \delta: 168.0,167.1,162.4,156.2,154.3,151.4,139.0,137.8,136.4$, 134.2, 129.08-127.5 (9C), 116.9, 112.1, 102.2, 94.2, 90.5, 86.3, 85.4, 81.7, 80.4, 75.9, 73.5, 72.2, 70.8, 70.3, 69.9, 68.1, 66.7, 61.9, 48.6, 44.0, 41.1, 29.4, 28.8, 25.5 (3C), 17.8, 8.3, 7.3, -4.0, -5.1; IR (ATR, $\mathrm{cm}^{-1}$ ) 3366, 2955, 1714, 1661, 1456, 1255, 1090; HRMS (ESI) calcd for $\mathrm{C}_{58} \mathrm{H}_{75} \mathrm{~N}_{5} \mathrm{O}_{18} \mathrm{SiCl}_{3} \mathrm{Na}[\mathrm{M}+\mathrm{Na}]^{+}$1285.3834; found $m / z$ 1285.3821. 

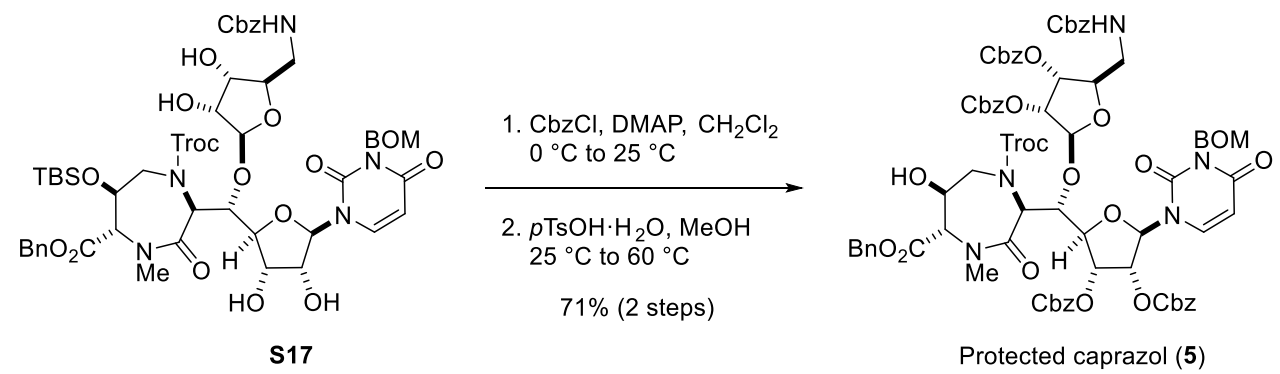

Compound 5: To a solution of $\mathbf{S 1 7}(138 \mathrm{mg}, 115 \mu \mathrm{mol})$ in $\mathrm{CH}_{2} \mathrm{Cl}_{2}(4.0 \mathrm{~mL})$ were added DMAP $(281 \mathrm{mg}, 2.30 \mathrm{mmol})$ and $\mathrm{CbzCl}(200 \mu \mathrm{L}, 1.40 \mathrm{mmol})$ at $0{ }^{\circ} \mathrm{C}$, and the resultant solution was stirred for $1 \mathrm{~h}$. The reaction was then quenched with $1.0 \mathrm{M}$ aqueous $\mathrm{HCl}$. After the mixture was extracted with $\mathrm{CHCl}_{3}$, the combined organic layers were washed with brine and dried over $\mathrm{Na}_{2} \mathrm{SO}_{4}$. Filtration and concentration under reduced pressure afforded a crude tetraol, which was used for the next reaction without further purification.

To a solution of the above tetraol in $\mathrm{MeOH}(15 \mathrm{~mL})$ was added $p \mathrm{TsOH} \cdot \mathrm{H}_{2} \mathrm{O}(355 \mathrm{mg}, 1.87 \mathrm{mmol})$ at $25^{\circ} \mathrm{C}$, and the resultant solution was stirred at $60{ }^{\circ} \mathrm{C}$ for $13 \mathrm{~h}$. The reaction was then quenched with saturated aqueous $\mathrm{NaHCO}_{3}$, and the mixture was extracted with $\mathrm{CHCl}_{3}$. The combined organic layers were washed with brine and dried over $\mathrm{Na}_{2} \mathrm{SO}_{4}$. After filtration and concentration under reduced pressure, purification by silica gel column chromatography (hexane/EtOAc $=1: 1)$ afforded a protected caprazol $5\left(132 \mathrm{mg}, 71 \%\right.$ for two steps) as a white amorphous: $[\alpha]^{25} \mathrm{D}$ $+23.6^{\circ}\left(c 1.68, \mathrm{CHCl}_{3}\right){ }^{1} \mathrm{H}$ NMR $\left(500 \mathrm{MHz}, \mathrm{CDCl}_{3}\right.$ at $60{ }^{\circ} \mathrm{C}$; mixture of rotamers) $\delta 7.51-7.10(36 \mathrm{H}, \mathrm{m}), 6.10(1 \mathrm{H}, \mathrm{br}$ s), 5.96-5.90 $(1 \mathrm{H}, \mathrm{m}), 5.80(1 \mathrm{H}, \mathrm{m}), 5.76(1 \mathrm{H}, \mathrm{d}, J=8.1 \mathrm{~Hz}), 5.47-5.24(5 \mathrm{H}, \mathrm{m}), 5.18-4.86(16 \mathrm{H}, \mathrm{m}), 4.71(1 \mathrm{H}, \mathrm{d}, J=$ $11.7 \mathrm{~Hz}), 4.65(2 \mathrm{H}, \mathrm{s}), 4.34-4.27(1 \mathrm{H}, \mathrm{m}), 4.20-4.04(3 \mathrm{H}, \mathrm{m}), 3.75-3.44(2 \mathrm{H}, \mathrm{m}), 3.35(1 \mathrm{H}, \mathrm{m}), 3.19-3.05(3 \mathrm{H}, \mathrm{m}) ;{ }^{13} \mathrm{C}$ $\mathrm{NMR}\left(126 \mathrm{MHz}, \mathrm{CDCl}_{3}\right.$ at $60^{\circ} \mathrm{C}$; mixture of rotamers) $\delta 169.3,169.2,168.6,168.0,162.6,157.2,154.5,154.4,154.3$, 154.2, 153.9, 153.6, 151.3, 151.2, 138.8, 138.4, 135.2, 135.2, 135.1, 129.1, 129.1, 128.9, 128.9, 128.7, 128.6, 128.3, 128.1, 128.0, 127.9, 109.0, 108.4, 103.3, 95.2, 94.7, 89.1, 88.6, 83.3, 83.0, 80.7, 79.6, 79.4, 78.3, 76.9, 76.3, 76.1, 75.4, 74.1, 73.9, 72.6, 71.5, 71.1, 70.3, 68.3, 67.6, 63.5, 47.8, 47.6, 43.4, 43.0, 41.4, 41.3; IR (ATR, cm $\left.{ }^{-1}\right)$ 3361, 3034, 2954, 1754, 1721, 1669, 1456, 1265, 746; HRMS (ESI) calcd for $\mathrm{C}_{79} \mathrm{H}_{76} \mathrm{~N}_{5} \mathrm{O}_{26} \mathrm{Cl}_{3}[\mathrm{M}+\mathrm{Na}]^{+} 1638.3736$; found $\mathrm{m} / \mathrm{z}$ 1638.3698.
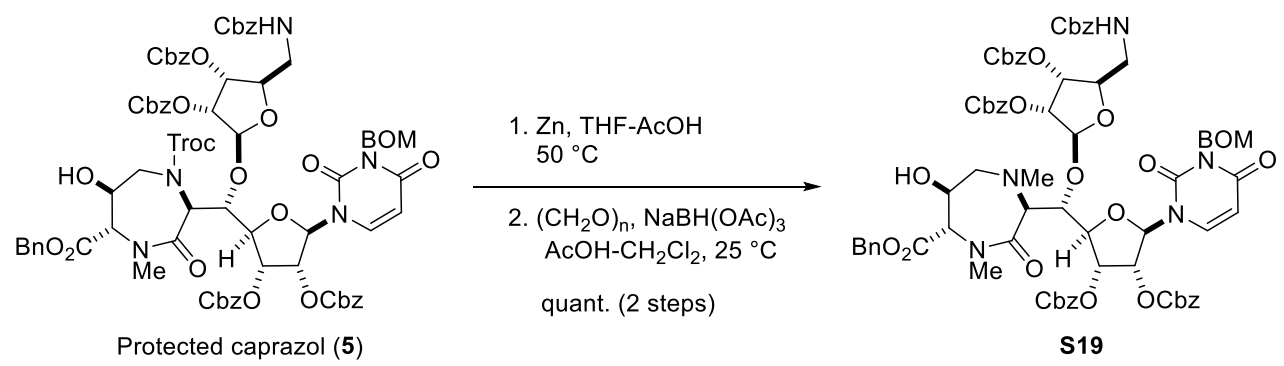

Compound S19: To a solution of $5(13.2 \mathrm{mg}, 8.16 \mu \mathrm{mol})$ in THF-AcOH $(2.2 \mathrm{~mL}, \mathrm{v} / \mathrm{v}=10: 1)$ was added $\mathrm{Zn}$ powder (213 mg, $3.26 \mathrm{mmol}$ ) at $25^{\circ} \mathrm{C}$ and the resultant solution was then stirred at $50{ }^{\circ} \mathrm{C}$ for $2.5 \mathrm{~h}$. After filtration with celite, concentration under reduced pressure afforded a crude amine, which was used for the next reaction without further purification.

To a solution of the above crude amine in $\mathrm{CH}_{2} \mathrm{Cl}_{2}(2.0 \mathrm{~mL})$ were added paraformaldehyde $(24.3 \mathrm{mg}), \mathrm{AcOH}(100 \mu \mathrm{L}$, $1.75 \mathrm{mmol})$ and $\mathrm{NaBH}(\mathrm{OAc})_{3}(43.1 \mathrm{mg}, 203 \mu \mathrm{mol})$ at $25^{\circ} \mathrm{C}$, and the resultant solution was stirred at $25^{\circ} \mathrm{C}$ for $11 \mathrm{~h}$. The reaction was then quenched with saturated aqueous $\mathrm{NaHCO}_{3}$. After extraction with $\mathrm{CHCl}_{3}$, the combined organic 
layers were washed with brine and dried over $\mathrm{Na}_{2} \mathrm{SO}_{4}$. After filtration and concentration under reduced pressure, purification by silica gel column chromatography (hexane/EtOAc $=1: 1)$ afforded $\mathbf{S 1 9}$ (11.9 $\mathrm{mg}$, quant., 2 steps) as a white amorphous; $[\alpha]^{25} \mathrm{D}-0.1^{\circ}\left(c \quad 0.91, \mathrm{CHCl}_{3}\right) ;{ }^{1} \mathrm{H} \mathrm{NMR}\left(500 \mathrm{MHz}, \mathrm{CDCl}_{3}\right) \delta 7.35(1 \mathrm{H}, \mathrm{d}, J=8.3 \mathrm{~Hz}), 7.31-7.00$ $(35 \mathrm{H}, \mathrm{m}), 6.46-6.29(1 \mathrm{H}, \mathrm{m}), 5.81(1 \mathrm{H}, \mathrm{d}, J=4.3 \mathrm{~Hz}), 5.73(1 \mathrm{H}, \mathrm{br} \mathrm{m}), 5.35-5.25(3 \mathrm{H}, \mathrm{m}), 5.23(1 \mathrm{H}, \mathrm{dd}, J=5.2,5.2$ $\mathrm{Hz}), 5.19(1 \mathrm{H}, \mathrm{d}, J=4.6 \mathrm{~Hz}), 5.14(1 \mathrm{H}, \mathrm{br} \mathrm{s}), 5.11-4.78(14 \mathrm{H}, \mathrm{m}), 4.55(2 \mathrm{H}, \mathrm{s}), 4.38(2 \mathrm{H}, \mathrm{d}, J=6.0 \mathrm{~Hz}), 4.24-4.17(2 \mathrm{H}$, $\mathrm{m}), 4.01(1 \mathrm{H}, \mathrm{d}, J=4.6 \mathrm{~Hz}), 3.62-3.47(2 \mathrm{H}, \mathrm{m}), 3.32(1 \mathrm{H}, \mathrm{d}, J=14.3 \mathrm{~Hz}), 3.04(3 \mathrm{H}, \mathrm{s}), 2.74(1 \mathrm{H}, \mathrm{d}, J=12.0 \mathrm{~Hz}), 2.29$ $(3 \mathrm{H}, \mathrm{s}) ;{ }^{13} \mathrm{C}$ NMR $\left(126 \mathrm{MHz}, \mathrm{CDCl}_{3}\right) \delta 168.5,162.2,154.2,154.1,154.0,154.0,151.0,138.19,138.15,138.1,136.8$, 135.1, 135.0, 135.0, 134.9, 134.8, 129.2-127.3 (22H, m), 107.1, 103.2, 87.8, 81.7, 78.0, 78.6, 76.1, 73.7, 72.8, 72.27, 72.25, 72.2, 70.65-70.22 (5H, m), 69.9, 68.1, 67.5, 66.9, 59.3, 42.2, 38.4, 29.7; IR (ATR, cm ${ }^{-1}$ ) 3420, 2926, 1753, 1719, $1672,1455,1384,1269$; HRMS (ESI) calcd for $\mathrm{C}_{77} \mathrm{H}_{77} \mathrm{~N}_{5} \mathrm{O}_{24}[\mathrm{M}+\mathrm{Na}]^{+} 1478.4851$; found $m / z 1478.4821$.
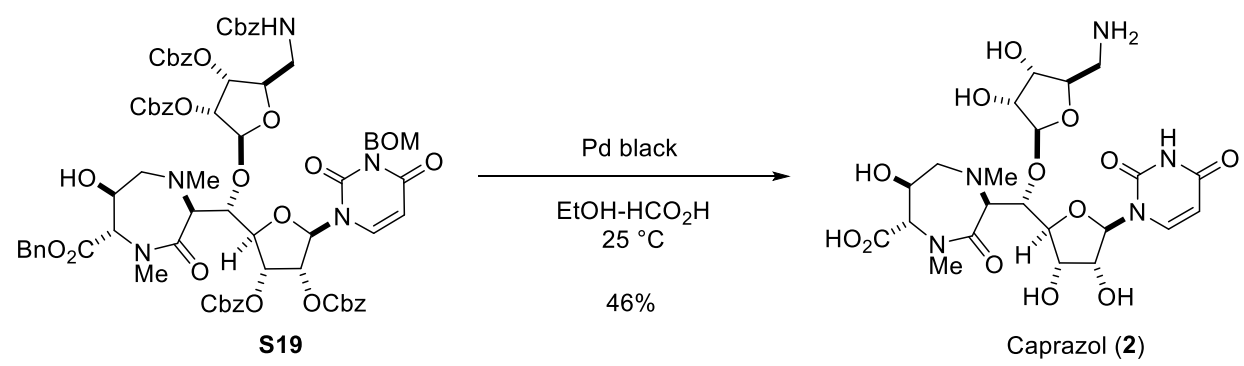

Caprazol (2): To a solution of $\mathbf{S 1 9}(3.3 \mathrm{mg}, 2.27 \mu \mathrm{mol})$ in $\mathrm{EtOH}-\mathrm{HCO}_{2} \mathrm{H}(1.1 \mathrm{~mL}, \mathrm{v} / \mathrm{v}=10: 1)$ was added Pd black (22.4 mg, $210 \mu \mathrm{mol}$ ) at $25^{\circ} \mathrm{C}$, and the resultant mixture was stirred at $25{ }^{\circ} \mathrm{C}$ for $4 \mathrm{~h}$. The reaction was then quenched with $\mathrm{pH} 7$ buffer. After filtration with celite, the filtrate was concentrated under reduced pressure. The resultant residue was purified by reversed phase HPLC column $(10 \times 250 \mathrm{~mm})$; mobile phase, $\mathrm{H}_{2} \mathrm{O}$; flow rate, $3.0 \mathrm{ml} /$ minute; detection, $\mathrm{UV} ; 258 \mathrm{~nm}$. The eluent was collected and concentrated under reduced pressure to give a caprazol (2) $(0.6 \mathrm{mg}, 46 \%)$ as a white solid: $[\alpha]^{27} \mathrm{D}+14.3^{\circ}\left(c 0.15\right.$, DMSO); ${ }^{1} \mathrm{H}$ NMR $\left(500 \mathrm{MHz}, \mathrm{D}_{2} \mathrm{O}\right) \delta 7.82(1 \mathrm{H}, \mathrm{d}, J=8.0 \mathrm{~Hz}), 5.87(1 \mathrm{H}, \mathrm{d}, J=8.0$ $\mathrm{Hz}), 5.65(1 \mathrm{H}, \mathrm{d}, J=1.7 \mathrm{~Hz}), 5.22(1 \mathrm{H}, \mathrm{s}), 4.51-4.47(1 \mathrm{H}, \mathrm{m}), 4.44(1 \mathrm{H}, \mathrm{d}, J=9.2 \mathrm{~Hz}), 4.35(1 \mathrm{H}, \mathrm{dd}, J=5.2,1.7 \mathrm{~Hz})$, $4.31(1 \mathrm{H}, \mathrm{dd}, J=6.6,4.9 \mathrm{~Hz}), 4.28-4.23(2 \mathrm{H}, \mathrm{m}), 4.20-4.16(2 \mathrm{H}, \mathrm{m}), 4.13(1 \mathrm{H}, \mathrm{dd}, J=8.0,5.2 \mathrm{~Hz}), 3.91(1 \mathrm{H}, \mathrm{d}, J=$ $9.2 \mathrm{~Hz}), 3.37(1 \mathrm{H}, \mathrm{dd}, J=13.7,3.4 \mathrm{~Hz}), 3.25(1 \mathrm{H}, \mathrm{dd}, J=14.0,4.3 \mathrm{~Hz}), 3.18(1 \mathrm{H}, \mathrm{d}, J=15.5 \mathrm{~Hz}), 3.12(3 \mathrm{H}, \mathrm{s}), 3.07$ $(1 \mathrm{H}, \mathrm{d}, J=14.9 \mathrm{~Hz}), 2.48(3 \mathrm{H}, \mathrm{s}) ;{ }^{13} \mathrm{C}$ NMR $\left(126 \mathrm{MHz}, \mathrm{CDCl}_{3}\right) \delta 176.6,175.1,169.7,154.3,145.3,113.7,104.1,94.3$, 84.8, 81.4, 80.0, 77.9, 76.5, 73.0, 72.4, 71.8, 71.7, 65.9, 61.5, 42.6, 41.6, 39.4; HRMS (ESI) calcd for $\mathrm{C}_{22} \mathrm{H}_{34} \mathrm{~N}_{5} \mathrm{O}_{13}$ $[\mathrm{M}+\mathrm{H}]^{+}$576.2148; found $\mathrm{m} / \mathrm{z} 576.2161$. 


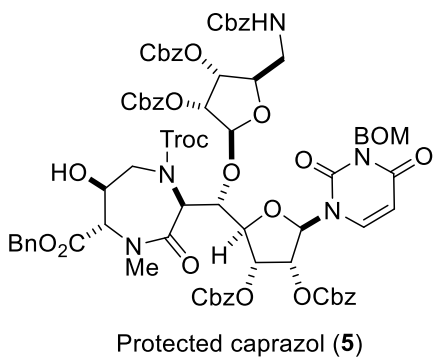

Protected caprazol (5)
1. 55b, EDCI, DMAP, $\mathrm{CH}_{2} \mathrm{Cl}_{2}$

$0{ }^{\circ} \mathrm{C}$ to $25^{\circ} \mathrm{C}$

2. $\mathrm{Zn}, \mathrm{AcOH} / \mathrm{THF}, 25^{\circ} \mathrm{C}$

3. $\mathrm{AcOH}, \mathrm{DCE}, 25^{\circ} \mathrm{C}$

4. $\left(\mathrm{CH}_{2} \mathrm{O}\right)_{n}, \mathrm{NaBH}(\mathrm{OAc})_{3}$ $\mathrm{AcOH} / \mathrm{DCE}, 25^{\circ} \mathrm{C}$

5. HF.py, THF, $0^{\circ} \mathrm{C}$ to $25^{\circ} \mathrm{C}$

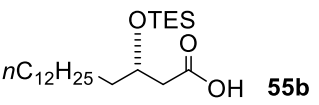

$43 \%$ (5 steps)

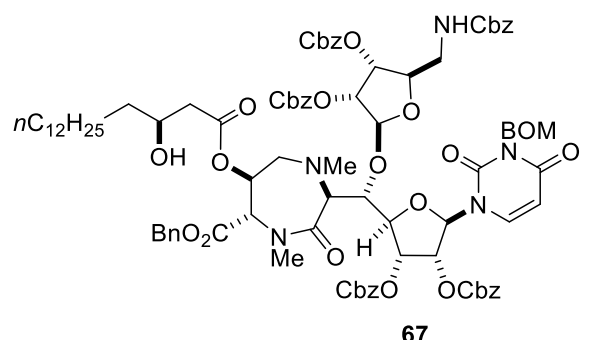

67

Compound 67: To a solution of 5 (40.7 mg, $25.2 \mu \mathrm{mol})$ and carboxylic acid $\mathbf{5 5 b}(48.5 \mathrm{mg}, 125 \mu \mathrm{mol})$ in $\mathrm{CH}_{2} \mathrm{Cl}_{2}(12$ $\mathrm{mL})$ were added DMAP $(20.6 \mathrm{mg}, 169 \mu \mathrm{mol})$ and EDCI $(24.4 \mathrm{mg}, 127 \mu \mathrm{mol})$ at $0{ }^{\circ} \mathrm{C}$, and the resultant solution was stirred at $25{ }^{\circ} \mathrm{C}$ for $2 \mathrm{~h}$. The reaction was then quenched with saturated aqueous $\mathrm{NH}_{4} \mathrm{Cl}$. The mixture was extracted with $\mathrm{CHCl}_{3}$, and the combined organic layers were washed with brine and dried over $\mathrm{Na}_{2} \mathrm{SO}_{4}$. Filtration and concentration under reduced pressure afforded a crude coupling product which was used for the next reaction without further purification. (The acylated product was unstable under the basic conditions.)

To a solution of the above coupling product in THF $(10 \mathrm{~mL})$ were added $\mathrm{Zn}$ powder $(308 \mathrm{mg}, 4.71 \mathrm{mmol})$ and $\mathrm{AcOH}$ $(130 \mu \mathrm{L}, 2.27 \mathrm{mmol})$ at $25^{\circ} \mathrm{C}$, and the resultant solution was stirred at $50{ }^{\circ} \mathrm{C}$ for $2.5 \mathrm{~h}$. The reaction was then quenched with saturated aqueous $\mathrm{NaHCO}_{3}$. After extraction with $\mathrm{CHCl}_{3}$, the combined organic layers were washed with brine and dried over $\mathrm{Na}_{2} \mathrm{SO}_{4}$. Filtration and concentration under reduced pressure afforded a crude product which was used for the next reaction without further purification.

The above crude product was dissolved in dichloroethane $(4.0 \mathrm{~mL})$, and $\mathrm{AcOH}(50 \mu \mathrm{L})$ was added at $25{ }^{\circ} \mathrm{C}$. The resultant solution was stirred at $25^{\circ} \mathrm{C}$ for $2 \mathrm{~h}$. The reaction was then quenched with saturated aqueous $\mathrm{NaHCO}_{3}$. After extraction with $\mathrm{CHCl}_{3}$, the combined organic layers were washed with brine and dried over $\mathrm{Na}_{2} \mathrm{SO}_{4}$. After filtration and concentration under reduced pressure, purification by flash silica gel column chromatography (hexane/EtOAc $=$ 2:1) afforded a crude secondary amine $\mathbf{6 6}$ which was used for the next reaction without further purification.

To a solution of the above secondary amine 66 in dichloroethane $(4.0 \mathrm{~mL})$ were added paraformaldehyde $(59.8 \mathrm{mg})$, $\mathrm{AcOH}(100 \mu \mathrm{L}, 1.75 \mathrm{mmol})$ and $\mathrm{NaBH}(\mathrm{OAc})_{3}(45.7 \mathrm{mg}, 216 \mu \mathrm{mol})$ at $25^{\circ} \mathrm{C}$, and the resultant solution was stirred at $25{ }^{\circ} \mathrm{C}$ for $6 \mathrm{~h}$. The reaction was then quenched with saturated aqueous $\mathrm{NaHCO}_{3}$. After extraction with $\mathrm{CHCl}_{3}$, the combined organic layers were washed with brine and dried over $\mathrm{Na}_{2} \mathrm{SO}_{4}$. Filtration and concentration under reduced pressure afforded a crude methylated product which was used for the next reaction without further purification.

To a solution of the above methylated product in THF $(4.0 \mathrm{~mL})$ was added HF.pyr $(90.0 \mu \mathrm{L}, 999 \mu \mathrm{mol})$ at $0{ }^{\circ} \mathrm{C}$, and the resultant solution was stirred at $25^{\circ} \mathrm{C}$ for $2 \mathrm{~h}$. The reaction was then quenched with saturated aqueous $\mathrm{NaHCO}_{3}$. After extraction with $\mathrm{CHCl}_{3}$, the combined organic layers were washed with brine and dried over $\mathrm{Na}_{2} \mathrm{SO}_{4}$. After filtration and concentration under reduced pressure, purification by flash silica gel column chromatography (hexane/EtOAc $=2: 1)$ afforded $67\left(23.6 \mathrm{mg}, 43 \%\right.$ for five steps) as a white amorphous: $[\alpha]^{25} \mathrm{D}-3.0^{\circ}\left(c 1.55, \mathrm{CHCl}_{3}\right)$; ${ }^{1} \mathrm{H}$ NMR $\left(500 \mathrm{MHz}, \mathrm{CDCl}_{3}\right.$ at $\left.60{ }^{\circ} \mathrm{C}\right) \delta 7.42(1 \mathrm{H}, \mathrm{d}, J=8.3 \mathrm{~Hz}), 7.34-7.19(35 \mathrm{H}, \mathrm{m}), 6.14(1 \mathrm{H}, \mathrm{br} \mathrm{s}), 5.88(1 \mathrm{H}, \mathrm{d}, J=$ $4.6 \mathrm{~Hz}), 5.83-5.76(1 \mathrm{H}, \mathrm{m}), 5.40-5.35(2 \mathrm{H}, \mathrm{m}), 5.31(1 \mathrm{H}, \mathrm{dd}, J=5.9,4.7 \mathrm{~Hz}), 5.28(1 \mathrm{H}, \mathrm{d}, J=5.2 \mathrm{~Hz}), 5.21(1 \mathrm{H}, \mathrm{d}, J=$ $7.2 \mathrm{~Hz}), 5.18-5.02(14 \mathrm{H}, \mathrm{m}), 4.90(1 \mathrm{H}, \mathrm{d}, J=8.3 \mathrm{~Hz}), 4.62(2 \mathrm{H}, \mathrm{s}), 4.49-4.44(2 \mathrm{H}, \mathrm{m}), 4.37(1 \mathrm{H}, \mathrm{d}, J=4.3 \mathrm{~Hz})$, 4.33-4.27 (1H, m), $4.01(1 \mathrm{H}, \mathrm{m}), 3.71-3.57(2 \mathrm{H}, \mathrm{m}), 3.45-3.36(1 \mathrm{H}, \mathrm{d}, J=13.7 \mathrm{~Hz}), 3.11-3.00(4 \mathrm{H}, \mathrm{m}), 2.47(1 \mathrm{H}, \mathrm{dd}, J$ $=16.1,3.1 \mathrm{~Hz}), 2.42-2.33(4 \mathrm{H}, \mathrm{m}), 1.55-1.42(2 \mathrm{H}, \mathrm{m}), 1.35-1.24(22 \mathrm{H}, \mathrm{m}), 0.88(3 \mathrm{H}, \mathrm{t}, J=7.0 \mathrm{~Hz}) ;{ }^{13} \mathrm{C} \mathrm{NMR}(100$ $\left.\mathrm{MHz}, \mathrm{CDCl}_{3}\right) \delta 173.0,171.8,170.9,167.7,162.3,156.9,154.2,154.1,154.0,150.9,138.1,138.0,136.5,134.9,134.9$, 134.8, 134.6, 134.6, 129.0-127.7 (22C), 107.3, 103.3, 87.5, 81.1, 79.8, 78.3, 76.1, 75.7, 73.5, 72.2, 72.1, 71.0, 
70.6-70.3 (5C), 68.2, 67.9, 67.0, 64.0, 63.00, 42.0, 41.8, 38.6, 36.89, 32.1, 29.8-29.3 (9C), 25.6, 22.8, 14.3; IR (ATR, $\left.\mathrm{cm}^{-1}\right) 2925,2855,1753,1721,1674,1455,1383,1265$; HRMS (ESI) calcd for $\mathrm{C}_{93} \mathrm{H}_{108} \mathrm{~N}_{5} \mathrm{O}_{26}[\mathrm{M}+\mathrm{H}]^{+} 1710.7277$; found $m / z 1710.7273$.

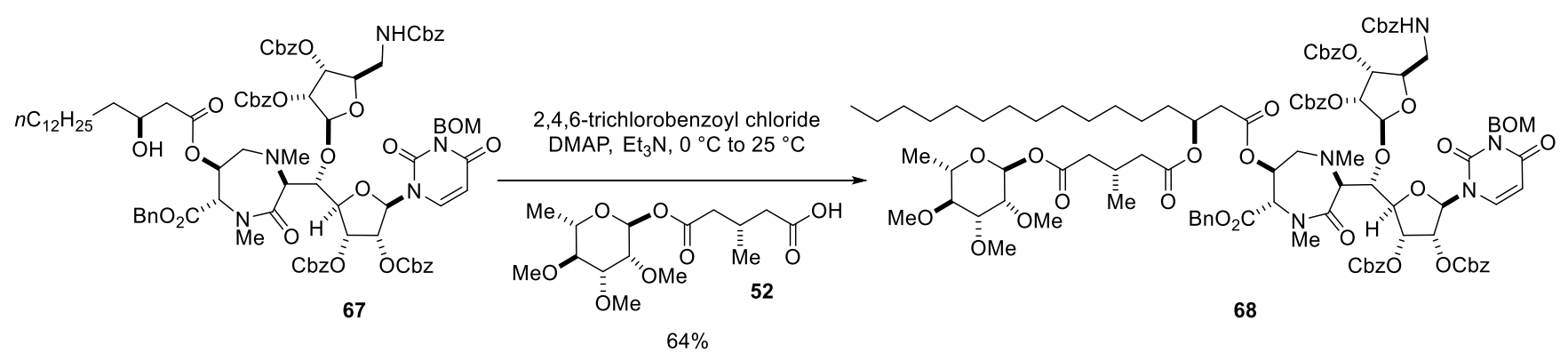

Protected caprazamycin (66): To a solution of carboxylic acid $67(39.1 \mathrm{mg}, 117 \mu \mathrm{mol})$ in $\mathrm{CH}_{2} \mathrm{Cl}_{2}(5.0 \mathrm{~mL})$ was added $\mathrm{Et}_{3} \mathrm{~N}(17.0 \mu \mathrm{L}, 121 \mu \mathrm{mol})$ and 2,4,6-trichlorobenzoyl chloride $(19.0 \mu \mathrm{mol}, 121 \mu \mathrm{mol})$ at $0{ }^{\circ} \mathrm{C}$, and the resultant solution was stirred at $25^{\circ} \mathrm{C}$ for $1.5 \mathrm{~h}$. The reaction mixture was added dropwise to a solution of $\mathbf{5 2}$ and DMAP (17.4 $\mathrm{mg}, 142 \mu \mathrm{mol})$ in $\mathrm{CH}_{2} \mathrm{Cl}_{2}(2.0 \mathrm{~mL})$ at $0{ }^{\circ} \mathrm{C}$. The resultant solution was then stirred at $25^{\circ} \mathrm{C}$ for $2.5 \mathrm{~h}$. The reaction was then quenched with $1.0 \mathrm{M}$ aqueous $\mathrm{HCl}$. After extraction with $\mathrm{CHCl}_{3}$, the combined organic layers were washed with saturated aqueous $\mathrm{NaHCO}_{3}$ and brine, and then dried over $\mathrm{Na}_{2} \mathrm{SO}_{4}$. After filtration and concentration under reduced pressure, purification by silica gel column chromatography (hexane/EtOAc $=3: 2$ ) afforded protected caprazamycin A (68) $(14.1 \mathrm{mg}, 64 \%)$ as a white amorphous: $[\alpha]^{25} \mathrm{D}-10.5^{\circ}\left(c 0.50, \mathrm{CHCl}_{3}\right) ;{ }^{1} \mathrm{H} \mathrm{NMR}\left(500 \mathrm{MHz}, \mathrm{CDCl}_{3}\right.$; mixture of rotamers) $\delta 7.42(1 \mathrm{H}, \mathrm{d}, J=8.0 \mathrm{~Hz}), 7.39-7.15(35 \mathrm{H}, \mathrm{m}), 6.41-6.34(1 \mathrm{H}, \mathrm{br} \mathrm{m}), 6.15(1 \mathrm{H}, \mathrm{m}), 5.83-5.76(2 \mathrm{H}, \mathrm{m})$, 5.45-5.26 (6H, m), 5.22-4.97 (17H, m), $4.86(1 \mathrm{H}, \mathrm{d}, J=12.3 \mathrm{~Hz}), 4.71-4.57(2 \mathrm{H}, \mathrm{m}), 4.48-4.38(2 \mathrm{H}, \mathrm{m}), 4.36-4.21(1 \mathrm{H}$, $\mathrm{m}), 3.70-3.35(14 \mathrm{H}, \mathrm{m}), 3.17(1 \mathrm{H}, \mathrm{q}, J=9.0 \mathrm{~Hz}), 3.1-2.96(4 \mathrm{H}, \mathrm{m}), 2.61-2.15(10 \mathrm{H}, \mathrm{m}), 1.67-1.50(\mathrm{~m}, 2 \mathrm{H}), 1.37-1.17$ $(22 \mathrm{H}, \mathrm{m}), 1.00(3 \mathrm{H}, \mathrm{d}, J=6.5 \mathrm{~Hz}), 0.88(3 \mathrm{H}, \mathrm{t}, J=7.0 \mathrm{~Hz}) ;{ }^{13} \mathrm{C} \mathrm{NMR}\left(126 \mathrm{MHz}, \mathrm{CDCl}_{3} ;\right.$ mixture of rotamers) $\delta 171.4$, 170.9 , 170.4, 169.3, 167.7, 162.3, 156.9, 154.1, 154.0, 153.9, 153.8, 150.9, 137.9, 136.4, 134.74, 134.70, 134.6, 134.9, 134.4, 129.0-127.6 (24 C), 107.5, 103.3, 90.9, 87.8, 81.7, 81.2, 80.9, 80.0, 78.5, 76.2, 73.5, 72.2, 71.2, 70.6-70.2 (5C), $67.8,66.9,63.8,62.8,61.2,59.1,58.0,42.0,40.65,40.58,39.2,38.5,36.9,34.2,32.1,29.8-29.2$ (12C), 27.5, 25.3, 22.8, 19.8, 17.9, 14.3; IR (ATR, $\mathrm{cm}^{-1}$ ) 2929, 1751, 1680, 1454, 1273; HRMS (ESI) calcd for $\mathrm{C}_{108} \mathrm{H}_{131} \mathrm{~N}_{5} \mathrm{O}_{33} \mathrm{Na}$ $[\mathrm{M}+\mathrm{Na}]^{+}$2048.8619; found $\mathrm{m} / \mathrm{z} 2048.8539$.
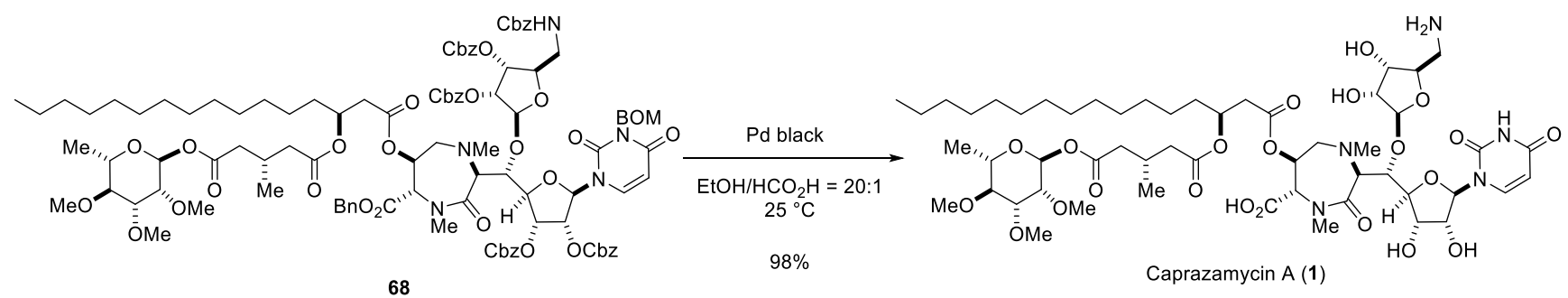

Caprazamycin A (1): To a solution of protected caprazamycin A (68) $(6.5 \mathrm{mg}, 3.21 \mu \mathrm{mol})$ in EtOH- $\mathrm{HCO}_{2} \mathrm{H}(1.9 \mathrm{ml}$, $\mathrm{v} / \mathrm{v}=20: 1)$ was added Pd black $(66.5 \mathrm{mg}, 625 \mu \mathrm{mol})$ at $25^{\circ} \mathrm{C}$, and the resultant mixture was stirred at $25^{\circ} \mathrm{C}$ for $1.5 \mathrm{~h}$. 
After filtration through celite, the filtrate was concentrated under reduced pressure. The resultant residue was purified by $\mathrm{C} 18$ reversed phase HPLC, COSMOSIL PACKED COLUMN (5C 18 -MS-II, $10 \times 250 \mathrm{~mm}$, nacalai tesque Co., Ltd., Japan); mobile phase, $75 \%$ aq. MeCN; flow rate, $3.0 \mathrm{ml} /$ minute; detection, UV; $258 \mathrm{~nm}$. Caprazamycin A was eluted at 11-18 $\mathrm{min}$ as an isolated peak. The eluent was collected and concentrated under reduced pressure to give a pure caprazamycin A (1) $(3.6 \mathrm{mg}, 98 \%)$ as a colorless powder: $[\alpha]^{25} \mathrm{D}-7.6^{\circ}\left(c \quad 0.54\right.$, DMSO); ${ }^{1} \mathrm{H}$ NMR (500 MHz, DMSO-d $\left.6: \mathrm{D}_{2} \mathrm{O}=20: 1\right) \delta 7.81(1 \mathrm{H}, \mathrm{d}, J=8.3 \mathrm{~Hz}), 5.94(1 \mathrm{H}, \mathrm{d}, J=2.0 \mathrm{~Hz}), 5.64(1 \mathrm{H}, \mathrm{d}, J=8.3 \mathrm{~Hz}), 5.55(1 \mathrm{H}, \mathrm{d}, J=$ $2.9 \mathrm{~Hz}), 5.37(1 \mathrm{H}, \mathrm{br} \mathrm{s}), 5.15-5.07(1 \mathrm{H}, \mathrm{m}), 5.00(1 \mathrm{H}, \mathrm{s}), 4.16(1 \mathrm{H}, \mathrm{d}, J=7.0 \mathrm{~Hz}), 4.09(1 \mathrm{H}, \mathrm{m}), 4.02-3.68(6 \mathrm{H}, \mathrm{m})$, $3.61(1 \mathrm{H}, \mathrm{dd}, J=2.7,2.7 \mathrm{~Hz}), 3.55-3.45(2 \mathrm{H}, \mathrm{m}), 3.40(3 \mathrm{H}, \mathrm{s}), 3.39-3.32(7 \mathrm{H}, \mathrm{m}), 3.06-2.85(8 \mathrm{H}, \mathrm{m}), 2.67-2.21(6 \mathrm{H}$, m), $2.16(1 \mathrm{H}, \mathrm{dd}, J=14.8,7.5 \mathrm{~Hz}), 1.60-1.49(2 \mathrm{H}, \mathrm{m}), 1.29-1.16(22 \mathrm{H}, \mathrm{m}), 1.13(3 \mathrm{H}, \mathrm{d}, J=6.3 \mathrm{~Hz}), 0.91(3 \mathrm{H}, \mathrm{d}, J=$ $6.3 \mathrm{~Hz}), 0.84(3 \mathrm{H}, \mathrm{t}, J=6.9 \mathrm{~Hz}) ;{ }^{1} \mathrm{H}$ NMR $\left(500 \mathrm{MHz}, \mathrm{DMSO}_{6} \mathrm{~d}_{6}: \mathrm{D}_{2} \mathrm{O}: \mathrm{DCO}_{2} \mathrm{D}=20: 1: 1\right) \delta 7.70(1 \mathrm{H}, \mathrm{d}, J=8.0 \mathrm{~Hz})$, $5.93(1 \mathrm{H}, \mathrm{d}, J=2.0 \mathrm{~Hz}), 5.66(1 \mathrm{H}, \mathrm{d}, J=8.0 \mathrm{~Hz}), 5.49(1 \mathrm{H}, \mathrm{d}, J=1.7 \mathrm{~Hz}), 5.25(1 \mathrm{H}, \mathrm{br} \mathrm{s}), 5.15-5.07(1 \mathrm{H}, \mathrm{m}), 5.00$ $(1 \mathrm{H}, \mathrm{s}), 4.60(1 \mathrm{H}, \mathrm{d}, J=4.0 \mathrm{~Hz}), 4.14(1 \mathrm{H}, \mathrm{d}, J=9.2 \mathrm{~Hz}), 4.08(1 \mathrm{H}, \mathrm{d}, J=8.3 \mathrm{~Hz}), 4.02-3.94(2 \mathrm{H}, \mathrm{m}), 3.92(1 \mathrm{H}, \mathrm{dd}, J$ $=4.6,1.7 \mathrm{~Hz}), 3.86(1 \mathrm{H}, \mathrm{d}, J=2.0 \mathrm{~Hz}), 3.81(1 \mathrm{H}, \mathrm{dd}, J=7.7,4.9 \mathrm{~Hz}), 3.59(1 \mathrm{H}, \mathrm{dd}, J=3.0,2.1 \mathrm{~Hz}), 3.56-3.45(2 \mathrm{H}$, m), $3.39(3 \mathrm{H}, \mathrm{s}), 3.38-3.31(7 \mathrm{H}, \mathrm{m}), 3.22(1 \mathrm{H}, \mathrm{d}, J=14.6 \mathrm{~Hz}), 3.16-3.06(1 \mathrm{H}, \mathrm{m}), 3.04-2.96(5 \mathrm{H}, \mathrm{m}), 2.66-2.60(2 \mathrm{H}$, m), 2.41-2.21 (4H, m), $2.16(1 \mathrm{H}, \mathrm{dd}, J=14.7,7.3 \mathrm{~Hz}), 1.58-1.51(2 \mathrm{H}, \mathrm{m}), 1.27-1.15(22 \mathrm{H}, \mathrm{m}), 1.13(3 \mathrm{H}, \mathrm{d}, J=6.3 \mathrm{~Hz})$, $0.91(3 \mathrm{H}, \mathrm{d}, J=6.0 \mathrm{~Hz}), 0.82(3 \mathrm{H}, \mathrm{t}, J=6.9 \mathrm{~Hz}) ;{ }^{13} \mathrm{C}$ NMR $\left(126 \mathrm{MHz}, \mathrm{DMSO}-\mathrm{d}_{6}: \mathrm{D}_{2} \mathrm{O}: \mathrm{DCO}_{2} \mathrm{D}=20: 1: 1\right) \delta 171.6$, $170.7,170.5,170.1,169.5,163.9,150.6,140.2,110.4,101.3,90.8,89.8,82.4,81.2,80.4,78.4,75.5$ (2C), 74.7, 74.5, 70.9, 70.4, 70.2, 70.1, 69.9 (2C), 69.1, 63.3, 60.5, 58.7, 57.0, 40.3-38.8 (4C), 37.7, 36.1, 33.6, 31.6, .5-28.8 (8C), 27.5, 24.9, 22.4, 19.4, 18.0, 14.3; IR (ATR, cm ${ }^{-1}$ ) 3192, 2925, 2858, 1734, 1684, 1653, 1456, 1213, 1029; HRMS (ESI) calcd for $\mathrm{C}_{53} \mathrm{H}_{86} \mathrm{~N}_{5} \mathrm{O}_{22}[\mathrm{M}-\mathrm{H}]^{-} 1144.5770$; found $\mathrm{m} / \mathrm{z} 1144.5717$. 


\section{Computational study of thiourea catalyzed aldol reaction using benzaldehyde and catalyst 14a using}

\section{Gaussian 09}

The calculation of transition states A-C were first performed using density functional theory (DFT) methods at the B3LYP/6-31G(d) level. In order to gain the effect of dispersion energies, the calculation of transition states A-C were also performed using DFT methods at the $6-31 \mathrm{G}(\mathrm{d}, \mathrm{p})$ level with several different functionals, such as M062X ${ }^{(10)}$ functional, as well as $\omega \mathrm{B} 97 \mathrm{xD}^{(11)}$ and $\mathrm{APFD}^{(12)}$ functionals including dispersion. Once the stationary points were obtained, the harmonic vibrational frequencies were calculated at the same level to estimate the Gibbs free energy. All of the Gibbs free energy values reported in this paper were calculated for a temperature of $298.15 \mathrm{~K}$. In addition, intrinsic reaction coordinate (IRC) ${ }^{(13)}$ calculations were performed at the same level to connect all the transition states with the corresponding reactants and products. Single point energy (SPE) calculations was also performed at the $6-311+\mathrm{G}(\mathrm{d}, \mathrm{p})$ level to evaluate the basis set effect, and a self-consistent reaction field (SCRF) method based on the polarizable continuum model (PCM) $)^{(14)}$ was used to simulate the solvent effect for toluene.

(A)

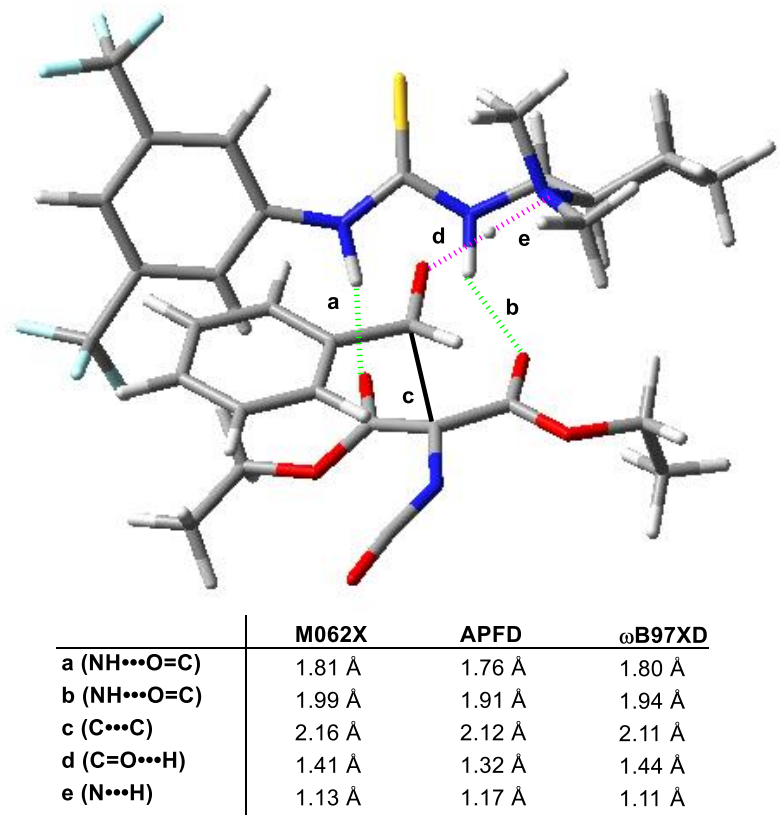

(B)

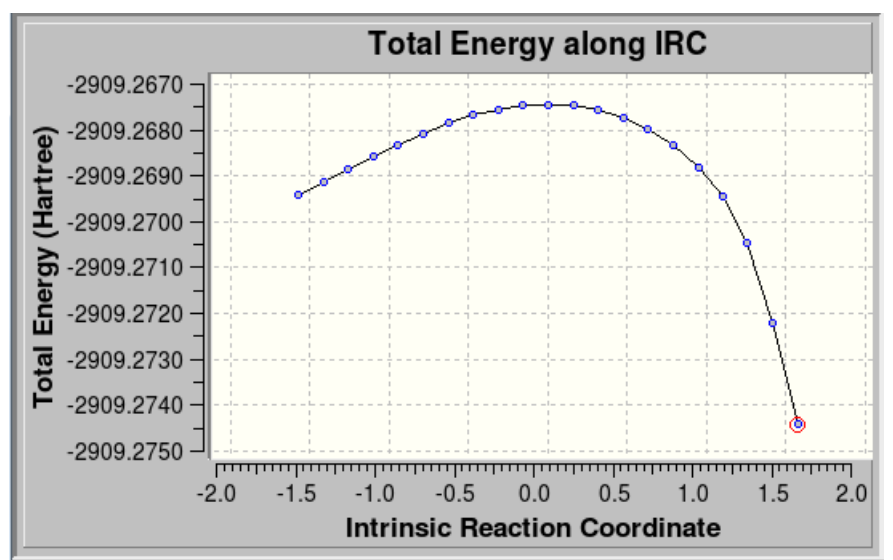

(C)
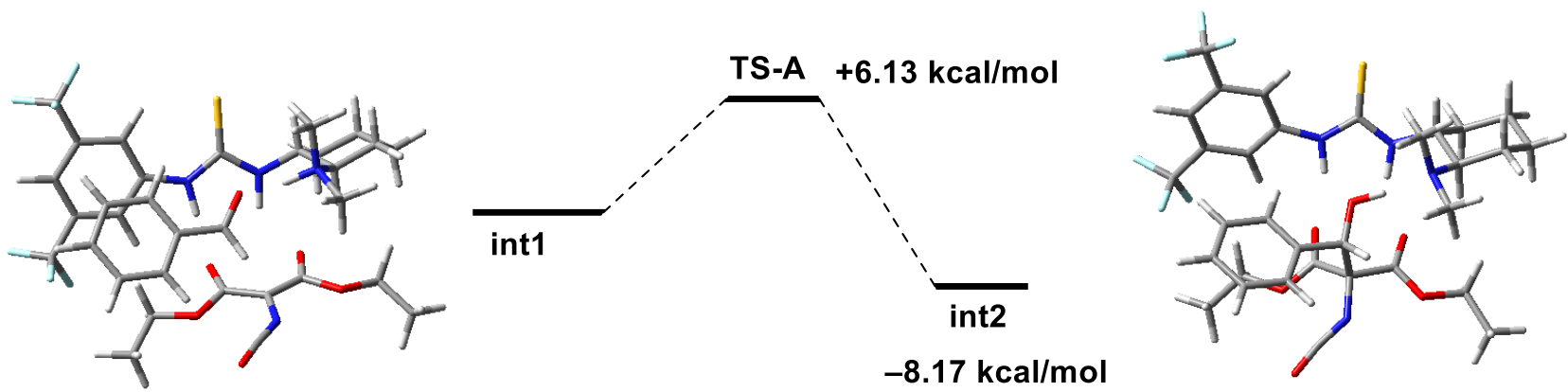

Figure S1. (A) Structure of TS-A (B) IRC calculations for TS-A $(\omega \mathrm{B} 97 \mathrm{xD} / 6-31 \mathrm{G}(\mathrm{d}, \mathrm{p})$ level) (C) Energy diagram for TS-A $(\omega \mathrm{B} 97 \mathrm{xD} / 6-31 \mathrm{G}(\mathrm{d}, \mathrm{p})$ level $)$ 
(A)

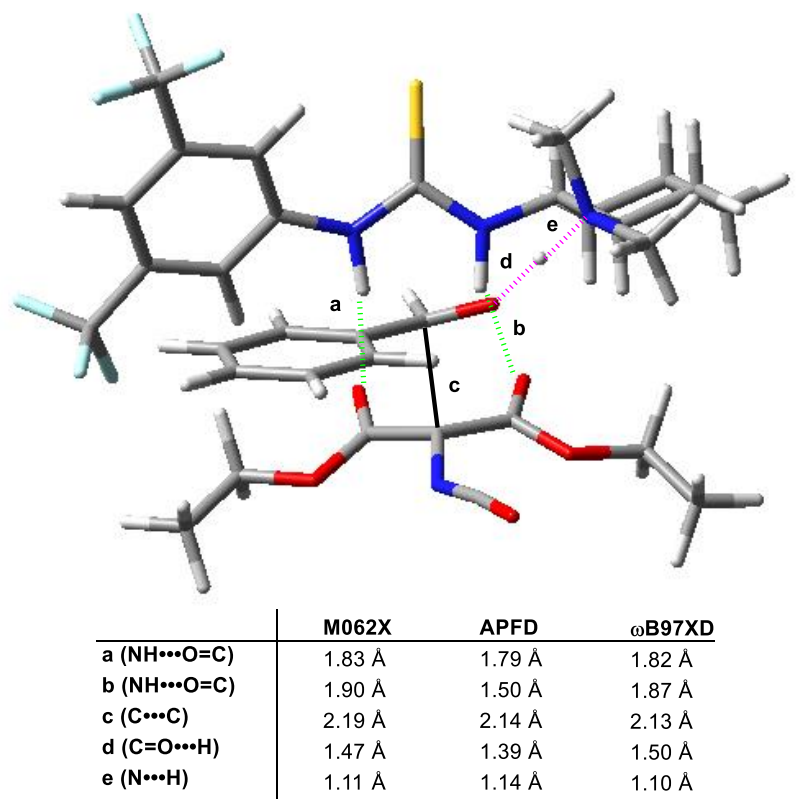

(B)

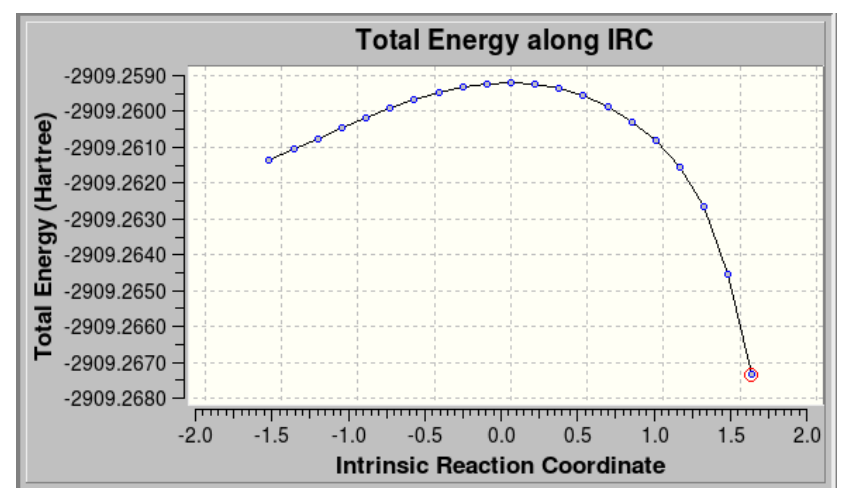

Figure S2. (A) Structure of TS-B (B) IRC calculations for TS-B $(\omega B$ B $7 x D / 6-31 G(d, p)$ level)

(A)

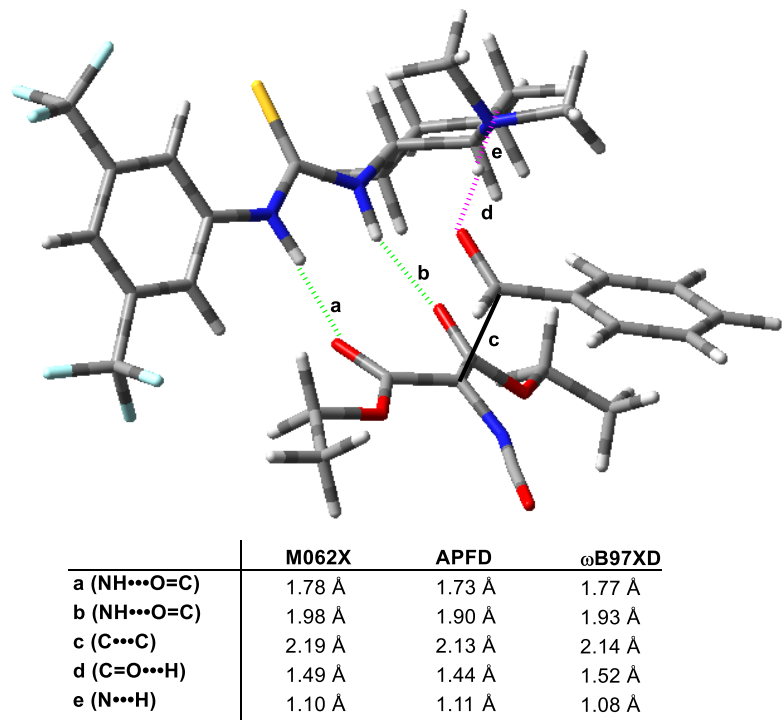

(B)

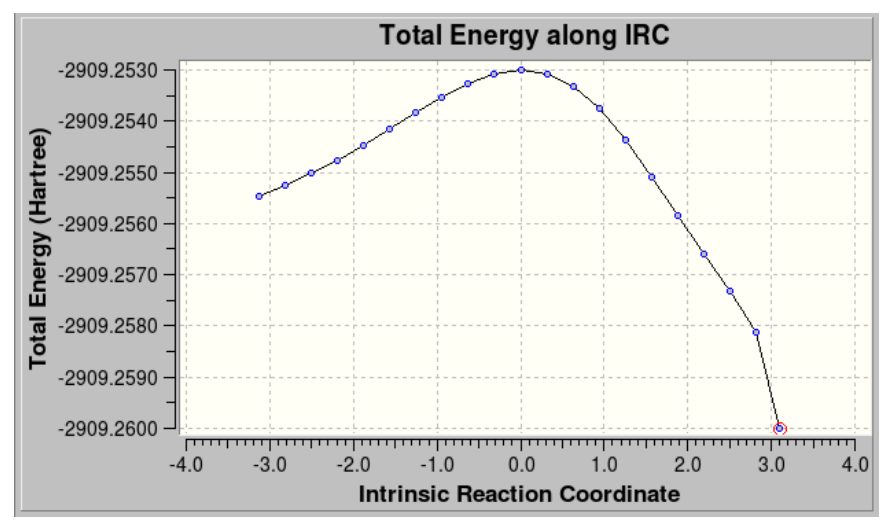

Figure S3. (A) Structure of TS-C (B) IRC calculations for TS-C ( $\omega B$ 97xD/6-31G(d,p) level) 


\begin{tabular}{|l|l|l|}
\hline & $\Delta \mathbf{G}^{\ddagger}\left(\Delta \mathbf{G}_{\text {TS-B-B }}-\Delta \mathbf{G}_{\text {TS-A }}\right)$ & $\Delta \mathbf{G}^{\ddagger}\left(\Delta \mathbf{G}_{\text {TS-C }}-\Delta \mathbf{G}_{\text {TS-A }}\right)$ \\
\hline B3LYP/6-311+G(d,p)//B3LYP/6-31G(d) & $+1.95 \mathrm{kcal} / \mathrm{mol}$ & $+4.42 \mathrm{kcal} / \mathrm{mol}$ \\
\hline M062X/6-311+G(d,p)//M062X/6-31G(d,p) & $+6.07 \mathrm{kcal} / \mathrm{mol}$ & $+7.29 \mathrm{kcal} / \mathrm{mol}$ \\
\hline APFD/6-311+G(d,p)//APFD/6-31G(d,p) & $+4.55 \mathrm{kcal} / \mathrm{mol}$ & $+6.97 \mathrm{kcal} / \mathrm{mol}$ \\
\hline$\omega \mathbf{B 9 7 X D / 6 - 3 1 1 + G ( d , p ) / / \omega B 9 7 X D ~ / 6 - 3 1 G ( d , p )}$ & $+4.68 \mathrm{kcal} / \mathrm{mol}$ & $+4.70 \mathrm{kcal} / \mathrm{mol}$ \\
\hline
\end{tabular}

In each case, the TS-A was suggested to be the favored transition state.

Figure S4. Comparison of the Gibbs free energies of the transition states

Cartesian coordinates and total energies for the proposed transition state structures

\section{TS-A (B3LYP)}

Zero-point correction=

Thermal correction to Energy=

Thermal correction to Enthalpy=

Thermal correction to Gibbs Free Energy=

Sum of electronic and zero-point Energies=

Sum of electronic and thermal Energies=

Sum of electronic and thermal Enthalpies=

Sum of electronic and thermal Free Energies=

$\mathrm{E}(\mathrm{RB} 3 \mathrm{LYP})=-2909.91949435$

Imaginary frequency $=248 \mathrm{i}$

0.685455 (Hartree/Particle)
0.734772
0.735716
0.596351
-2909.234039
-2909.184722
-2909.183778
-2909.323144

0.685455 (Hartree/Particle)

0.735716

0.596351

2909.234039

$-2909.183778$

$-2909.323144$

Relative energy $(B 3 L Y P / 6-311+G(d, p))=-2910.68104472$

01

$\begin{array}{lrrr}\mathrm{C} & 4.94448200 & -1.39062500 & -0.33890400 \\ \mathrm{C} & 2.84320200 & 0.40739400 & -0.17601200 \\ \mathrm{C} & 3.63650700 & -1.85958100 & -0.43769000 \\ \mathrm{C} & 5.22463200 & -0.03519400 & -0.15114300 \\ \mathrm{C} & 4.15959600 & 0.85857200 & -0.08067300 \\ \mathrm{C} & 2.56535600 & -0.95674300 & -0.33530600 \\ \mathrm{H} & 3.44588900 & -2.91270300 & -0.57935600 \\ \mathrm{H} & 6.24725000 & 0.31163700 & -0.06579400 \\ \mathrm{H} & 2.02081600 & 1.11187600 & -0.12635300 \\ \mathrm{~N} & 1.21055300 & -1.31658000 & -0.44441800 \\ \mathrm{H} & 0.59696900 & -0.53630100 & -0.69148500 \\ \mathrm{C} & 0.56864500 & -2.46707200 & -0.06680200\end{array}$


H

$\mathrm{H}$

$\mathrm{H}$

$\mathrm{H}$

$\mathrm{H}$

$\mathrm{H}$

$\mathrm{H}$

$\mathrm{H}$

H

C

O

O

C

$\mathrm{H}$

$\mathrm{H}$

C

$\mathrm{H}$

$\mathrm{H}$

$\mathrm{H}$

C

$\mathrm{N}$

C

O

C

H

$\mathrm{H}$

C

$\mathrm{H}$

$\mathrm{H}$

H $\begin{array}{lll}1.32058400 & -3.81397200 & 0.63957000\end{array}$

$\begin{array}{lll}-0.77183900 & -2.40457300 & -0.29543700\end{array}$

$\begin{array}{lll}-1.17135400 & -1.56333900 & -0.71812700\end{array}$

$\begin{array}{lll}-1.69470100 & -3.49350600 & -0.02860800\end{array}$

$\begin{array}{lll}-3.84159400 & -4.22717900 & 1.07061300\end{array}$

$\begin{array}{lll}-3.00923600 & -5.43424300 & -1.00838000\end{array}$

$\begin{array}{lll}-4.23814800 & -5.00477000 & -0.19773200\end{array}$

$-2.09908200 \quad-4.23815800 \quad-1.31794500$

$\begin{array}{lll}-2.96788300 & -3.01561300 & 0.70581700\end{array}$

$\begin{array}{lll}-3.29270400 & -4.89259000 & 1.75055800\end{array}$

$\begin{array}{lll}-2.44069500 & -6.18300700 & -0.43904800\end{array}$

$\begin{array}{lll}-4.88194300 & -4.36654100 & -0.82000000\end{array}$

$\begin{array}{lll}-2.60154800 & -3.53598000 & -1.99698300\end{array}$

$\begin{array}{lll}-3.52462800 & -2.36085600 & 0.02761800\end{array}$

$\begin{array}{lll}-1.15296500 & -4.20196800 & 0.60462200\end{array}$

$\begin{array}{lll}-4.75026500 & -3.90952300 & 1.59397400\end{array}$

$\begin{array}{lll}-3.32202100 & -5.92174400 & -1.93940900\end{array}$

$\begin{array}{lll}-4.84074200 & -5.87636400 & 0.08385600\end{array}$

$\begin{array}{lll}-1.18137800 & -4.56977700 & -1.81480200\end{array}$

$\begin{array}{lll}-1.13287400 & 1.86177000 & -1.25907400\end{array}$

$\begin{array}{lll}-0.33705700 & 0.94330500 & -1.40195400\end{array}$

$\begin{array}{lll}-0.83177100 & 3.13871900 & -1.54442500\end{array}$

$\begin{array}{lll}0.50918200 & 3.42584800 & -2.01356600\end{array}$

$1.20105100 \quad 3.30079000 \quad-1.17691700$

$\begin{array}{lll}0.77178200 & 2.69980800 & -2.78788200\end{array}$

$\begin{array}{lll}0.50914300 & 4.85155800 & -2.53088100\end{array}$

$\begin{array}{lll}0.20479400 & 5.55056100 & -1.74609500\end{array}$

$\begin{array}{lll}-0.17677300 & 4.96303200 & -3.37661300\end{array}$

$\begin{array}{lll}1.51757400 & 5.11981700 & -2.86392500\end{array}$

$\begin{array}{lll}-2.50820600 & 1.72893700 & -0.69667700\end{array}$

$\begin{array}{lll}-3.30364300 & 2.89745500 & -0.69163800\end{array}$

$\begin{array}{lll}-3.26051900 & 0.53354600 & -1.15741700\end{array}$

$\begin{array}{lll}-4.58566600 & 0.77462900 & -1.26529800\end{array}$

$\begin{array}{lll}-5.40871900 & -0.31402500 & -1.72922700\end{array}$

$\begin{array}{lll}-4.95121700 & -0.75496900 & -2.62004900\end{array}$

$\begin{array}{lll}-5.44557500 & -1.09266300 & -0.95705800\end{array}$

$\begin{array}{lll}-6.78802700 & 0.25210200 & -2.01324600\end{array}$

$\begin{array}{lll}-6.73694200 & 1.02449500 & -2.78657100\end{array}$

$\begin{array}{lll}-7.22216600 & 0.69737200 & -1.11253000\end{array}$

$\begin{array}{lll}-7.45373700 & -0.54487500 & -2.36235600\end{array}$ 


\begin{tabular}{|c|c|c|c|}
\hline $\mathrm{O}$ & -2.78433100 & -0.57772600 & -1.35854100 \\
\hline $\mathrm{C}$ & -3.16747300 & 4.09354500 & -0.79813000 \\
\hline $\mathrm{O}$ & -3.20560600 & 5.26998600 & -0.88329700 \\
\hline $\mathrm{N}$ & -2.64811600 & -2.11098200 & 1.88225800 \\
\hline $\mathrm{H}$ & -2.18007100 & -1.14315000 & 1.48551900 \\
\hline $\mathrm{C}$ & -1.68218000 & -2.66175000 & 2.87561400 \\
\hline $\mathrm{H}$ & -1.45609700 & -1.86778200 & 3.59037300 \\
\hline $\mathrm{H}$ & -0.75901000 & -2.96163100 & 2.37877600 \\
\hline $\mathrm{H}$ & -2.12058200 & -3.51557700 & 3.39971700 \\
\hline $\mathrm{C}$ & -3.87243800 & -1.62088300 & 2.57270100 \\
\hline $\mathrm{H}$ & -3.58251400 & -0.79286700 & 3.22280200 \\
\hline $\mathrm{H}$ & -4.33136700 & -2.41036100 & 3.17174400 \\
\hline $\mathrm{H}$ & -4.58530800 & -1.25965100 & 1.82803600 \\
\hline $\mathrm{C}$ & 6.09731400 & -2.34783400 & -0.50049300 \\
\hline $\mathrm{C}$ & 4.40371900 & 2.32136000 & 0.16748300 \\
\hline $\mathrm{F}$ & 6.63672400 & -2.26661800 & -1.74068800 \\
\hline $\mathrm{F}$ & 5.73202100 & -3.63058300 & -0.30963600 \\
\hline $\mathrm{F}$ & 7.09443300 & -2.07221500 & 0.37215800 \\
\hline $\mathrm{F}$ & 5.68532900 & 2.67509800 & -0.05033300 \\
\hline $\mathrm{F}$ & 4.10891000 & 2.66953300 & 1.44667600 \\
\hline $\mathrm{F}$ & 3.62969400 & 3.10325300 & -0.62688100 \\
\hline $\mathrm{C}$ & -2.16121100 & 1.23948200 & 1.19183700 \\
\hline $\mathrm{H}$ & -3.23597900 & 1.28806700 & 1.44518900 \\
\hline $\mathrm{O}$ & -1.56080200 & 0.09033600 & 1.25951500 \\
\hline $\mathrm{C}$ & -1.40540600 & 2.42635400 & 1.72459900 \\
\hline $\mathrm{C}$ & 0.04971000 & 4.61503200 & 2.70963900 \\
\hline $\mathrm{C}$ & -2.06619400 & 3.59013800 & 2.13857000 \\
\hline $\mathrm{C}$ & -0.00951800 & 2.35913500 & 1.84261900 \\
\hline $\mathrm{C}$ & 0.71575600 & 3.44784600 & 2.32236900 \\
\hline $\mathrm{C}$ & -1.34197000 & 4.67983200 & 2.62649600 \\
\hline $\mathrm{H}$ & -3.15053700 & 3.64080800 & 2.08722900 \\
\hline $\mathrm{H}$ & 0.48560500 & 1.43254600 & 1.57402800 \\
\hline $\mathrm{H}$ & 1.79819100 & 3.38476600 & 2.39038300 \\
\hline $\mathrm{H}$ & -1.86650400 & 5.57812400 & 2.94086100 \\
\hline $\mathrm{H}$ & 0.61334100 & 5.46499400 & 3.08523100 \\
\hline
\end{tabular}

\section{TS-A (M062X)}

Zero-point correction= 0.693272 (Hartree/Particle)

Thermal correction to Energy= 0.741095

Thermal correction to Enthalpy= 0.742040 
Thermal correction to Gibbs Free Energy=

Sum of electronic and zero-point Energies=

Sum of electronic and thermal Energies=

Sum of electronic and thermal Enthalpies=

Sum of electronic and thermal Free Energies=
0.609102

$-2908.299333$

$-2908.251510$

$-2908.250566$

$-2908.383503$

$\mathrm{E}(\mathrm{RM} 062 \mathrm{X})=-2908.99260538$

Imaginary frequency $=199 \mathrm{i}$

Relative energy $(\mathrm{M} 062 \mathrm{X} / 6-311+\mathrm{G}(\mathrm{d}, \mathrm{p}))=-2909.73321383$

01

$\mathrm{C}$

C

$\mathrm{C}$

C

C

C

$\mathrm{H}$

$\mathrm{H}$

$\mathrm{H}$

N

$\mathrm{H}$

C

$\mathrm{S}$

$\mathrm{N}$

$\mathrm{H}$

C

C

C

C

C

C

$\mathrm{H}$

$\mathrm{H}$

$\mathrm{H}$

$\mathrm{H}$

$\mathrm{H}$

$\mathrm{H}$

$\mathrm{H}$

\begin{tabular}{|c|c|c|}
\hline & -2.06870200 & \\
\hline & & \\
\hline & & \\
\hline & & -0.30337000 \\
\hline & & \\
\hline 2.06346300 & & \\
\hline 2.45841700 & -3.20567800 & \\
\hline & & \\
\hline & & \\
\hline & & \\
\hline 0.25143400 & -0.58485700 & \\
\hline & 100 & \\
\hline & & \\
\hline-1.48525400 & 92000 & \\
\hline-1.64 & 900 & -0. \\
\hline & & \\
\hline-4.89768200 & -3.23828100 & 0.4548430 \\
\hline-4.43060900 & -4.23373200 & -1.83 \\
\hline-5.49148000 & -3.636 & \\
\hline-3.23358100 & -3.29434100 & -1.99063600 \\
\hline-3.7484 & -2.25930300 & \\
\hline-4.53422700 & -4.13498100 & \\
\hline-4.08113700 & -5.18695100 & -1.41355100 \\
\hline-5.92491300 & -2.74360600 & -1.37295500 \\
\hline-3.52899700 & -2.37142900 & -2.5073960 \\
\hline-4.11703200 & -1.39144500 & -0.33086700 \\
\hline-2.33215800 & -3.85673500 & -0.1349120 \\
\hline 5.67931800 & -2.78952600 & 1.0768560 \\
\hline
\end{tabular}




\begin{tabular}{|c|c|c|c|}
\hline $\mathrm{H}$ & -4.86920500 & -4.45838700 & -2.80778500 \\
\hline $\mathrm{H}$ & -6.31088000 & -4.34556400 & -0.75213700 \\
\hline $\mathrm{H}$ & -2.44105900 & -3.76515100 & -2.57941000 \\
\hline $\mathrm{C}$ & -0.70795800 & 2.11987700 & -0.97949100 \\
\hline $\mathrm{O}$ & -0.30637700 & 1.05139900 & -1.42292700 \\
\hline $\mathrm{O}$ & 0.03554600 & 3.23230500 & -1.00066600 \\
\hline $\mathrm{C}$ & 1.38069100 & 3.11142400 & -1.48754000 \\
\hline $\mathrm{H}$ & 1.90479800 & 2.38142400 & -0.86644000 \\
\hline $\mathrm{H}$ & 1.35496900 & 2.74350700 & -2.51805100 \\
\hline $\mathrm{C}$ & 2.02550300 & 4.47215000 & -1.36912700 \\
\hline $\mathrm{H}$ & 2.00251200 & 4.80266900 & -0.32770800 \\
\hline $\mathrm{H}$ & 1.50346900 & 5.21045000 & -1.98171800 \\
\hline $\mathrm{H}$ & 3.06570600 & 4.40499600 & -1.69586800 \\
\hline $\mathrm{C}$ & -1.98052700 & 2.33688100 & -0.29505200 \\
\hline $\mathrm{N}$ & -2.31468300 & 3.64825300 & 0.08033700 \\
\hline $\mathrm{C}$ & -3.09945800 & 1.47584700 & -0.63579300 \\
\hline $\mathrm{O}$ & -4.29607700 & 2.02738500 & -0.33484100 \\
\hline $\mathrm{C}$ & -5.44706200 & 1.32645500 & -0.81150800 \\
\hline $\mathrm{H}$ & -5.31343300 & 1.09891200 & -1.87360900 \\
\hline $\mathrm{H}$ & -5.54950400 & 0.36802200 & -0.28662100 \\
\hline $\mathrm{C}$ & -6.64375500 & 2.21835400 & -0.56180100 \\
\hline $\mathrm{H}$ & -6.52546100 & 3.16729600 & -1.08824200 \\
\hline $\mathrm{H}$ & -6.74956100 & 2.42908500 & 0.50482400 \\
\hline $\mathrm{H}$ & -7.55617500 & 1.73174300 & -0.91442000 \\
\hline $\mathrm{O}$ & -3.01903200 & 0.33638900 & -1.07790900 \\
\hline $\mathrm{C}$ & -1.71329000 & 4.69029100 & 0.19524900 \\
\hline $\mathrm{O}$ & -1.27116100 & 5.76509100 & 0.35672300 \\
\hline $\mathrm{N}$ & -3.16510000 & -1.66881300 & 1.47442100 \\
\hline $\mathrm{H}$ & -2.38671200 & -0.89992300 & 1.20796700 \\
\hline $\mathrm{C}$ & -2.45950600 & -2.63493200 & 2.35075000 \\
\hline $\mathrm{H}$ & -1.97715300 & -2.06695700 & 3.14764100 \\
\hline $\mathrm{H}$ & -1.68955600 & -3.16077000 & 1.78264600 \\
\hline $\mathrm{H}$ & -3.16959900 & -3.34601200 & 2.77959700 \\
\hline $\mathrm{C}$ & -4.14255400 & -0.86941500 & 2.24305100 \\
\hline $\mathrm{H}$ & -3.60517400 & -0.35959700 & 3.04470400 \\
\hline $\mathrm{H}$ & -4.91981300 & -1.50343400 & 2.67315500 \\
\hline $\mathrm{H}$ & -4.58297900 & -0.12203000 & 1.57908100 \\
\hline $\mathrm{C}$ & 5.12577300 & -3.07861200 & 0.93334500 \\
\hline $\mathrm{C}$ & 4.56090400 & 1.24383900 & -1.51445400 \\
\hline $\mathrm{F}$ & 6.21783500 & -2.50359200 & 1.46099600 \\
\hline
\end{tabular}




$\begin{array}{lrrr}\text { F } & 5.55258800 & -4.02080200 & 0.08050400 \\ \text { F } & 4.47979200 & -3.70704100 & 1.92402700 \\ \text { F } & 5.84735000 & 1.12621600 & -1.86046700 \\ \text { F } & 4.49050800 & 2.22982800 & -0.59583500 \\ \text { F } & 3.88405500 & 1.68228600 & -2.58791900 \\ \text { C } & -1.44552800 & 1.28627000 & 1.51772300 \\ \text { H } & -2.37469100 & 1.68611700 & 1.95897600 \\ \text { O } & -1.34245200 & 0.05212600 & 1.24191900 \\ \text { C } & -0.21403000 & 2.04717300 & 1.85058600 \\ \text { C } & 2.12922000 & 3.47709300 & 2.32839800 \\ \mathrm{C} & -0.27613700 & 3.32046900 & 2.42007300 \\ \mathrm{C} & 1.02820400 & 1.47527200 & 1.55859200 \\ \mathrm{C} & 2.19627800 & 2.18883600 & 1.79502800 \\ \mathrm{C} & 0.89480100 & 4.03770300 & 2.64905200 \\ \mathrm{H} & -1.23962800 & 3.74867800 & 2.68297100 \\ \mathrm{H} & 1.05221600 & 0.47020700 & 1.14970500 \\ \mathrm{H} & 3.15775700 & 1.74830100 & 1.54864100 \\ \mathrm{H} & 0.84028500 & 5.03472600 & 3.07349800 \\ \mathrm{H} & 3.04163100 & 4.03840800 & 2.50411300\end{array}$

\section{TS-A (APFD)}

Zero-point correction= 0.689273 (Hartree/Particle)

Thermal correction to Energy= 0.737459

Thermal correction to Enthalpy=

0.738404

Thermal correction to Gibbs Free Energy=

0.605022

Sum of electronic and zero-point Energies= $-2907.339408$

Sum of electronic and thermal Energies= $-2907.291222$

Sum of electronic and thermal Enthalpies= $-2907.290278$

Sum of electronic and thermal Free Energies= $-2907.423659$

$\mathrm{E}(\mathrm{RAPFD})=-2908.02868119$

Imaginary frequency $=304 \mathrm{i}$

Relative energy $(A P F D / 6-311+G(d, p))=-2908.68602430$

01

$\begin{array}{llll}\text { C } & 3.88063800 & -2.30250500 & 0.22812100\end{array}$

$\begin{array}{llll}\mathrm{C} & 2.52751100 & -0.50142200 & -1.37102000\end{array}$

$\begin{array}{llll}\text { C } & 2.50569600 & -2.44375500 & 0.06390400\end{array}$ 


\begin{tabular}{|c|c|c|c|}
\hline $\mathrm{C}$ & 4.59270600 & -1.26986600 & -0.37009400 \\
\hline $\mathrm{C}$ & 3.89208700 & -0.36848600 & -1.16733000 \\
\hline $\mathrm{C}$ & 1.81358500 & -1.53343100 & -0.74206500 \\
\hline $\mathrm{H}$ & 1.96958300 & -3.24294900 & 0.55834500 \\
\hline $\mathrm{H}$ & 5.66088500 & -1.16797100 & -0.21782200 \\
\hline $\mathrm{H}$ & 1.99398100 & 0.20355300 & -1.99838100 \\
\hline $\mathrm{N}$ & 0.43333800 & -1.49153700 & -0.91256300 \\
\hline $\mathrm{H}$ & 0.09455600 & -0.55689600 & -1.15874200 \\
\hline $\mathrm{C}$ & -0.54371600 & -2.40921500 & -0.67153900 \\
\hline S & -0.29047800 & -4.03112800 & -0.26623900 \\
\hline $\mathrm{N}$ & -1.77383800 & -1.86617300 & -0.82909500 \\
\hline $\mathrm{H}$ & -1.85808000 & -0.86590000 & -1.02676100 \\
\hline $\mathrm{C}$ & -3.00971200 & -2.58165500 & -0.65228800 \\
\hline $\mathrm{C}$ & -5.18085500 & -2.71395600 & 0.59477400 \\
\hline $\mathrm{C}$ & -5.03314000 & -3.58134100 & -1.77597100 \\
\hline $\mathrm{C}$ & -5.93209400 & -2.93162900 & -0.72360400 \\
\hline $\mathrm{C}$ & -3.74946900 & -2.77657600 & -1.97946900 \\
\hline $\mathrm{C}$ & -3.93750200 & -1.86122000 & 0.33756200 \\
\hline $\mathrm{H}$ & -4.89094700 & -3.68535400 & 1.01688700 \\
\hline $\mathrm{H}$ & -4.77270700 & -4.59836100 & -1.45149200 \\
\hline $\mathrm{H}$ & -6.28725400 & -1.95992700 & -1.09602500 \\
\hline $\mathrm{H}$ & -3.97605100 & -1.79054200 & -2.40792000 \\
\hline $\mathrm{H}$ & -4.23820500 & -0.91809600 & -0.12897400 \\
\hline $\mathrm{H}$ & -2.74151600 & -3.57019700 & -0.26009500 \\
\hline $\mathrm{H}$ & -5.84514000 & -2.23107200 & 1.32067800 \\
\hline $\mathrm{H}$ & -5.57175400 & -3.68580500 & -2.72469100 \\
\hline $\mathrm{H}$ & -6.82436400 & -3.54223800 & -0.54476700 \\
\hline $\mathrm{H}$ & -3.07293500 & -3.28403800 & -2.67483100 \\
\hline $\mathrm{C}$ & -0.50114000 & 2.19450100 & -0.91703600 \\
\hline $\mathrm{O}$ & -0.25104500 & 1.14049400 & -1.49220400 \\
\hline $\mathrm{O}$ & 0.34699900 & 3.22602000 & -0.88172200 \\
\hline $\mathrm{C}$ & 1.65854900 & 3.02006600 & -1.43040000 \\
\hline $\mathrm{H}$ & 2.11288500 & 2.17952100 & -0.90469400 \\
\hline $\mathrm{H}$ & 1.56473700 & 2.76221800 & -2.48991500 \\
\hline $\mathrm{C}$ & 2.44408200 & 4.28863400 & -1.20647900 \\
\hline $\mathrm{H}$ & 2.48891800 & 4.52077000 & -0.13930500 \\
\hline $\mathrm{H}$ & 1.99048400 & 5.13620400 & -1.72757000 \\
\hline $\mathrm{H}$ & 3.46383400 & 4.15119300 & -1.57542600 \\
\hline $\mathrm{C}$ & -1.69874500 & 2.47249400 & -0.11530400 \\
\hline $\mathrm{N}$ & -1.88168100 & 3.77096600 & 0.36802700 \\
\hline
\end{tabular}




\begin{tabular}{|c|c|c|c|}
\hline $\mathrm{C}$ & -2.92434000 & 1.76583700 & -0.45912500 \\
\hline $\mathrm{O}$ & -4.02359300 & 2.41126300 & -0.00959200 \\
\hline $\mathrm{C}$ & -5.29364200 & 1.88487200 & -0.41458700 \\
\hline $\mathrm{H}$ & -5.32850100 & 0.80611900 & -0.22833300 \\
\hline $\mathrm{H}$ & -6.00686200 & 2.37496300 & 0.25279100 \\
\hline $\mathrm{C}$ & -5.59768000 & 2.18628200 & -1.87120400 \\
\hline $\mathrm{H}$ & -4.88983200 & 1.67092000 & -2.52421100 \\
\hline $\mathrm{H}$ & -5.53634200 & 3.26137600 & -2.06103700 \\
\hline $\mathrm{H}$ & -6.60878300 & 1.84917000 & -2.12078700 \\
\hline $\mathrm{O}$ & -2.98839800 & 0.67491900 & -1.01620200 \\
\hline $\mathrm{C}$ & -1.19638500 & 4.75773800 & 0.51448400 \\
\hline $\mathrm{O}$ & -0.67169200 & 5.79054800 & 0.71946000 \\
\hline $\mathrm{N}$ & -3.20158900 & -1.43458000 & 1.57043700 \\
\hline $\mathrm{H}$ & -2.31734600 & -0.71710100 & 1.28776700 \\
\hline $\mathrm{C}$ & -2.58158500 & -2.53897900 & 2.33119300 \\
\hline $\mathrm{H}$ & -1.97245800 & -2.10244200 & 3.12461400 \\
\hline $\mathrm{H}$ & -1.93014000 & -3.12224600 & 1.67698900 \\
\hline $\mathrm{H}$ & -3.34848200 & -3.18202900 & 2.77051200 \\
\hline $\mathrm{C}$ & -4.03066700 & -0.59162100 & 2.45158700 \\
\hline $\mathrm{H}$ & -3.39618500 & -0.19895200 & 3.24848800 \\
\hline $\mathrm{H}$ & -4.84749500 & -1.16300000 & 2.89737100 \\
\hline $\mathrm{H}$ & -4.43214000 & 0.24465500 & 1.87664000 \\
\hline $\mathrm{C}$ & 4.61936200 & -3.32633100 & 1.04894200 \\
\hline $\mathrm{C}$ & 4.61367500 & 0.81938500 & -1.73642200 \\
\hline $\mathrm{F}$ & 5.73282500 & -2.80751100 & 1.60480800 \\
\hline$F$ & 5.00657100 & -4.37325800 & 0.29531100 \\
\hline $\mathrm{F}$ & 3.85904300 & -3.81398500 & 2.04530900 \\
\hline $\mathrm{F}$ & 3.98483300 & 1.33990600 & -2.80823800 \\
\hline$F$ & 5.87117200 & 0.52190200 & -2.10289400 \\
\hline $\mathrm{F}$ & 4.70046100 & 1.81650000 & -0.82019000 \\
\hline $\mathrm{C}$ & -1.19796200 & 1.27262200 & 1.56436500 \\
\hline $\mathrm{H}$ & -2.05510600 & 1.71955500 & 2.09332600 \\
\hline $\mathrm{O}$ & -1.23561800 & 0.04394200 & 1.21274200 \\
\hline $\mathrm{C}$ & 0.11754800 & 1.85891400 & 1.90395300 \\
\hline $\mathrm{C}$ & 2.61821200 & 2.99851100 & 2.38273800 \\
\hline $\mathrm{C}$ & 0.21348700 & 3.08533800 & 2.56729500 \\
\hline $\mathrm{C}$ & 1.28275500 & 1.18310300 & 1.52354200 \\
\hline $\mathrm{C}$ & 2.52819300 & 1.75133700 & 1.75957200 \\
\hline $\mathrm{C}$ & 1.46175700 & 3.65830900 & 2.79656200 \\
\hline $\mathrm{H}$ & -0.68898200 & 3.58982500 & 2.90161800 \\
\hline
\end{tabular}




$\begin{array}{llll}\mathrm{H} & 1.18674300 & 0.21551300 & 1.04640300 \\ \mathrm{H} & 3.42835100 & 1.23166600 & 1.44499400 \\ \mathrm{H} & 1.53004800 & 4.62133400 & 3.29458700 \\ \mathrm{H} & 3.59200700 & 3.44864400 & 2.55719500\end{array}$

\section{TS-A ( $\omega$ B97XD)}

Zero-point correction= 0.695504 (Hartree/Particle)

Thermal correction to Energy=

0.743393

Thermal correction to Enthalpy=

0.744337

Thermal correction to Gibbs Free Energy=

0.611740

Sum of electronic and zero-point Energies=

$-2908.571937$

Sum of electronic and thermal Energies=

$-2908.524047$

Sum of electronic and thermal Enthalpies=

$-2908.523103$

Sum of electronic and thermal Free Energies=

$-2908.655701$

$\mathrm{E}(\omega \mathrm{B} 97 \mathrm{XD})=-2909.26744052$

Imaginary frequency $=245 \mathrm{i}$

Relative energy $(\omega \mathrm{B} 97 \mathrm{XD} / 6-311+\mathrm{G}(\mathrm{d}, \mathrm{p}))=-2909.93135513$

01

$\mathrm{C}$

C

$\mathrm{C}$

$\mathrm{C}$

$\mathrm{C}$

$\mathrm{C}$

$\mathrm{H}$

H

$\mathrm{H}$

$\mathrm{N}$

$\mathrm{H}$

$\mathrm{C}$

S

$\mathrm{N}$

H

$\mathrm{C}$

C

$\begin{array}{rrr}4.02512700 & -2.15635000 & 0.31032500 \\ 2.60644300 & -0.44163800 & -1.32399500 \\ 2.65665800 & -2.35347900 & 0.14475800 \\ 4.69400600 & -1.11016800 & -0.31016900 \\ 3.96390600 & -0.25121700 & -1.12617000 \\ 1.93502100 & -1.48883600 & -0.67919000 \\ 2.15158000 & -3.16326500 & 0.65193100 \\ 5.75619300 & -0.96382700 & -0.15955200 \\ 2.04107600 & 0.23172100 & -1.95769900 \\ 0.54676200 & -1.50303000 & -0.85560900 \\ 0.17374500 & -0.58527200 & -1.10876700 \\ -0.39034200 & -2.46484900 & -0.64501900 \\ -0.07294100 & -4.07151500 & -0.22766500 \\ -1.64157600 & -1.98574100 & -0.83771000 \\ -1.76590200 & -0.99633400 & -1.06202600 \\ -2.85387500 & -2.75720200 & -0.70407500 \\ -5.08991400 & -2.93153800 & 0.43800700 \\ -4.79162500 & -3.84502400 & -1.90534300\end{array}$

$\mathrm{C}$

$$
-4.79162500 \quad-3.84502400
$$

$-1.90534300$ 


\begin{tabular}{|c|c|c|c|}
\hline $\mathrm{C}$ & -5.76390200 & -3.20525000 & -0.91240100 \\
\hline $\mathrm{C}$ & -3.52147800 & -3.00759500 & -2.06156700 \\
\hline $\mathrm{C}$ & -3.85798200 & -2.05259800 & 0.22538300 \\
\hline $\mathrm{H}$ & -4.79972100 & -3.88309200 & 0.90078900 \\
\hline $\mathrm{H}$ & -4.52007100 & -4.84724600 & -1.54953900 \\
\hline $\mathrm{H}$ & -6.13412200 & -2.25664400 & -1.32352900 \\
\hline $\mathrm{H}$ & -3.75212000 & -2.04024200 & -2.52573600 \\
\hline $\mathrm{H}$ & -4.15506100 & -1.12206100 & -0.26641100 \\
\hline $\mathrm{H}$ & -2.56895300 & -3.72526200 & -0.28087000 \\
\hline $\mathrm{H}$ & -5.80595400 & -2.44642400 & 1.10889300 \\
\hline $\mathrm{H}$ & -5.27764500 & -3.97715100 & -2.87661200 \\
\hline $\mathrm{H}$ & -6.63735500 & -3.84654800 & -0.76166700 \\
\hline $\mathrm{H}$ & -2.79763800 & -3.51494000 & -2.70504600 \\
\hline $\mathrm{C}$ & -0.56173500 & 2.16987900 & -0.98617600 \\
\hline $\mathrm{O}$ & -0.22635900 & 1.12411000 & -1.52716100 \\
\hline $\mathrm{O}$ & 0.21828100 & 3.25204500 & -0.97218400 \\
\hline $\mathrm{C}$ & 1.55204800 & 3.11170300 & -1.48574200 \\
\hline $\mathrm{H}$ & 2.03261100 & 2.28058300 & -0.96722100 \\
\hline $\mathrm{H}$ & 1.49987200 & 2.87648300 & -2.55252400 \\
\hline $\mathrm{C}$ & 2.27939400 & 4.40736800 & -1.21459900 \\
\hline $\mathrm{H}$ & 2.30023400 & 4.60659700 & -0.14049900 \\
\hline $\mathrm{H}$ & 1.79525000 & 5.24766600 & -1.71781300 \\
\hline $\mathrm{H}$ & 3.30735100 & 4.32082000 & -1.57426200 \\
\hline $\mathrm{C}$ & -1.79210700 & 2.37997400 & -0.21301100 \\
\hline $\mathrm{N}$ & -2.07150500 & 3.68884500 & 0.22412600 \\
\hline $\mathrm{C}$ & -2.97140400 & 1.61584000 & -0.60750900 \\
\hline $\mathrm{O}$ & -4.12171600 & 2.19948100 & -0.21635100 \\
\hline $\mathrm{C}$ & -5.33136500 & 1.60211400 & -0.69057900 \\
\hline $\mathrm{H}$ & -5.23852900 & 1.40307200 & -1.76194700 \\
\hline $\mathrm{H}$ & -5.49198000 & 0.63703800 & -0.19418800 \\
\hline $\mathrm{C}$ & -6.45932500 & 2.56411900 & -0.38562600 \\
\hline $\mathrm{H}$ & -6.28966100 & 3.52252000 & -0.88099600 \\
\hline $\mathrm{H}$ & -6.54038200 & 2.74271400 & 0.68942300 \\
\hline $\mathrm{H}$ & -7.40649600 & 2.14991200 & -0.74171300 \\
\hline $\mathrm{O}$ & -2.96979600 & 0.52749600 & -1.16886900 \\
\hline C & -1.46258600 & 4.72387500 & 0.34862800 \\
\hline $\mathrm{O}$ & -1.01523000 & 5.79556400 & 0.53015500 \\
\hline $\mathrm{N}$ & -3.20344400 & -1.59189400 & 1.49704800 \\
\hline $\mathrm{H}$ & -2.38912300 & -0.87967600 & 1.24863300 \\
\hline $\mathrm{C}$ & -2.57109900 & -2.66167300 & 2.30459400 \\
\hline
\end{tabular}




$\begin{array}{lrrr}\mathrm{H} & -2.04328300 & -2.18711000 & 3.13246000 \\ \mathrm{H} & -1.84694500 & -3.20785900 & 1.69784200 \\ \mathrm{H} & -3.33183600 & -3.34266000 & 2.69190000 \\ \mathrm{C} & -4.09442300 & -0.75309600 & 2.32886100 \\ \mathrm{H} & -3.49380500 & -0.30965800 & 3.12424300 \\ \mathrm{H} & -4.89927700 & -1.34388500 & 2.76801800 \\ \mathrm{H} & -4.50481700 & 0.04704800 & 1.71089900 \\ \mathrm{C} & 4.80130600 & -3.13125100 & 1.15716900 \\ \mathrm{C} & 4.64331200 & 0.94566000 & -1.73073600 \\ \mathrm{~F} & 5.90732700 & -2.56819800 & 1.67519000 \\ \mathrm{~F} & 5.20007400 & -4.19493100 & 0.44008600 \\ \mathrm{~F} & 4.06943900 & -3.59632500 & 2.18135700 \\ \mathrm{~F} & 5.90591400 & 0.67917100 & -2.09405000 \\ \mathrm{~F} & 4.69998800 & 1.96709300 & -0.84711500 \\ \mathrm{~F} & 3.99646200 & 1.40987300 & -2.81290300 \\ \mathrm{C} & -1.28837300 & 1.28519000 & 1.51889400 \\ \mathrm{H} & -2.16081100 & 1.74256400 & 2.01599200 \\ \mathrm{O} & -1.29048300 & 0.04347200 & 1.24374200 \\ \mathrm{C} & 0.01656400 & 1.92053500 & 1.86423100 \\ \mathrm{C} & 2.48992000 & 3.10046500 & 2.38298600 \\ \mathrm{C} & 0.08166200 & 3.16232300 & 2.49655300 \\ \mathrm{C} & 1.19856000 & 1.25302600 & 1.53397200 \\ \mathrm{C} & 2.43015100 & 1.84130200 & 1.78585400 \\ \mathrm{C} & 1.31601100 & 3.75394200 & 2.74805100 \\ \mathrm{H} & -0.83285000 & 3.66664400 & 2.79362400 \\ \mathrm{H} & 1.12616200 & 0.27226400 & 1.08113700 \\ \mathrm{H} & 3.34263300 & 1.32433900 & 1.50677200 \\ \mathrm{H} & 1.35945300 & 4.72695800 & 3.22596000 \\ & 3.45184100 & 3.56637500 & 2.57187100\end{array}$

\section{TS-A-int1 ( $\omega$ B97XD)}

substrate

$\begin{array}{lc}\text { Zero-point correction= } & 0.697996 \text { (Hartree/Particle) } \\ \text { Thermal correction to Energy= } & 0.746808 \\ \text { Thermal correction to Enthalpy= } & 0.747752 \\ \text { Thermal correction to Gibbs Free Energy= } & 0.613225 \\ \text { Sum of electronic and zero-point Energies= } & -2908.580706 \\ \text { Sum of electronic and thermal Energies= } & -2908.531894 \\ \text { Sum of electronic and thermal Enthalpies= } & -2908.530950 \\ \text { Sum of electronic and thermal Free Energies }= & -2908.665477\end{array}$


$\mathrm{E}(\omega \mathrm{B} 97 \mathrm{XD})=-2909.27870234$

Relative energy $(\omega \mathrm{B} 97 \mathrm{XD} / 6-311+\mathrm{G}(\mathrm{d}, \mathrm{p}))=-2909.94300201$

01

$\mathrm{C}$

$\mathrm{C}$

$\mathrm{C}$

$\mathrm{C}$

$\mathrm{C}$

$\mathrm{C}$

$\mathrm{H}$

$\mathrm{H}$

$\mathrm{H}$

$\mathrm{N}$

$\mathrm{H}$

$\mathrm{C}$

S

$\mathrm{N}$

$\mathrm{H}$

C

C

C

C

C

C

$\mathrm{H}$

$\mathrm{H}$

$\mathrm{H}$

$\mathrm{H}$

$\mathrm{H}$

$\mathrm{H}$

$\mathrm{H}$

$\mathrm{H}$

$\mathrm{H}$

$\mathrm{H}$

C

O

O

C

$\begin{array}{rrr}4.01748100 & -2.03426300 & -0.17675600 \\ 2.45945900 & -0.16372700 & -1.46871300 \\ 2.63855400 & -2.22031900 & -0.22441500 \\ 4.63126900 & -0.92560900 & -0.74312300 \\ 3.83011800 & 0.00946700 & -1.38932600 \\ 1.84278100 & -1.26772800 & -0.86505300 \\ 2.18273700 & -3.08826500 & 0.22974200 \\ 5.70509400 & -0.79522700 & -0.68828000 \\ 1.84120000 & 0.56206500 & -1.98383400 \\ 0.44656800 & -1.26890800 & -0.94505800 \\ 0.07531600 & -0.37601700 & -1.29662200 \\ -0.49965900 & -2.15813600 & -0.55184800 \\ -0.21455000 & -3.67118700 & 0.14326000 \\ -1.75497400 & -1.67836700 & -0.77402300 \\ -1.88056400 & -0.77978300 & -1.25415700 \\ -2.96164700 & -2.46811000 & -0.64800900 \\ -5.22505900 & -2.58887900 & 0.47955600 \\ -4.92364400 & -3.50860100 & -1.85495600 \\ -5.88930500 & -2.82802800 & -0.88206900 \\ -3.62813300 & -2.70887200 & -2.00816500 \\ -3.96300700 & -1.75964200 & 0.27528300 \\ -4.97721200 & -3.55589500 & 0.93414200 \\ -4.69051300 & -4.51596600 & -1.48583500 \\ -6.20800400 & -1.86428200 & -1.29951300 \\ -3.83080100 & -1.73696400 & -2.47245000 \\ -4.21628300 & -0.81772300 & -0.21424300 \\ -2.66991100 & -3.43173900 & -0.22124000 \\ -5.92329300 & -2.07749600 & 1.15118300 \\ -5.40131800 & -3.63389500 & -2.83109300 \\ -6.79161900 & -3.43144000 & -0.74728400 \\ -2.91735100 & -3.24105400 & -2.64642500 \\ -0.88495700 & 2.12771800 & -0.94112200 \\ 1.29813800 & 3.09213200 & -1.04145500\end{array}$




\begin{tabular}{|c|c|c|c|}
\hline $\mathrm{H}$ & 1.86640400 & 2.33795200 & -0.48738500 \\
\hline $\mathrm{H}$ & 1.29391300 & 2.81548200 & -2.09855800 \\
\hline $\mathrm{C}$ & 1.88843400 & 4.46910100 & -0.83197600 \\
\hline $\mathrm{H}$ & 1.82453400 & 4.75989900 & 0.21934000 \\
\hline $\mathrm{H}$ & 1.35644100 & 5.21573200 & -1.42635600 \\
\hline $\mathrm{H}$ & 2.93960600 & 4.45956100 & -1.12991500 \\
\hline $\mathrm{C}$ & -2.18802300 & 2.30561500 & -0.39230600 \\
\hline $\mathrm{N}$ & -2.42509400 & 3.35715900 & 0.51185500 \\
\hline $\mathrm{C}$ & -3.29617200 & 1.54013200 & -0.82960900 \\
\hline $\mathrm{O}$ & -4.47889800 & 1.95987500 & -0.27274500 \\
\hline $\mathrm{C}$ & -5.67777200 & 1.43816000 & -0.84044400 \\
\hline $\mathrm{H}$ & -5.53299100 & 1.26217500 & -1.90903400 \\
\hline $\mathrm{H}$ & -5.91513900 & 0.46844300 & -0.37917200 \\
\hline $\mathrm{C}$ & -6.78558000 & 2.43606500 & -0.57085700 \\
\hline $\mathrm{H}$ & -6.55717100 & 3.39505800 & -1.04108100 \\
\hline $\mathrm{H}$ & -6.90943100 & 2.60212800 & 0.50238600 \\
\hline $\mathrm{H}$ & -7.73106000 & 2.06418200 & -0.97618300 \\
\hline $\mathrm{O}$ & -3.28851300 & 0.57555100 & -1.60549000 \\
\hline $\mathrm{C}$ & -1.79216200 & 4.23858000 & 1.03044200 \\
\hline $\mathrm{O}$ & -1.28259200 & 5.11932500 & 1.63409300 \\
\hline $\mathrm{N}$ & -3.29414200 & -1.34330900 & 1.56021400 \\
\hline $\mathrm{H}$ & -2.38112300 & -0.91495500 & 1.30745200 \\
\hline $\mathrm{C}$ & -2.94212800 & -2.45950000 & 2.47822700 \\
\hline $\mathrm{H}$ & -2.33533600 & -2.04595600 & 3.28286700 \\
\hline $\mathrm{H}$ & -2.34543300 & -3.19401400 & 1.93511600 \\
\hline $\mathrm{H}$ & -3.85328300 & -2.90238100 & 2.88046300 \\
\hline $\mathrm{C}$ & -4.03003600 & -0.26287200 & 2.26876600 \\
\hline $\mathrm{H}$ & -3.40944300 & 0.07548500 & 3.09824900 \\
\hline $\mathrm{H}$ & -4.97575400 & -0.65115200 & 2.64794200 \\
\hline $\mathrm{H}$ & -4.20282000 & 0.56688400 & 1.58016900 \\
\hline $\mathrm{C}$ & 4.85914500 & -3.00914100 & 0.60147500 \\
\hline $\mathrm{C}$ & 4.44821900 & 1.26825600 & -1.93150700 \\
\hline $\mathrm{F}$ & 5.03804300 & -2.58233100 & 1.87280000 \\
\hline $\mathrm{F}$ & 6.08362600 & -3.14952200 & 0.06858200 \\
\hline $\mathrm{F}$ & 4.30281600 & -4.22392000 & 0.67322800 \\
\hline $\mathrm{F}$ & 5.68553500 & 1.05816100 & -2.40491300 \\
\hline $\mathrm{F}$ & 4.55473100 & 2.20802200 & -0.96663600 \\
\hline $\mathrm{F}$ & 3.71938800 & 1.81062500 & -2.91974300 \\
\hline $\mathrm{C}$ & -0.42557400 & 0.93043400 & 2.06215600 \\
\hline $\mathrm{H}$ & -1.12052300 & 1.78803600 & 2.06522100 \\
\hline
\end{tabular}




$\begin{array}{lrrr}\mathrm{O} & -0.85699900 & -0.21321700 & 1.98221400 \\ \mathrm{C} & 0.99786600 & 1.25171700 & 2.15980900 \\ \mathrm{C} & 3.69466300 & 1.87757200 & 2.26852100 \\ \mathrm{C} & 1.39423600 & 2.58882600 & 2.26552800 \\ \mathrm{C} & 1.95173300 & 0.22756300 & 2.12588500 \\ \mathrm{C} & 3.30050500 & 0.54156300 & 2.18027800 \\ \mathrm{C} & 2.74767200 & 2.89798800 & 2.32081300 \\ \mathrm{H} & 0.64851400 & 3.37942100 & 2.28074100 \\ \mathrm{H} & 1.61467100 & -0.80073900 & 2.04649300 \\ \mathrm{H} & 4.04137400 & -0.24841400 & 2.14542500 \\ \mathrm{H} & 3.06503900 & 3.93261200 & 2.39353400 \\ \mathrm{H} & 4.75134200 & 2.12326200 & 2.29657800\end{array}$

TS-A-int2 ( $\omega B$ 97XD)

Zero-point correction=

0.696469 (Hartree/Particle)

Thermal correction to Energy=

0.744897

Thermal correction to Enthalpy=

0.745841

Thermal correction to Gibbs Free Energy=

0.610090

Sum of electronic and zero-point Energies=

$-2908.592116$

Sum of electronic and thermal Energies=

$-2908.543688$

Sum of electronic and thermal Enthalpies=

$-2908.542744$

Sum of electronic and thermal Free Energies=

$-2908.678495$

$\mathrm{E}(\omega \mathrm{B} 97 \mathrm{XD})=-2909.28858505$

Relative energy $(\omega \mathrm{B} 97 \mathrm{XD} / 6-311+\mathrm{G}(\mathrm{d}, \mathrm{p}))=-2909.94830894$

$\begin{array}{rrrr}01 & & & \\ \mathrm{C} & 3.88051000 & -2.26368200 & 0.31937100 \\ \mathrm{C} & 2.53092100 & -0.58756200 & -1.40916100 \\ \mathrm{C} & 2.51592400 & -2.45079300 & 0.12018300 \\ \mathrm{C} & 4.58087700 & -1.24524200 & -0.31300100 \\ \mathrm{C} & 3.88493400 & -0.40537200 & -1.17642200 \\ \mathrm{C} & 1.82579200 & -1.60492600 & -0.75083900 \\ \mathrm{H} & 1.98665400 & -3.23897300 & 0.63673800 \\ \mathrm{H} & 5.64035400 & -1.10716300 & -0.13708300 \\ \mathrm{H} & 1.99850700 & 0.07196800 & -2.08438500 \\ \mathrm{~N} & 0.44413000 & -1.61752000 & -0.96574700 \\ \mathrm{H} & 0.08499500 & -0.70149700 & -1.22507100 \\ \mathrm{C} & -0.51474700 & -2.55057000 & -0.67985300\end{array}$


H

H

$\mathrm{H}$

$\mathrm{H}$

H

$\mathrm{H}$

$\mathrm{H}$

$\mathrm{H}$

H

C

O

O

C

$\mathrm{H}$

$\mathrm{H}$

C

$\mathrm{H}$

$\mathrm{H}$

$\mathrm{H}$

C

$\mathrm{N}$

C

O

C

H

$\mathrm{H}$

C

$\mathrm{H}$

$\mathrm{H}$

$\mathrm{H}$ $\begin{array}{lll}-0.21729900 & -4.15063300 & -0.24168300\end{array}$

$\begin{array}{lll}-1.75692500 & -2.04424800 & -0.82990200\end{array}$

$\begin{array}{lll}-1.85530100 & -1.06424500 & -1.08243500\end{array}$

$\begin{array}{lll}-2.99223900 & -2.75576800 & -0.58183500\end{array}$

$\begin{array}{lll}-5.20953500 & -2.73995900 & 0.59111600\end{array}$

$\begin{array}{lll}-4.98756000 & -3.89494700 & -1.64384600\end{array}$

$\begin{array}{lll}-5.91761700 & -3.11433400 & -0.71457500\end{array}$

$\begin{array}{lll}-3.69036300 & -3.12598900 & -1.89667100\end{array}$

$\begin{array}{lll}-3.93824100 & -1.92406500 & 0.30711600\end{array}$

$\begin{array}{lll}-4.94934100 & -3.65396500 & 1.13991600\end{array}$

$-4.74866600 \quad-4.86303800 \quad-1.18518700$

$\begin{array}{lll}-6.24695500 & -2.19542800 & -1.21920700\end{array}$

$\begin{array}{lll}-3.89997100 & -2.20448500 & -2.45655400\end{array}$

$\begin{array}{lll}-4.23013200 & -1.04344400 & -0.27982900\end{array}$

$\begin{array}{lll}-2.72204300 & -3.68395500 & -0.06999300\end{array}$

$\begin{array}{lll}-5.89186100 & -2.16737100 & 1.22763800\end{array}$

$\begin{array}{lll}-5.48790900 & -4.11033900 & -2.59320200\end{array}$

$\begin{array}{lll}-6.82053500 & -3.69384900 & -0.49703600\end{array}$

$\begin{array}{lll}-2.99571100 & -3.72104300 & -2.49648400\end{array}$

$\begin{array}{lll}-0.40089400 & 2.17686900 & -1.02712100\end{array}$

$\begin{array}{lll}-0.12703600 & 1.17021200 & -1.64183000\end{array}$

$\begin{array}{lll}0.34503300 & 3.26687900 & -1.02075800\end{array}$

$\begin{array}{lll}1.67402000 & 3.15353700 & -1.57860500\end{array}$

$\begin{array}{lll}2.12942600 & 2.25434600 & -1.16219800\end{array}$

$\begin{array}{lll}1.58810400 & 3.03809300 & -2.66167000\end{array}$

$2.43975900 \quad 4.38860400 \quad-1.17238500$

$2.48442300 \quad 4.46025200 \quad-0.08273200$

$\begin{array}{lll}1.97524800 & 5.29455000 & -1.56808300\end{array}$

$3.45907700 \quad 4.31359200 \quad-1.55781900$

$\begin{array}{lll}-1.58970100 & 2.30740400 & -0.06237000\end{array}$

$\begin{array}{lll}-1.87889900 & 3.65548500 & 0.30636900\end{array}$

$\begin{array}{lll}-2.84939900 & 1.66605500 & -0.65723500\end{array}$

$\begin{array}{lll}-3.94948200 & 2.33178200 & -0.32693300\end{array}$

$\begin{array}{lll}-5.18590700 & 1.82833100 & -0.87008600\end{array}$

$\begin{array}{lll}-5.06636100 & 1.69872000 & -1.94866400\end{array}$

$\begin{array}{lll}-5.38651800 & 0.84364200 & -0.43636500\end{array}$

$\begin{array}{lll}-6.26797100 & 2.82538500 & -0.52427900\end{array}$

$-6.04928200 \quad 3.80207100 \quad-0.96136500$

$\begin{array}{lll}-6.36120800 & 2.94214900 & 0.55796100\end{array}$

$\begin{array}{lll}-7.22535000 & 2.47526900 & -0.91883900\end{array}$ 


\begin{tabular}{|c|c|c|c|}
\hline $\mathrm{O}$ & -2.85807700 & 0.65118700 & -1.31823400 \\
\hline $\mathrm{C}$ & -1.24343400 & 4.68229200 & 0.38641600 \\
\hline $\mathrm{O}$ & -0.77813200 & 5.74822200 & 0.51546500 \\
\hline $\mathrm{N}$ & -3.25544200 & -1.37909600 & 1.49723400 \\
\hline $\mathrm{H}$ & -1.91902400 & -0.37784900 & 1.10590600 \\
\hline $\mathrm{C}$ & -2.65254500 & -2.38040100 & 2.37947500 \\
\hline $\mathrm{H}$ & -2.07866400 & -1.86044400 & 3.15108600 \\
\hline $\mathrm{H}$ & -1.95755300 & -3.01255700 & 1.82367700 \\
\hline $\mathrm{H}$ & -3.40128800 & -3.01579700 & 2.87483100 \\
\hline $\mathrm{C}$ & -4.09466000 & -0.45904400 & 2.25833500 \\
\hline $\mathrm{H}$ & -3.48163000 & 0.03493800 & 3.01837900 \\
\hline $\mathrm{H}$ & -4.93239000 & -0.94965900 & 2.77525800 \\
\hline $\mathrm{H}$ & -4.49822000 & 0.31143600 & 1.59415000 \\
\hline $\mathrm{C}$ & 4.62258200 & -3.22032700 & 1.21678700 \\
\hline $\mathrm{C}$ & 4.59880700 & 0.75982700 & -1.80141500 \\
\hline $\mathrm{F}$ & 5.70374900 & -2.64165000 & 1.77036600 \\
\hline $\mathrm{F}$ & 5.05693000 & -4.29325800 & 0.53528100 \\
\hline $\mathrm{F}$ & 3.85080000 & -3.67032400 & 2.21674000 \\
\hline $\mathrm{F}$ & 5.86024300 & 0.46098300 & -2.13824600 \\
\hline $\mathrm{F}$ & 4.66380000 & 1.80479900 & -0.94255600 \\
\hline $\mathrm{F}$ & 3.97742400 & 1.21295300 & -2.90391700 \\
\hline $\mathrm{C}$ & -1.18554500 & 1.47088200 & 1.26806200 \\
\hline $\mathrm{H}$ & -2.00137400 & 1.70227300 & 1.96822300 \\
\hline $\mathrm{O}$ & -1.06559000 & 0.12794100 & 0.99406500 \\
\hline $\mathrm{C}$ & 0.12773700 & 2.00146600 & 1.81038600 \\
\hline $\mathrm{C}$ & 2.58796200 & 3.04466100 & 2.62890900 \\
\hline $\mathrm{C}$ & 0.17699100 & 3.13016000 & 2.62673900 \\
\hline $\mathrm{C}$ & 1.31939500 & 1.37444300 & 1.44034200 \\
\hline $\mathrm{C}$ & 2.54359800 & 1.89738500 & 1.83919200 \\
\hline $\mathrm{C}$ & 1.40264400 & 3.65239500 & 3.03179700 \\
\hline $\mathrm{H}$ & -0.74476700 & 3.60532500 & 2.95016100 \\
\hline $\mathrm{H}$ & 1.27709800 & 0.47197100 & 0.84313500 \\
\hline $\mathrm{H}$ & 3.46075500 & 1.40987200 & 1.52533900 \\
\hline $\mathrm{H}$ & 1.42849100 & 4.53514200 & 3.66229700 \\
\hline $\mathrm{H}$ & 3.54285600 & 3.45567500 & 2.94037300 \\
\hline
\end{tabular}

\section{TS-B (B3LYP)}

Zero-point correction=

0.686705 (Hartree/Particle)

Thermal correction to Energy=

0.735883

Thermal correction to Enthalpy=

0.736827 
Thermal correction to Gibbs Free Energy=

Sum of electronic and zero-point Energies=

Sum of electronic and thermal Energies=

Sum of electronic and thermal Enthalpies=

Sum of electronic and thermal Free Energies=

$\mathrm{E}(\mathrm{RB} 3 \mathrm{LYP})=-2909.91560984$

Imaginary frequency $=247 \mathrm{i}$
0.597339

$-2909.228904$

$-2909.179727$

$-2909.178783$

$-2909.318271$

Relative energy $(\mathrm{B} 3 \mathrm{LYP} / 6-311+\mathrm{G}(\mathrm{d}, \mathrm{p}))=-2910.67892377$

01

C

C

C

C

C

C

$\mathrm{H}$

H

$\mathrm{H}$

N

$\mathrm{H}$

C

S

$\mathrm{N}$

$\mathrm{H}$

C

C

C

C

C

C

$\mathrm{H}$

$\mathrm{H}$

$\mathrm{H}$

$\mathrm{H}$

$\mathrm{H}$

$\mathrm{H}$

$\mathrm{H}$

$\mathrm{H}$

$\begin{array}{rrc}-4.78116300 & 1.37555300 & 0.42815300 \\ -3.00092700 & -0.02900000 & -1.16010300 \\ -3.44051700 & 1.76498600 & 0.39905500 \\ -5.24738800 & 0.28866800 & -0.30972500 \\ -4.33696300 & -0.41224400 & -1.09998000 \\ -2.53290600 & 1.06142300 & -0.40649600 \\ -3.10354800 & 2.60163400 & 0.99244600 \\ -6.28851500 & -0.00603000 & -0.26662100 \\ -2.30514400 & -0.58368800 & -1.77864400 \\ -1.15091800 & 1.31597200 & -0.50763400 \\ -0.64569900 & 0.53924400 & -0.94355800 \\ -0.38352100 & 2.39350300 & -0.15431000 \\ -0.95788400 & 3.83418700 & 0.52078900 \\ 0.94984900 & 2.17670300 & -0.39622100 \\ 1.22618100 & 1.33717900 & -0.91502000 \\ 1.93115700 & 3.25516100 & -0.41572000 \\ 4.27344300 & 4.01290400 & 0.11900700 \\ 3.04813100 & 5.03574500 & -1.85615300 \\ 4.41011400 & 4.63153300 & -1.28324800 \\ 2.07456400 & 3.85199700 & -1.83270800 \\ 3.31505100 & 2.80753900 & 0.10407200 \\ 3.89983500 & 4.77423200 & 0.81781800 \\ 2.63094600 & 5.86189600 & -1.26344300 \\ 4.88617700 & 3.89996100 & -1.95183200 \\ 2.41488500 & 3.06489100 & -2.51967500 \\ 3.70785100 & 2.03914100 & -0.57102000 \\ 1.53664000 & 4.03914700 & 0.23648700 \\ 5.26476500 & 3.71216100 & 0.47216100 \\ 3.16123400 & 5.41100900 & -2.88010600\end{array}$




\begin{tabular}{|c|c|c|c|}
\hline $\mathrm{H}$ & 5.08440200 & 5.49452400 & -1.23298500 \\
\hline $\mathrm{H}$ & 1.07872500 & 4.16458200 & -2.16376000 \\
\hline $\mathrm{C}$ & 0.60404400 & -2.13618900 & -1.11543500 \\
\hline $\mathrm{O}$ & -0.07794400 & -1.18148800 & -1.49073400 \\
\hline $\mathrm{O}$ & 0.14274000 & -3.39165600 & -1.10813500 \\
\hline $\mathrm{C}$ & -1.23954900 & -3.58247200 & -1.49595700 \\
\hline $\mathrm{H}$ & -1.87790400 & -3.00855700 & -0.81920000 \\
\hline $\mathrm{H}$ & -1.38430300 & -3.19043200 & -2.50747500 \\
\hline $\mathrm{C}$ & -1.53504700 & -5.06694500 & -1.41205600 \\
\hline $\mathrm{H}$ & -1.36130900 & -5.43916900 & -0.39777100 \\
\hline $\mathrm{H}$ & -0.90353700 & -5.63486500 & -2.10235200 \\
\hline $\mathrm{H}$ & -2.58442600 & -5.24068600 & -1.67298500 \\
\hline $\mathrm{C}$ & 1.96598300 & -2.00645500 & -0.54501600 \\
\hline $\mathrm{N}$ & 2.57458200 & -3.23858100 & -0.22906700 \\
\hline $\mathrm{C}$ & 2.83756600 & -1.02539500 & -1.25132600 \\
\hline $\mathrm{O}$ & 4.09576800 & -1.47811700 & -1.39377400 \\
\hline $\mathrm{C}$ & 5.05237700 & -0.60496900 & -2.02767400 \\
\hline $\mathrm{H}$ & 4.62305000 & -0.22288700 & -2.95855100 \\
\hline $\mathrm{H}$ & 5.23847400 & 0.25127600 & -1.36853900 \\
\hline $\mathrm{C}$ & 6.31397800 & -1.41500900 & -2.26200100 \\
\hline $\mathrm{H}$ & 6.11492700 & -2.25683100 & -2.93213700 \\
\hline $\mathrm{H}$ & 6.70237600 & -1.81286200 & -1.31948000 \\
\hline $\mathrm{H}$ & 7.08322800 & -0.78299500 & -2.71921800 \\
\hline $\mathrm{O}$ & 2.50459600 & 0.09515800 & -1.62444500 \\
\hline $\mathrm{C}$ & 3.62975800 & -3.63588800 & 0.20118800 \\
\hline $\mathrm{O}$ & 4.60951500 & -4.16727800 & 0.59475300 \\
\hline $\mathrm{N}$ & 3.23772700 & 2.09314000 & 1.44599600 \\
\hline $\mathrm{H}$ & 2.84274100 & 1.04881600 & 1.29680000 \\
\hline $\mathrm{C}$ & 2.38564200 & 2.74773800 & 2.47932400 \\
\hline $\mathrm{H}$ & 2.35761300 & 2.08693000 & 3.34812800 \\
\hline $\mathrm{H}$ & 1.37299800 & 2.88528300 & 2.10114700 \\
\hline $\mathrm{H}$ & 2.81044700 & 3.71355200 & 2.76556000 \\
\hline $\mathrm{C}$ & 4.58348500 & 1.77956500 & 2.01261300 \\
\hline $\mathrm{H}$ & 4.44158500 & 1.02412000 & 2.78573200 \\
\hline $\mathrm{H}$ & 5.04996200 & 2.67349200 & 2.43053900 \\
\hline $\mathrm{H}$ & 5.21165700 & 1.35800000 & 1.22589600 \\
\hline $\mathrm{C}$ & -5.75627200 & 2.18706000 & 1.24288600 \\
\hline $\mathrm{C}$ & -4.76550100 & -1.64407300 & -1.84996100 \\
\hline $\mathrm{F}$ & -6.26977200 & 3.21221700 & 0.52523700 \\
\hline $\mathrm{F}$ & -5.17780400 & 2.72299200 & 2.33832100 \\
\hline
\end{tabular}




$\begin{array}{lrrc}\text { F } & -6.80257700 & 1.43860700 & 1.66135600 \\ \text { F } & -6.09376600 & -1.67091900 & -2.07685100 \\ \text { F } & -4.46143700 & -2.77627100 & -1.15783000 \\ \text { F } & -4.14188100 & -1.75290300 & -3.04473900 \\ \text { C } & 1.63741900 & -0.98986500 & 1.16205000 \\ \text { O } & 2.73668700 & -0.38494200 & 1.49059100 \\ \text { H } & 0.83322800 & -0.38062400 & 0.72483700 \\ \text { C } & 1.12128000 & -2.05018500 & 2.08508300 \\ \text { C } & 0.14985400 & -3.99490500 & 3.85636400 \\ \text { C } & -0.23858600 & -2.38887900 & 2.09244900 \\ \text { C } & 1.98771600 & -2.68633400 & 2.98326000 \\ \text { C } & 1.50566800 & -3.65733800 & 3.85944500 \\ \text { C } & -0.72341300 & -3.35416400 & 2.97414400 \\ H & -0.92307800 & -1.87849400 & 1.41817200 \\ \text { H } & 3.03409400 & -2.39826600 & 2.98977500 \\ \text { H } & 2.18676800 & -4.14939200 & 4.54898900 \\ \mathrm{H} & -1.78209900 & -3.60013300 & 2.97920000 \\ \mathrm{H} & -0.22640000 & -4.74810200 & 4.54370500\end{array}$

\section{TS-B (M062X)}

Zero-point correction= 0.694643 (Hartree/Particle)

Thermal correction to Energy=

0.742434

Thermal correction to Enthalpy=

0.743378

Thermal correction to Gibbs Free Energy=

0.610296

Sum of electronic and zero-point Energies=

$-2908.287680$

Sum of electronic and thermal Energies=

$-2908.239888$

Sum of electronic and thermal Enthalpies=

$-2908.238944$

Sum of electronic and thermal Free Energies=

$-2908.372027$

$\mathrm{E}(\mathrm{RM} 062 \mathrm{X})=-2908.98232238$

Imaginary frequency $=213 \mathrm{i}$

Relative energy $(M 062 X / 6-311+G(d, p))=-2909.72473370$
01
C
$-4.22103600$
1.87406500
0.48030500
$\mathrm{C}$
$-2.79270400$
0.37196700
$-1.34107200$
$\mathrm{C}$
$-2.86749300$
2.14782500
0.29280500
$\mathrm{C}$
$-4.87086800$
0.85417900
$-0.19744100$ 


\begin{tabular}{|c|c|c|c|}
\hline $\mathrm{C}$ & -4.13021500 & 0.10144000 & -1.10536600 \\
\hline $\mathrm{C}$ & -2.13981400 & 1.39105800 & -0.62807600 \\
\hline $\mathrm{H}$ & -2.38930500 & 2.93236200 & 0.86162300 \\
\hline $\mathrm{H}$ & -5.91962300 & 0.64603700 & -0.02099900 \\
\hline $\mathrm{H}$ & -2.22761400 & -0.22419300 & -2.05111000 \\
\hline $\mathrm{N}$ & -0.75986300 & 1.48655900 & -0.85981000 \\
\hline $\mathrm{H}$ & -0.38450900 & 0.66026800 & -1.33394300 \\
\hline $\mathrm{C}$ & 0.16048000 & 2.41705200 & -0.48102800 \\
\hline$S$ & -0.19021600 & 3.93634100 & 0.15212200 \\
\hline $\mathrm{N}$ & 1.43450900 & 1.97240100 & -0.66985400 \\
\hline $\mathrm{H}$ & 1.58630900 & 1.07344100 & -1.13967100 \\
\hline $\mathrm{C}$ & 2.59186600 & 2.84557600 & -0.62098800 \\
\hline $\mathrm{C}$ & 4.96042900 & 3.14866200 & 0.13979700 \\
\hline $\mathrm{C}$ & 4.15665700 & 4.30396500 & -1.96115900 \\
\hline $\mathrm{C}$ & 5.34706100 & 3.65990200 & -1.25280700 \\
\hline $\mathrm{C}$ & 2.97751500 & 3.33505100 & -2.02218200 \\
\hline $\mathrm{C}$ & 3.79715900 & 2.15917400 & 0.03741800 \\
\hline $\mathrm{H}$ & 4.67318300 & 3.99757900 & 0.77491200 \\
\hline $\mathrm{H}$ & 3.85308700 & 5.20673900 & -1.41554000 \\
\hline $\mathrm{H}$ & 5.71179900 & 2.81459700 & -1.85114500 \\
\hline $\mathrm{H}$ & 3.22538900 & 2.46382000 & -2.64333900 \\
\hline $\mathrm{H}$ & 4.08973300 & 1.31023700 & -0.59033800 \\
\hline $\mathrm{H}$ & 2.30400000 & 3.71782900 & -0.02587200 \\
\hline $\mathrm{H}$ & 5.83131500 & 2.67425200 & 0.60084000 \\
\hline $\mathrm{H}$ & 4.43925400 & 4.62133600 & -2.96900700 \\
\hline $\mathrm{H}$ & 6.17606700 & 4.36782900 & -1.16309400 \\
\hline $\mathrm{H}$ & 2.09689600 & 3.81454800 & -2.46039900 \\
\hline $\mathrm{C}$ & 0.37531600 & -2.11645100 & -1.28553700 \\
\hline $\mathrm{O}$ & -0.00931700 & -1.06830200 & -1.80716100 \\
\hline $\mathrm{O}$ & -0.38796300 & -3.20957600 & -1.24205500 \\
\hline $\mathrm{C}$ & -1.72669200 & -3.07339700 & -1.73272200 \\
\hline $\mathrm{H}$ & -2.23016700 & -2.28847800 & -1.16103900 \\
\hline $\mathrm{H}$ & -1.69976500 & -2.76558100 & -2.78263200 \\
\hline $\mathrm{C}$ & -2.41745500 & -4.40243600 & -1.53444800 \\
\hline $\mathrm{H}$ & -2.41623700 & -4.66707600 & -0.47415500 \\
\hline $\mathrm{H}$ & -1.91014500 & -5.19272800 & -2.09175600 \\
\hline $\mathrm{H}$ & -3.45123100 & -4.32927100 & -1.87893300 \\
\hline $\mathrm{C}$ & 1.62729900 & -2.27226500 & -0.56681000 \\
\hline $\mathrm{N}$ & 1.86950700 & -3.53138800 & -0.00218300 \\
\hline $\mathrm{C}$ & 2.78288300 & -1.49204800 & -0.97916800 \\
\hline
\end{tabular}




\begin{tabular}{|c|c|c|c|}
\hline $\mathrm{O}$ & 3.93486800 & -2.10889100 & -0.66046800 \\
\hline $\mathrm{C}$ & 5.14901000 & -1.47362800 & -1.05452200 \\
\hline $\mathrm{H}$ & 5.14882900 & -1.34185400 & -2.14119600 \\
\hline $\mathrm{H}$ & 5.20365900 & -0.47521000 & -0.60709600 \\
\hline $\mathrm{C}$ & 6.27970700 & -2.36029900 & -0.57952600 \\
\hline $\mathrm{H}$ & 6.20770100 & -3.34723000 & -1.04075100 \\
\hline $\mathrm{H}$ & 6.23050400 & -2.48895500 & 0.50435600 \\
\hline $\mathrm{H}$ & 7.24393900 & -1.91892100 & -0.84262300 \\
\hline $\mathrm{O}$ & 2.78026200 & -0.37069200 & -1.47976500 \\
\hline $\mathrm{C}$ & 2.71797400 & -4.02040600 & 0.69898700 \\
\hline $\mathrm{O}$ & 3.47877100 & -4.62135600 & 1.36479900 \\
\hline $\mathrm{N}$ & 3.43680300 & 1.53534200 & 1.36134400 \\
\hline $\mathrm{H}$ & 2.83889700 & 0.60673900 & 1.23363800 \\
\hline $\mathrm{C}$ & 2.63628100 & 2.39345300 & 2.27078100 \\
\hline $\mathrm{H}$ & 2.39970800 & 1.79833500 & 3.15385600 \\
\hline $\mathrm{H}$ & 1.70924500 & 2.69811600 & 1.78458200 \\
\hline $\mathrm{H}$ & 3.21409500 & 3.27446100 & 2.55969500 \\
\hline $\mathrm{C}$ & 4.61354400 & 0.99112700 & 2.08535900 \\
\hline $\mathrm{H}$ & 4.23834500 & 0.29762700 & 2.83800900 \\
\hline $\mathrm{H}$ & 5.18141700 & 1.79622700 & 2.55387700 \\
\hline $\mathrm{H}$ & 5.24573200 & 0.44383800 & 1.38443000 \\
\hline $\mathrm{C}$ & -4.98954400 & 2.74405500 & 1.43736600 \\
\hline $\mathrm{C}$ & -4.77623300 & -1.08174500 & -1.76591500 \\
\hline$F$ & -5.28246800 & 3.93215700 & 0.89145300 \\
\hline $\mathrm{F}$ & -4.28774300 & 2.98743700 & 2.55260400 \\
\hline $\mathrm{F}$ & -6.14892500 & 2.17774900 & 1.80189900 \\
\hline $\mathrm{F}$ & -6.04721600 & -0.83804800 & -2.10392800 \\
\hline $\mathrm{F}$ & -4.79188400 & -2.14456800 & -0.93473700 \\
\hline $\mathrm{F}$ & -4.12341600 & -1.46842600 & -2.87186600 \\
\hline $\mathrm{C}$ & 1.15601400 & -0.94417700 & 1.10882600 \\
\hline $\mathrm{O}$ & 2.26391700 & -0.67664600 & 1.66253600 \\
\hline $\mathrm{H}$ & 0.73045800 & -0.23052200 & 0.38988200 \\
\hline $\mathrm{C}$ & 0.15726800 & -1.81263000 & 1.76797900 \\
\hline $\mathrm{C}$ & -1.75522800 & -3.46621700 & 2.92799500 \\
\hline $\mathrm{C}$ & -1.17419800 & -1.74601500 & 1.35140800 \\
\hline $\mathrm{C}$ & 0.52986900 & -2.69941600 & 2.77898800 \\
\hline $\mathrm{C}$ & -0.42882700 & -3.52550800 & 3.35521200 \\
\hline $\mathrm{C}$ & -2.13191100 & -2.57179100 & 1.92662800 \\
\hline $\mathrm{H}$ & -1.45210200 & -1.03960400 & 0.57099200 \\
\hline $\mathrm{H}$ & 1.56790000 & -2.72886100 & 3.09685400 \\
\hline
\end{tabular}




$\begin{array}{llll}\mathrm{H} & -0.14294900 & -4.22130800 & 4.13744500 \\ \mathrm{H} & -3.16352100 & -2.51555300 & 1.59091500 \\ \mathrm{H} & -2.49767200 & -4.11647100 & 3.37987600\end{array}$

\section{TS-B (APFD)}

Zero-point correction $=$ 0.690351 (Hartree/Particle)

Thermal correction to Energy=

0.738588

Thermal correction to Enthalpy=

0.739533

Thermal correction to Gibbs Free Energy=

0.604286

Sum of electronic and zero-point Energies= $-2907.327943$

Sum of electronic and thermal Energies=

$-2907.279705$

Sum of electronic and thermal Enthalpies=

$-2907.278761$

Sum of electronic and thermal Free Energies=

$-2907.414008$

$\mathrm{E}(\mathrm{RAPFD})=-2908.01829384$

Imaginary frequency $=250 \mathrm{i}$

Relative energy $(A P F D / 6-311+G(d, p))=-2908.67803015$

$\begin{array}{crrr}01 & & & \\ \mathrm{C} & -3.68240700 & 2.01695300 & 0.61999400 \\ \mathrm{C} & -2.66233200 & 0.74602600 & -1.61758600 \\ \mathrm{C} & -2.34988700 & 2.21327300 & 0.27509000 \\ \mathrm{C} & -4.51205700 & 1.17863600 & -0.11311700 \\ \mathrm{C} & -3.97966100 & 0.54237100 & -1.23076900 \\ \mathrm{C} & -1.82554600 & 1.56892100 & -0.84911600 \\ \mathrm{H} & -1.71684100 & 2.84390300 & 0.88468400 \\ \mathrm{H} & -5.54148900 & 1.01497200 & 0.18445900 \\ \mathrm{H} & -2.25873200 & 0.22864000 & -2.48075200 \\ \mathrm{~N} & -0.47304000 & 1.56806100 & -1.19743400 \\ \mathrm{H} & -0.18951900 & 0.69711900 & -1.66246800 \\ \mathrm{C} & 0.54169900 & 2.37867700 & -0.76821300 \\ \mathrm{~S} & 0.34047700 & 3.94735800 & -0.18454300 \\ \mathrm{~N} & 1.74859400 & 1.76897600 & -0.87516300 \\ \mathrm{H} & 1.80925800 & 0.82750300 & -1.28257000 \\ \mathrm{C} & 3.01162400 & 2.45880500 & -0.76233000 \\ \mathrm{C} & 5.31403800 & 2.47290700 & 0.22520900 \\ \mathrm{C} & 4.92628700 & 3.48380400 & -2.05559200 \\ \mathrm{C} & 5.91473500 & 2.74433600 & -1.15696500 \\ \mathrm{C} & 3.61075000 & 2.71563200 & -2.15065800\end{array}$


$\mathrm{H}$

$\mathrm{H}$

$\mathrm{H}$

H

$\mathrm{H}$

C

O

O

C

H

$\mathrm{H}$

C

H

H

$\mathrm{H}$

C

N

$\mathrm{H}$

$\mathrm{H}$

H

O

C

$\mathrm{O}$

$\begin{array}{lll}4.01403700 & 1.67375200 & 0.09670300\end{array}$

$\begin{array}{lll}5.11427400 & 3.42684200 & 0.73202900\end{array}$

$\begin{array}{lll}4.73463400 & 4.48419000 & -1.64334400\end{array}$

$\begin{array}{lll}6.18503200 & 1.78618000 & -1.62325900\end{array}$

$\begin{array}{lll}3.76527000 & 1.75033600 & -2.65182300\end{array}$

$\begin{array}{lll}4.21648200 & 0.72828700 & -0.41792300\end{array}$

$\begin{array}{lll}2.79904300 & 3.42585800 & -0.29182000\end{array}$

$\begin{array}{lll}6.04349000 & 1.93042600 & 0.83516700\end{array}$

$\begin{array}{lll}5.35248300 & 3.63366000 & -3.05395200\end{array}$

$\begin{array}{lll}6.84551900 & 3.31223500 & -1.04786400\end{array}$

$2.87062900 \quad 3.27059100 \quad-2.73693100$

$\begin{array}{lll}0.10448000 & -2.07827600 & -1.35261100\end{array}$

$\begin{array}{lll}-0.09553600 & -1.05827300 & -2.01591600\end{array}$

$\begin{array}{lll}-0.76858300 & -3.08353300 & -1.29022600\end{array}$

$\begin{array}{lll}-2.03225200 & -2.87624800 & -1.93617300\end{array}$

$\begin{array}{lll}-2.46466600 & -1.94763600 & -1.56243000\end{array}$

$\begin{array}{lll}-1.87265900 & -2.76264500 & -3.01355500\end{array}$

$\begin{array}{lll}-2.90756900 & -4.05847900 & -1.59730300\end{array}$

$\begin{array}{lll}-3.02622700 & -4.13973500 & -0.51336400\end{array}$

$\begin{array}{lll}-2.47438700 & -4.99109100 & -1.96905800\end{array}$

$\begin{array}{lll}-3.89565300 & -3.92519100 & -2.04543800\end{array}$

$\begin{array}{lll}1.25024100 & -2.28789300 & -0.47365300\end{array}$

$1.28865000 \quad-3.51151200 \quad 0.19781200$

$\begin{array}{lll}2.53264000 & -1.72636300 & -0.89387800\end{array}$

$\begin{array}{lll}3.56177300 & -2.43556200 & -0.39856800\end{array}$

$\begin{array}{lll}4.87752900 & -2.06542800 & -0.81242200\end{array}$

$\begin{array}{lll}4.90870900 & -2.01617100 & -1.90591300\end{array}$

$\begin{array}{lll}5.11455700 & -1.06303100 & -0.43884300\end{array}$

$\begin{array}{lll}5.82109500 & -3.10484800 & -0.25046300\end{array}$

$\begin{array}{lll}5.58090300 & -4.09640800 & -0.64272800\end{array}$

$\begin{array}{lll}5.74556600 & -3.14767800 & 0.83980300\end{array}$

$6.85405200 \quad-2.86603700 \quad-0.52085300$

$\begin{array}{lll}2.71288900 & -0.70790800 & -1.56107400\end{array}$

$\begin{array}{lll}1.95648700 & -4.00659900 & 1.07265100\end{array}$

$\begin{array}{lll}2.53356100 & -4.61947800 & 1.89817900\end{array}$

$\begin{array}{lll}3.43808800 & 1.25757400 & 1.42031500\end{array}$

$\begin{array}{lll}2.65334800 & 0.42771800 & 1.31375600\end{array}$

$\begin{array}{lll}2.78228900 & 2.33774300 & 2.18806800\end{array}$

$\begin{array}{lll}2.34229300 & 1.89068500 & 3.08091600\end{array}$

$\begin{array}{lll}1.98957300 & 2.79753300 & 1.59537900\end{array}$ 


\begin{tabular}{lrrr}
$\mathrm{H}$ & 3.51251500 & 3.09639600 & 2.47972300 \\
$\mathrm{C}$ & 4.42168900 & 0.55114100 & 2.27224800 \\
$\mathrm{H}$ & 3.86644500 & 0.03096100 & 3.05300500 \\
$\mathrm{H}$ & 5.13137200 & 1.24928300 & 2.71918900 \\
$\mathrm{H}$ & 4.95194200 & -0.19132500 & 1.67441600 \\
$\mathrm{C}$ & -4.23860900 & 2.75395300 & 1.80973800 \\
$\mathrm{C}$ & -4.83534000 & -0.45942700 & -1.95269900 \\
$\mathrm{~F}$ & -5.25851600 & 2.08123000 & 2.37730400 \\
$\mathrm{~F}$ & -4.71046700 & 3.96532600 & 1.45852600 \\
$\mathrm{~F}$ & -3.30413900 & 2.95081600 & 2.75672900 \\
$\mathrm{~F}$ & -6.07405700 & 0.00570800 & -2.18359900 \\
$\mathrm{~F}$ & -4.97674700 & -1.58560700 & -1.20754000 \\
$\mathrm{~F}$ & -4.30977100 & -0.83791400 & -3.13129700 \\
$\mathrm{C}$ & 0.78847900 & -0.83609000 & 1.04272400 \\
$\mathrm{O}$ & 1.87645900 & -0.65716000 & 1.68825000 \\
$\mathrm{H}$ & 0.50236700 & -0.10599800 & 0.27983100 \\
$\mathrm{C}$ & -0.34941700 & -1.52814200 & 1.67572500 \\
$\mathrm{C}$ & -2.52037100 & -2.86418700 & 2.80480800 \\
$\mathrm{C}$ & -1.63015400 & -1.37627900 & 1.13633700 \\
$\mathrm{C}$ & -0.16086300 & -2.34166400 & 2.79617400 \\
$\mathrm{C}$ & -1.24564300 & -3.00944400 & 3.35530000 \\
$\mathrm{C}$ & -2.71473500 & -2.04053700 & 1.69495600 \\
$\mathrm{H}$ & -1.77371700 & -0.73133000 & 0.27610200 \\
$\mathrm{H}$ & 0.83724300 & -2.43787400 & 3.21332200 \\
$\mathrm{H}$ & -1.09899200 & -3.64847100 & 4.22202100 \\
$\mathrm{H}$ & -3.70274400 & -1.91548900 & 1.26030000 \\
& -3.36384400 & -3.38946400 & 3.24544400 \\
\hline & & & \\
\hline & &
\end{tabular}

\section{TS-B $(\omega B$ 97XD)}

Zero-point correction= 0.696395 (Hartree/Particle)

Thermal correction to Energy=

0.744171

Thermal correction to Enthalpy=

0.745115

Thermal correction to Gibbs Free Energy=

0.612787

Sum of electronic and zero-point Energies=

$-2908.562800$

Sum of electronic and thermal Energies=

$-2908.515025$

Sum of electronic and thermal Enthalpies=

$-2908.514080$

Sum of electronic and thermal Free Energies=

$-2908.646408$

$\mathrm{E}(\omega \mathrm{B} 97 \mathrm{XD})=-2909.25919565$

Imaginary frequency $=271 \mathrm{i}$ 
Relative energy $(\omega B$ 97XD/6-311+G(d,p) $)=-2909.92493989$

01

C

C

$\mathrm{C}$

$\mathrm{C}$

C

$\mathrm{C}$

$\mathrm{H}$

$\mathrm{H}$

$\mathrm{H}$

$\mathrm{N}$

$\mathrm{H}$

$\mathrm{C}$

S

$\mathrm{N}$

$\mathrm{H}$

$\mathrm{C}$

C

$\mathrm{C}$

C

C

C

$\mathrm{H}$

$\mathrm{H}$

$\mathrm{H}$

$\mathrm{H}$

$\mathrm{H}$

$\mathrm{H}$

$\mathrm{H}$

$\mathrm{H}$

$\mathrm{H}$

H

C

$\mathrm{O}$

O

C

$\mathrm{H}$

$\mathrm{H}$

\begin{tabular}{rrr}
3.95526500 & -1.92418700 & 0.57598400 \\
2.73557700 & -0.59138000 & -1.51638000 \\
2.60582700 & -2.14699600 & 0.32199300 \\
4.70147100 & -1.03239600 & -0.18128500 \\
4.07162900 & -0.36279300 & -1.22527000 \\
1.98526700 & -1.47695900 & -0.73262500 \\
2.03854400 & -2.82422000 & 0.94380000 \\
5.74641900 & -0.85450900 & 0.04107100 \\
2.24948700 & -0.05224600 & -2.32135400 \\
0.61035100 & -1.52196000 & -1.01131000 \\
0.27733800 & -0.67071400 & -1.47416300 \\
-0.35252800 & -2.39826500 & -0.60761500 \\
-0.06743900 & -3.94183500 & 0.00509800 \\
-1.60061200 & -1.88243500 & -0.75767800 \\
-1.71617700 & -0.96143100 & -1.19217400 \\
-2.81189800 & -2.67189600 & -0.68660300 \\
-5.17526400 & -2.81417800 & 0.15289800 \\
-4.55638700 & -3.91145800 & -2.04068200 \\
-5.65674700 & -3.20756000 & -1.24850500 \\
-3.29613900 & -3.05065400 & -2.09313300 \\
-3.92898400 & -1.92885800 & 0.06384100 \\
-4.94760600 & -3.72086200 & 0.72839300 \\
-4.32151600 & -4.87215000 & -1.56507700 \\
-5.96818500 & -2.30117200 & -1.78399000 \\
-3.48541400 & -2.12946300 & -2.65914500 \\
-4.16320600 & -1.02241300 & -0.50322400 \\
-2.56048600 & -3.59328000 & -0.15318900 \\
-5.98335600 & -2.29243400 & 0.67283900 \\
-4.90200000 & -4.13556300 & -3.05413300 \\
-6.54205300 & -3.84440400 & -1.16218000 \\
-2.48047600 & -3.58075400 & -2.59317900 \\
\hline. .24854400 & 2.10939200 & -1.31216500 \\
\hline .5604515900 & 1.08216900 & -1.92397200 \\
\hline & 3.16416600 & -1.26248000 \\
\hline
\end{tabular}




\begin{tabular}{|c|c|c|c|}
\hline $\mathrm{C}$ & 2.64566500 & 4.26482300 & -1.53095600 \\
\hline $\mathrm{H}$ & 2.72050500 & 4.40524500 & -0.44974900 \\
\hline $\mathrm{H}$ & 2.16524400 & 5.14445200 & -1.96572000 \\
\hline $\mathrm{H}$ & 3.65335000 & 4.17216500 & -1.94263000 \\
\hline $\mathrm{C}$ & -1.44217500 & 2.27330400 & -0.48580500 \\
\hline $\mathrm{N}$ & -1.58830600 & 3.53428100 & 0.11616100 \\
\hline $\mathrm{C}$ & -2.67225100 & 1.62394800 & -0.93715600 \\
\hline $\mathrm{O}$ & -3.76346400 & 2.30905200 & -0.56517100 \\
\hline $\mathrm{C}$ & -5.03666900 & 1.79780700 & -0.95605800 \\
\hline $\mathrm{H}$ & -5.05982900 & 1.67269700 & -2.04262700 \\
\hline $\mathrm{H}$ & -5.18832400 & 0.80843100 & -0.51112400 \\
\hline $\mathrm{C}$ & -6.07596800 & 2.78369300 & -0.46718700 \\
\hline $\mathrm{H}$ & -5.93249700 & 3.75866400 & -0.93792900 \\
\hline $\mathrm{H}$ & -5.99959800 & 2.91509400 & 0.61496000 \\
\hline $\mathrm{H}$ & -7.07905900 & 2.42295900 & -0.71011000 \\
\hline $\mathrm{O}$ & -2.76396300 & 0.54961300 & -1.52482100 \\
\hline $\mathrm{C}$ & -2.33758600 & 4.04707600 & 0.90693100 \\
\hline $\mathrm{O}$ & -2.99888000 & 4.67567400 & 1.65195100 \\
\hline $\mathrm{N}$ & -3.47431000 & -1.41212500 & 1.40547600 \\
\hline $\mathrm{H}$ & -2.77832100 & -0.56928700 & 1.28812300 \\
\hline $\mathrm{C}$ & -2.77048100 & -2.39656600 & 2.26340200 \\
\hline $\mathrm{H}$ & -2.45445100 & -1.87719500 & 3.16839700 \\
\hline $\mathrm{H}$ & -1.88974000 & -2.78440700 & 1.75110200 \\
\hline $\mathrm{H}$ & -3.44254800 & -3.21715900 & 2.52212400 \\
\hline $\mathrm{C}$ & -4.56365200 & -0.75095500 & 2.16707200 \\
\hline $\mathrm{H}$ & -4.09850600 & -0.14693300 & 2.94533300 \\
\hline $\mathrm{H}$ & -5.23324400 & -1.49064400 & 2.60701300 \\
\hline $\mathrm{H}$ & -5.11862200 & -0.09137400 & 1.49955300 \\
\hline $\mathrm{C}$ & 4.62589600 & -2.69344400 & 1.68483800 \\
\hline $\mathrm{C}$ & 4.83696700 & 0.69180700 & -1.97551500 \\
\hline $\mathrm{F}$ & 3.77616100 & -2.97048500 & 2.68522800 \\
\hline $\mathrm{F}$ & 5.65777600 & -2.00899700 & 2.20778200 \\
\hline $\mathrm{F}$ & 5.11375500 & -3.86413500 & 1.24200900 \\
\hline $\mathrm{F}$ & 6.08889000 & 0.30275400 & -2.25511000 \\
\hline $\mathrm{F}$ & 4.93680600 & 1.82135200 & -1.23880100 \\
\hline $\mathrm{F}$ & 4.24575200 & 1.03297300 & -3.13080800 \\
\hline $\mathrm{C}$ & -0.97700900 & 0.91359400 & 1.09250600 \\
\hline $\mathrm{O}$ & -2.06672500 & 0.68137900 & 1.70498400 \\
\hline $\mathrm{H}$ & -0.61592700 & 0.18013700 & 0.36303200 \\
\hline $\mathrm{C}$ & 0.11414600 & 1.67471100 & 1.75261100 \\
\hline
\end{tabular}




$\begin{array}{lrrr}\mathrm{C} & 2.19320600 & 3.10879300 & 2.93100300 \\ \mathrm{C} & 1.42153200 & 1.53805800 & 1.28292300 \\ \mathrm{C} & -0.14624000 & 2.52086700 & 2.83024800 \\ \mathrm{C} & 0.89200800 & 3.23796000 & 3.41380500 \\ \mathrm{C} & 2.46040400 & 2.25160500 & 1.86572200 \\ \mathrm{H} & 1.62512300 & 0.86574200 & 0.45541600 \\ \mathrm{H} & -1.16326200 & 2.60797100 & 3.19842200 \\ \mathrm{H} & 0.68646700 & 3.90386400 & 4.24581800 \\ \mathrm{H} & 3.47017300 & 2.13681900 & 1.48381700 \\ \mathrm{H} & 3.00017600 & 3.67121400 & 3.39008600\end{array}$

\section{TS-C (B3LYP)}

Zero-point correction= 0.687451 (Hartree/Particle)

Thermal correction to Energy=

0.736675

Thermal correction to Enthalpy=

0.737619

Thermal correction to Gibbs Free Energy=

0.598166

Sum of electronic and zero-point Energies=

$-2909.224519$

Sum of electronic and thermal Energies=

$-2909.175296$

Sum of electronic and thermal Enthalpies=

$-2909.174352$

Sum of electronic and thermal Free Energies=

$-2909.313805$

$\mathrm{E}(\mathrm{RB} 3 \mathrm{LYP})=-2909.91197058$

Imaginary frequency $=168 \mathrm{i}$

Relative energy $(B 3 L Y P / 6-311+G(d, p))=-2910.67581149$

01

$\mathrm{C}$

$\mathrm{C}$

5.47620400

0.74083700

$-0.26013500$

C

3.34645100

$-0.81674300$

0.56786900

$\mathrm{C}$

4.20903900

1.31786000

$-0.16275400$

$\mathrm{C}$

5.69957700

$-0.60421100$

0.03364000

C

4.61455400

$-1.37569600$

0.44603600

C

3.12089700

0.53458200

0.25478900

$\mathrm{H}$

4.06496100

2.36225500

$-0.39519100$

$\mathrm{H}$

6.69021500

$-1.03439800$

$-0.04636900$

$\mathrm{H}$

2.51494400

$-1.42395600$

0.90513400

$\mathrm{N}$

1.78629400

0.96090300

0.37611400

$\mathrm{H}$

1.14170400

0.18333600

0.53561400

C

1.19168700

2.19007300

0.29970500

$\mathrm{S}$

1.99597200

3.66059600

0.03391500

$\mathrm{N}$

$-0.16521400$

2.11526200

0.44665100

$\mathrm{H}$

$-0.60267200$

1.21910000

0.68321200 


\begin{tabular}{|c|c|c|c|}
\hline $\mathrm{C}$ & -1.01714800 & 3.28842100 & 0.54856200 \\
\hline $\mathrm{C}$ & -3.12079500 & 4.45658200 & -0.19452100 \\
\hline $\mathrm{C}$ & -2.15077500 & 4.94495400 & 2.10738500 \\
\hline $\mathrm{C}$ & -3.42584200 & 4.85683200 & 1.26092500 \\
\hline C & -1.33078900 & 3.65115600 & 2.01557400 \\
\hline $\mathrm{C}$ & -2.34044500 & 3.13348300 & -0.23275800 \\
\hline $\mathrm{H}$ & -2.53374800 & 5.24965000 & -0.67789200 \\
\hline $\mathrm{H}$ & -1.53743800 & 5.78704100 & 1.75704100 \\
\hline $\mathrm{H}$ & -4.10589100 & 4.11179700 & 1.69843400 \\
\hline $\mathrm{H}$ & -1.86598000 & 2.81423800 & 2.48322100 \\
\hline $\mathrm{H}$ & -2.93726700 & 2.35025800 & 0.24740600 \\
\hline $\mathrm{H}$ & -0.44529800 & 4.11615700 & 0.11924200 \\
\hline $\mathrm{H}$ & -4.06315500 & 4.37014500 & -0.74560900 \\
\hline $\mathrm{H}$ & -2.40529700 & 5.15785700 & 3.15245200 \\
\hline $\mathrm{H}$ & -3.96364500 & 5.81216200 & 1.26744900 \\
\hline $\mathrm{H}$ & -0.37667500 & 3.76208800 & 2.54108200 \\
\hline $\mathrm{C}$ & -0.64730100 & -2.20132700 & 0.44104100 \\
\hline $\mathrm{O}$ & 0.21142900 & -1.40675400 & 0.80221900 \\
\hline $\mathrm{O}$ & -0.34865100 & -3.40212000 & -0.08773700 \\
\hline $\mathrm{C}$ & 1.05428100 & -3.74205500 & -0.21633000 \\
\hline $\mathrm{H}$ & 1.54782000 & -2.97654600 & -0.82132800 \\
\hline $\mathrm{H}$ & 1.50952700 & -3.73402600 & 0.77848000 \\
\hline $\mathrm{C}$ & 1.13791600 & -5.11194600 & -0.85988200 \\
\hline $\mathrm{H}$ & 0.68448600 & -5.10713700 & -1.85603900 \\
\hline $\mathrm{H}$ & 0.62586700 & -5.86400400 & -0.25183500 \\
\hline $\mathrm{H}$ & 2.19088200 & -5.39570700 & -0.95623000 \\
\hline $\mathrm{C}$ & -2.11355300 & -1.95974300 & 0.45359700 \\
\hline $\mathrm{N}$ & -2.92033700 & -3.11852800 & 0.41400900 \\
\hline $\mathrm{C}$ & -2.57095200 & -0.94162600 & 1.43200700 \\
\hline $\mathrm{O}$ & -3.69194700 & -1.31015400 & 2.08359000 \\
\hline $\mathrm{C}$ & -4.23762900 & -0.35568600 & 3.01493400 \\
\hline $\mathrm{H}$ & -3.47087400 & -0.09918000 & 3.75320500 \\
\hline $\mathrm{H}$ & -4.49714900 & 0.56331300 & 2.47648000 \\
\hline $\mathrm{C}$ & -5.45650900 & -0.99626800 & 3.65270700 \\
\hline $\mathrm{H}$ & -5.18086200 & -1.91262500 & 4.18357200 \\
\hline $\mathrm{H}$ & -6.20246800 & -1.25097200 & 2.89343400 \\
\hline $\mathrm{H}$ & -5.91041700 & -0.30342100 & 4.36987900 \\
\hline $\mathrm{O}$ & -2.04692900 & 0.15479400 & 1.60016200 \\
\hline $\mathrm{C}$ & -2.93343100 & -4.20753100 & -0.10494900 \\
\hline $\mathrm{O}$ & -3.08105300 & -5.29838200 & -0.53626500 \\
\hline
\end{tabular}




\begin{tabular}{lrrr} 
N & -2.12257400 & 2.58581900 & -1.64197600 \\
$\mathrm{H}$ & -1.91101000 & 1.52173500 & -1.49318900 \\
$\mathrm{C}$ & -1.00456100 & 3.20440600 & -2.41560500 \\
$\mathrm{H}$ & -0.90259900 & 2.64885700 & -3.35007600 \\
$\mathrm{H}$ & -0.07148600 & 3.14720500 & -1.85898200 \\
$\mathrm{H}$ & -1.24227600 & 4.24835500 & -2.63557400 \\
$\mathrm{C}$ & -3.36049600 & 2.58492200 & -2.47641000 \\
$\mathrm{H}$ & -3.18421400 & 1.92519100 & -3.32803500 \\
$\mathrm{H}$ & -3.58247500 & 3.59359000 & -2.82845200 \\
$\mathrm{H}$ & -4.19447100 & 2.18877600 & -1.89900100 \\
$\mathrm{C}$ & 6.62491300 & 1.57601700 & -0.76548100 \\
$\mathrm{C}$ & 4.77213100 & -2.84747900 & 0.71294800 \\
$\mathrm{~F}$ & 7.79515000 & 1.21309100 & -0.19148200 \\
$\mathrm{~F}$ & 6.44606900 & 2.89087800 & -0.52715800 \\
$\mathrm{~F}$ & 6.78858700 & 1.43454800 & -2.10285900 \\
$\mathrm{~F}$ & 6.05837100 & -3.20611200 & 0.89126100 \\
$\mathrm{~F}$ & -4.29310200 & -3.59167300 & -0.32195700 \\
$\mathrm{~F}$ & -4.08096500 & -3.24120600 & 1.80861000 \\
$\mathrm{C}$ & -2.30752000 & -1.05159500 & -1.30848200 \\
$\mathrm{O}$ & -1.64487500 & 0.05195300 & -1.32318800 \\
$\mathrm{H}$ & -1.84562400 & -1.90732700 & -1.83577600 \\
$\mathrm{C}$ & -3.80737100 & -1.05814200 & -1.51603100 \\
$\mathrm{C}$ & -6.58133900 & -1.07916700 & -1.99809100 \\
$\mathrm{C}$ & -4.37221700 & -1.97014600 & -2.41528100 \\
$\mathrm{C}$ & -4.65753100 & -0.16064900 & -0.85404000 \\
$\mathrm{H}$ & -6.03158700 & -0.17075100 & -1.08832500 \\
$\mathrm{H}$ & -5.74739800 & -1.97991100 & -2.66001700 \\
$\mathrm{H}$ & -3.72729100 & -2.67700500 & -2.93224200 \\
$\mathrm{H}$ & -4.23576100 & 0.53143100 & -0.13123300 \\
$\mathrm{H}$ & -6.67839800 & 0.52251800 & -0.55564600 \\
$\mathrm{H}$ & -2.69397700 & -3.36467700 \\
$\mathrm{H}$ & -1.08764800 & -2.18190500 \\
\hline
\end{tabular}

\section{TS-C (M062X)}

Zero-point correction=

0.694216 (Hartree/Particle)

Thermal correction to Energy=

0.742296

Thermal correction to Enthalpy=

0.743240

Thermal correction to Gibbs Free Energy=

0.609274

Sum of electronic and zero-point Energies=

$-2908.283730$

Sum of electronic and thermal Energies=

$-2908.235651$ 
$\mathrm{E}(\mathrm{RM} 062 \mathrm{X})=-2908.97794657$

Imaginary frequency $=133 \mathrm{i}$

Relative energy (M062X/6-311+G(d,p)) = -2909.72176149

01

$\mathrm{C}$

$\mathrm{C}$

C

$\mathrm{C}$

$\mathrm{C}$

$\mathrm{C}$

$\mathrm{H}$

$\mathrm{H}$

$\mathrm{H}$

$\mathrm{N}$

$\mathrm{H}$

C

S

$\mathrm{N}$

$\mathrm{H}$

C

C

C

C

C

C

$\mathrm{H}$

$\mathrm{H}$

$\mathrm{H}$

$\mathrm{H}$

H

$\mathrm{H}$

$\mathrm{H}$

$\mathrm{H}$

$\mathrm{H}$

$\mathrm{H}$

$\begin{array}{rrr}5.34892800 & 0.80983400 & -0.47433100 \\ 3.33183700 & -0.69715600 & 0.64132000 \\ 4.07233600 & 1.35264800 & -0.39445100 \\ 5.64192900 & -0.47715900 & -0.03056900 \\ 4.61083000 & -1.22214000 & 0.52261200 \\ 3.04457200 & 0.58833000 & 0.17353500 \\ 3.87743200 & 2.34993600 & -0.76116700 \\ 6.64379400 & -0.88306800 & -0.11466600 \\ 2.53708700 & -1.27668500 & 1.10129600 \\ 1.69983000 & 0.96276100 & 0.27106900 \\ 1.07785900 & 0.16710100 & 0.42334600 \\ 1.09658500 & 2.17707300 & 0.33895900 \\ 1.87595500 & 3.66426600 & 0.16877900 \\ -0.24050200 & 2.05845800 & 0.54114600 \\ -0.64070000 & 1.13794500 & 0.75514500 \\ -1.11976700 & 3.19404300 & 0.70005100 \\ -3.25559100 & 4.29584300 & -0.00091500 \\ -2.29229400 & 4.74613300 & 2.29810100 \\ -3.56751900 & 4.62708800 & 1.46455300 \\ -1.43422300 & 3.48737800 & 2.17141200 \\ -2.43445200 & 3.00875400 & -0.07056100 \\ -2.69073400 & 5.12237500 & -0.45269600 \\ -1.71227800 & 5.61194700 & 1.95277200 \\ -4.19814200 & 3.82831700 & 1.87792000 \\ -1.94671600 & 2.61497100 & 2.59767000 \\ -2.99275500 & 2.18837300 & 0.40023600 \\ -0.58922200 & 4.06053200 & 0.28934100 \\ -4.19315600 & 4.19596000 & -0.55600500 \\ -2.54311400 & 4.93161700 & 3.34661000 \\ -4.15191200 & 5.55054200 & 1.51212100 \\ -0.48209600 & 3.60734300 & 2.69633900\end{array}$




\begin{tabular}{|c|c|c|c|}
\hline $\mathrm{C}$ & -0.61806400 & -2.11963400 & 0.70501100 \\
\hline $\mathrm{O}$ & 0.16438400 & -1.23905400 & 1.03763600 \\
\hline $\mathrm{O}$ & -0.20388500 & -3.32775300 & 0.28761700 \\
\hline $\mathrm{C}$ & 1.21133100 & -3.56167700 & 0.24304700 \\
\hline $\mathrm{H}$ & 1.67128500 & -2.84199200 & -0.44237700 \\
\hline $\mathrm{H}$ & 1.63364800 & -3.39809900 & 1.24018600 \\
\hline $\mathrm{C}$ & 1.40552000 & -4.98802800 & -0.22028900 \\
\hline $\mathrm{H}$ & 0.97020300 & -5.13224800 & -1.21160900 \\
\hline $\mathrm{H}$ & 0.92215000 & -5.68211300 & 0.47055100 \\
\hline $\mathrm{H}$ & 2.47223100 & -5.21436900 & -0.26351300 \\
\hline $\mathrm{C}$ & -2.06430100 & -1.99249500 & 0.61965900 \\
\hline $\mathrm{N}$ & -2.81455500 & -3.16678400 & 0.46954700 \\
\hline $\mathrm{C}$ & -2.71323800 & -0.89284900 & 1.31600400 \\
\hline $\mathrm{O}$ & -3.97190100 & -1.18473400 & 1.68767600 \\
\hline $\mathrm{C}$ & -4.71698000 & -0.11831500 & 2.27189300 \\
\hline $\mathrm{H}$ & -4.21390800 & 0.21991000 & 3.18370400 \\
\hline $\mathrm{H}$ & -4.74085500 & 0.73335200 & 1.57898400 \\
\hline $\mathrm{C}$ & -6.10690600 & -0.65127700 & 2.54336500 \\
\hline $\mathrm{H}$ & -6.06270400 & -1.49753800 & 3.23180200 \\
\hline $\mathrm{H}$ & -6.56719700 & -0.99079200 & 1.61230500 \\
\hline $\mathrm{H}$ & -6.73209100 & 0.12813100 & 2.98567200 \\
\hline $\mathrm{O}$ & -2.23908700 & 0.22694500 & 1.47697600 \\
\hline $\mathrm{C}$ & -2.66897600 & -4.25579200 & -0.02298800 \\
\hline $\mathrm{O}$ & -2.65890700 & -5.34678300 & -0.46161300 \\
\hline $\mathrm{N}$ & -2.18032700 & 2.52276800 & -1.48032600 \\
\hline $\mathrm{H}$ & -1.89780700 & 1.46821400 & -1.36956200 \\
\hline $\mathrm{C}$ & -1.08281800 & 3.22287800 & -2.20021900 \\
\hline $\mathrm{H}$ & -0.99721700 & 2.77269300 & -3.19012400 \\
\hline $\mathrm{H}$ & -0.13923400 & 3.11473100 & -1.66995500 \\
\hline $\mathrm{H}$ & -1.33304500 & 4.28166400 & -2.30049400 \\
\hline $\mathrm{C}$ & -3.39672400 & 2.53438300 & -2.33253400 \\
\hline $\mathrm{H}$ & -3.24222200 & 1.82866600 & -3.15051300 \\
\hline $\mathrm{H}$ & -3.55939500 & 3.53849600 & -2.72835300 \\
\hline $\mathrm{H}$ & -4.26458900 & 2.21983000 & -1.75688800 \\
\hline $\mathrm{C}$ & 6.46652600 & 1.60551000 & -1.09028200 \\
\hline $\mathrm{C}$ & 4.82306700 & -2.64321000 & 0.95322000 \\
\hline $\mathrm{F}$ & 6.94025600 & 1.00072800 & -2.19246100 \\
\hline $\mathrm{F}$ & 7.50233200 & 1.72487000 & -0.24518800 \\
\hline$F$ & 6.08462800 & 2.83799400 & -1.44102200 \\
\hline $\mathrm{F}$ & 6.11720000 & -2.95262800 & 1.07947100 \\
\hline
\end{tabular}




$\begin{array}{lrrr}\text { F } & 4.29469700 & -3.50242500 & 0.05555900 \\ \text { F } & 4.21952600 & -2.90626100 & 2.12241600 \\ \text { C } & -1.98305700 & -1.07189100 & -1.36051400 \\ \text { O } & -1.42903900 & 0.05627300 & -1.26044200 \\ \text { H } & -1.36872100 & -1.92985400 & -1.68293600 \\ \text { C } & -3.43444800 & -1.22810400 & -1.66900200 \\ \text { C } & -6.15528000 & -1.53702800 & -2.22445600 \\ \text { C } & -3.87679800 & -2.30213000 & -2.44098700 \\ \text { C } & -4.36833200 & -0.32281900 & -1.16203300 \\ \text { C } & -5.72119700 & -0.47137500 & -1.43514600 \\ \text { C } & -5.23261000 & -2.45213800 & -2.72322500 \\ H & -3.15657300 & -3.02156400 & -2.82281900 \\ \text { H } & -4.01479100 & 0.47553500 & -0.51487700 \\ H & -6.44089200 & 0.23148300 & -1.02441700 \\ \text { H } & -5.56771500 & -3.28876800 & -3.32728800 \\ \text { H } & -7.21190500 & -1.65898700 & -2.43947500\end{array}$

\section{TS-C (APFD)}

Zero-point correction= 0.690830 (Hartree/Particle)

Thermal correction to Energy=

0.739093

Thermal correction to Enthalpy=

0.740037

Thermal correction to Gibbs Free Energy=

0.603271

Sum of electronic and zero-point Energies=

$-2907.320833$

Sum of electronic and thermal Energies=

$-2907.272570$

Sum of electronic and thermal Enthalpies=

$-2907.271625$

Sum of electronic and thermal Free Energies=

$-2907.408392$

$\mathrm{E}(\mathrm{RAPFD})=-2908.01166237$

Imaginary frequency $=122 \mathrm{i}$

Relative energy $($ APFD $/ 6-311+G(d, p))=-2908.67316883$

01

$\begin{array}{lrrr}\mathrm{C} & 5.26826300 & 0.82301700 & -0.49110400 \\ \mathrm{C} & 3.33620100 & -0.68381600 & 0.77799000 \\ \mathrm{C} & 3.98877500 & 1.35189600 & -0.34066600 \\ \mathrm{C} & 5.59927800 & -0.45104700 & -0.04470800 \\ \mathrm{C} & 4.60882300 & -1.19773500 & 0.58635600 \\ \mathrm{C} & 3.00460900 & 0.59301400 & 0.30193100 \\ \mathrm{H} & 3.75372400 & 2.33947300 & -0.71463300\end{array}$




\begin{tabular}{|c|c|c|c|}
\hline $\mathrm{H}$ & 6.59620300 & -0.85062900 & -0.18665000 \\
\hline $\mathrm{H}$ & 2.58095700 & -1.26650500 & 1.29290100 \\
\hline $\mathrm{N}$ & 1.67068100 & 0.95826500 & 0.46567300 \\
\hline $\mathrm{H}$ & 1.06027500 & 0.15790300 & 0.65488700 \\
\hline $\mathrm{C}$ & 1.04714400 & 2.16588800 & 0.45420000 \\
\hline$S$ & 1.80884200 & 3.66196900 & 0.23797900 \\
\hline $\mathrm{N}$ & -0.29148800 & 2.03572800 & 0.63354500 \\
\hline $\mathrm{H}$ & -0.69388800 & 1.11854300 & 0.85358300 \\
\hline $\mathrm{C}$ & -1.19034500 & 3.15463500 & 0.73337500 \\
\hline $\mathrm{C}$ & -3.27319600 & 4.25263600 & -0.11600400 \\
\hline $\mathrm{C}$ & -2.50590600 & 4.66140000 & 2.26329300 \\
\hline $\mathrm{C}$ & -3.70710100 & 4.54798900 & 1.32465500 \\
\hline $\mathrm{C}$ & -1.62467600 & 3.41666400 & 2.17934000 \\
\hline $\mathrm{C}$ & -2.43753000 & 2.97291200 & -0.14621600 \\
\hline $\mathrm{H}$ & -2.68296700 & 5.09319100 & -0.50537000 \\
\hline $\mathrm{H}$ & -1.91217300 & 5.54395500 & 1.98743300 \\
\hline $\mathrm{H}$ & -4.36382400 & 3.73600400 & 1.66908800 \\
\hline $\mathrm{H}$ & -2.15345400 & 2.53180300 & 2.55601900 \\
\hline $\mathrm{H}$ & -3.02794300 & 2.14985000 & 0.27527200 \\
\hline $\mathrm{H}$ & -0.62780800 & 4.03079100 & 0.38623100 \\
\hline $\mathrm{H}$ & -4.16095900 & 4.15831100 & -0.75060800 \\
\hline $\mathrm{H}$ & -2.84329800 & 4.82257400 & 3.29345000 \\
\hline $\mathrm{H}$ & -4.30751600 & 5.46449300 & 1.34583700 \\
\hline $\mathrm{H}$ & -0.71889500 & 3.53694000 & 2.78260600 \\
\hline $\mathrm{C}$ & -0.54196100 & -2.13399600 & 0.66960300 \\
\hline $\mathrm{O}$ & 0.22165600 & -1.28347600 & 1.11247600 \\
\hline $\mathrm{O}$ & -0.11837600 & -3.29227600 & 0.14177500 \\
\hline $\mathrm{C}$ & 1.29985100 & -3.52065700 & 0.10804700 \\
\hline $\mathrm{H}$ & 1.77495300 & -2.72311700 & -0.47166600 \\
\hline $\mathrm{H}$ & 1.69165600 & -3.47312400 & 1.12876100 \\
\hline $\mathrm{C}$ & 1.51742200 & -4.88012800 & -0.51313500 \\
\hline $\mathrm{H}$ & 1.12451800 & -4.91177300 & -1.53318000 \\
\hline $\mathrm{H}$ & 1.01750200 & -5.65871200 & 0.06944700 \\
\hline $\mathrm{H}$ & 2.58770100 & -5.09776700 & -0.54379400 \\
\hline $\mathrm{C}$ & -1.99358500 & -2.01211200 & 0.57591100 \\
\hline $\mathrm{N}$ & -2.72950300 & -3.18645700 & 0.40922300 \\
\hline $\mathrm{C}$ & -2.63974900 & -0.95879400 & 1.35054600 \\
\hline $\mathrm{O}$ & -3.87836300 & -1.30030600 & 1.74050000 \\
\hline $\mathrm{C}$ & -4.63556900 & -0.28569400 & 2.40153500 \\
\hline $\mathrm{H}$ & -4.15097800 & -0.03736000 & 3.35252800 \\
\hline
\end{tabular}




\begin{tabular}{|c|c|c|c|}
\hline $\mathrm{H}$ & -4.63473900 & 0.62709300 & 1.79334300 \\
\hline $\mathrm{C}$ & -6.03242500 & -0.83251200 & 2.59029600 \\
\hline $\mathrm{H}$ & -6.01424300 & -1.74274100 & 3.19560900 \\
\hline $\mathrm{H}$ & -6.48020400 & -1.07642800 & 1.62288200 \\
\hline $\mathrm{H}$ & -6.66459700 & -0.09510600 & 3.09442100 \\
\hline $\mathrm{O}$ & -2.17415700 & 0.16013600 & 1.54933500 \\
\hline $\mathrm{C}$ & -2.63058000 & -4.22891400 & -0.18921500 \\
\hline $\mathrm{O}$ & -2.66569700 & -5.28484800 & -0.71256400 \\
\hline $\mathrm{N}$ & -2.07863300 & 2.48807900 & -1.53008900 \\
\hline $\mathrm{H}$ & -1.78536300 & 1.42584600 & -1.38549500 \\
\hline $\mathrm{C}$ & -0.96445400 & 3.21254100 & -2.18519400 \\
\hline $\mathrm{H}$ & -0.76896200 & 2.72962300 & -3.14387200 \\
\hline $\mathrm{H}$ & -0.06174300 & 3.18044500 & -1.57812900 \\
\hline $\mathrm{H}$ & -1.25134200 & 4.25287900 & -2.35457200 \\
\hline $\mathrm{C}$ & -3.23526100 & 2.45400200 & -2.45432500 \\
\hline $\mathrm{H}$ & -2.98372100 & 1.79589300 & -3.28755700 \\
\hline $\mathrm{H}$ & -3.44759800 & 3.45642100 & -2.82986100 \\
\hline $\mathrm{H}$ & -4.11166000 & 2.05102200 & -1.95175100 \\
\hline $\mathrm{C}$ & 6.32586700 & 1.68705600 & -1.12493900 \\
\hline $\mathrm{C}$ & 4.87813600 & -2.61786800 & 0.99305700 \\
\hline $\mathrm{F}$ & 5.82541900 & 2.43408800 & -2.12683600 \\
\hline $\mathrm{F}$ & 7.33521600 & 0.95271900 & -1.63311900 \\
\hline $\mathrm{F}$ & 6.86655100 & 2.53751900 & -0.23128100 \\
\hline $\mathrm{F}$ & 6.17565600 & -2.83741700 & 1.25946100 \\
\hline $\mathrm{F}$ & 4.52814300 & -3.47833500 & 0.00457300 \\
\hline $\mathrm{F}$ & 4.16774800 & -2.97947200 & 2.08033400 \\
\hline $\mathrm{C}$ & -1.96644500 & -1.05989700 & -1.33006500 \\
\hline $\mathrm{O}$ & -1.36650600 & 0.05492100 & -1.23234800 \\
\hline $\mathrm{H}$ & -1.38640600 & -1.91662600 & -1.71433600 \\
\hline $\mathrm{C}$ & -3.42490700 & -1.17641600 & -1.62241200 \\
\hline $\mathrm{C}$ & -6.16189100 & -1.46983000 & -2.13589500 \\
\hline $\mathrm{C}$ & -3.88598600 & -2.22246000 & -2.42435300 \\
\hline $\mathrm{C}$ & -4.35175500 & -0.29019800 & -1.06719600 \\
\hline $\mathrm{C}$ & -5.71031300 & -0.43115800 & -1.31900400 \\
\hline $\mathrm{C}$ & -5.24749200 & -2.36586000 & -2.68494100 \\
\hline $\mathrm{H}$ & -3.17416900 & -2.92949400 & -2.84452200 \\
\hline $\mathrm{H}$ & -3.99430200 & 0.48453000 & -0.39720000 \\
\hline $\mathrm{H}$ & -6.42174000 & 0.25725100 & -0.86890500 \\
\hline $\mathrm{H}$ & -5.59418800 & -3.18330600 & -3.31115400 \\
\hline $\mathrm{H}$ & -7.22418700 & -1.58542600 & -2.33332900 \\
\hline
\end{tabular}




\section{TS-C ( $\omega B$ 97XD)}

Zero-point correction $=$

Thermal correction to Energy=

Thermal correction to Enthalpy=

Thermal correction to Gibbs Free Energy=

Sum of electronic and zero-point Energies=

Sum of electronic and thermal Energies=

Sum of electronic and thermal Enthalpies=

Sum of electronic and thermal Free Energies=
0.695803 (Hartree/Particle)

0.744080

0.745024

0.607911

$-2908.557198$

$-2908.508921$

$-2908.507977$

$-2908.645089$

$\mathrm{E}(\omega \mathrm{B} 97 \mathrm{XD})=-2909.25300076$

Imaginary frequency $=195 \mathrm{i}$

Relative energy $(\omega \mathrm{B} 97 \mathrm{XD} / 6-311+\mathrm{G}(\mathrm{d}, \mathrm{p}))=-2909.92003794$

01

$\mathrm{C}$

C

C

$\mathrm{C}$

$\mathrm{C}$

$\mathrm{C}$

$\mathrm{H}$

$\mathrm{H}$

$\mathrm{H}$

$\mathrm{N}$

$\mathrm{H}$

$\mathrm{C}$

S

N

$\mathrm{H}$

C

$\mathrm{C}$

C

$\mathrm{C}$

$\mathrm{C}$

C

$\mathrm{H}$

$\mathrm{H}$

$\begin{array}{rrr}5.32747800 & 0.78599400 & -0.49658200 \\ 3.35555000 & -0.71237600 & 0.71692900 \\ 4.05090100 & 1.32563400 & -0.36375100 \\ 5.63295400 & -0.49467900 & -0.05526100 \\ 4.62591000 & -1.23898000 & 0.54917600 \\ 3.04921100 & 0.57152300 & 0.25073100 \\ 3.83362700 & 2.31838300 & -0.73053700 \\ 6.62704200 & -0.90379700 & -0.18206800 \\ 2.57862900 & -1.29121900 & 1.20324900 \\ 1.70978800 & 0.95291300 & 0.39794500 \\ 1.09111100 & 0.16077800 & 0.58252200 \\ 1.10009800 & 2.16563400 & 0.39206100 \\ 1.87246600 & 3.65245500 & 0.16234500 \\ -0.23946100 & 2.05706300 & 0.58044100 \\ -0.65037400 & 1.14928600 & 0.81861500 \\ -1.12159200 & 3.19343800 & 0.70362500 \\ -3.25193400 & 4.28297400 & -0.05332900 \\ -2.35369500 & 4.73607900 & 2.27417300 \\ -3.60383200 & 4.61255800 & 1.40317400 \\ -1.47946000 & 3.48731900 & 2.16597900 \\ -2.41249500 & 3.00671200 & -0.11035700 \\ -2.69163700 & 5.11723900 & -0.49479700 \\ -1.77355100 & 5.61123100 & 1.95402800\end{array}$




\begin{tabular}{|c|c|c|c|}
\hline $\mathrm{H}$ & -4.24698900 & 3.81518800 & 1.79891600 \\
\hline $\mathrm{H}$ & -1.98927800 & 2.61208700 & 2.58647300 \\
\hline $\mathrm{H}$ & -2.97405900 & 2.17793500 & 0.33646700 \\
\hline $\mathrm{H}$ & -0.57483200 & 4.05746400 & 0.31270900 \\
\hline $\mathrm{H}$ & -4.17620100 & 4.16975600 & -0.62699600 \\
\hline $\mathrm{H}$ & -2.63629400 & 4.91027300 & 3.31668100 \\
\hline $\mathrm{H}$ & -4.19011500 & 5.53585500 & 1.43384800 \\
\hline $\mathrm{H}$ & -0.54292100 & 3.62057100 & 2.71431500 \\
\hline $\mathrm{C}$ & -0.58004400 & -2.13583500 & 0.67401700 \\
\hline $\mathrm{O}$ & 0.21071300 & -1.28987600 & 1.07024900 \\
\hline $\mathrm{O}$ & -0.17987200 & -3.31858600 & 0.18816100 \\
\hline $\mathrm{C}$ & 1.23260500 & -3.56533800 & 0.12246000 \\
\hline $\mathrm{H}$ & 1.70031100 & -2.79787500 & -0.50166100 \\
\hline $\mathrm{H}$ & 1.65822400 & -3.48872800 & 1.12695100 \\
\hline $\mathrm{C}$ & 1.41943600 & -4.94956200 & -0.45642800 \\
\hline $\mathrm{H}$ & 0.98150200 & -5.01555100 & -1.45529900 \\
\hline $\mathrm{H}$ & 0.94429100 & -5.70163700 & 0.17767000 \\
\hline $\mathrm{H}$ & 2.48686600 & -5.16883200 & -0.52474200 \\
\hline $\mathrm{C}$ & -2.03050600 & -1.98476100 & 0.59033700 \\
\hline $\mathrm{N}$ & -2.78664600 & -3.16283600 & 0.47346800 \\
\hline $\mathrm{C}$ & -2.65380700 & -0.91347200 & 1.36024100 \\
\hline $\mathrm{O}$ & -3.89192200 & -1.22421700 & 1.76985800 \\
\hline $\mathrm{C}$ & -4.63431300 & -0.18581400 & 2.40773300 \\
\hline $\mathrm{H}$ & -4.15667400 & 0.06860600 & 3.35925600 \\
\hline $\mathrm{H}$ & -4.61659300 & 0.71546500 & 1.78330900 \\
\hline $\mathrm{C}$ & -6.04463900 & -0.70198600 & 2.59481500 \\
\hline $\mathrm{H}$ & -6.04648900 & -1.60599200 & 3.20808400 \\
\hline $\mathrm{H}$ & -6.49104000 & -0.94317900 & 1.62675300 \\
\hline $\mathrm{H}$ & -6.65935600 & 0.05545600 & 3.08896200 \\
\hline $\mathrm{O}$ & -2.17366000 & 0.19998300 & 1.54704800 \\
\hline $\mathrm{C}$ & -2.70502600 & -4.23658900 & -0.06338800 \\
\hline $\mathrm{O}$ & -2.75644100 & -5.31717200 & -0.52934800 \\
\hline $\mathrm{N}$ & -2.12224800 & 2.53131300 & -1.51654700 \\
\hline $\mathrm{H}$ & -1.84291700 & 1.49041900 & -1.40331900 \\
\hline $\mathrm{C}$ & -1.02255400 & 3.24235300 & -2.21795700 \\
\hline $\mathrm{H}$ & -0.89713700 & 2.77743000 & -3.19628100 \\
\hline $\mathrm{H}$ & -0.09030500 & 3.16534400 & -1.66309500 \\
\hline $\mathrm{H}$ & -1.28803200 & 4.29394600 & -2.34358100 \\
\hline C & -3.32111900 & 2.51021600 & -2.39152600 \\
\hline $\mathrm{H}$ & -3.12054300 & 1.82805700 & -3.21842900 \\
\hline
\end{tabular}




$\begin{array}{lrrr}\mathrm{H} & -3.52263900 & 3.51141300 & -2.77436900 \\ \mathrm{H} & -4.18275200 & 2.14019400 & -1.84137800 \\ \mathrm{C} & 6.41165100 & 1.64094000 & -1.09925700 \\ \mathrm{C} & 4.87782200 & -2.66318400 & 0.95735000 \\ \mathrm{~F} & 5.93622800 & 2.44739800 & -2.06138300 \\ \mathrm{~F} & 7.38880300 & 0.89559900 & -1.64516900 \\ \mathrm{~F} & 6.98722400 & 2.42731400 & -0.17403900 \\ \mathrm{~F} & 6.17162400 & -2.89992400 & 1.21264800 \\ \mathrm{~F} & 4.50710800 & -3.51986000 & -0.02048600 \\ \mathrm{~F} & 4.17634300 & -3.00757400 & 2.05160000 \\ \mathrm{C} & -2.04091800 & -1.08879900 & -1.35513500 \\ \mathrm{O} & -1.45270600 & 0.02786400 & -1.31264300 \\ \mathrm{H} & -1.46982600 & -1.95937900 & -1.71978300 \\ \mathrm{C} & -3.50856500 & -1.20962200 & -1.62968200 \\ \mathrm{C} & -6.24268100 & -1.47258300 & -2.16266500 \\ \mathrm{C} & -3.97693900 & -2.25495700 & -2.42425300 \\ \mathrm{C} & -4.42737700 & -0.30597500 & -1.09471500 \\ \mathrm{C} & -5.78455800 & -0.43214700 & -1.35537800 \\ \mathrm{C} & -5.33673000 & -2.38437100 & -2.69379700 \\ \mathrm{H} & -3.27245900 & -2.97541400 & -2.83123800 \\ \mathrm{H} & -4.06762100 & 0.47729400 & -0.43610400 \\ \mathrm{H} & -6.48918900 & 0.27138300 & -0.92125800 \\ \mathrm{H} & -5.68766100 & -3.20249800 & -3.31409200 \\ \mathrm{H} & -7.30345100 & -1.57803100 & -2.36562800\end{array}$

6. Computational study of aldol reaction using aldehyde 27 with isocyanate 13, based on B3LYP/6-31G (d) (in vacuum) using Gaussian 09

The calculation of transition states F-G were first performed using DFT methods at the B3LYP/6-31G(d) level. Once the stationary points were obtained, the harmonic vibrational frequencies were calculated at the same level to estimate the Gibbs free energy. All of the Gibbs free energy values reported in this paper were calculated for a temperature of 298.15 K. In addition, intrinsic reaction coordinate (IRC) calculations were performed at the same level to connect all the transition states with the corresponding reactants and products. Single point energy (SPE) calculations was also performed at the $6-311+\mathrm{G}(\mathrm{d}, \mathrm{p})$ level with several different functionals, such as M062X functional, as well as $\omega \mathrm{B} 97 \mathrm{xD}$ and APFD functionals including dispersion. A self-consistent reaction field (SCRF) method based on the polarizable continuum model (PCM) was used to simulate the solvent effect for toluene. 
(A)

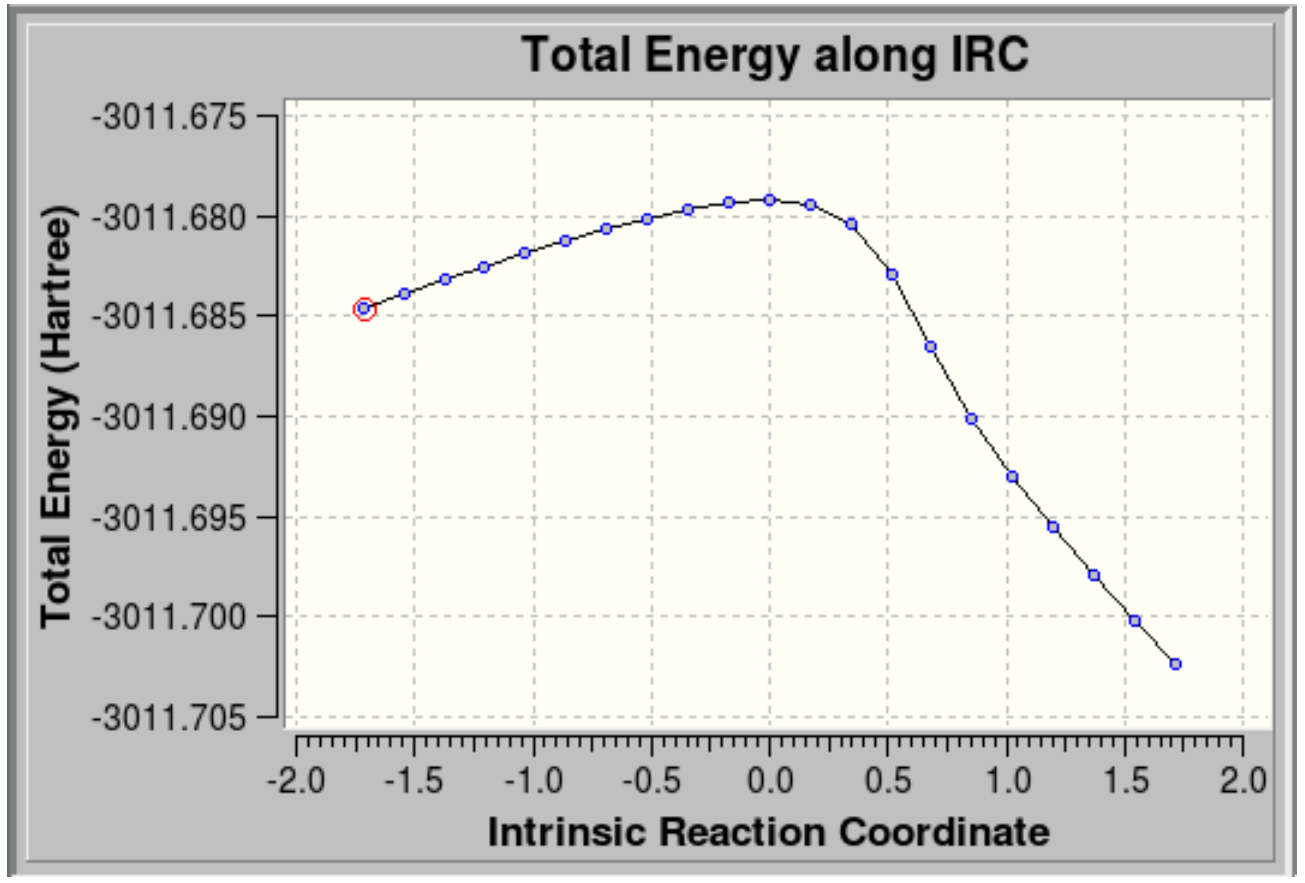

(B)
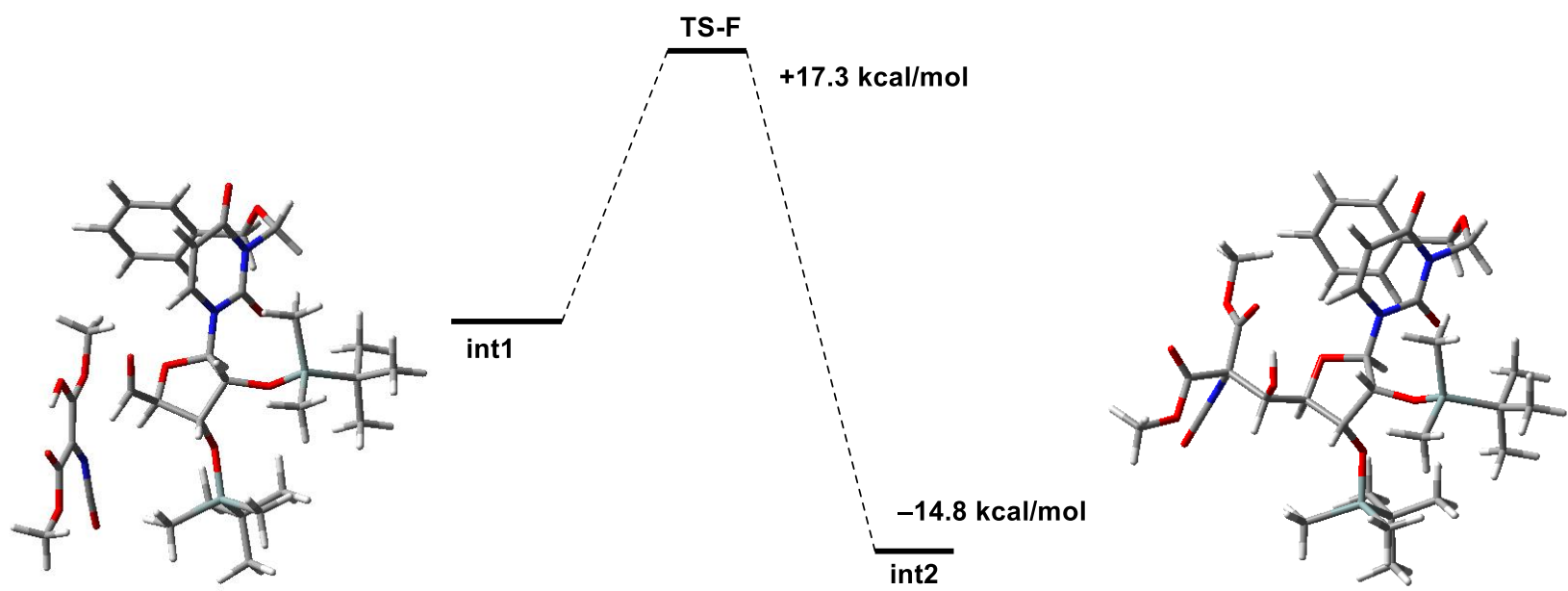

Figure S5. (A) IRC calculations for TS-F (B3LYP/6-31G(d)) (B) Energy diagram for TS-F $(\omega \mathrm{B} 97 \mathrm{xD} / 6-311+\mathrm{G}(\mathrm{d}, \mathrm{p}) / / \mathrm{B} 3 \mathrm{LYP} / 6-31 \mathrm{G}(\mathrm{d}))$

\begin{tabular}{|l|l|}
\hline & $\Delta \mathbf{G}^{\ddagger}\left(\Delta \mathbf{G}_{\text {TS-G }}-\Delta \mathbf{G}_{\text {TS-F }}\right)$ \\
\hline B3LYP/6-311+G(d,p)//B3LYP/6-31G(d) & $+2.10 \mathrm{kcal} / \mathrm{mol}$ \\
\hline M062X/6-311+G(d,p)//B3LYP/6-31G(d) & $+11.6 \mathrm{kcal} / \mathrm{mol}$ \\
\hline APFD/6-311+G(d,p)//B3LYP/6-31G(d) & $+10.8 \mathrm{kcal} / \mathrm{mol}$ \\
\hline $\boldsymbol{\omega B 9 7 X D / 6 - 3 1 1 + G ( d , p ) / / B 3 L Y P / 6 - 3 1 G ( d ) ~}$ & $+10.5 \mathrm{kcal} / \mathrm{mol}$ \\
\hline
\end{tabular}

In each case, the TS-F was suggested to be the favored transition state.

Figure S6. Comparison of the Gibbs free energies of the transition states 
TS-F

Zero-point correction $=$

0.858054 (Hartree/Particle)

Thermal correction to Energy=

0.917865

Thermal correction to Enthalpy=

0.918809

Thermal correction to Gibbs Free Energy=

0.755113

Sum of electronic and zero-point Energies=

$-3010.821189$

Sum of electronic and thermal Energies=

$-3010.761378$

Sum of electronic and thermal Enthalpies=

$-3010.760434$

Sum of electronic and thermal Free Energies=

$-3010.924131$

$E(R B 3 L Y P)=-3011.67924355$

Imaginary frequency $=543 \mathrm{i}$

Relative energy (B3LYP /6-311+G(d,p)) = -3012.42598870

Relative energy (M062X /6-311+G(d,p)) = -3011.39955886

Relative energy (APFD /6-311+G(d,p)) = -3010.34360975

Relative energy $(\omega \mathrm{B} 97 \mathrm{XD} / 6-311+\mathrm{G}(\mathrm{d}, \mathrm{p}))=-3011.65544134$

01

$\mathrm{C}$

$\mathrm{H}$

$\mathrm{C}$

$\mathrm{H}$

$\mathrm{C}$

$\mathrm{H}$

$\mathrm{C}$

$\mathrm{H}$

$\mathrm{O}$

$\mathrm{C}$

$\mathrm{H}$

$\mathrm{O}$

$\mathrm{O}$

$\mathrm{O}$

$\mathrm{N}$

C

$\mathrm{H}$

C

$\mathrm{N}$

C

C

$\begin{array}{rrr}-1.38203200 & -1.21259600 & -0.22354700 \\ -1.79430300 & -1.73898800 & 0.64096700 \\ -2.13051600 & 0.13927100 & -0.38911100 \\ -2.94121200 & 0.07328400 & -1.12720400 \\ -0.98605200 & 1.04573800 & -0.88963200 \\ -0.80309000 & 0.78916800 & -1.94236900 \\ 0.18954100 & 0.52554200 & -0.04314600 \\ 0.17474400 & 0.99504300 & 0.94050100 \\ -0.00809300 & -0.88494200 & 0.04523500 \\ -1.48788400 & -2.08844100 & -1.46909900 \\ -2.51832600 & -2.44939100 & -1.63598500 \\ -0.75684100 & -1.84651200 & -2.49507900 \\ -2.59190500 & 0.58542300 & 0.86199800 \\ -1.18657000 & 2.41400400 & -0.70189600 \\ 1.51234000 & 0.78679300 & -0.60460900 \\ 1.87847800 & 0.19424200 & -1.79679800 \\ 1.12047200 & -0.44822000 & -2.23008900 \\ 2.39308300 & 1.60043900 & 0.12134400 \\ 3.63838000 & 1.80526500 & -0.47246900 \\ 4.07216800 & 1.23690300 & -1.70758900 \\ 3.09052100 & 0.38583700 & -2.35421200\end{array}$




\begin{tabular}{|c|c|c|c|}
\hline $\mathrm{H}$ & 3.36853600 & -0.08628900 & -3.28706900 \\
\hline $\mathrm{O}$ & 5.18695400 & 1.48310100 & -2.14185600 \\
\hline $\mathrm{O}$ & 2.07938100 & 2.09918800 & 1.19474900 \\
\hline $\mathrm{C}$ & 4.59047300 & 2.69963700 & 0.21656400 \\
\hline $\mathrm{H}$ & 3.99534600 & 3.35430600 & 0.85600100 \\
\hline $\mathrm{H}$ & 5.10802000 & 3.26113300 & -0.55882100 \\
\hline $\mathrm{O}$ & 5.59330500 & 2.03390900 & 0.93081900 \\
\hline $\mathrm{C}$ & 5.20047300 & 1.38376600 & 2.13913900 \\
\hline $\mathrm{H}$ & 4.15860700 & 1.62102700 & 2.38503900 \\
\hline $\mathrm{H}$ & 5.82878800 & 1.79414600 & 2.94214700 \\
\hline $\mathrm{C}$ & 5.39858000 & -0.12077600 & 2.07005400 \\
\hline $\mathrm{C}$ & 5.81440800 & -2.89989500 & 2.03236500 \\
\hline $\mathrm{C}$ & 5.95678400 & -0.73665600 & 0.94553000 \\
\hline $\mathrm{C}$ & 5.04679000 & -0.90982900 & 3.17390500 \\
\hline $\mathrm{C}$ & 5.25330400 & -2.28869200 & 3.15827600 \\
\hline $\mathrm{C}$ & 6.16047400 & -2.11971400 & 0.92776500 \\
\hline $\mathrm{H}$ & 6.23440500 & -0.12596500 & 0.09286600 \\
\hline $\mathrm{H}$ & 4.61293500 & -0.44076400 & 4.05530500 \\
\hline $\mathrm{H}$ & 4.98302700 & -2.88531000 & 4.02607700 \\
\hline $\mathrm{H}$ & 6.60367900 & -2.58432200 & 0.05034500 \\
\hline $\mathrm{H}$ & 5.98845500 & -3.97301500 & 2.02262000 \\
\hline $\mathrm{Si}$ & -1.31764800 & 3.62266600 & -1.87655400 \\
\hline $\mathrm{Si}$ & -4.18685300 & 0.60919600 & 1.42057400 \\
\hline $\mathrm{C}$ & -4.98571700 & -1.08479700 & 1.14320300 \\
\hline $\mathrm{H}$ & -4.45445900 & -1.89625300 & 1.65334400 \\
\hline $\mathrm{H}$ & -5.02568400 & -1.33458200 & 0.07504400 \\
\hline $\mathrm{H}$ & -6.02023800 & -1.09002400 & 1.50774100 \\
\hline $\mathrm{C}$ & 0.02213400 & 3.39877600 & -3.19210200 \\
\hline $\mathrm{H}$ & -0.08552800 & 2.44845500 & -3.72893400 \\
\hline $\mathrm{H}$ & -0.04063600 & 4.19591500 & -3.94310300 \\
\hline $\mathrm{H}$ & 1.03072100 & 3.42498500 & -2.76548100 \\
\hline $\mathrm{C}$ & -1.09879300 & 5.24297400 & -0.89281800 \\
\hline $\mathrm{C}$ & -5.17420900 & 1.90122600 & 0.45873100 \\
\hline $\mathrm{H}$ & -5.20000800 & 1.66388800 & -0.61199200 \\
\hline $\mathrm{H}$ & -4.73566900 & 2.89921000 & 0.56582500 \\
\hline $\mathrm{H}$ & -6.21399300 & 1.94843200 & 0.80444500 \\
\hline $\mathrm{C}$ & -3.00970900 & 3.50673800 & -2.71176700 \\
\hline $\mathrm{H}$ & -3.82705300 & 3.60229300 & -1.98869500 \\
\hline $\mathrm{H}$ & -3.12909400 & 2.54321700 & -3.22333800 \\
\hline $\mathrm{H}$ & -3.13647300 & 4.29069900 & -3.46822100 \\
\hline
\end{tabular}




\begin{tabular}{|c|c|c|c|}
\hline $\mathrm{C}$ & -4.02133900 & 1.04742500 & 3.27033700 \\
\hline $\mathrm{C}$ & -1.26961300 & 6.45660500 & -1.83249800 \\
\hline $\mathrm{H}$ & -0.52808400 & 6.46066000 & -2.64127600 \\
\hline $\mathrm{H}$ & -1.14042600 & 7.39257500 & -1.27056700 \\
\hline $\mathrm{H}$ & -2.26644100 & 6.48846600 & -2.28970600 \\
\hline $\mathrm{C}$ & -2.15435000 & 5.32156300 & 0.23210200 \\
\hline $\mathrm{H}$ & -2.02133800 & 6.24595200 & 0.81259100 \\
\hline $\mathrm{H}$ & -3.17724400 & 5.33314800 & -0.16556600 \\
\hline $\mathrm{H}$ & -2.07002500 & 4.47693300 & 0.92428500 \\
\hline $\mathrm{C}$ & 0.31032400 & 5.28449900 & -0.26054600 \\
\hline $\mathrm{H}$ & 1.09843700 & 5.29597200 & -1.02394700 \\
\hline $\mathrm{H}$ & 0.49179400 & 4.42851900 & 0.39882100 \\
\hline $\mathrm{H}$ & 0.42848000 & 6.19873400 & 0.33900300 \\
\hline $\mathrm{C}$ & -3.32669000 & 2.41838400 & 3.42200200 \\
\hline $\mathrm{H}$ & -3.20107600 & 2.66429200 & 4.48637800 \\
\hline $\mathrm{H}$ & -3.91261600 & 3.22728000 & 2.96863400 \\
\hline $\mathrm{H}$ & -2.33428800 & 2.42173400 & 2.95819200 \\
\hline $\mathrm{C}$ & -3.17403100 & -0.02595700 & 3.98811100 \\
\hline $\mathrm{H}$ & -3.63939500 & -1.01820700 & 3.93948200 \\
\hline $\mathrm{H}$ & -3.06133400 & 0.22826300 & 5.05185700 \\
\hline $\mathrm{H}$ & -2.16986600 & -0.10232100 & 3.55680700 \\
\hline $\mathrm{C}$ & -5.41961100 & 1.10921300 & 3.92357000 \\
\hline $\mathrm{H}$ & -5.32940300 & 1.36346400 & 4.98927300 \\
\hline $\mathrm{H}$ & -5.94619700 & 0.14857200 & 3.86385700 \\
\hline $\mathrm{H}$ & -6.05867500 & 1.87243700 & 3.46216700 \\
\hline $\mathrm{C}$ & 0.52235200 & -3.69859000 & -0.76359100 \\
\hline $\mathrm{O}$ & 1.19866100 & -3.83259700 & 0.34632300 \\
\hline $\mathrm{C}$ & 2.54757000 & -3.29657300 & 0.39194000 \\
\hline $\mathrm{H}$ & 2.90145300 & -3.50927600 & 1.39792400 \\
\hline $\mathrm{H}$ & 2.51519500 & -2.22158800 & 0.21364100 \\
\hline $\mathrm{H}$ & 3.17336000 & -3.78880200 & -0.35395700 \\
\hline $\mathrm{C}$ & -0.89487400 & -3.96528500 & -0.75106300 \\
\hline $\mathrm{C}$ & -1.42270900 & -4.74937700 & -1.91480500 \\
\hline $\mathrm{O}$ & -0.88913300 & -4.84097400 & -2.99909800 \\
\hline $\mathrm{N}$ & -1.44387100 & -4.19926900 & 0.51898700 \\
\hline $\mathrm{O}$ & -2.61927300 & -5.30211600 & -1.61214500 \\
\hline $\mathrm{C}$ & -3.23636700 & -6.05033900 & -2.67577800 \\
\hline $\mathrm{H}$ & -4.17895400 & -6.41133600 & -2.26484200 \\
\hline $\mathrm{H}$ & -3.40916900 & -5.40725400 & -3.54209900 \\
\hline $\mathrm{H}$ & -2.59754400 & -6.88597500 & -2.97161600 \\
\hline
\end{tabular}




$\begin{array}{lrrr}\mathrm{O} & 1.09829200 & -3.26314300 & -1.82382800 \\ \mathrm{H} & 0.35934000 & -2.69615600 & -2.37012200 \\ \mathrm{C} & -2.49384700 & -4.59585000 & 0.97535000 \\ \mathrm{O} & -3.45072300 & -4.94362000 & 1.56741300\end{array}$

\section{TS-F-int1 (substrate)}

Zero-point correction $=$ 0.859627 (Hartree/Particle)

Thermal correction to Energy= 0.921019

Thermal correction to Enthalpy= 0.921963

Thermal correction to Gibbs Free Energy= 0.754186

Sum of electronic and zero-point Energies= $-3010.848788$

Sum of electronic and thermal Energies= $-3010.787397$

Sum of electronic and thermal Enthalpies= $-3010.786453$

Sum of electronic and thermal Free Energies= $-3010.954229$

$E(R B 3 L Y P)=-3011.70841575$

Relative energy (M062X /6-311+G(d,p)) = -3011.41978198

Relative energy (APFD $/ 6-311+\mathrm{G}(\mathrm{d}, \mathrm{p}))=-3010.36167672$

Relative energy $(\omega \mathrm{B} 97 \mathrm{XD} / 6-311+\mathrm{G}(\mathrm{d}, \mathrm{p}))=-3011.68208443$

$\begin{array}{crrr}01 & & & \\ \mathrm{C} & -1.33148000 & -0.19912500 & -1.51038400 \\ \mathrm{H} & -2.16888300 & -0.73551700 & -1.05299500 \\ \mathrm{C} & -1.39531400 & 1.30060700 & -1.10222700 \\ \mathrm{H} & -1.93046800 & 1.91140000 & -1.84316200 \\ \mathrm{C} & 0.11582000 & 1.63375000 & -1.08909300 \\ \mathrm{H} & 0.44997900 & 1.68213200 & -2.13682900 \\ \mathrm{C} & 0.69463400 & 0.35201600 & -0.45909900 \\ \mathrm{H} & 0.62944300 & 0.40691700 & 0.62758000 \\ \mathrm{O} & -0.10552100 & -0.70751300 & -0.98188400 \\ \mathrm{C} & -1.40449600 & -0.36649100 & -3.02024700 \\ \mathrm{H} & -2.43714500 & -0.39668000 & -3.42797500 \\ \mathrm{O} & -0.44132000 & -0.42795200 & -3.75418800 \\ \mathrm{O} & -1.94032700 & 1.42568100 & 0.18271600 \\ \mathrm{O} & 0.45642200 & 2.77995600 & -0.37229900 \\ \mathrm{~N} & 2.09465900 & 0.08358700 & -0.78170000 \\ \mathrm{C} & 2.44308200 & -0.30178900 & -2.06204300 \\ \mathrm{H} & 1.60949400 & -0.39761100 & -2.74999200 \\ \mathrm{C} & 3.03725500 & 0.16994900 & 0.25162500\end{array}$




\begin{tabular}{|c|c|c|c|}
\hline $\mathrm{N}$ & 4.35533100 & -0.08349800 & -0.12468000 \\
\hline $\mathrm{C}$ & 4.78661600 & -0.45847100 & -1.43304700 \\
\hline $\mathrm{C}$ & 3.71847000 & -0.56524200 & -2.40892200 \\
\hline $\mathrm{H}$ & 3.98174000 & -0.87166800 & -3.41256800 \\
\hline $\mathrm{O}$ & 5.97061100 & -0.65740400 & -1.65768700 \\
\hline $\mathrm{O}$ & 2.71712700 & 0.46375600 & 1.39679200 \\
\hline $\mathrm{C}$ & 5.40461100 & 0.05163700 & 0.90652500 \\
\hline $\mathrm{H}$ & 5.00640500 & 0.72449000 & 1.66862500 \\
\hline $\mathrm{H}$ & 6.27355400 & 0.47936800 & 0.41017300 \\
\hline $\mathrm{O}$ & 5.84206100 & -1.16084000 & 1.45095500 \\
\hline $\mathrm{C}$ & 4.95030300 & -1.82667300 & 2.34517700 \\
\hline $\mathrm{H}$ & 4.10543000 & -1.17711800 & 2.60346400 \\
\hline $\mathrm{H}$ & 5.51457300 & -2.01611700 & 3.26890500 \\
\hline $\mathrm{C}$ & 4.44989200 & -3.14750300 & 1.78619700 \\
\hline $\mathrm{C}$ & 3.54811500 & -5.63530500 & 0.84147300 \\
\hline $\mathrm{C}$ & 4.90823400 & -3.64798100 & 0.56378000 \\
\hline $\mathrm{C}$ & 3.53427600 & -3.90332600 & 2.53088700 \\
\hline $\mathrm{C}$ & 3.08761300 & -5.14001500 & 2.06571600 \\
\hline $\mathrm{C}$ & 4.45494400 & -4.88357500 & 0.09357700 \\
\hline $\mathrm{H}$ & 5.62137600 & -3.06608600 & -0.01062500 \\
\hline $\mathrm{H}$ & 3.17189400 & -3.52457400 & 3.48500700 \\
\hline $\mathrm{H}$ & 2.38572200 & -5.71947000 & 2.66063600 \\
\hline $\mathrm{H}$ & 4.82079900 & -5.26159200 & -0.85781800 \\
\hline $\mathrm{H}$ & 3.20736900 & -6.60230500 & 0.48007300 \\
\hline $\mathrm{Si}$ & 1.25327000 & 4.15568600 & -0.96076300 \\
\hline $\mathrm{Si}$ & -3.41359000 & 2.14867500 & 0.60139200 \\
\hline $\mathrm{C}$ & -4.81883500 & 1.24736800 & -0.29275600 \\
\hline $\mathrm{H}$ & -4.88763400 & 0.19460100 & 0.00370600 \\
\hline $\mathrm{H}$ & -4.68332200 & 1.28239200 & -1.38166800 \\
\hline $\mathrm{H}$ & -5.78538000 & 1.71811200 & -0.07589100 \\
\hline $\mathrm{C}$ & 2.74711400 & 3.63781200 & -1.99533700 \\
\hline $\mathrm{H}$ & 2.45233000 & 3.06483500 & -2.88270700 \\
\hline $\mathrm{H}$ & 3.29115600 & 4.52070200 & -2.35220000 \\
\hline $\mathrm{H}$ & 3.45143300 & 3.02308800 & -1.42493400 \\
\hline $\mathrm{C}$ & 1.75292200 & 5.11189200 & 0.61195200 \\
\hline $\mathrm{C}$ & -3.39874100 & 3.95478300 & 0.04817900 \\
\hline $\mathrm{H}$ & -3.27166500 & 4.04140300 & -1.03807100 \\
\hline $\mathrm{H}$ & -2.58026300 & 4.50732300 & 0.52163900 \\
\hline $\mathrm{H}$ & -4.33981100 & 4.45670300 & 0.30318700 \\
\hline $\mathrm{C}$ & 0.06273000 & 5.12867500 & -2.06135800 \\
\hline
\end{tabular}




\begin{tabular}{|c|c|c|c|}
\hline $\mathrm{H}$ & -0.83285200 & 5.44584100 & -1.51564300 \\
\hline $\mathrm{H}$ & -0.26657000 & 4.52012800 & -2.91332100 \\
\hline $\mathrm{H}$ & 0.54041200 & 6.02580000 & -2.47314400 \\
\hline $\mathrm{C}$ & -3.52181400 & 1.95848000 & 2.49451500 \\
\hline $\mathrm{C}$ & 2.36840000 & 6.47547000 & 0.22764200 \\
\hline $\mathrm{H}$ & 3.26489600 & 6.36445800 & -0.39533400 \\
\hline $\mathrm{H}$ & 2.66976500 & 7.02274200 & 1.13201000 \\
\hline $\mathrm{H}$ & 1.65819300 & 7.11162100 & -0.31494000 \\
\hline $\mathrm{C}$ & 0.50923300 & 5.34811300 & 1.49553300 \\
\hline $\mathrm{H}$ & 0.79096700 & 5.88962900 & 2.41016100 \\
\hline $\mathrm{H}$ & -0.24947700 & 5.95266100 & 0.98190900 \\
\hline $\mathrm{H}$ & 0.04481300 & 4.40306000 & 1.79516800 \\
\hline $\mathrm{C}$ & 2.79057900 & 4.29285800 & 1.41243500 \\
\hline $\mathrm{H}$ & 3.72835200 & 4.17141100 & 0.85601000 \\
\hline $\mathrm{H}$ & 2.42125100 & 3.29375700 & 1.66884300 \\
\hline $\mathrm{H}$ & 3.03657300 & 4.81040100 & 2.35086800 \\
\hline $\mathrm{C}$ & -2.32445600 & 2.67020800 & 3.16162000 \\
\hline $\mathrm{H}$ & -2.37149900 & 2.54588100 & 4.25295600 \\
\hline $\mathrm{H}$ & -2.32098100 & 3.74786200 & 2.95689800 \\
\hline $\mathrm{H}$ & -1.36783300 & 2.26002700 & 2.82009200 \\
\hline $\mathrm{C}$ & -3.49512300 & 0.46291100 & 2.87967800 \\
\hline $\mathrm{H}$ & -4.36163200 & -0.08161400 & 2.48575200 \\
\hline $\mathrm{H}$ & -3.51936300 & 0.35482400 & 3.97322600 \\
\hline $\mathrm{H}$ & -2.58819400 & -0.03424400 & 2.51807900 \\
\hline $\mathrm{C}$ & -4.83701600 & 2.58845600 & 3.00520400 \\
\hline $\mathrm{H}$ & -4.90762100 & 2.48102300 & 4.09673400 \\
\hline $\mathrm{H}$ & -5.72176100 & 2.10220700 & 2.57571800 \\
\hline $\mathrm{H}$ & -4.89781000 & 3.66068100 & 2.78046900 \\
\hline $\mathrm{C}$ & -2.15267200 & -3.57318600 & -0.75763000 \\
\hline $\mathrm{O}$ & -1.22982800 & -3.98188600 & 0.08621600 \\
\hline $\mathrm{C}$ & 0.11735500 & -4.16598000 & -0.41144800 \\
\hline $\mathrm{H}$ & 0.69852500 & -4.46838800 & 0.45790900 \\
\hline $\mathrm{H}$ & 0.49168300 & -3.22456800 & -0.81665200 \\
\hline $\mathrm{H}$ & 0.13585100 & -4.94466700 & -1.17725400 \\
\hline $\mathrm{C}$ & -3.42652300 & -3.24629100 & -0.29873300 \\
\hline $\mathrm{C}$ & -4.39611700 & -2.79280400 & -1.26247500 \\
\hline $\mathrm{O}$ & -4.16301900 & -2.65349100 & -2.48249100 \\
\hline $\mathrm{N}$ & -3.71553300 & -3.37658800 & 1.05732200 \\
\hline $\mathrm{O}$ & -5.59775900 & -2.50460800 & -0.73504700 \\
\hline $\mathrm{C}$ & -6.61237000 & -2.07243900 & -1.65878200 \\
\hline
\end{tabular}




$\begin{array}{lrrr}\mathrm{H} & -7.50941100 & -1.93898700 & -1.05456600 \\ \mathrm{H} & -6.32315800 & -1.13028700 & -2.13052400 \\ \mathrm{H} & -6.77360200 & -2.82948700 & -2.42969500 \\ \mathrm{O} & -1.81235600 & -3.48547600 & -2.03218600 \\ \mathrm{H} & -2.64778400 & -3.19218800 & -2.52325500 \\ \mathrm{C} & -4.63628200 & -3.14720400 & 1.80626500 \\ \mathrm{O} & -5.44513200 & -2.97264500 & 2.64837300\end{array}$

\section{TS-F-int2 (product)}

Zero-point correction $=$ 0.863503 (Hartree/Particle)

Thermal correction to Energy=

0.923535

Thermal correction to Enthalpy=

0.924479

Thermal correction to Gibbs Free Energy=

0.760705

Sum of electronic and zero-point Energies= $-3010.861745$

Sum of electronic and thermal Energies=

$-3010.801714$

Sum of electronic and thermal Enthalpies=

$-3010.800769$

Sum of electronic and thermal Free Energies=

$-3010.964544$

$\mathrm{E}(\mathrm{RB} 3 \mathrm{LYP})=-3011.72524845$

Relative energy $(\mathrm{M} 062 \mathrm{X} / 6-311+\mathrm{G}(\mathrm{d}, \mathrm{p}))=-3011.45338561$

Relative energy (APFD $/ 6-311+G(d, p))=-3010.39342250$

Relative energy $(\omega \mathrm{B} 97 \mathrm{XD} / 6-311+\mathrm{G}(\mathrm{d}, \mathrm{p}))=-3011.71216774$

01

$\mathrm{C}$

$\mathrm{H}$

C

$\mathrm{H}$

C

$\mathrm{H}$

$\mathrm{C}$

$\mathrm{H}$

$\mathrm{O}$

$\mathrm{C}$

$\mathrm{H}$

$\mathrm{O}$

$\mathrm{O}$

$\mathrm{O}$

$\mathrm{N}$

$\begin{array}{rrr}1.81512000 & 0.36720400 & 0.01354300 \\ 2.24136000 & 0.45338300 & 1.01837600 \\ 1.63457700 & -1.14075900 & -0.31568200 \\ 2.36975600 & -1.48744300 & -1.05139100 \\ 0.20700600 & -1.15927200 & -0.90667300 \\ 0.27467500 & -0.75795900 & -1.92852500 \\ -0.46942600 & -0.09526500 & -0.02386300 \\ -0.68405000 & -0.51021800 & 0.96234100 \\ 0.49633000 & 0.95270000 & 0.02799600 \\ 2.73795800 & 1.08560000 & -0.98091400 \\ 3.73418200 & 0.65259600 & -0.83223800 \\ 2.40193400 & 0.86399700 & -2.33350500 \\ 1.67628600 & -1.89889000 & 0.87286800 \\ -0.43725300 & -2.39602100 & -0.85731600 \\ -1.72789100 & 0.44302400 & -0.53030800\end{array}$




\begin{tabular}{|c|c|c|c|}
\hline $\mathrm{C}$ & -1.73387900 & 1.31692400 & -1.59627200 \\
\hline $\mathrm{H}$ & -0.75529000 & 1.59237400 & -1.96539700 \\
\hline $\mathrm{C}$ & -2.91048200 & 0.06457500 & 0.12180400 \\
\hline $\mathrm{N}$ & -4.08404600 & 0.57466100 & -0.43130000 \\
\hline $\mathrm{C}$ & -4.15986500 & 1.46603600 & -1.54342500 \\
\hline $\mathrm{C}$ & -2.87244200 & 1.82745600 & -2.10660100 \\
\hline $\mathrm{H}$ & -2.86028800 & 2.51951200 & -2.93798500 \\
\hline $\mathrm{O}$ & -5.24277400 & 1.85478900 & -1.95210600 \\
\hline $\mathrm{O}$ & -2.90340700 & -0.67705700 & 1.09558400 \\
\hline $\mathrm{C}$ & -5.36196900 & 0.17337700 & 0.18725000 \\
\hline $\mathrm{H}$ & -5.18972700 & -0.79618600 & 0.65978400 \\
\hline $\mathrm{H}$ & -6.08674500 & 0.10213700 & -0.62119900 \\
\hline $\mathrm{O}$ & -5.89623600 & 1.10209800 & 1.09005200 \\
\hline $\mathrm{C}$ & -5.21420700 & 1.23969900 & 2.33835500 \\
\hline $\mathrm{H}$ & -4.72607200 & 0.29923500 & 2.61968500 \\
\hline $\mathrm{H}$ & -6.00532500 & 1.44221500 & 3.07171100 \\
\hline $\mathrm{C}$ & -4.21364000 & 2.38104000 & 2.35143800 \\
\hline $\mathrm{C}$ & -2.43748100 & 4.55038600 & 2.50229200 \\
\hline $\mathrm{C}$ & -4.46110400 & 3.55176700 & 1.62322600 \\
\hline $\mathrm{C}$ & -3.06090400 & 2.30272700 & 3.14120900 \\
\hline $\mathrm{C}$ & -2.17843200 & 3.38193900 & 3.22065700 \\
\hline $\mathrm{C}$ & -3.57752500 & 4.62877200 & 1.69733200 \\
\hline $\mathrm{H}$ & -5.34149000 & 3.60332300 & 0.9897560 \\
\hline $\mathrm{H}$ & -2.84962600 & 1.38931900 & 3.69253000 \\
\hline $\mathrm{H}$ & -1.28683100 & 3.30665900 & 3.83768600 \\
\hline $\mathrm{H}$ & -3.78452000 & 5.53406900 & 1.13166400 \\
\hline $\mathrm{H}$ & -1.75606300 & 5.39482000 & 2.5708190 \\
\hline $\mathrm{Si}$ & -1.00814500 & -3.29947900 & -2.16804300 \\
\hline $\mathrm{Si}$ & 2.89593300 & -2.95144400 & 1.37157700 \\
\hline $\mathrm{C}$ & 4.55733200 & -2.04091300 & 1.37990400 \\
\hline $\mathrm{H}$ & 4.53790700 & -1.14947300 & 2.01735300 \\
\hline $\mathrm{H}$ & 4.84399900 & -1.72404600 & 0.36891000 \\
\hline $\mathrm{H}$ & 5.36021000 & -2.69147300 & 1.7478300 \\
\hline $\mathrm{C}$ & -2.01686200 & -2.19671200 & -3.32643400 \\
\hline $\mathrm{H}$ & -1.40420900 & -1.40373200 & -3.77224500 \\
\hline $\mathrm{H}$ & -2.42781000 & -2.78471800 & -4.15625800 \\
\hline $\mathrm{H}$ & -2.85695000 & -1.71610200 & -2.81374500 \\
\hline $\mathrm{C}$ & -2.07708600 & -4.65971900 & -1.36359800 \\
\hline $\mathrm{C}$ & 3.01471200 & -4.40997300 & 0.17630800 \\
\hline $\mathrm{H}$ & 3.26799500 & -4.07450400 & -0.83711600 \\
\hline
\end{tabular}




\begin{tabular}{|c|c|c|c|}
\hline $\mathrm{H}$ & 2.06560800 & -4.95321200 & 0.11733700 \\
\hline $\mathrm{H}$ & 3.79292900 & -5.11835400 & 0.48552100 \\
\hline $\mathrm{C}$ & 0.46226900 & -3.99547800 & -3.13078000 \\
\hline $\mathrm{H}$ & 1.08585700 & -4.64654500 & -2.50858100 \\
\hline $\mathrm{H}$ & 1.10077900 & -3.18458800 & -3.50387900 \\
\hline $\mathrm{H}$ & 0.13553800 & -4.57535300 & -4.00250500 \\
\hline $\mathrm{C}$ & 2.36597700 & -3.48651800 & 3.12627700 \\
\hline $\mathrm{C}$ & -2.61083400 & -5.62188300 & -2.44761700 \\
\hline $\mathrm{H}$ & -3.23523300 & -5.10716800 & -3.18879400 \\
\hline $\mathrm{H}$ & -3.23342200 & -6.40256300 & -1.98750500 \\
\hline $\mathrm{H}$ & -1.80010400 & -6.12929300 & -2.98531200 \\
\hline $\mathrm{C}$ & -1.23508800 & -5.46153600 & -0.34758200 \\
\hline $\mathrm{H}$ & -1.85203100 & -6.23891600 & 0.12603000 \\
\hline $\mathrm{H}$ & -0.38687500 & -5.96817700 & -0.82603100 \\
\hline $\mathrm{H}$ & -0.84193200 & -4.81750100 & 0.44598800 \\
\hline $\mathrm{C}$ & -3.26964300 & -4.01105500 & -0.62579900 \\
\hline $\mathrm{H}$ & -3.94109200 & -3.49052700 & -1.32038800 \\
\hline $\mathrm{H}$ & -2.94315800 & -3.28825000 & 0.13006600 \\
\hline $\mathrm{H}$ & -3.86582700 & -4.78291000 & -0.11779100 \\
\hline $\mathrm{C}$ & 0.99348900 & -4.19163500 & 3.06690000 \\
\hline $\mathrm{H}$ & 0.66852600 & -4.47583100 & 4.07827600 \\
\hline $\mathrm{H}$ & 1.02983500 & -5.10966500 & 2.46763200 \\
\hline $\mathrm{H}$ & 0.22342700 & -3.54117000 & 2.63817800 \\
\hline $\mathrm{C}$ & 2.25128300 & -2.24516200 & 4.03759100 \\
\hline $\mathrm{H}$ & 3.20955600 & -1.72115200 & 4.14158100 \\
\hline $\mathrm{H}$ & 1.93224200 & -2.54186100 & 5.04712000 \\
\hline $\mathrm{H}$ & 1.51515800 & -1.53035900 & 3.65360600 \\
\hline $\mathrm{C}$ & 3.41163300 & -4.45624500 & 3.71881200 \\
\hline $\mathrm{H}$ & 3.10995200 & -4.76886100 & 4.72862200 \\
\hline $\mathrm{H}$ & 4.40264300 & -3.99346400 & 3.80581600 \\
\hline $\mathrm{H}$ & 3.51797900 & -5.36707700 & 3.11652500 \\
\hline $\mathrm{C}$ & 1.61219600 & 3.38595100 & -1.03243600 \\
\hline $\mathrm{O}$ & 1.31998100 & 4.33632700 & -0.16336700 \\
\hline $\mathrm{C}$ & 0.11861900 & 5.09422500 & -0.43520100 \\
\hline $\mathrm{H}$ & 0.11735400 & 5.89535500 & 0.30256000 \\
\hline $\mathrm{H}$ & -0.75791900 & 4.45538500 & -0.30913600 \\
\hline $\mathrm{H}$ & 0.14974200 & 5.49444100 & -1.45039800 \\
\hline $\mathrm{C}$ & 2.91228100 & 2.62410100 & -0.69973100 \\
\hline $\mathrm{C}$ & 3.99614900 & 3.17757900 & -1.66987100 \\
\hline $\mathrm{O}$ & 3.80270500 & 4.01918100 & -2.51251600 \\
\hline
\end{tabular}




$\begin{array}{lrrr}\mathrm{N} & 3.30264600 & 2.84169200 & 0.66843900 \\ \mathrm{O} & 5.18140700 & 2.60433500 & -1.41158000 \\ \mathrm{C} & 6.28214900 & 3.05020500 & -2.23204600 \\ \mathrm{H} & 7.15064200 & 2.49938800 & -1.87291800 \\ \mathrm{H} & 6.08114100 & 2.82223100 & -3.28112800 \\ \mathrm{H} & 6.42756500 & 4.12631900 & -2.11401200 \\ \mathrm{O} & 0.96230700 & 3.13854900 & -2.03571700 \\ \mathrm{H} & 1.72714900 & 1.53527100 & -2.56783000 \\ \mathrm{C} & 4.33317900 & 2.74477600 & 1.29967100 \\ \mathrm{O} & 5.24678600 & 2.69681800 & 2.03879000\end{array}$

\section{TS-G}

Zero-point correction $=$

0.858191 (Hartree/Particle)
0.917460
0.918405
0.758462
-3010.803494
-3010.744224
-3010.743280
-3010.903223

Thermal correction to Energy=

Thermal correction to Enthalpy=

Thermal correction to Gibbs Free Energy=

Sum of electronic and zero-point Energies=

Sum of electronic and thermal Energies=

Sum of electronic and thermal Enthalpies=

Sum of electronic and thermal Free Energies=

$\mathrm{E}(\mathrm{RB} 3 \mathrm{LYP})=-3011.66168495$

Imaginary frequency $=753 \mathrm{i}$

Relative energy (B3LYP /6-311+G(d,p)) $=-3012.42598870$

Relative energy $(\mathrm{M} 062 \mathrm{X} / 6-311+\mathrm{G}(\mathrm{d}, \mathrm{p}))=-3011.38447867$

Relative energy (APFD /6-311+G(d,p)) = -3010.32973502

Relative energy $(\omega \mathrm{B} 97 \mathrm{XD} / 6-311+\mathrm{G}(\mathrm{d}, \mathrm{p}))=-3011.64209286$

$\begin{array}{rrrr}01 & & & \\ \mathrm{C} & -1.27121200 & -0.78562500 & 0.19804600 \\ \mathrm{H} & -1.87628300 & -1.06104100 & 1.06468300 \\ \mathrm{C} & -1.36998000 & 0.72633900 & -0.09202700 \\ \mathrm{H} & -2.16141800 & 0.96617800 & -0.81238000 \\ \mathrm{C} & 0.03019400 & 1.00543000 & -0.68399800 \\ \mathrm{H} & 0.02423500 & 0.61508000 & -1.70743100 \\ \mathrm{C} & 0.91706200 & 0.10540700 & 0.19875200 \\ \mathrm{H} & 1.21465400 & 0.64232100 & 1.09864200 \\ \mathrm{O} & 0.11629200 & -1.03125400 & 0.53218600 \\ \mathrm{O} & -1.52330200 & 1.43831800 & 1.11319400\end{array}$




\begin{tabular}{|c|c|c|c|}
\hline $\mathrm{O}$ & 0.44990400 & 2.33859400 & -0.63939000 \\
\hline $\mathrm{N}$ & 2.15418200 & -0.34554300 & -0.43583300 \\
\hline $\mathrm{C}$ & 2.10530800 & -1.12448500 & -1.57450300 \\
\hline $\mathrm{H}$ & 1.11144600 & -1.32055900 & -1.96046100 \\
\hline $\mathrm{C}$ & 3.36712600 & -0.06502400 & 0.21068300 \\
\hline $\mathrm{N}$ & 4.50674600 & -0.57659500 & -0.40569000 \\
\hline $\mathrm{C}$ & 4.52609900 & -1.34680000 & -1.60578800 \\
\hline $\mathrm{C}$ & 3.21361600 & -1.60830600 & -2.16841000 \\
\hline $\mathrm{H}$ & 3.16050100 & -2.21044600 & -3.06558500 \\
\hline $\mathrm{O}$ & 5.58393200 & -1.72422800 & -2.08411100 \\
\hline $\mathrm{O}$ & 3.41273200 & 0.60052200 & 1.23775500 \\
\hline $\mathrm{C}$ & 5.80721000 & -0.32256100 & 0.24344800 \\
\hline $\mathrm{H}$ & 5.69552400 & 0.59909700 & 0.81875500 \\
\hline $\mathrm{H}$ & 6.53582100 & -0.21070500 & -0.55678500 \\
\hline $\mathrm{O}$ & 6.27742800 & -1.37630400 & 1.03885400 \\
\hline $\mathrm{C}$ & 5.57824400 & -1.60350000 & 2.26722400 \\
\hline $\mathrm{H}$ & 5.18911100 & -0.66067400 & 2.66961100 \\
\hline $\mathrm{H}$ & 6.344333300 & -1.97745000 & 2.95774800 \\
\hline $\mathrm{C}$ & 4.46366200 & -2.62509700 & 2.14012500 \\
\hline $\mathrm{C}$ & 2.44370500 & -4.56851400 & 1.97850700 \\
\hline $\mathrm{C}$ & 4.66471400 & -3.79894100 & 1.40109200 \\
\hline $\mathrm{C}$ & 3.23910900 & -2.43295900 & 2.78849600 \\
\hline $\mathrm{C}$ & 2.23315000 & -3.39917600 & 2.70920500 \\
\hline $\mathrm{C}$ & 3.66151600 & -4.76359600 & 1.32053100 \\
\hline $\mathrm{H}$ & 5.60602300 & -3.94025700 & 0.87762400 \\
\hline $\mathrm{H}$ & 3.06482000 & -1.51413300 & 3.34274200 \\
\hline $\mathrm{H}$ & 1.28344700 & -3.23069300 & 3.20985400 \\
\hline $\mathrm{H}$ & 3.82944300 & -5.66915300 & 0.74282500 \\
\hline $\mathrm{H}$ & 1.66216500 & -5.32156500 & 1.91564900 \\
\hline $\mathrm{Si}$ & 0.73296700 & 3.37579300 & -1.94643800 \\
\hline $\mathrm{Si}$ & -2.88981900 & 2.18345000 & 1.75996800 \\
\hline $\mathrm{C}$ & -3.94666000 & 0.88593300 & 2.64626200 \\
\hline $\mathrm{H}$ & -3.42639800 & 0.46803300 & 3.51542800 \\
\hline $\mathrm{H}$ & -4.18276300 & 0.05073600 & 1.97642000 \\
\hline $\mathrm{H}$ & -4.89508700 & 1.31047900 & 2.99724500 \\
\hline $\mathrm{C}$ & 1.12777100 & 2.36699900 & -3.49703400 \\
\hline $\mathrm{H}$ & 1.96722600 & 1.67850500 & -3.34844200 \\
\hline $\mathrm{H}$ & 1.39473800 & 3.03732000 & -4.32298600 \\
\hline $\mathrm{H}$ & 0.26762200 & 1.77567000 & -3.83344300 \\
\hline $\mathrm{C}$ & 2.20291900 & 4.48036300 & -1.42282000 \\
\hline
\end{tabular}




\begin{tabular}{|c|c|c|c|}
\hline $\mathrm{C}$ & -3.90526500 & 2.97568500 & 0.37649300 \\
\hline $\mathrm{H}$ & -3.30974400 & 3.68677500 & -0.20640100 \\
\hline $\mathrm{H}$ & -4.30792900 & 2.22865300 & -0.31774500 \\
\hline $\mathrm{H}$ & -4.76035800 & 3.52206200 & 0.79301900 \\
\hline $\mathrm{C}$ & -0.83074800 & 4.39112200 & -2.25580700 \\
\hline $\mathrm{H}$ & -1.12310500 & 4.95903100 & -1.36538900 \\
\hline $\mathrm{H}$ & -0.69649000 & 5.10294900 & -3.07913600 \\
\hline $\mathrm{H}$ & -1.67028900 & 3.73766400 & -2.52342600 \\
\hline $\mathrm{C}$ & -2.21167000 & 3.47541400 & 2.99374500 \\
\hline $\mathrm{C}$ & 2.52234500 & 5.48656400 & -2.55097700 \\
\hline $\mathrm{H}$ & 1.67201200 & 6.14247900 & -2.77610300 \\
\hline $\mathrm{H}$ & 2.81631700 & 4.98619300 & -3.48200500 \\
\hline $\mathrm{H}$ & 3.36012700 & 6.13358700 & -2.25415700 \\
\hline $\mathrm{C}$ & 1.85606000 & 5.25931100 & -0.13585100 \\
\hline $\mathrm{H}$ & 1.63603000 & 4.58252100 & 0.69672000 \\
\hline $\mathrm{H}$ & 2.70494300 & 5.89024900 & 0.16513100 \\
\hline $\mathrm{H}$ & 0.99160500 & 5.92060400 & -0.27222700 \\
\hline $\mathrm{C}$ & 3.44887000 & 3.60918600 & -1.15192900 \\
\hline $\mathrm{H}$ & 3.74871100 & 3.03345500 & -2.03662100 \\
\hline $\mathrm{H}$ & 3.28122700 & 2.90820200 & -0.32714900 \\
\hline $\mathrm{H}$ & 4.30217400 & 4.24555400 & -0.87574000 \\
\hline $\mathrm{C}$ & -3.38083100 & 4.15410100 & 3.73949800 \\
\hline $\mathrm{H}$ & -4.05805000 & 4.68085100 & 3.05506400 \\
\hline $\mathrm{H}$ & -3.97651500 & 3.43486900 & 4.31512400 \\
\hline $\mathrm{H}$ & -2.99704100 & 4.89972400 & 4.45041700 \\
\hline $\mathrm{C}$ & -1.40140200 & 4.54388300 & 2.23067700 \\
\hline $\mathrm{H}$ & -0.97484500 & 5.27454200 & 2.93312900 \\
\hline $\mathrm{H}$ & -2.02697400 & 5.10127500 & 1.52188200 \\
\hline $\mathrm{H}$ & -0.57528500 & 4.09346600 & 1.67081800 \\
\hline $\mathrm{C}$ & -1.28730600 & 2.78385400 & 4.01933100 \\
\hline $\mathrm{H}$ & -1.82121300 & 2.03331400 & 4.61549500 \\
\hline $\mathrm{H}$ & -0.44338400 & 2.28642100 & 3.52972600 \\
\hline $\mathrm{H}$ & -0.87756300 & 3.52420200 & 4.72156300 \\
\hline $\mathrm{C}$ & -4.09682700 & -1.05419800 & -1.58975400 \\
\hline $\mathrm{O}$ & -5.04455800 & -0.26102300 & -1.11277000 \\
\hline $\mathrm{C}$ & -6.15875700 & -0.76747900 & -0.33975700 \\
\hline $\mathrm{H}$ & -6.40056400 & -1.78952300 & -0.63626500 \\
\hline $\mathrm{H}$ & -6.98673300 & -0.10066500 & -0.58318900 \\
\hline $\mathrm{H}$ & -5.93232100 & -0.72434500 & 0.72471900 \\
\hline $\mathrm{C}$ & -3.58305200 & -2.23351700 & -0.92765300 \\
\hline
\end{tabular}




$\begin{array}{lrrr}\mathrm{C} & -3.43665100 & -3.44739500 & -1.80221000 \\ \mathrm{O} & -3.35794600 & -3.43419900 & -3.00988100 \\ \mathrm{~N} & -3.97946900 & -2.41510100 & 0.41378900 \\ \mathrm{O} & -3.35828700 & -4.56458500 & -1.04615900 \\ \mathrm{C} & -3.17191800 & -5.79336900 & -1.77631900 \\ \mathrm{H} & -4.01479900 & -5.96375500 & -2.45018700 \\ \mathrm{H} & -3.11827800 & -6.57327800 & -1.01738700 \\ \mathrm{H} & -2.24832200 & -5.74976300 & -2.35785100 \\ \mathrm{O} & -3.55554100 & -0.63252600 & -2.66260200 \\ \mathrm{H} & -2.49130700 & -0.95209000 & -2.66446500 \\ \mathrm{C} & -3.93367700 & -3.33818100 & 1.20568800 \\ \mathrm{O} & -3.95749000 & -4.11762700 & 2.08385200 \\ \mathrm{C} & -1.52993100 & -1.73522400 & -0.98074800 \\ \mathrm{H} & -1.24962600 & -2.76704900 & -0.71731500 \\ \mathrm{O} & -1.31724300 & -1.34787400 & -2.18821900\end{array}$



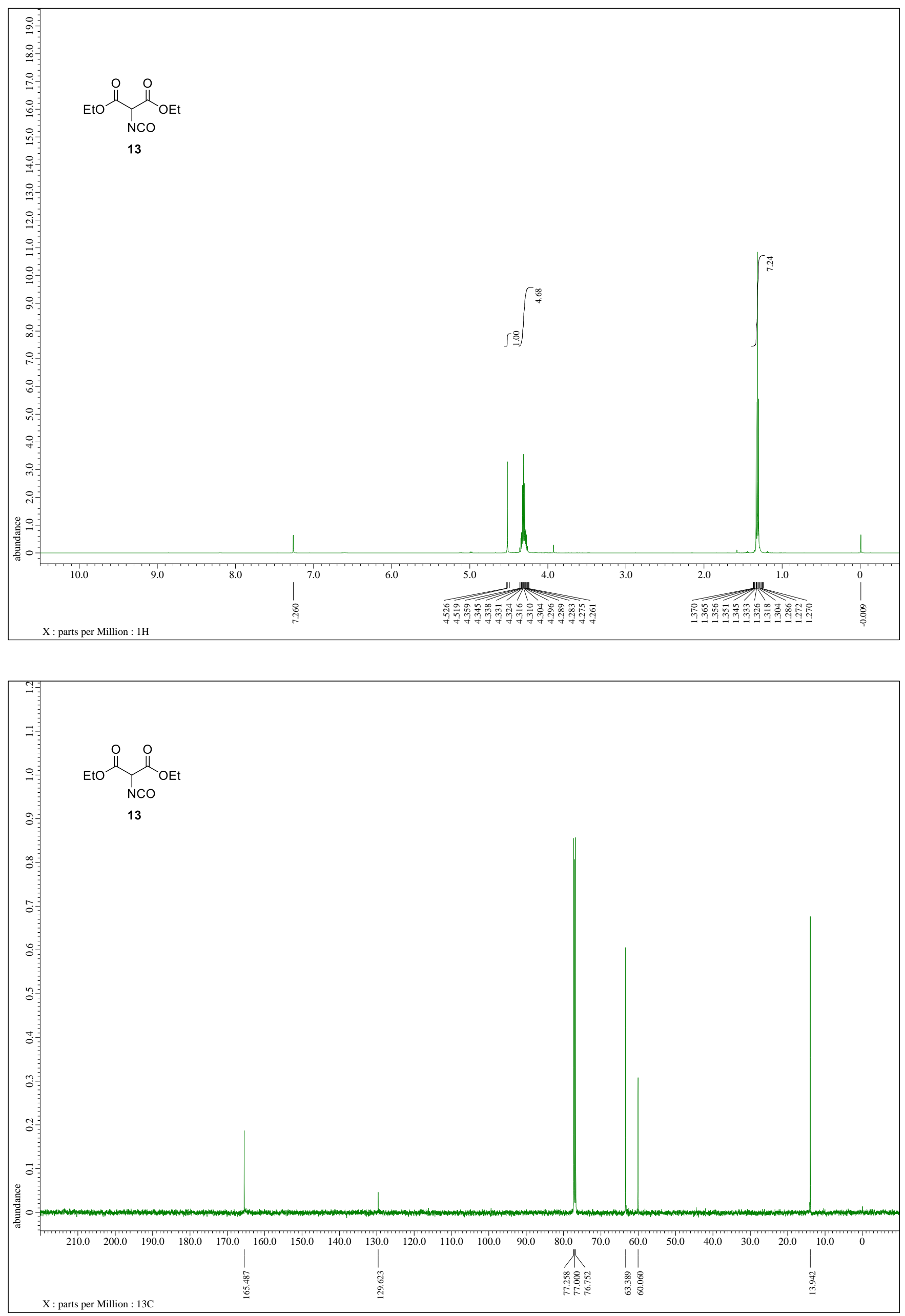

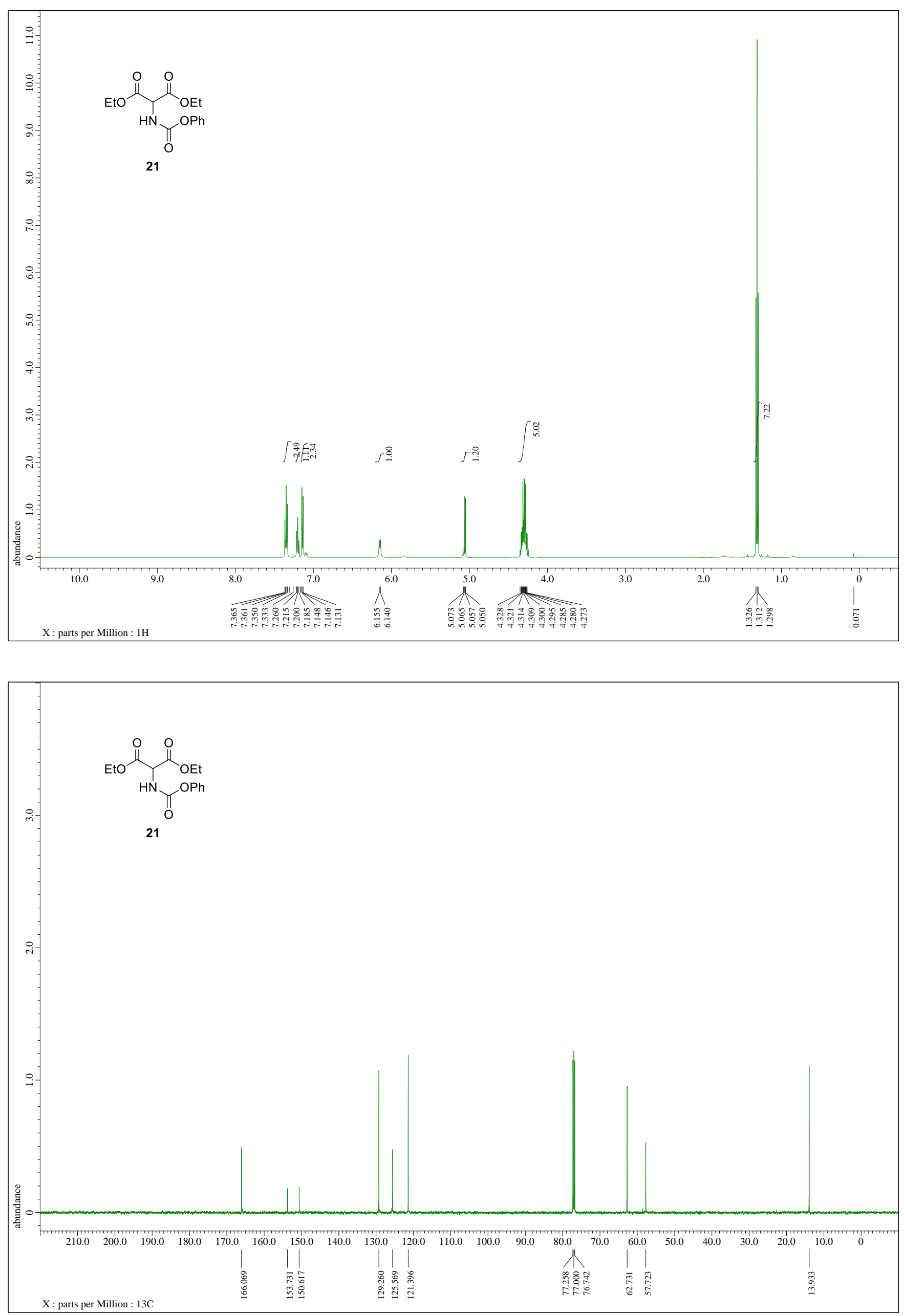

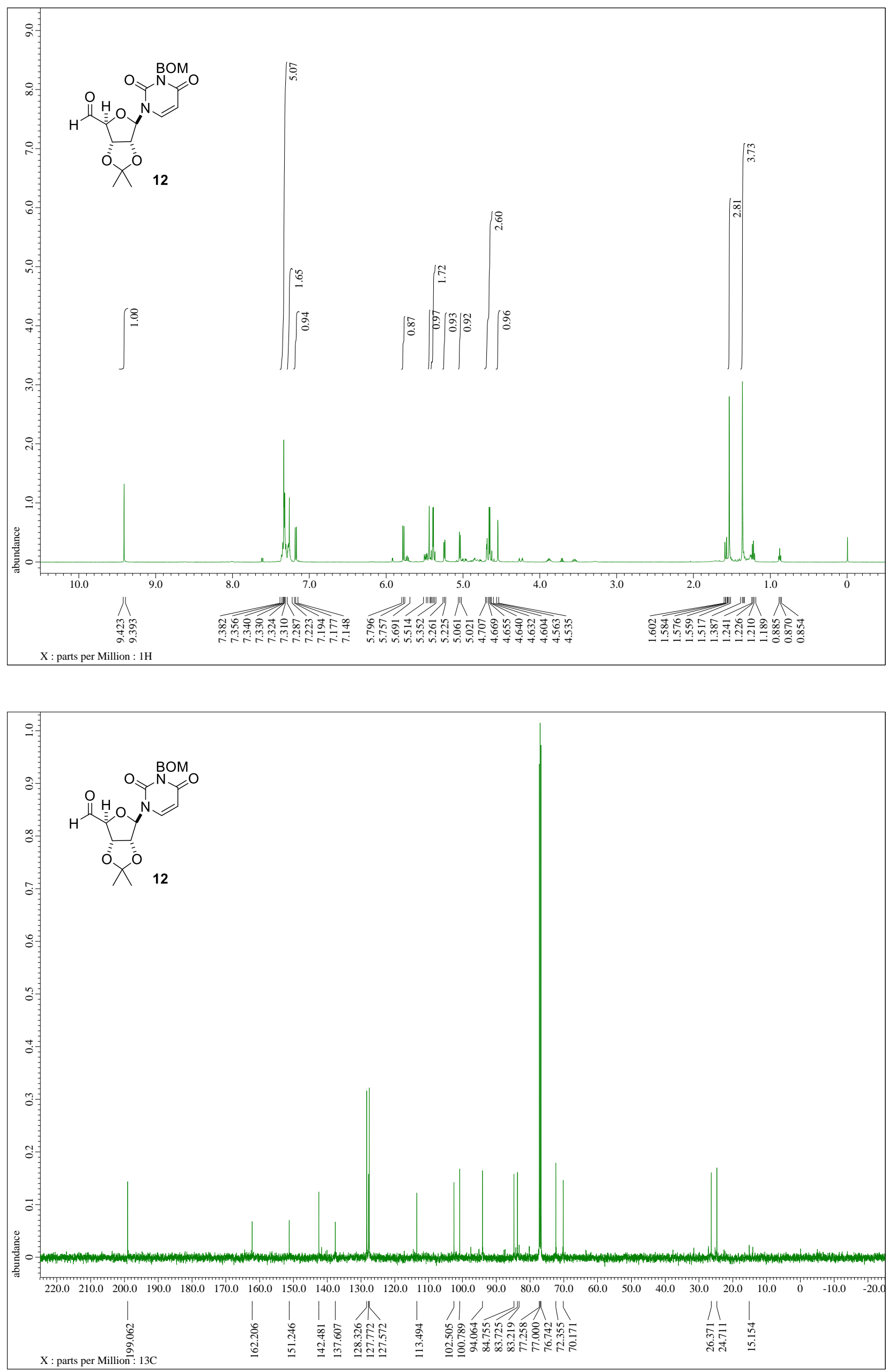

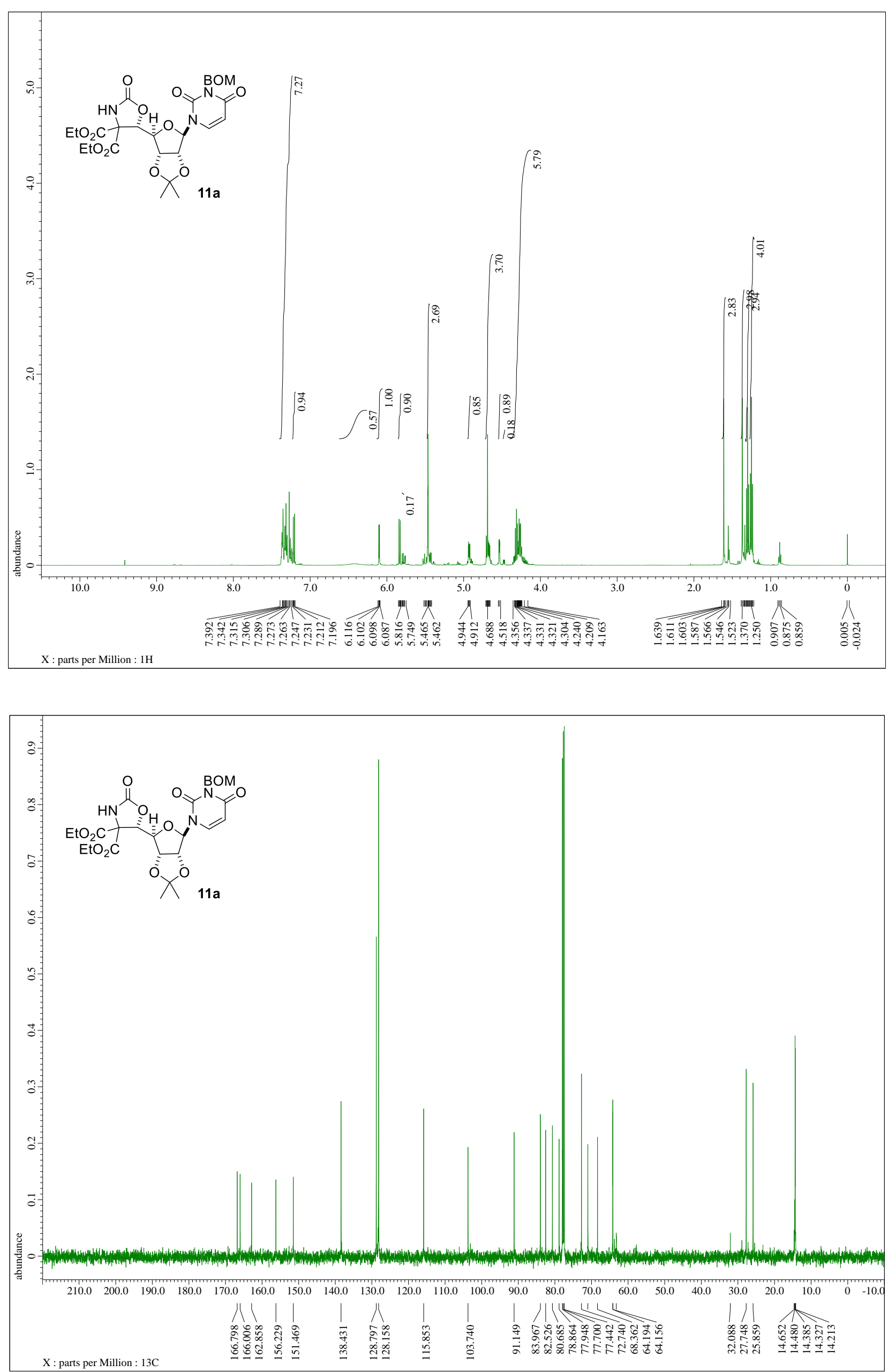

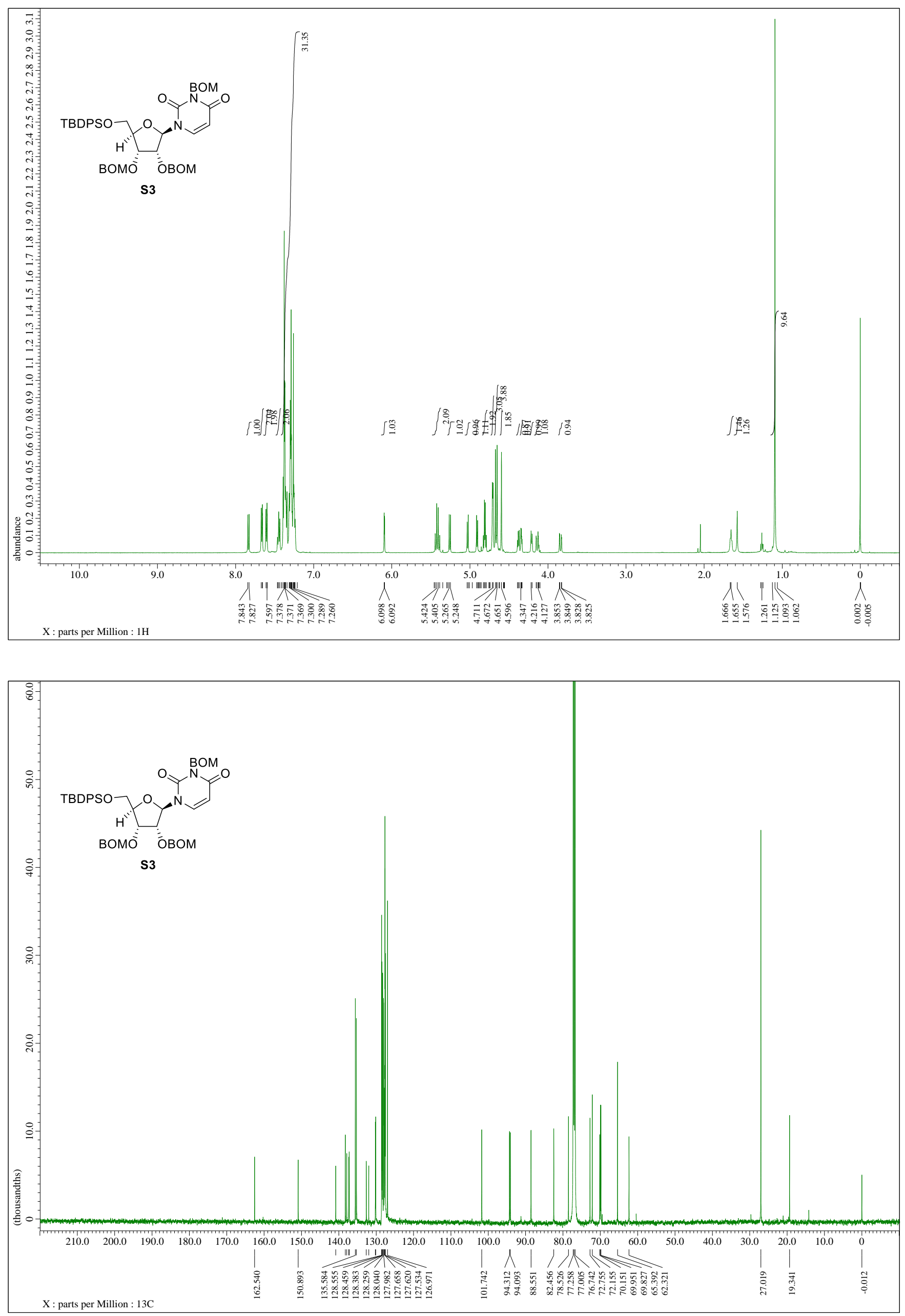

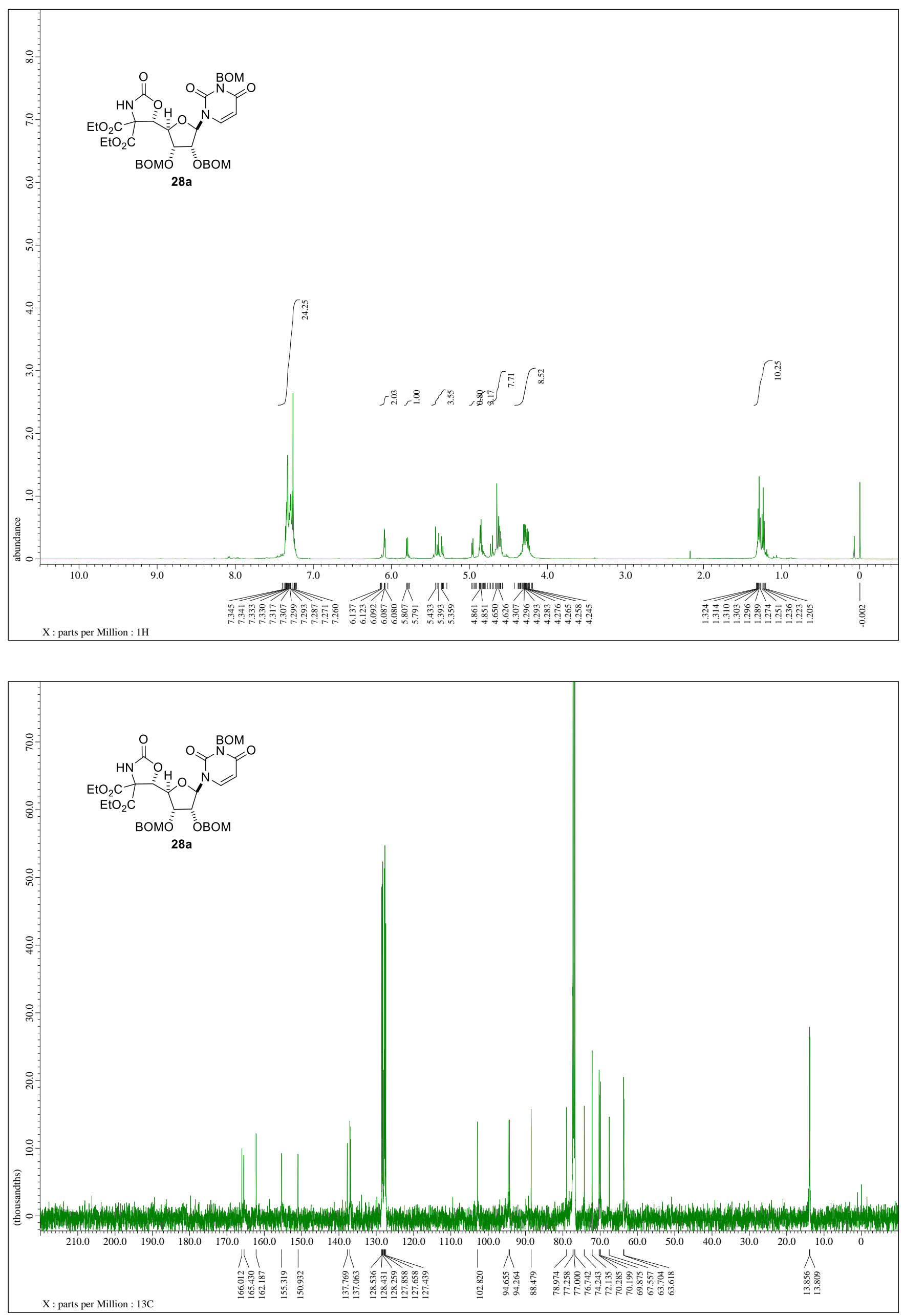

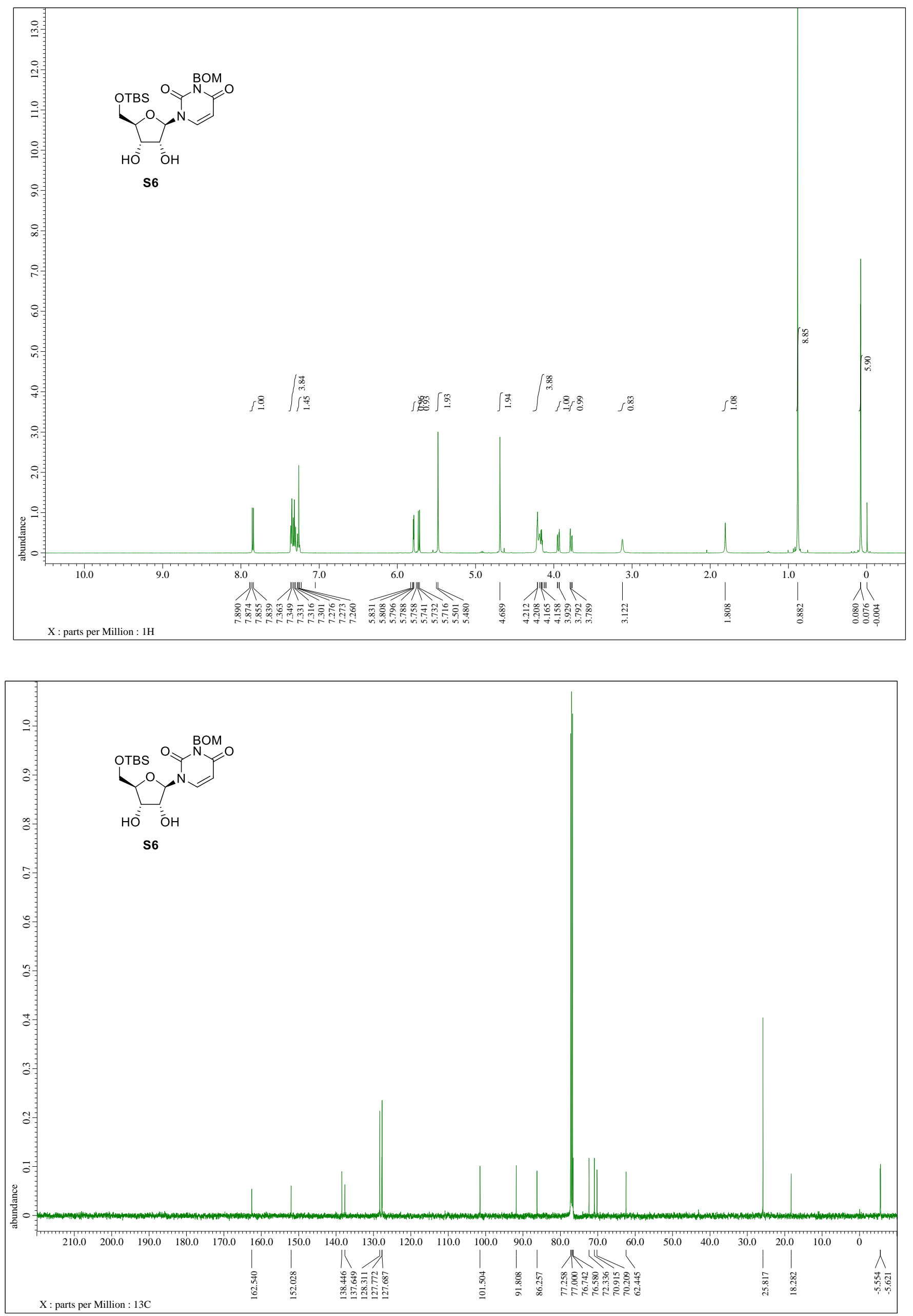

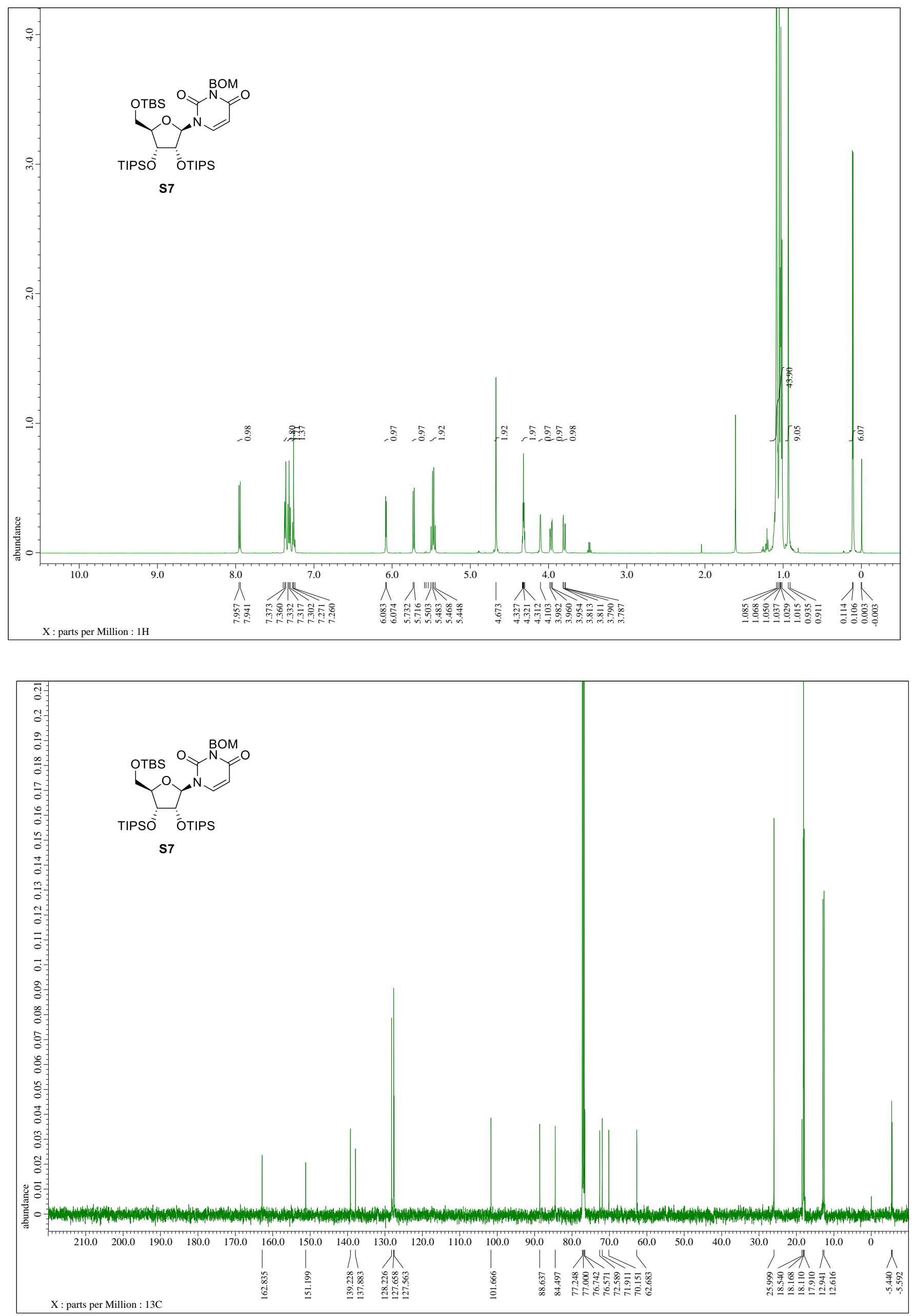

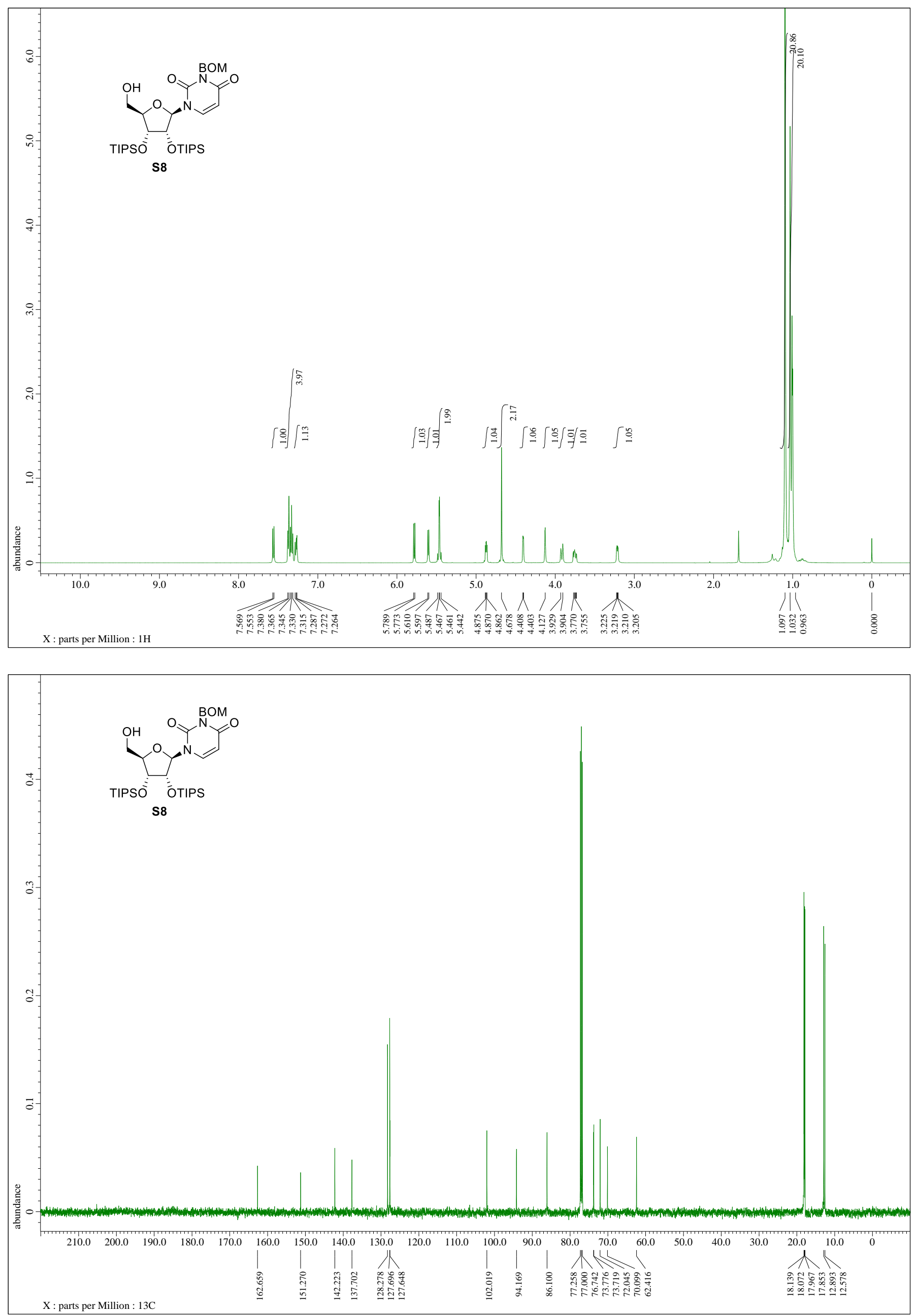

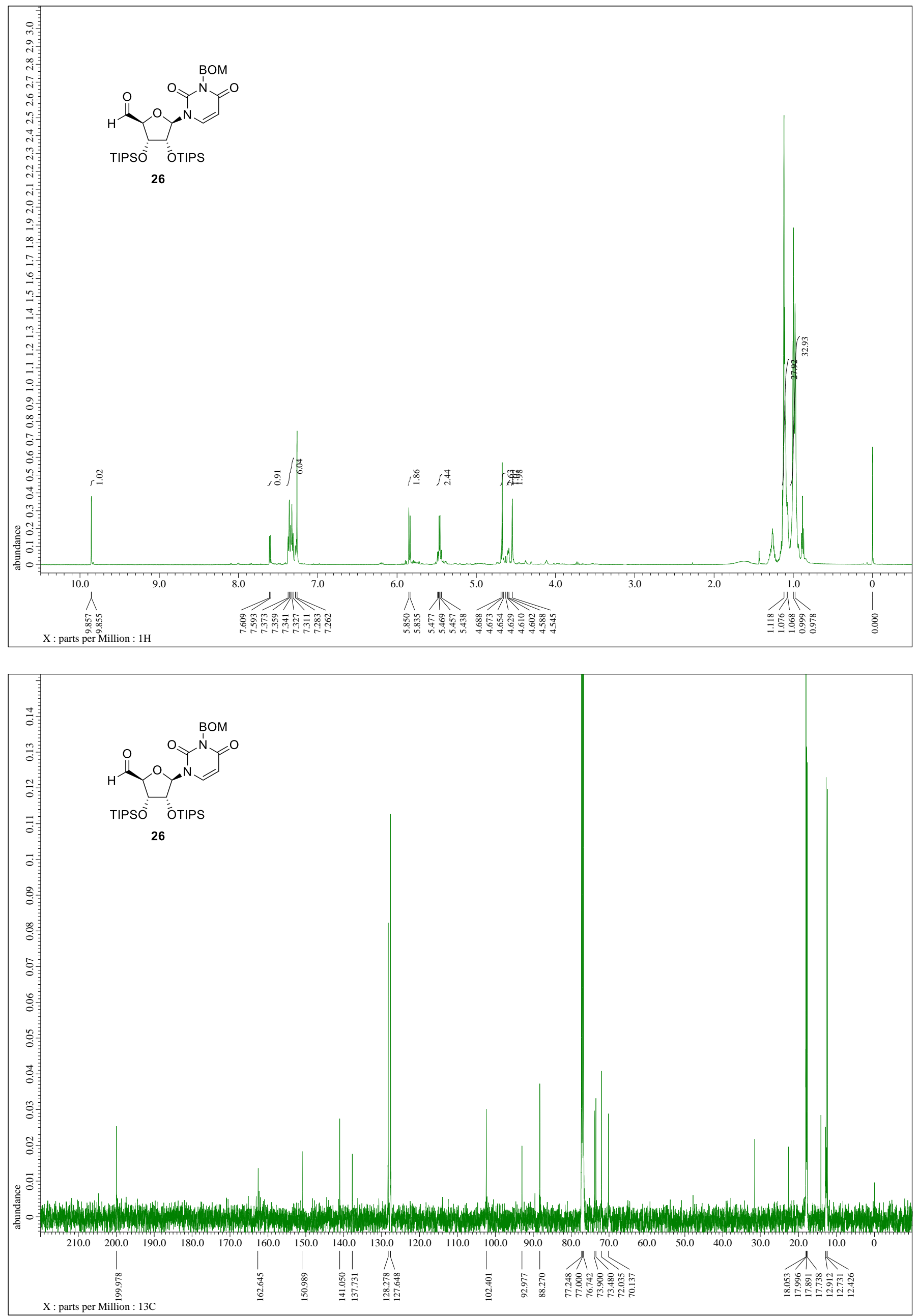

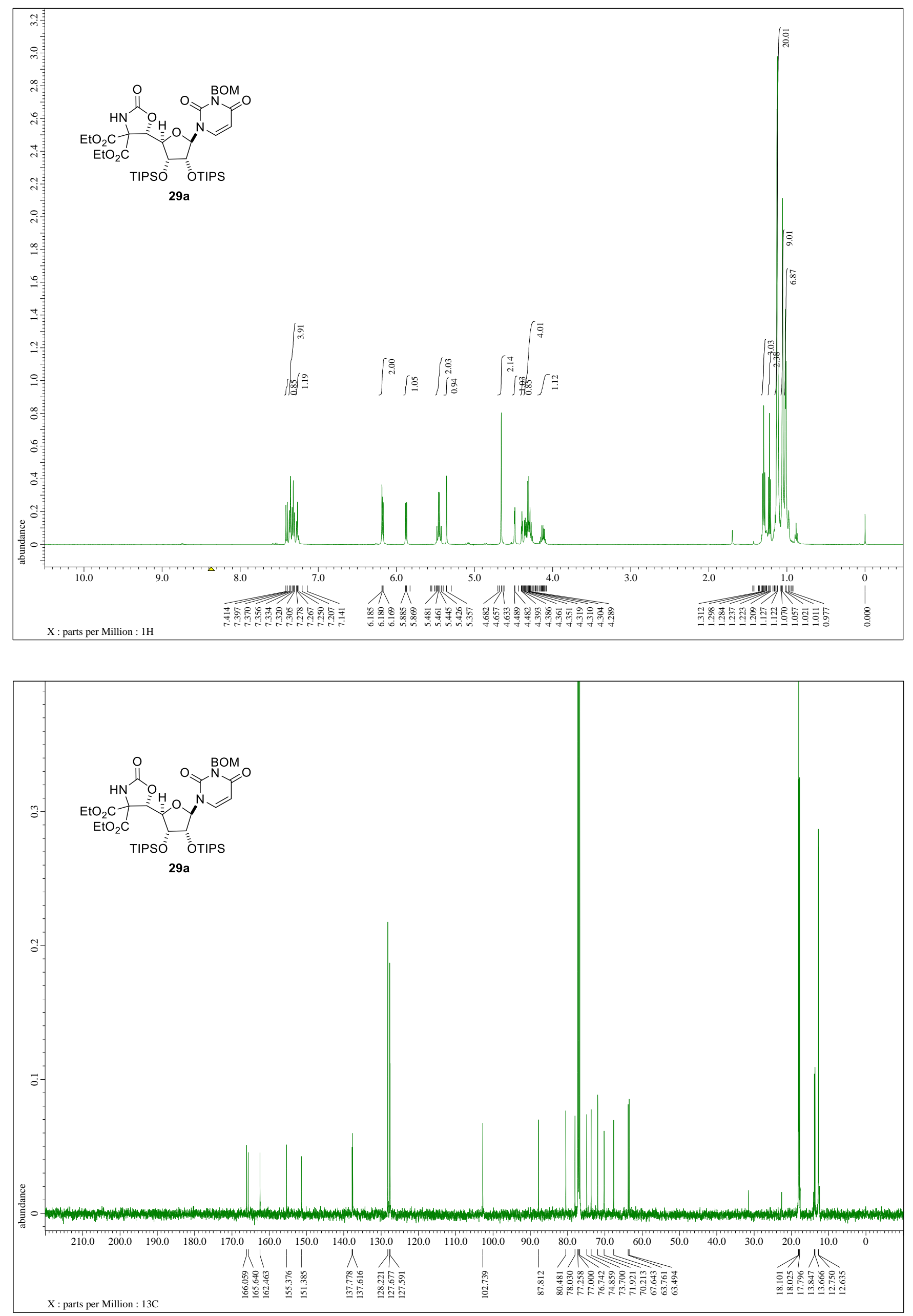

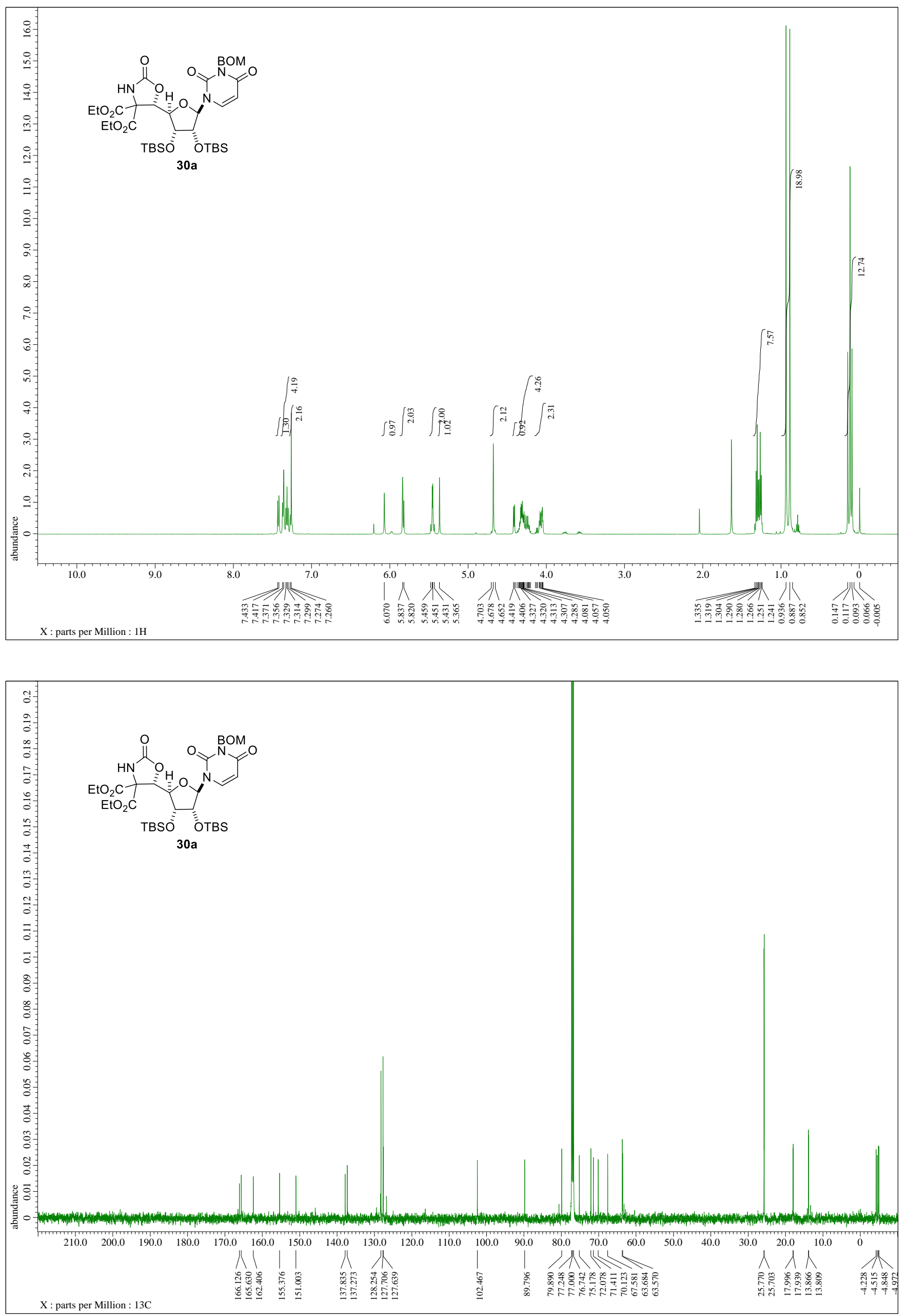

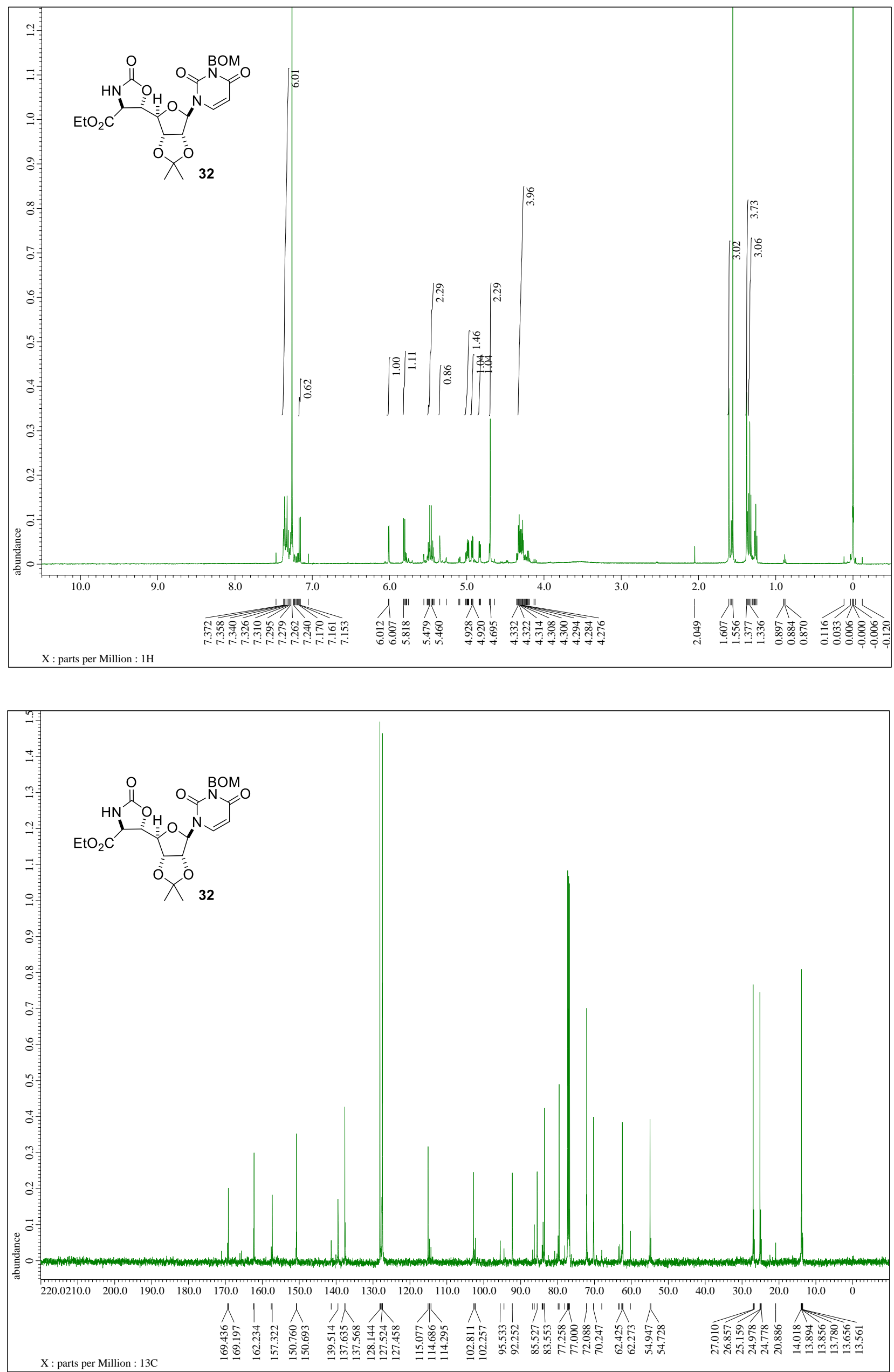

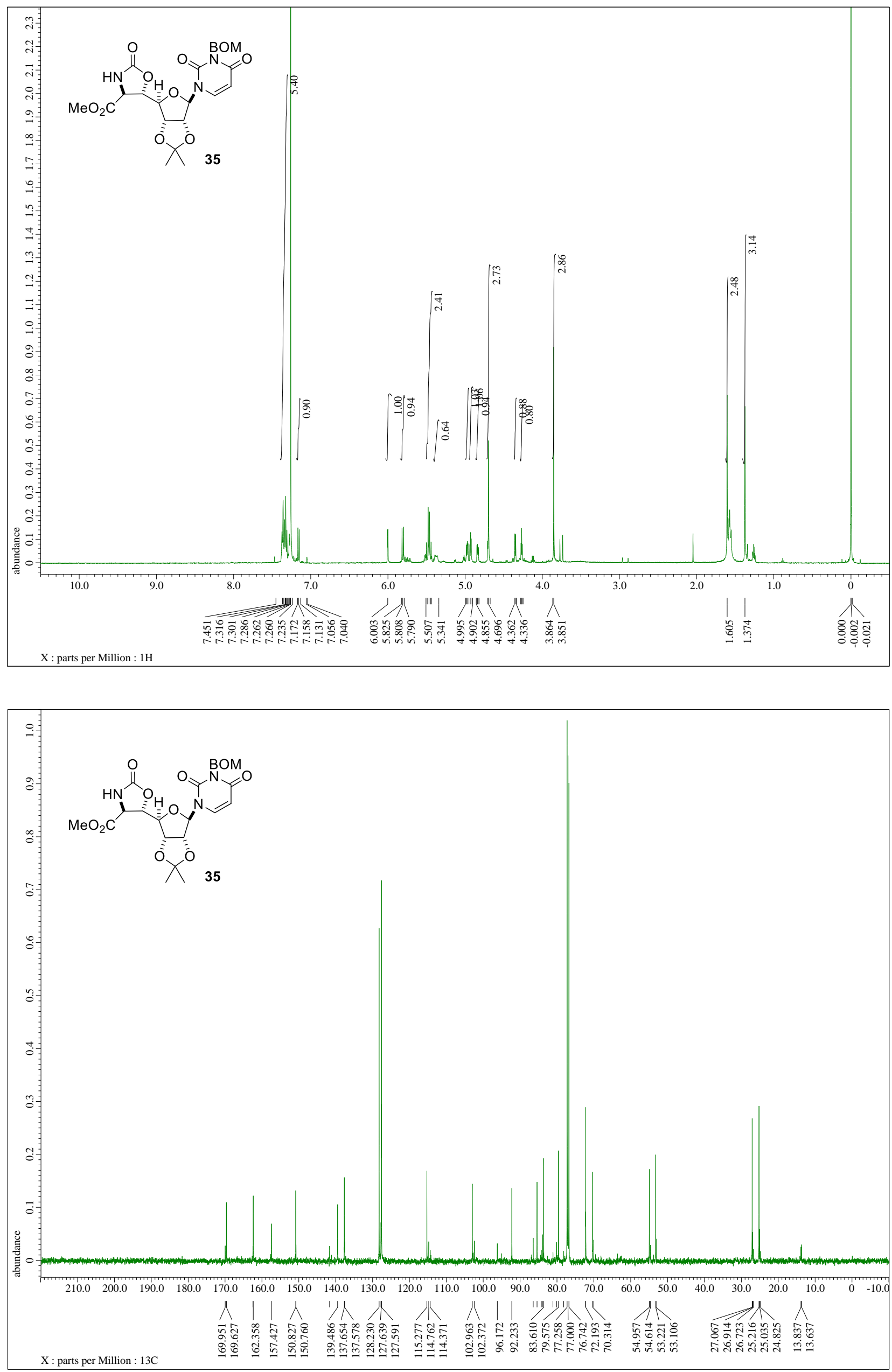

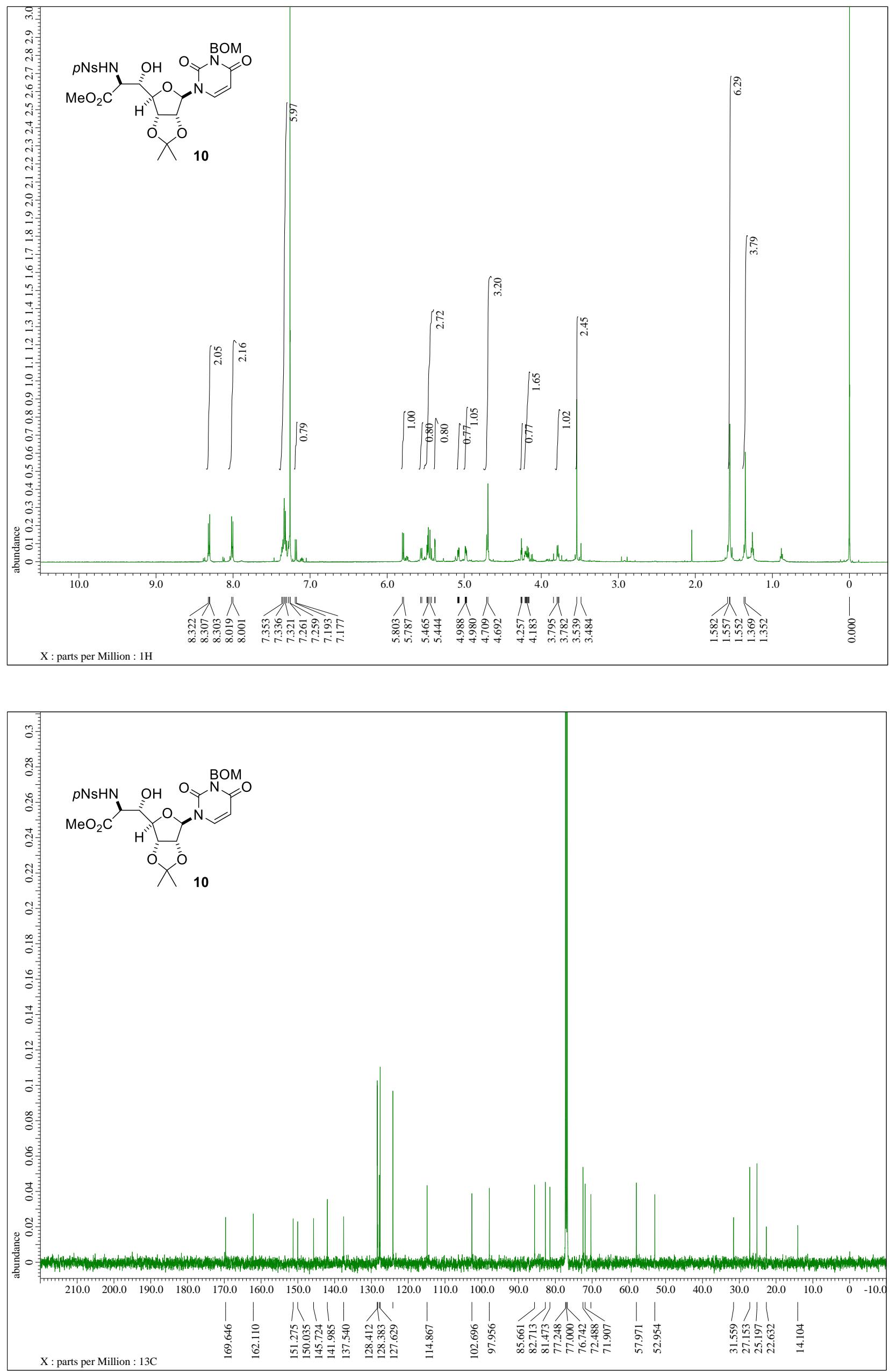

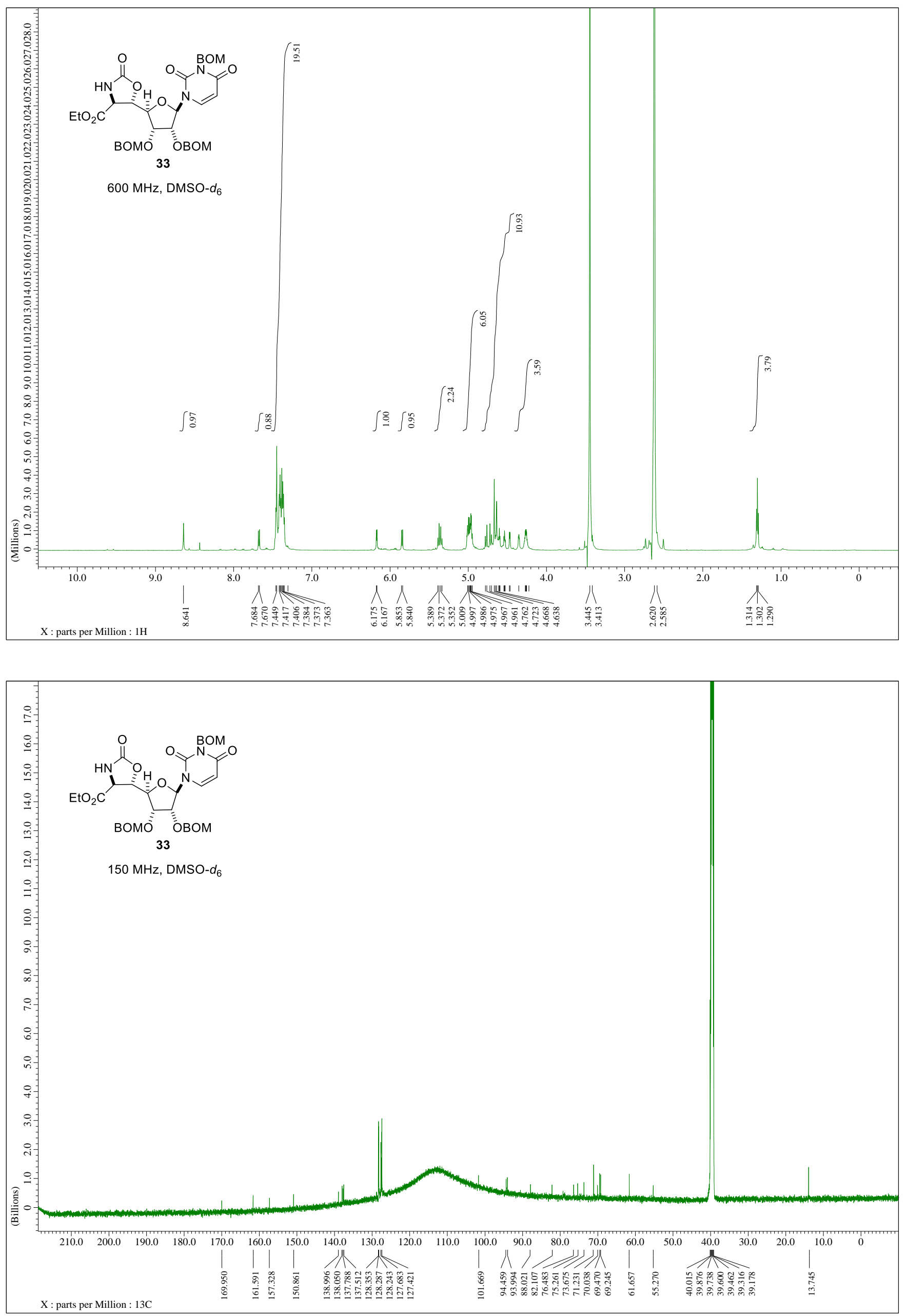

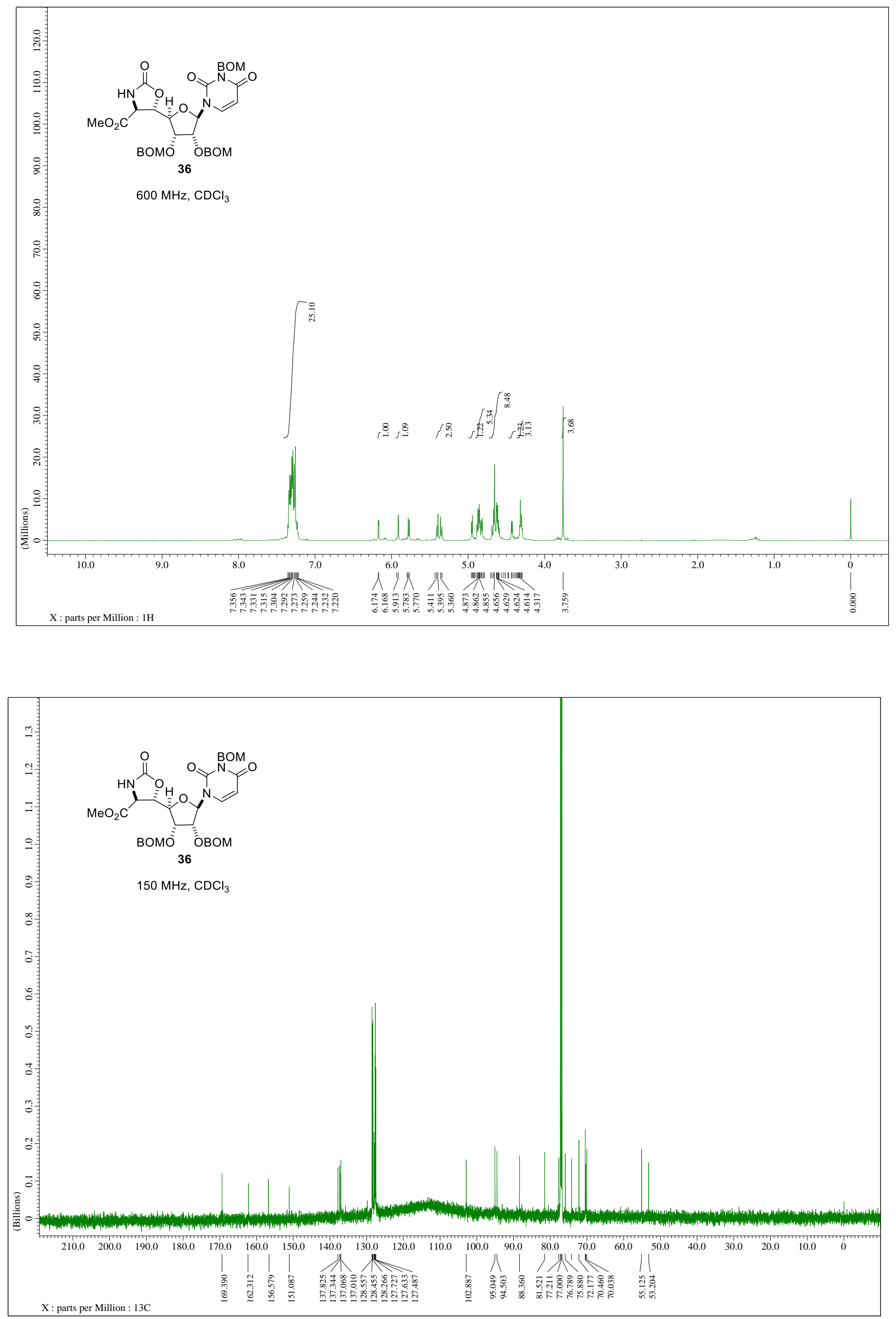

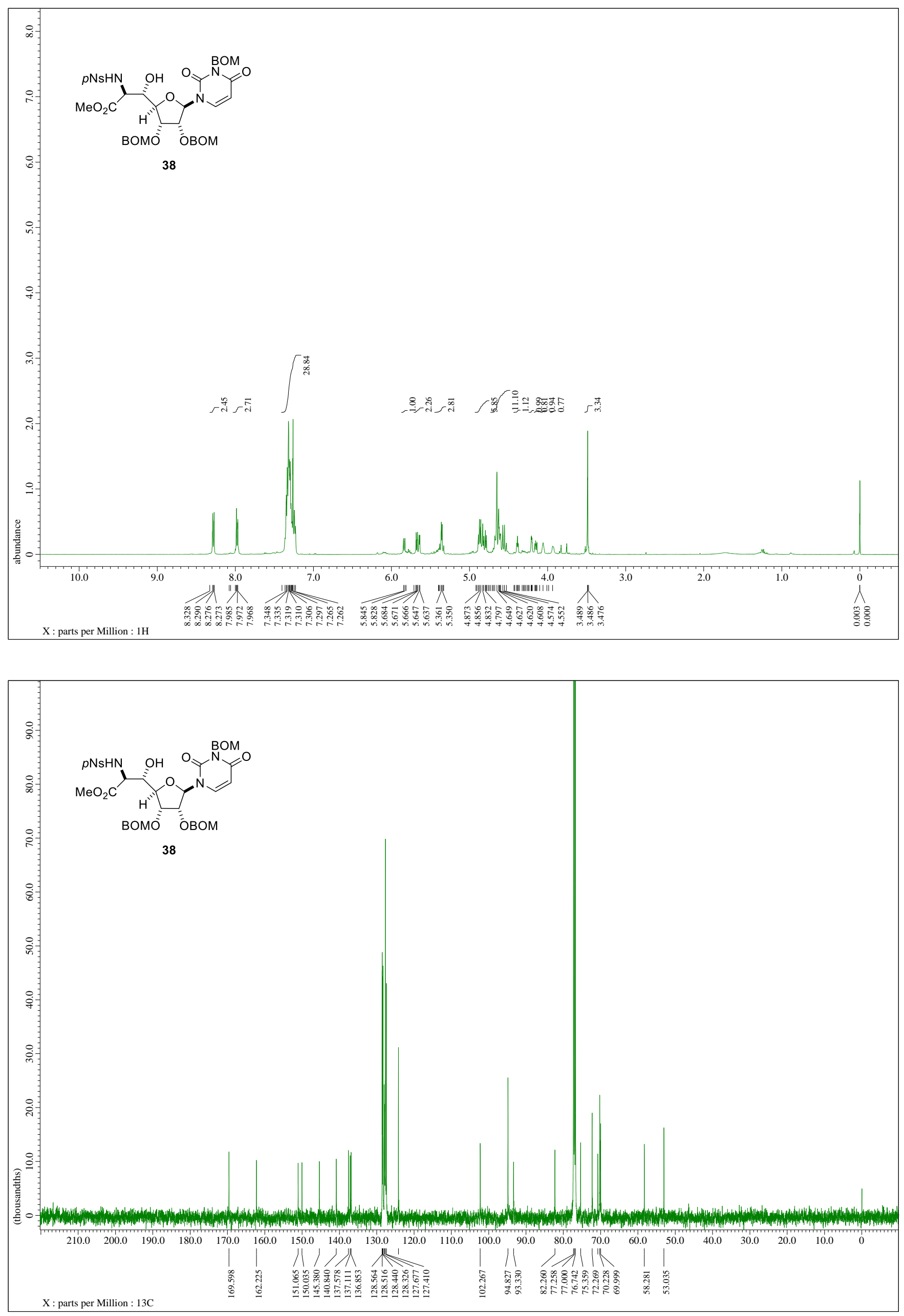

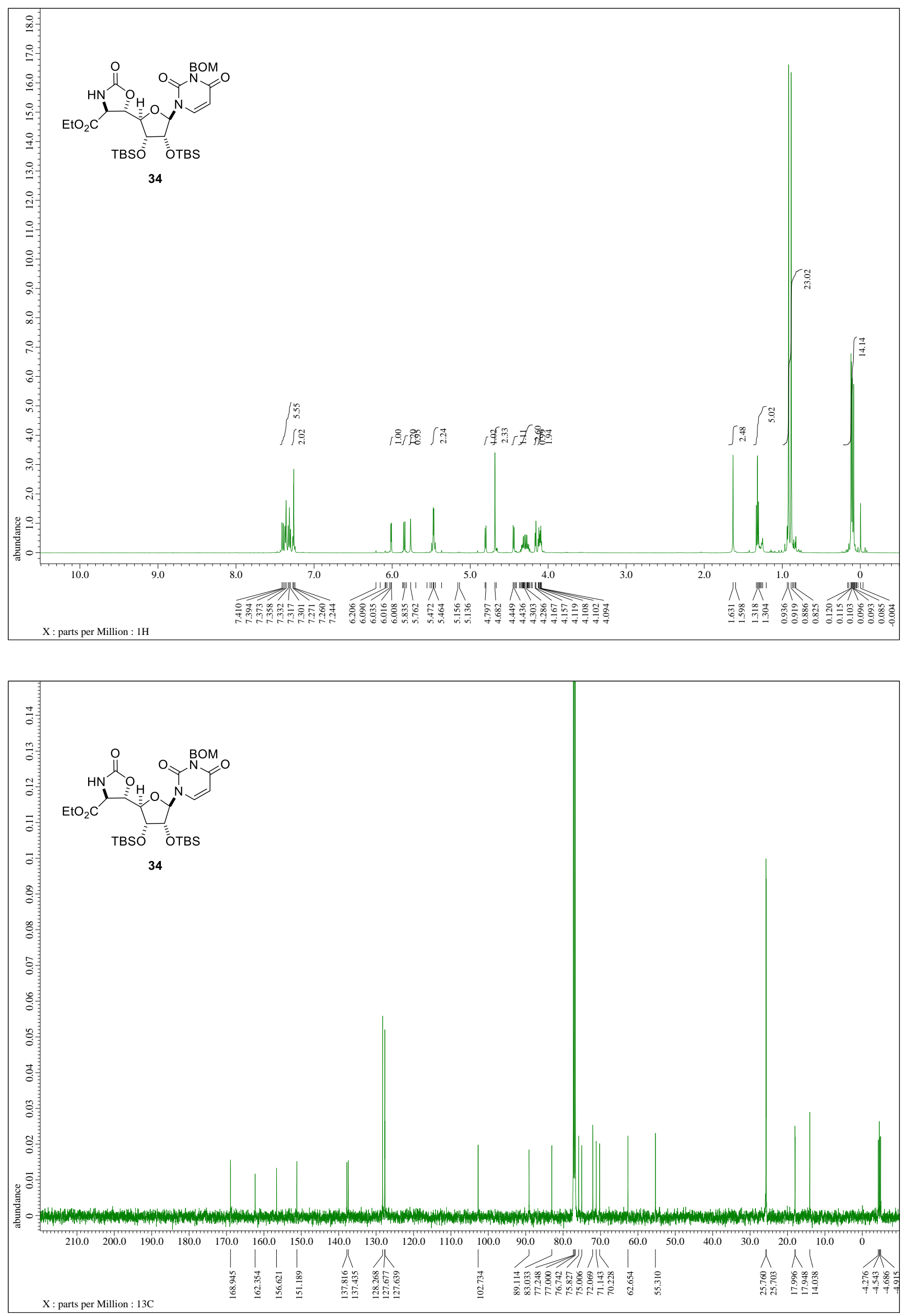

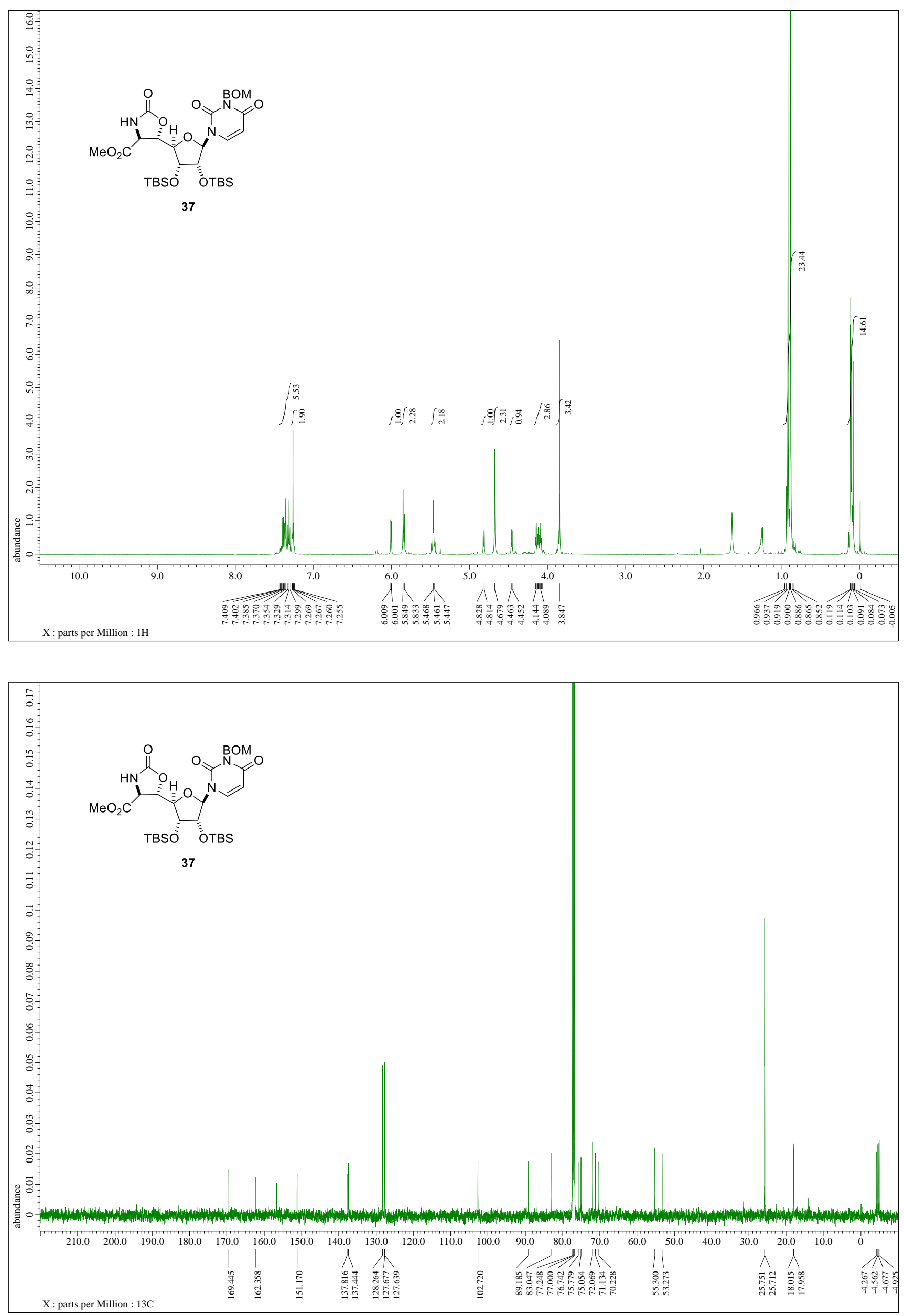

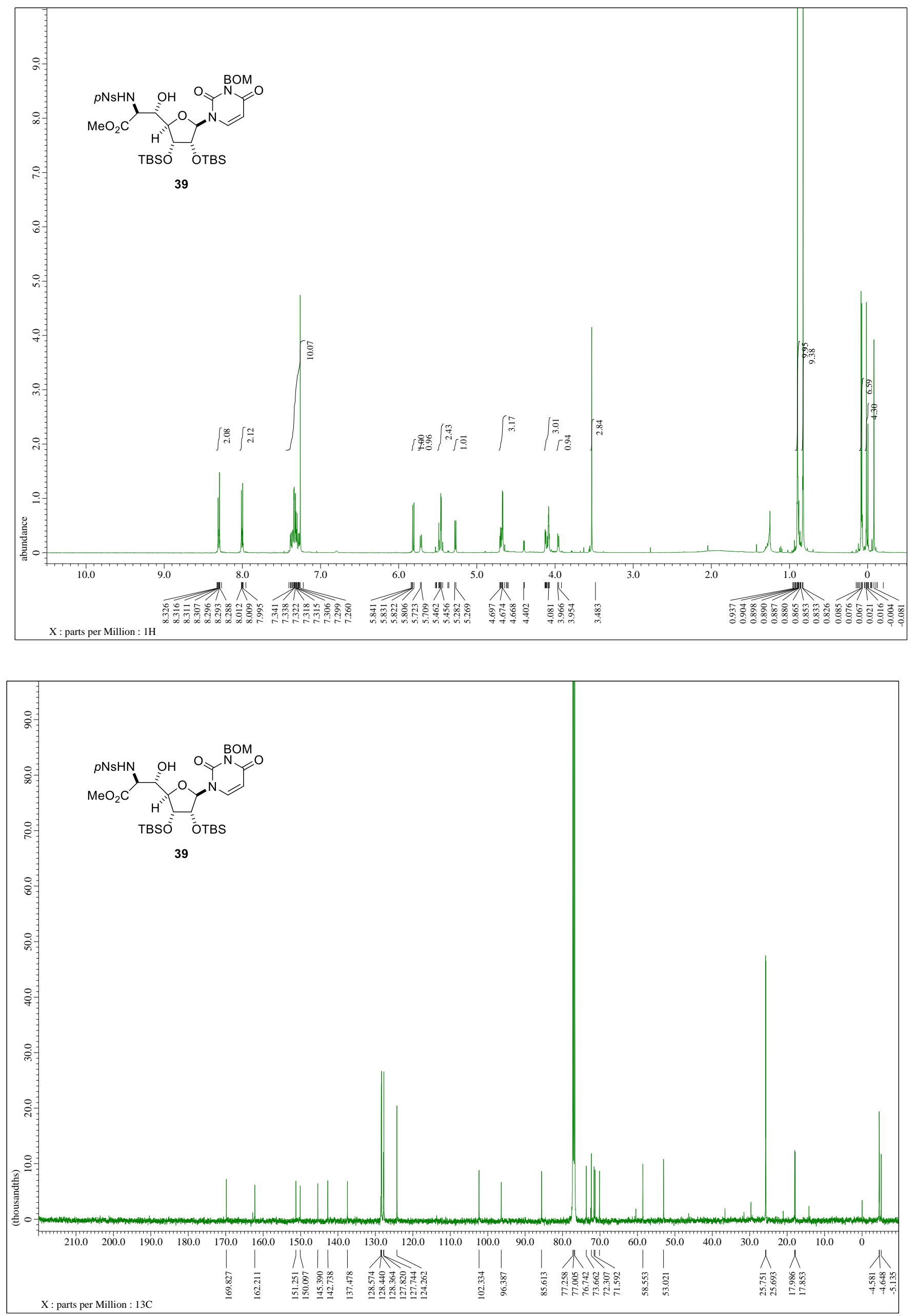

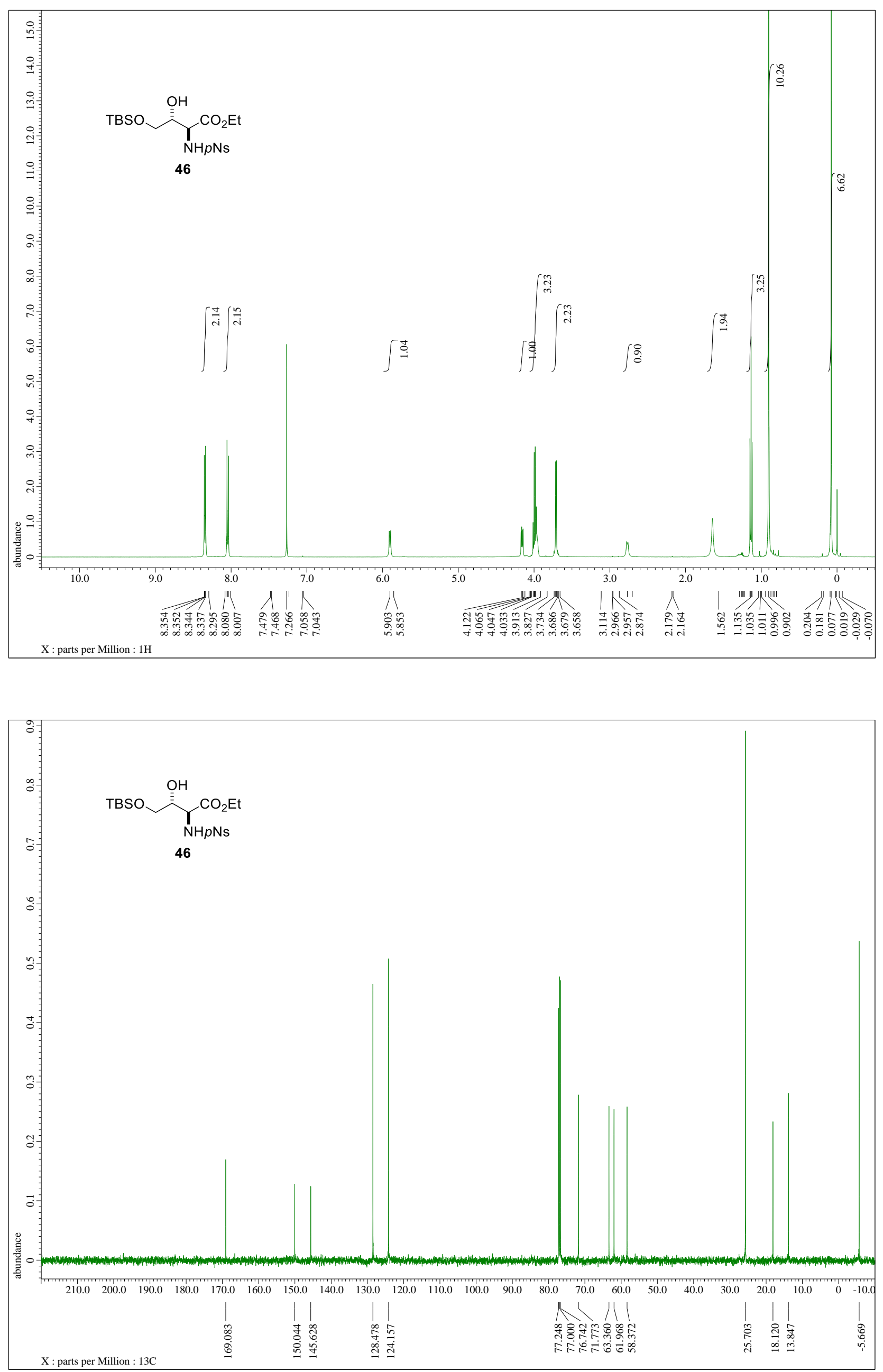

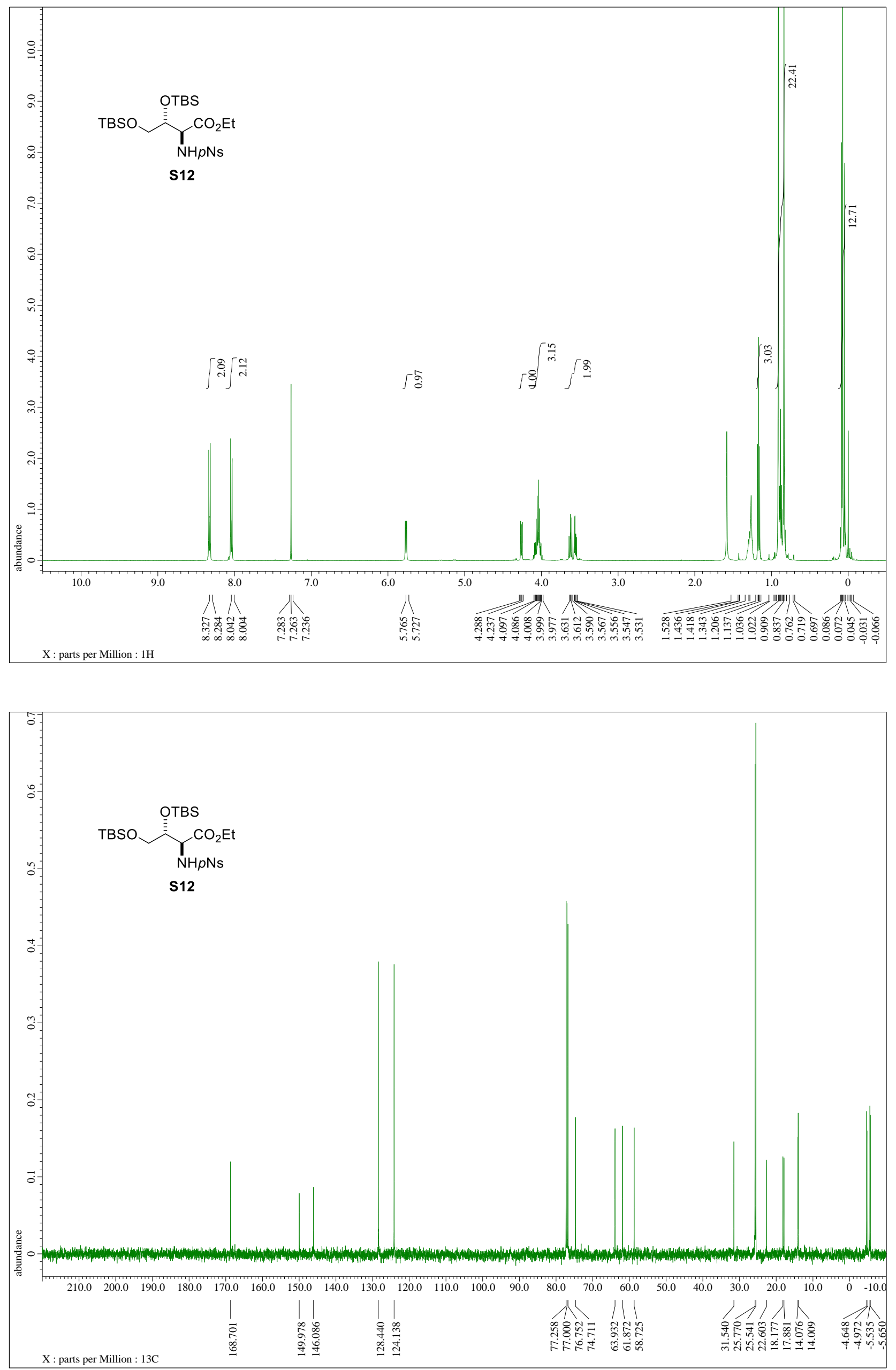

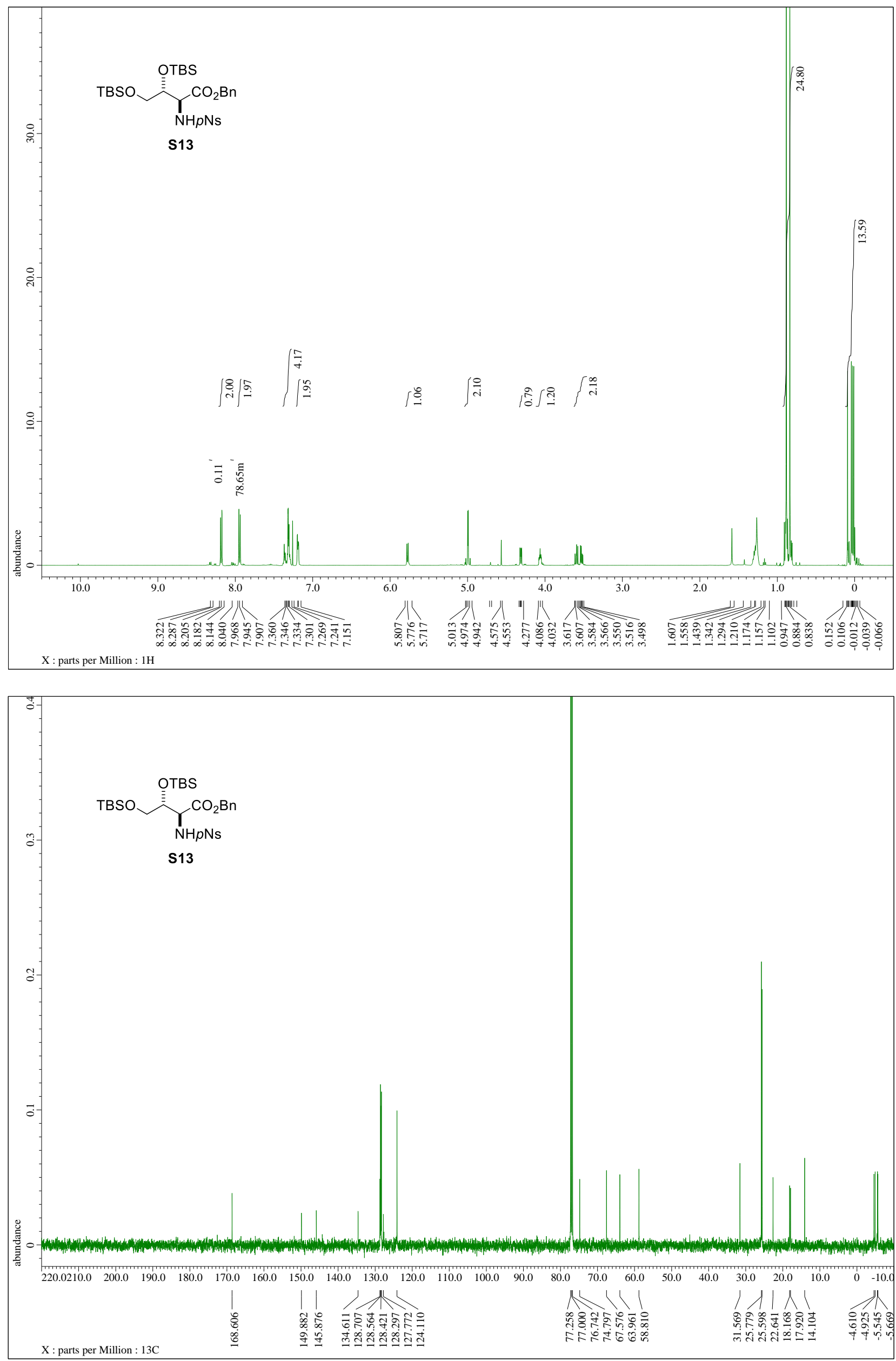

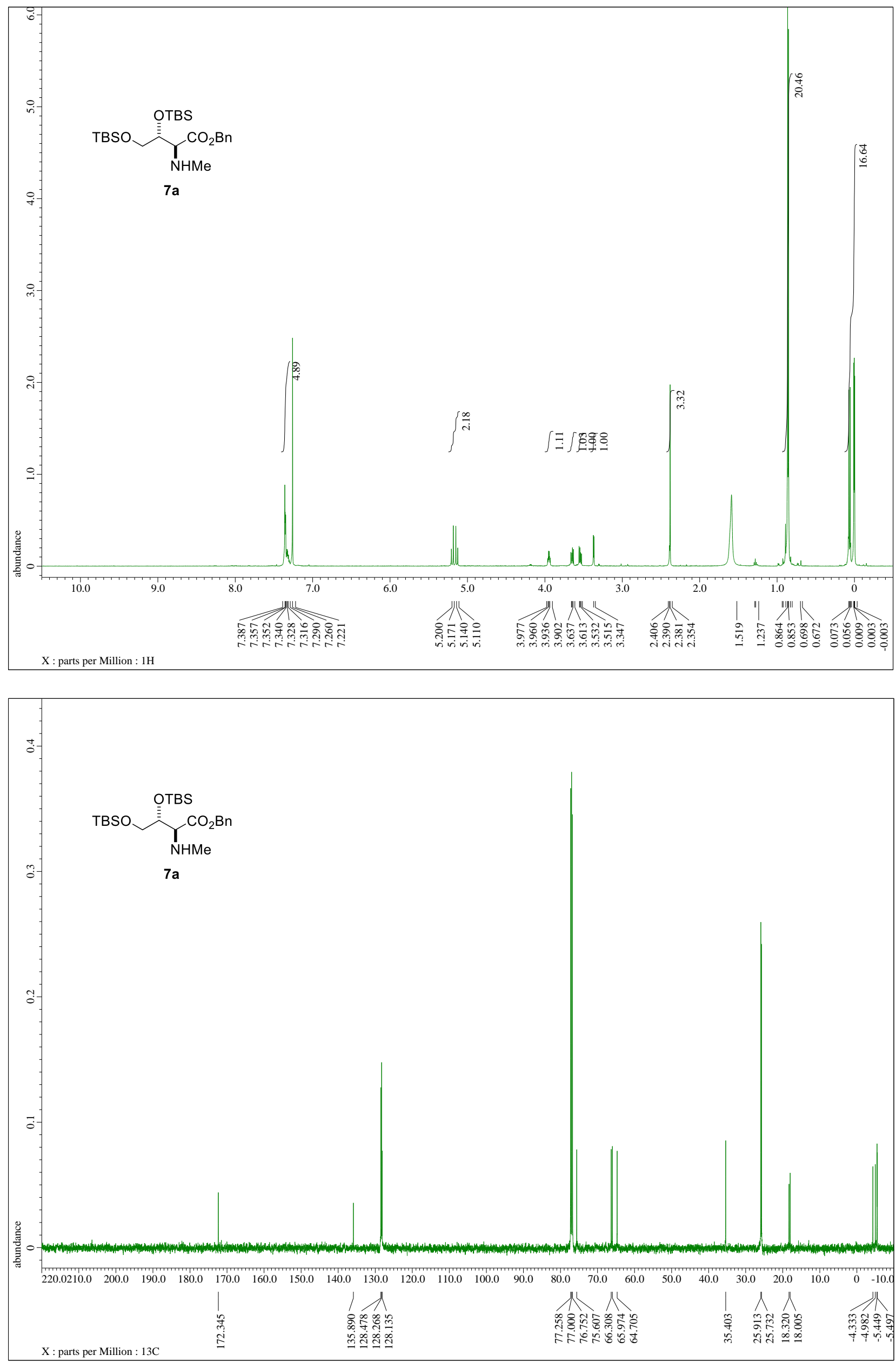

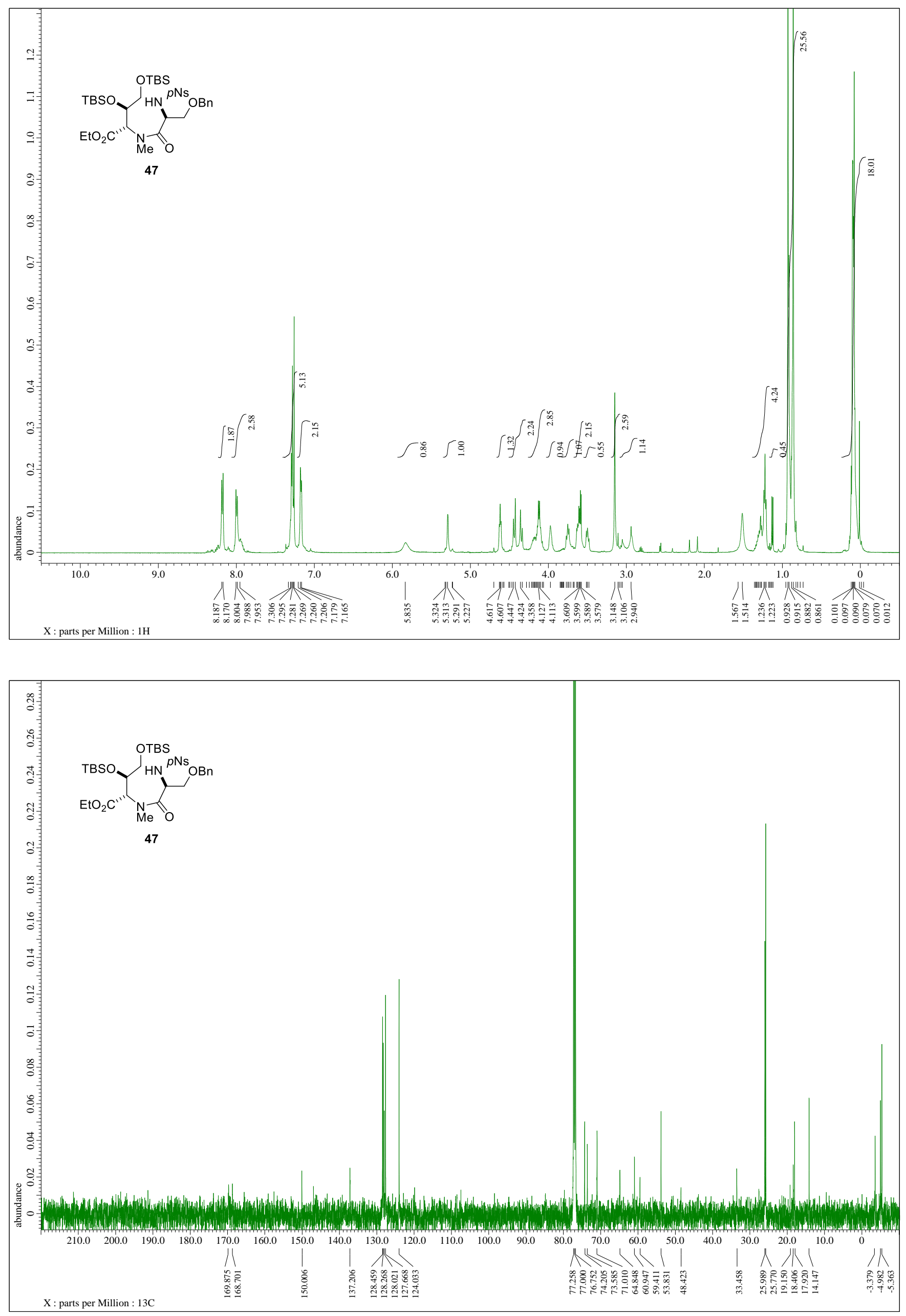

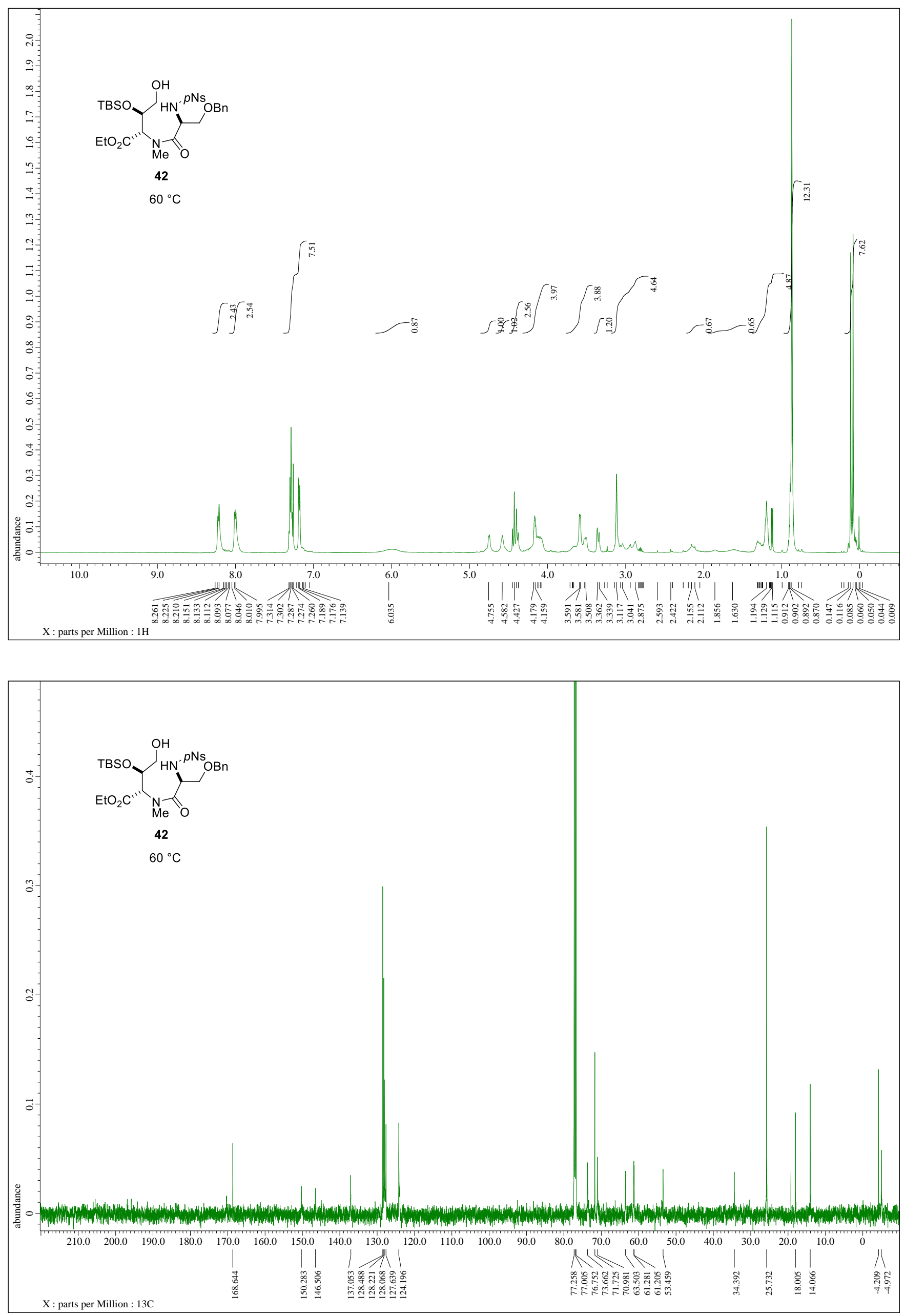

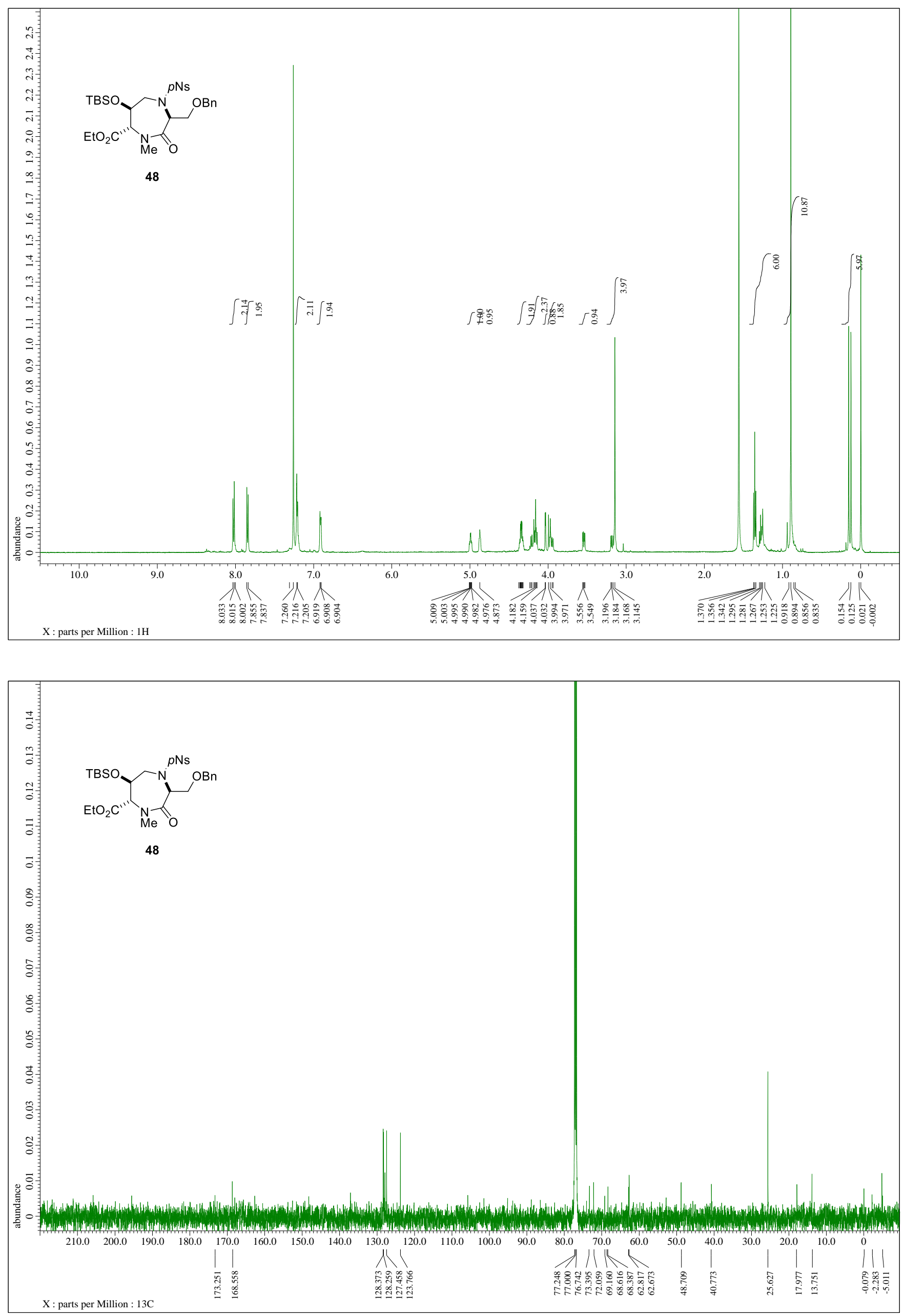

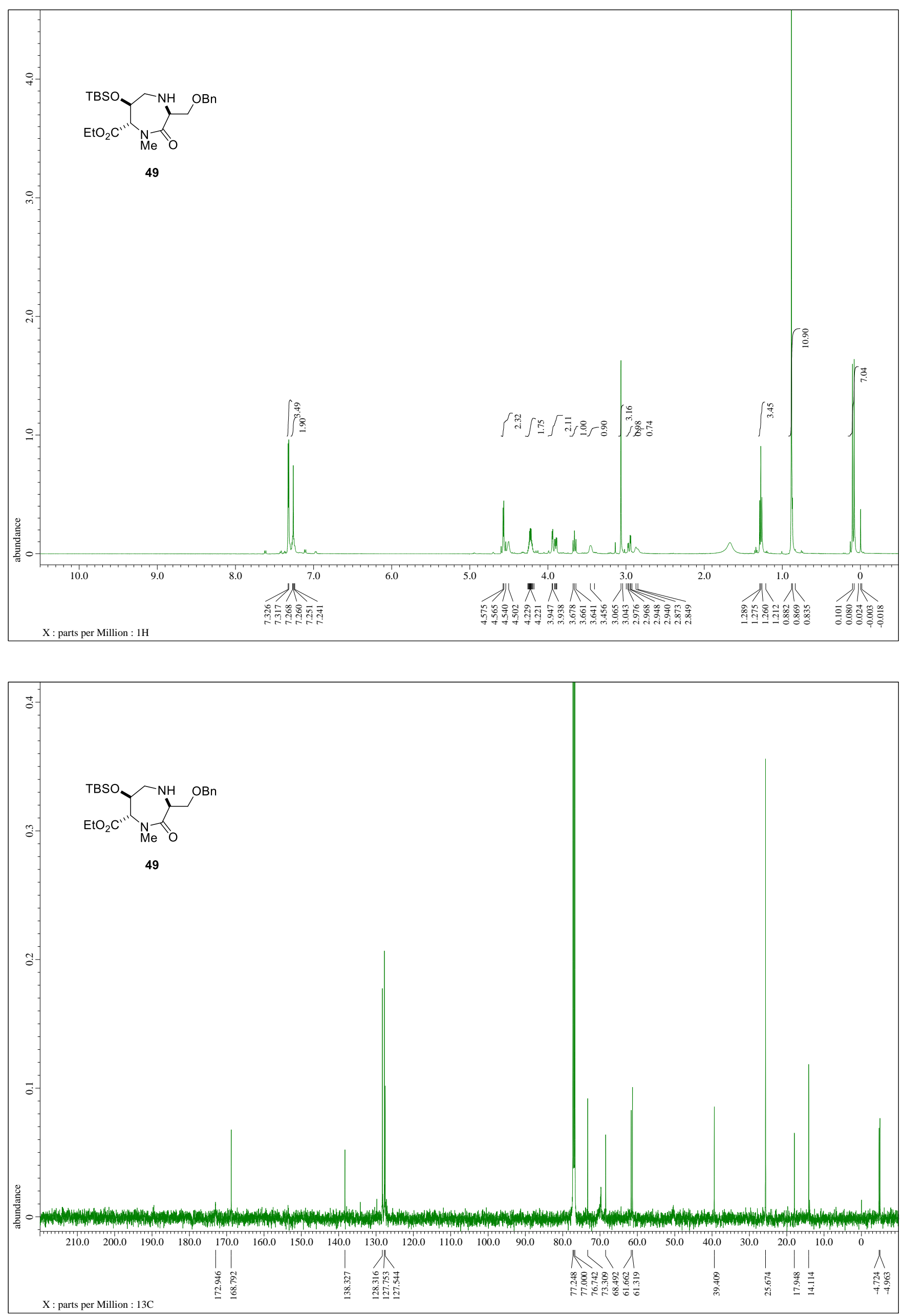

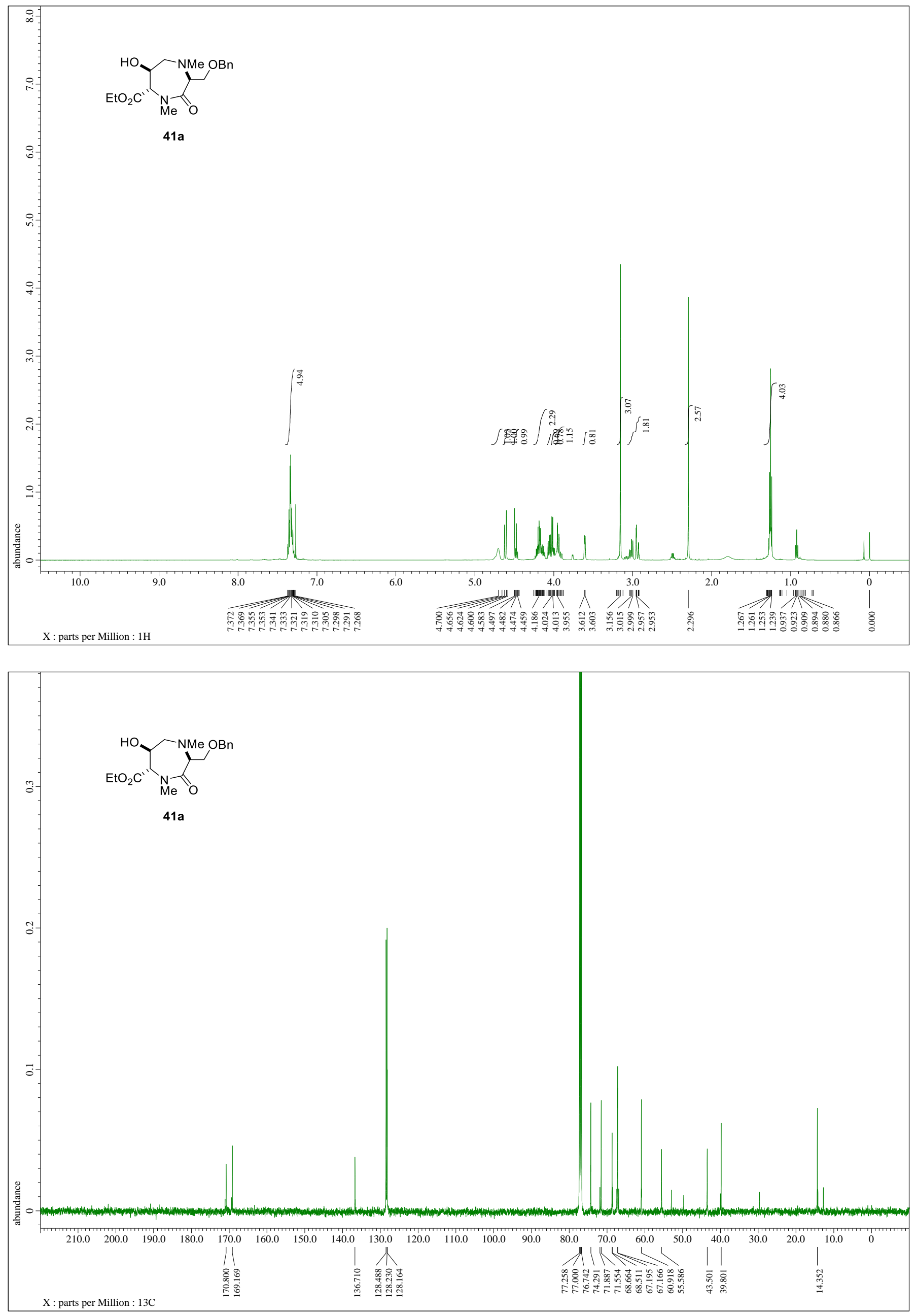

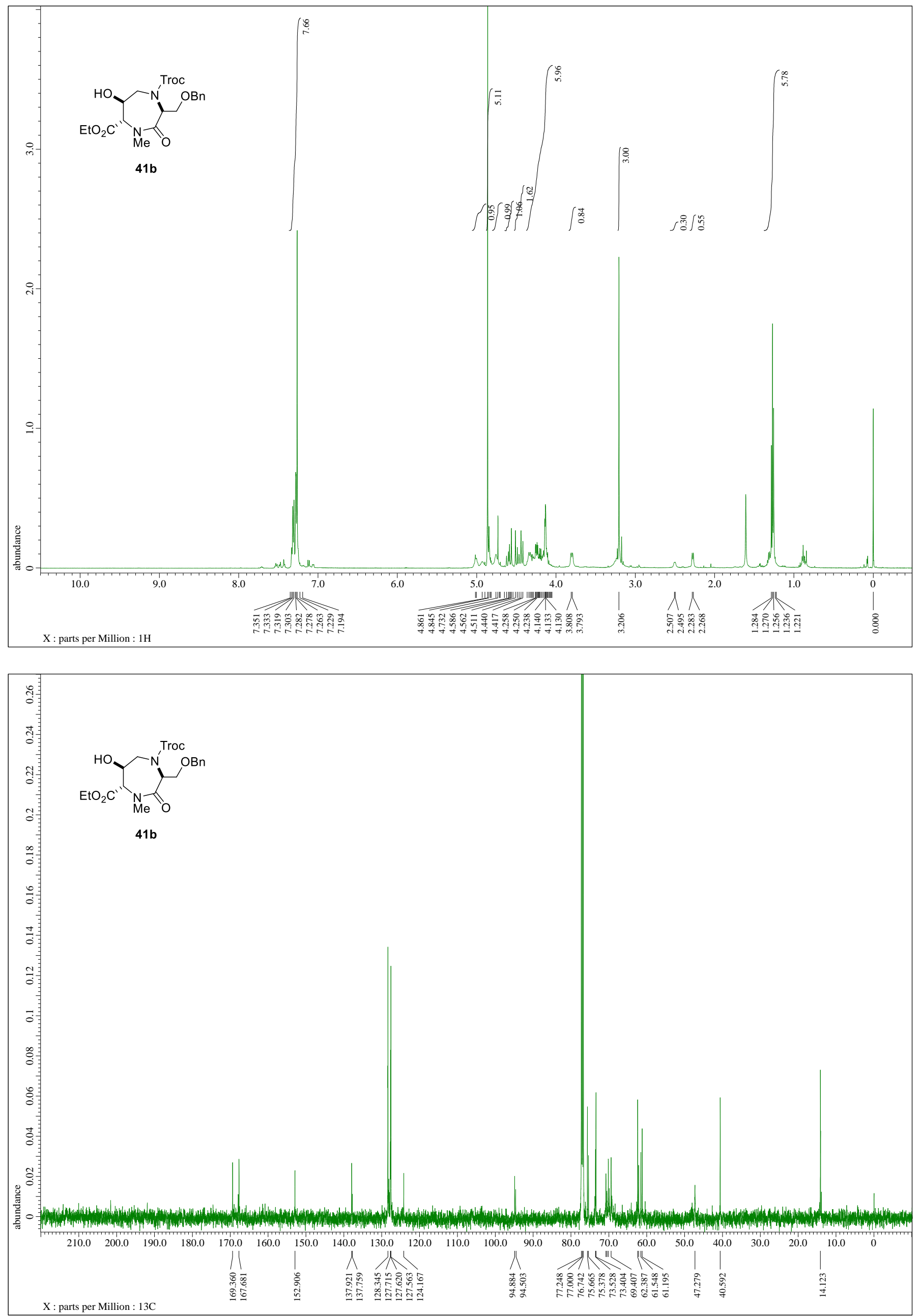

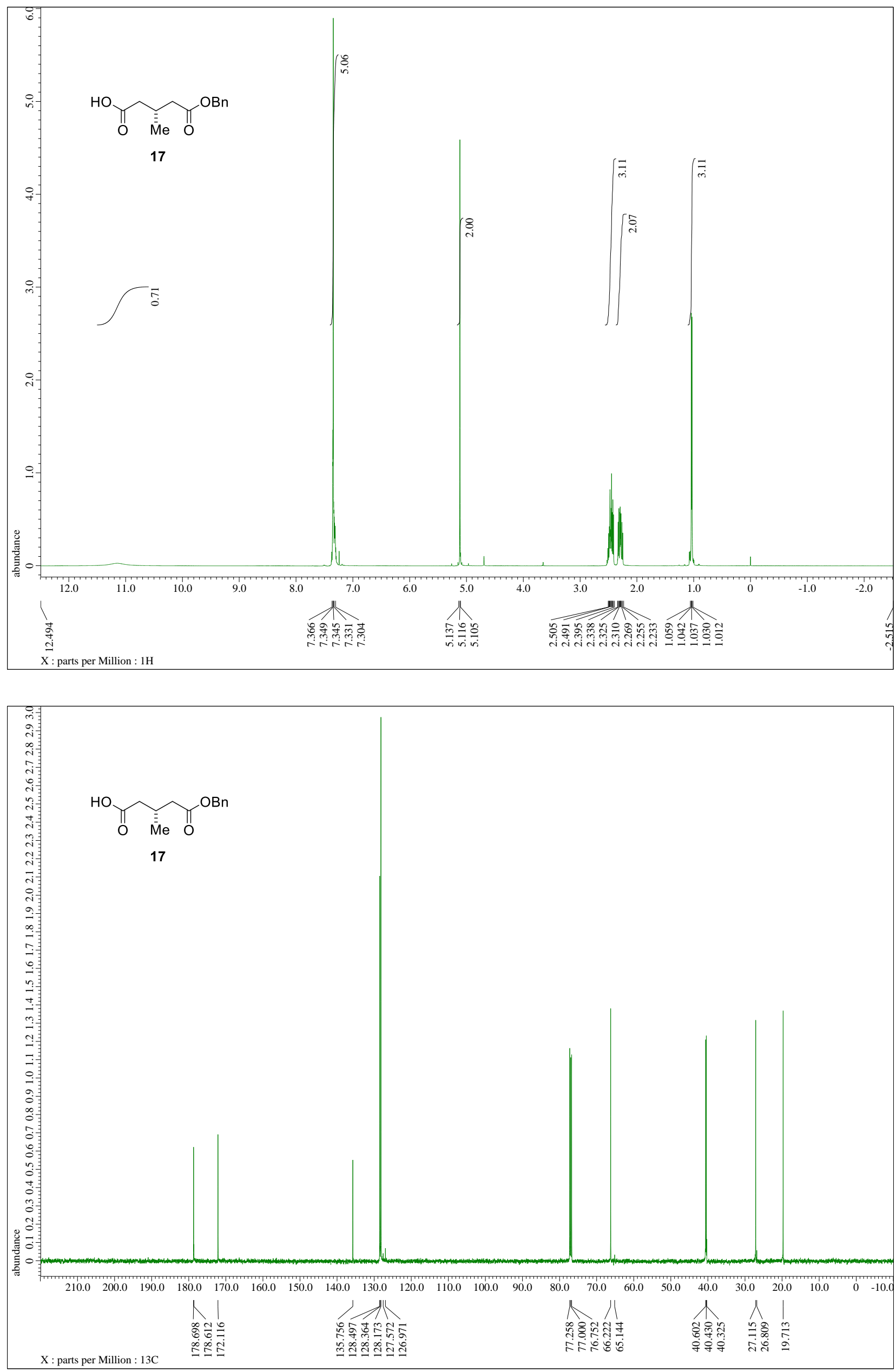

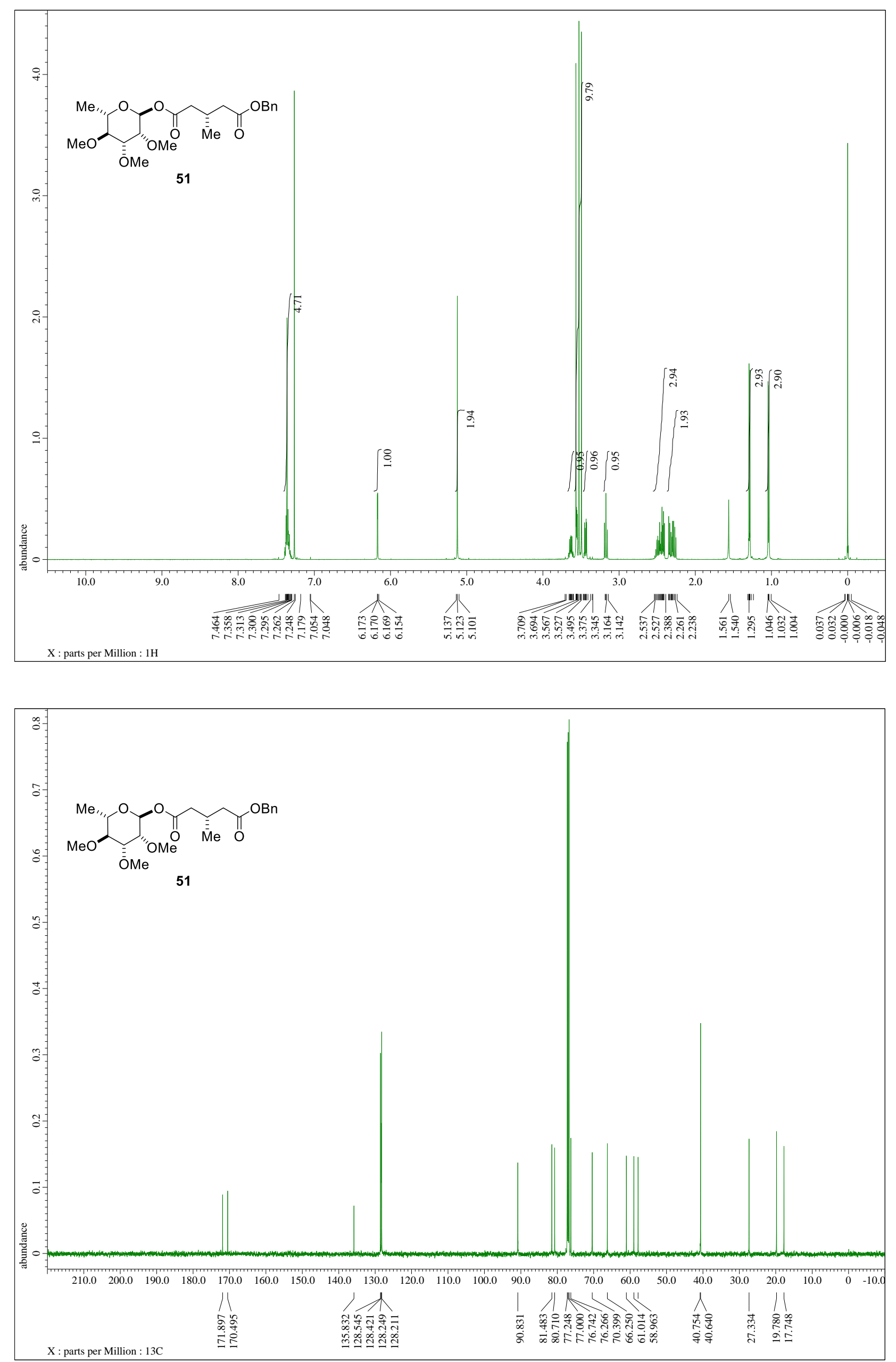

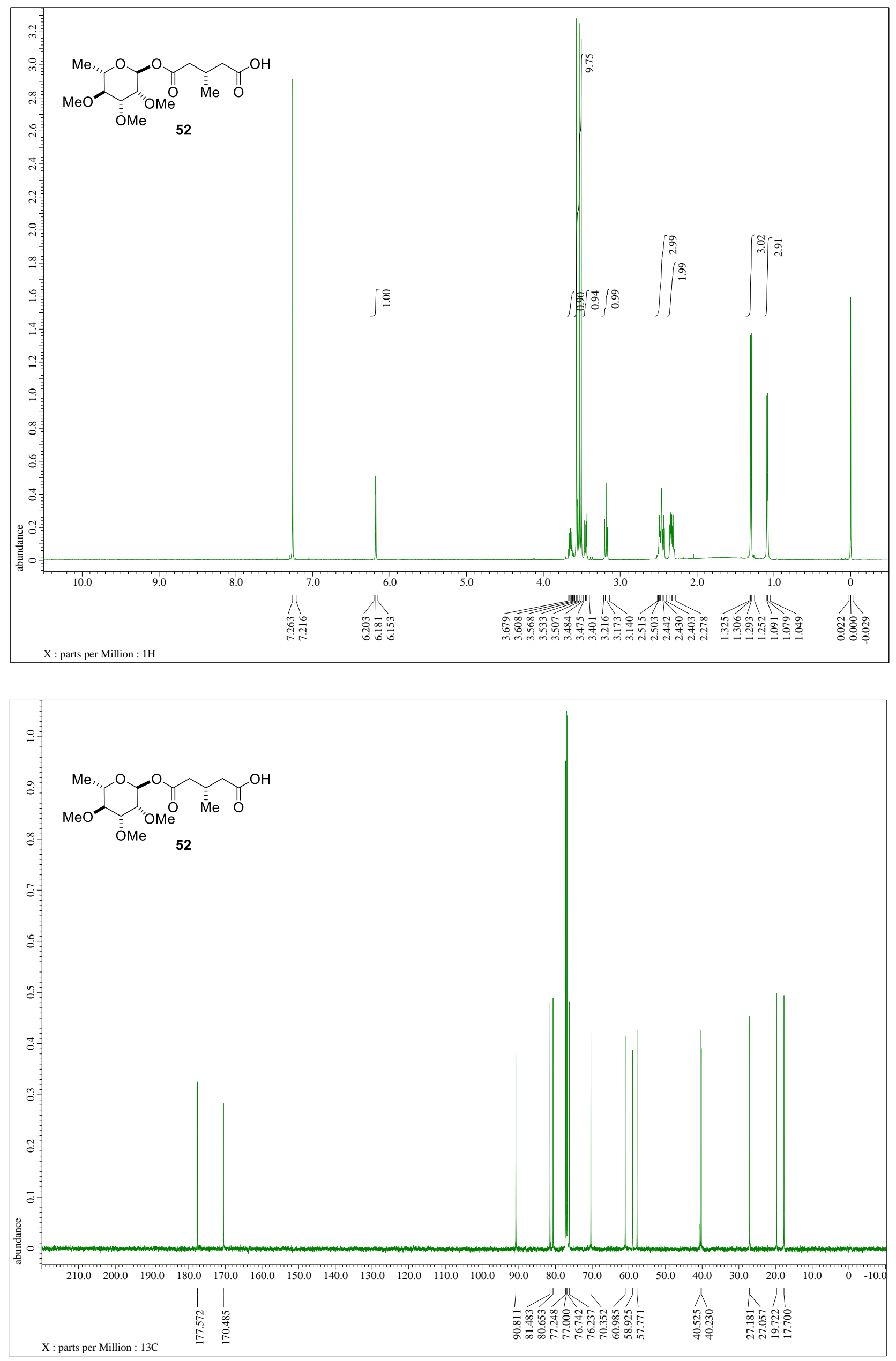

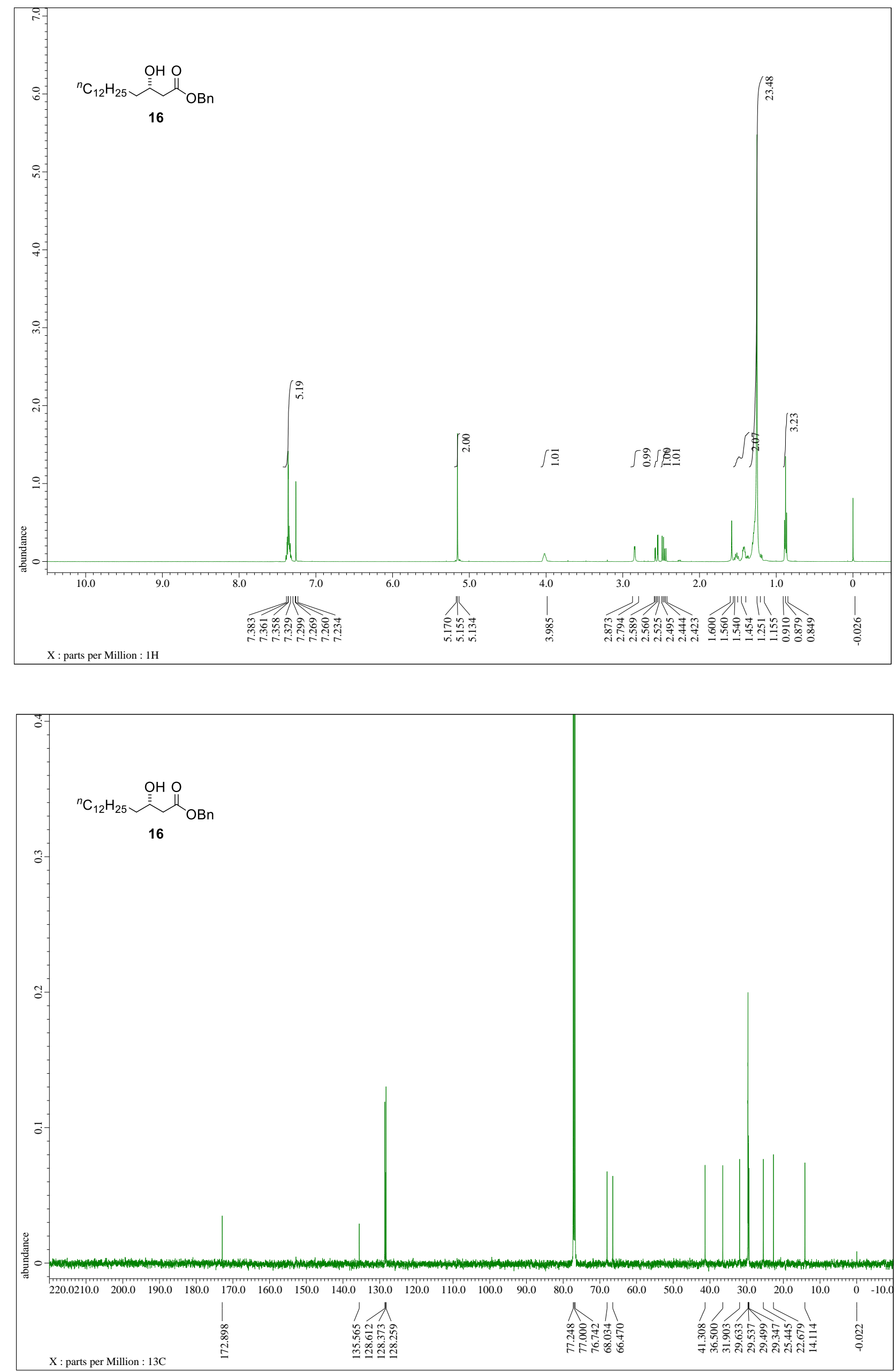

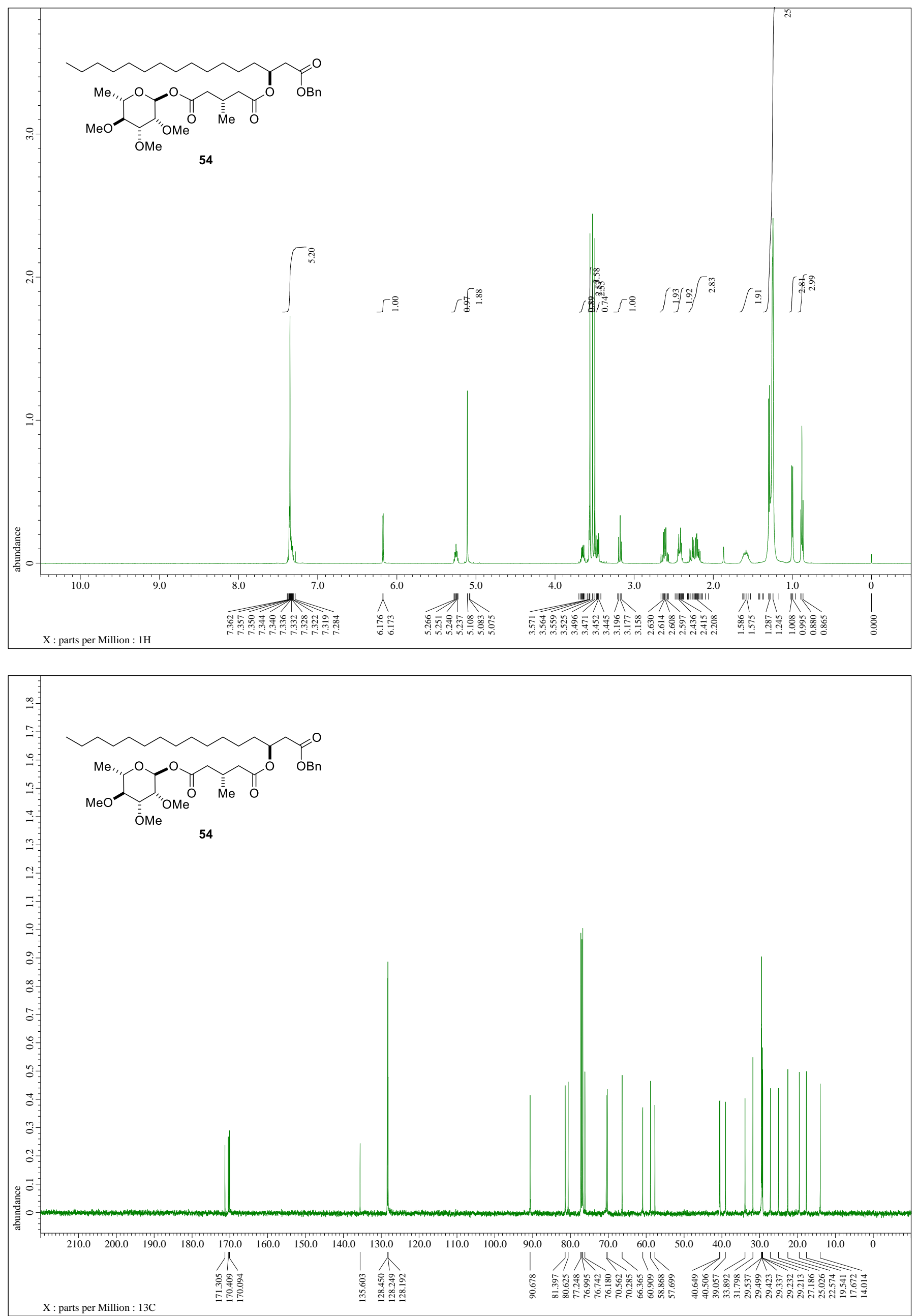

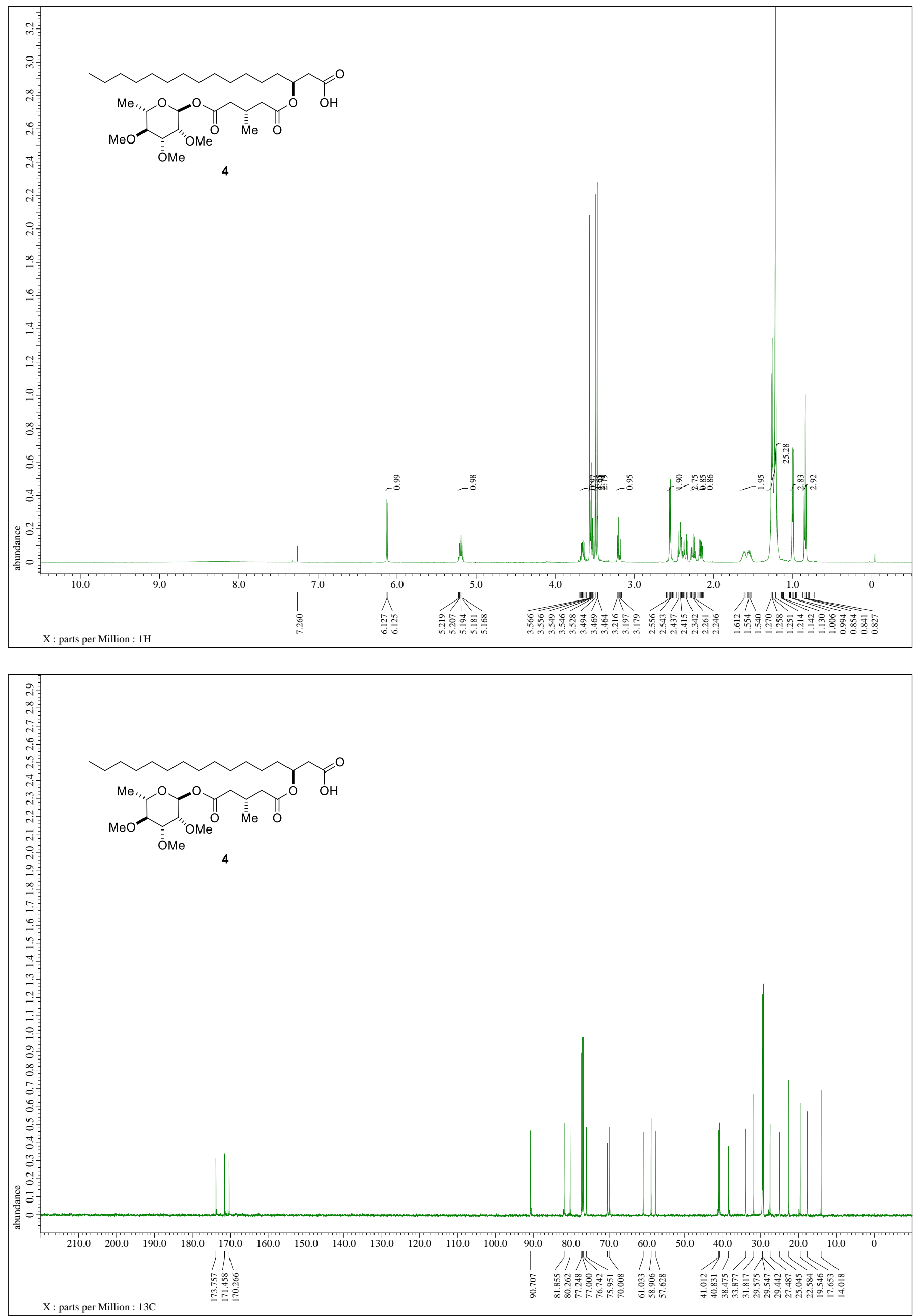

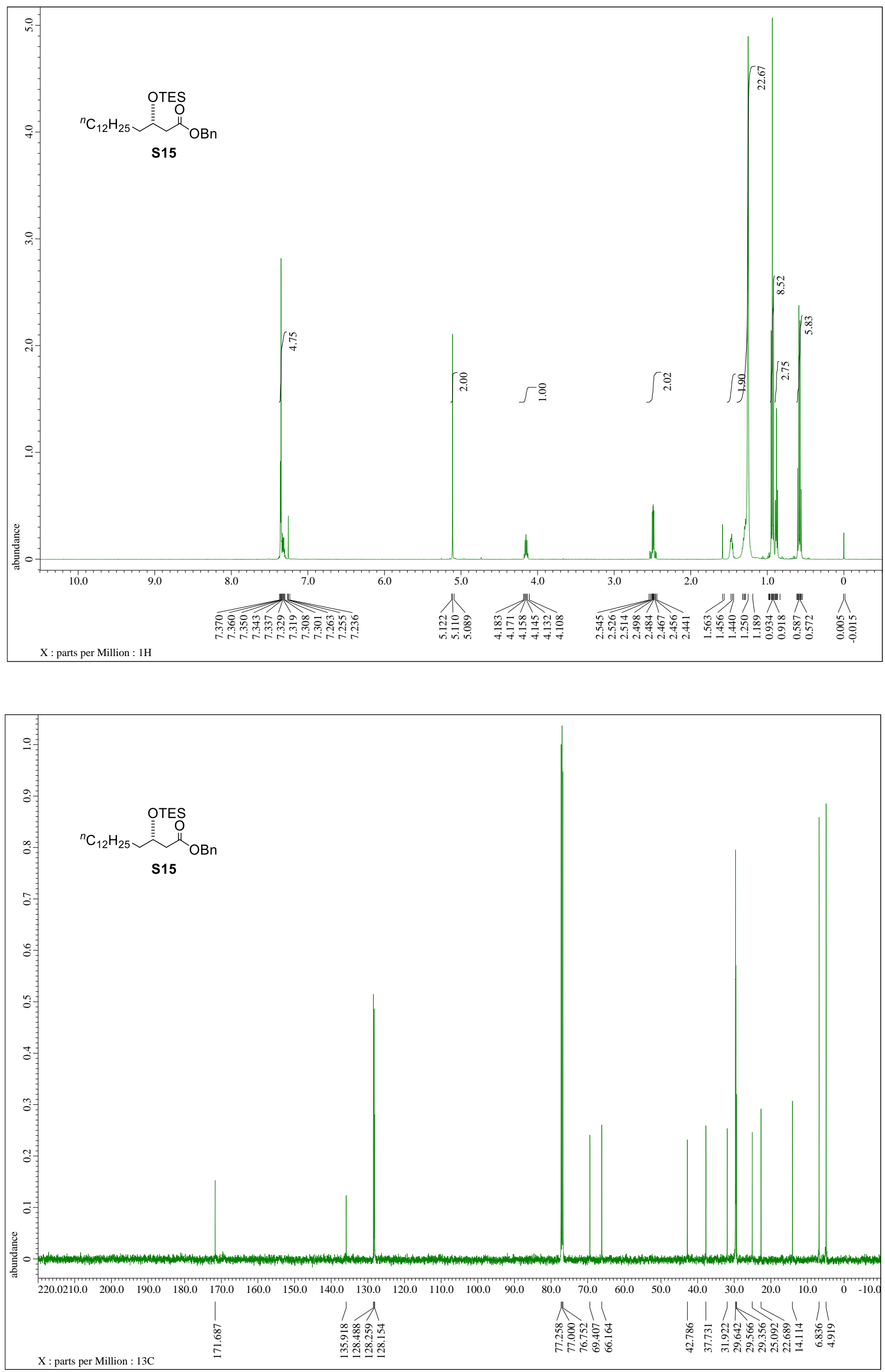

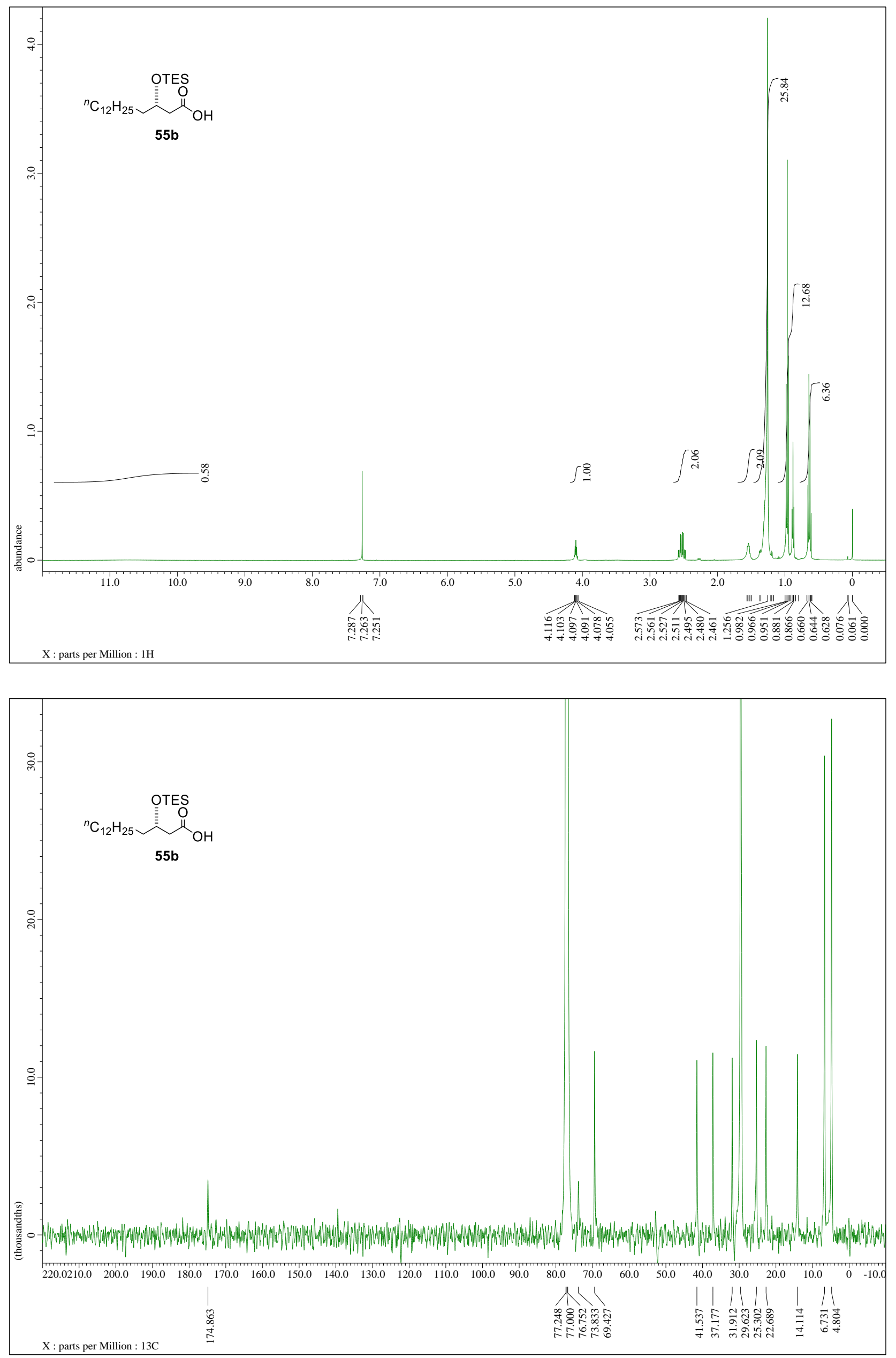

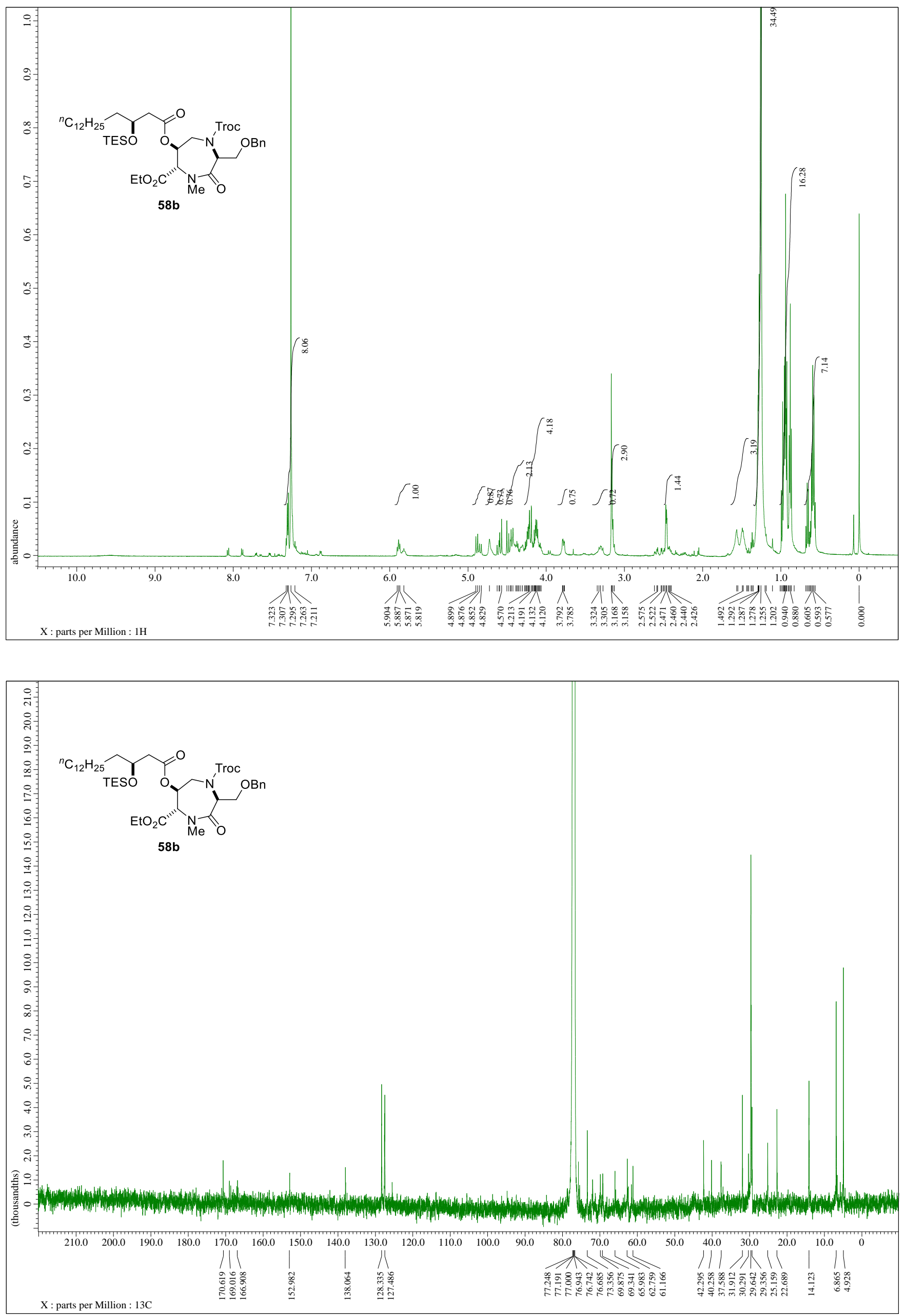

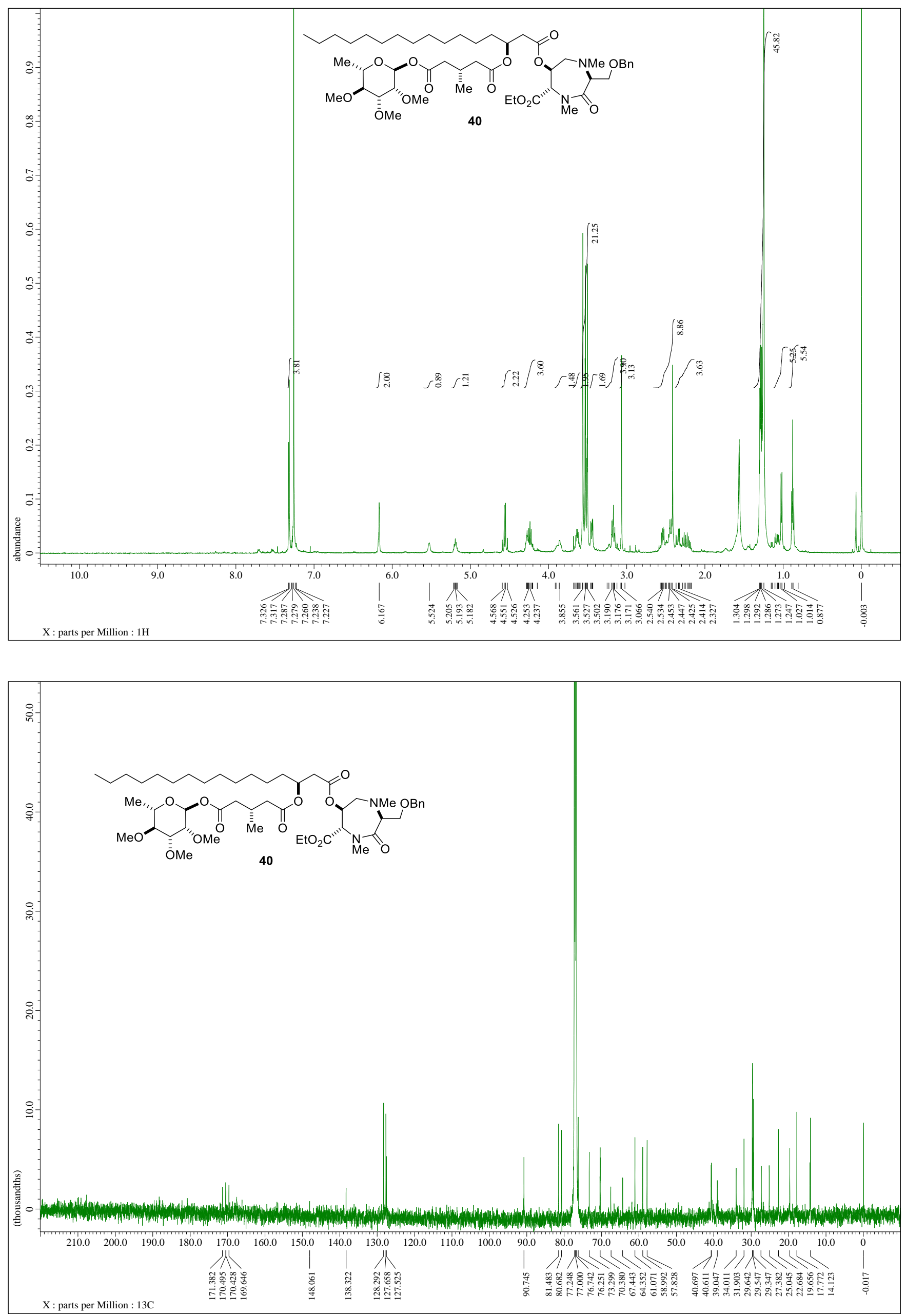

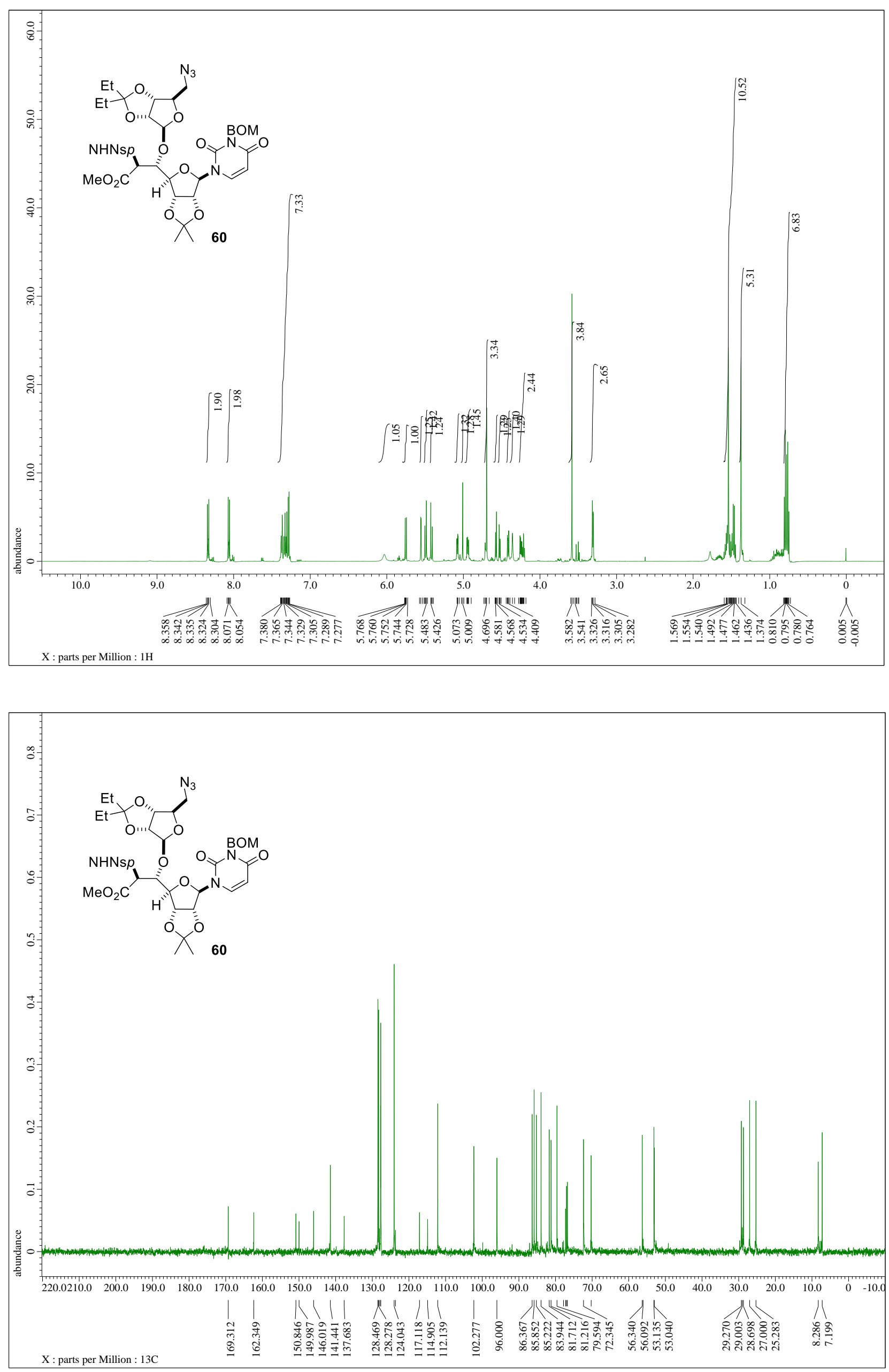

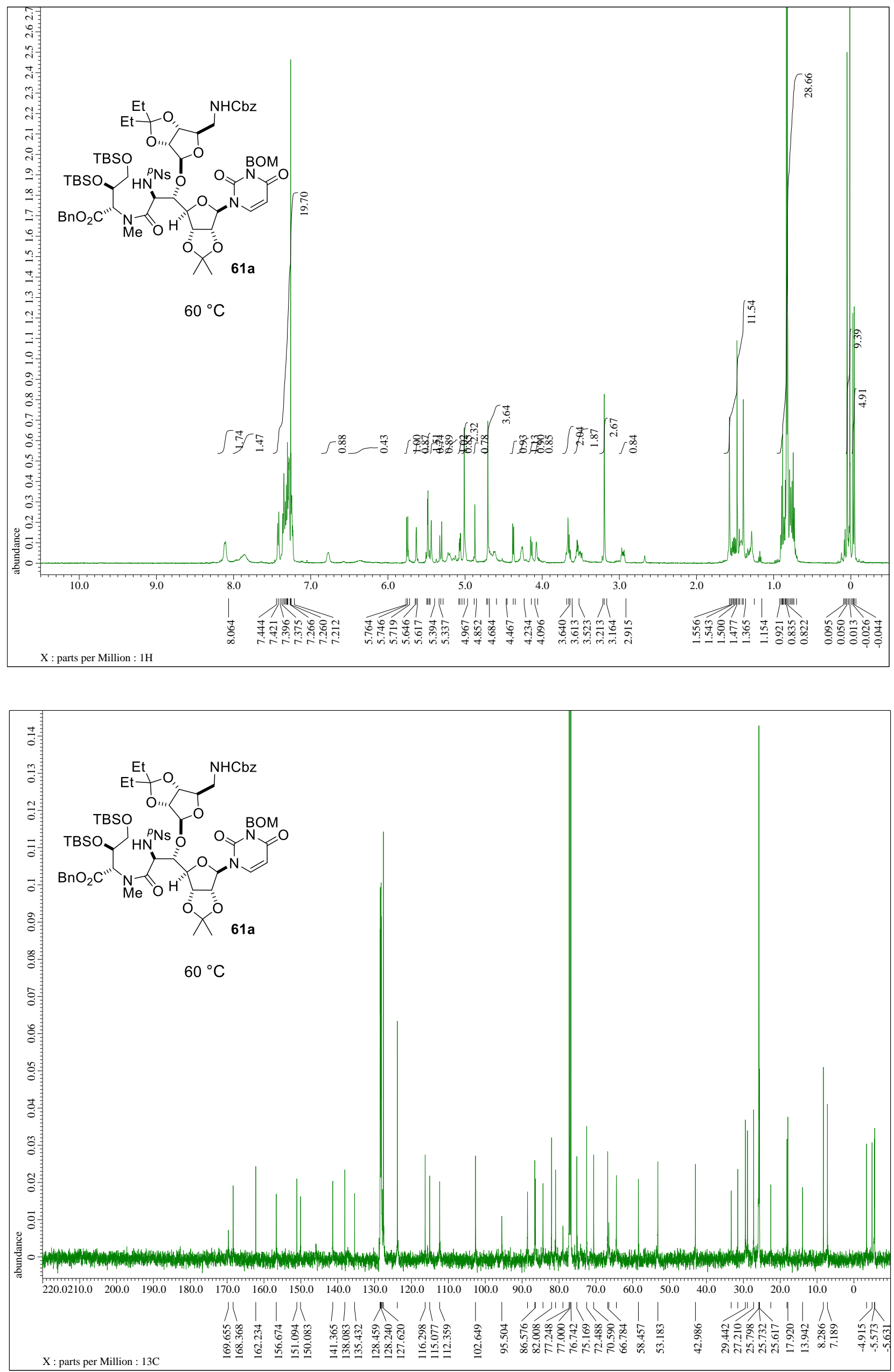

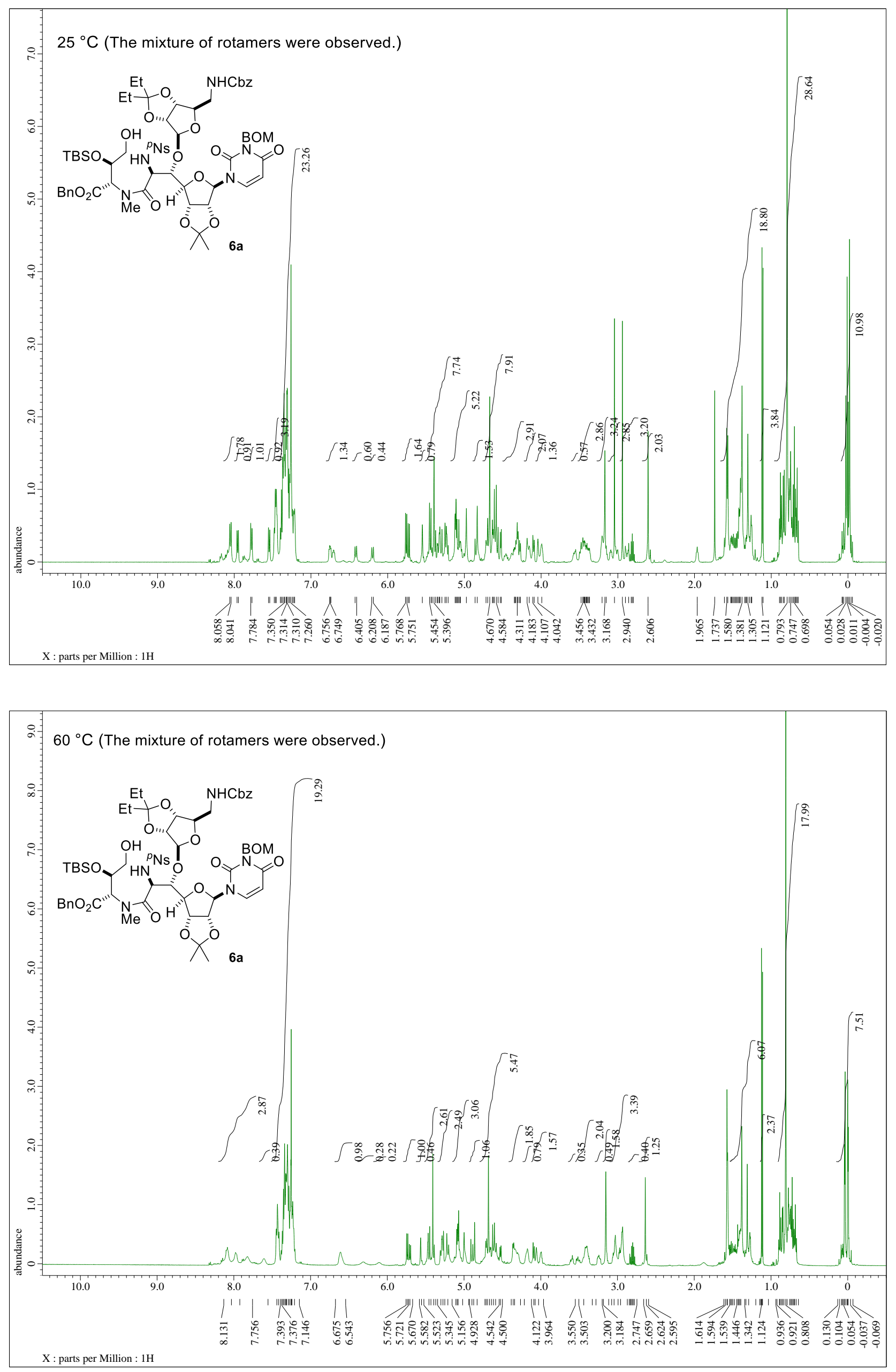


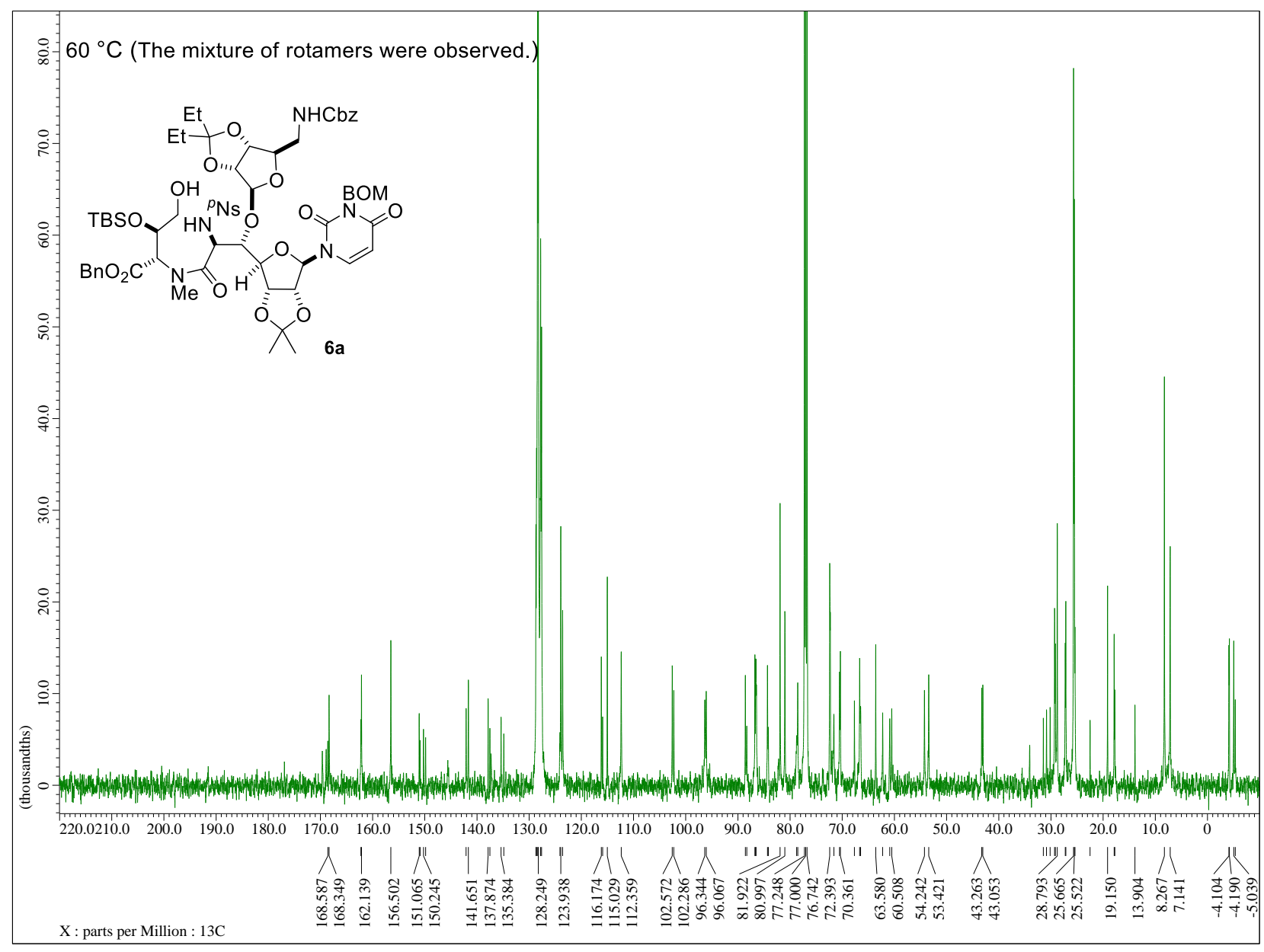



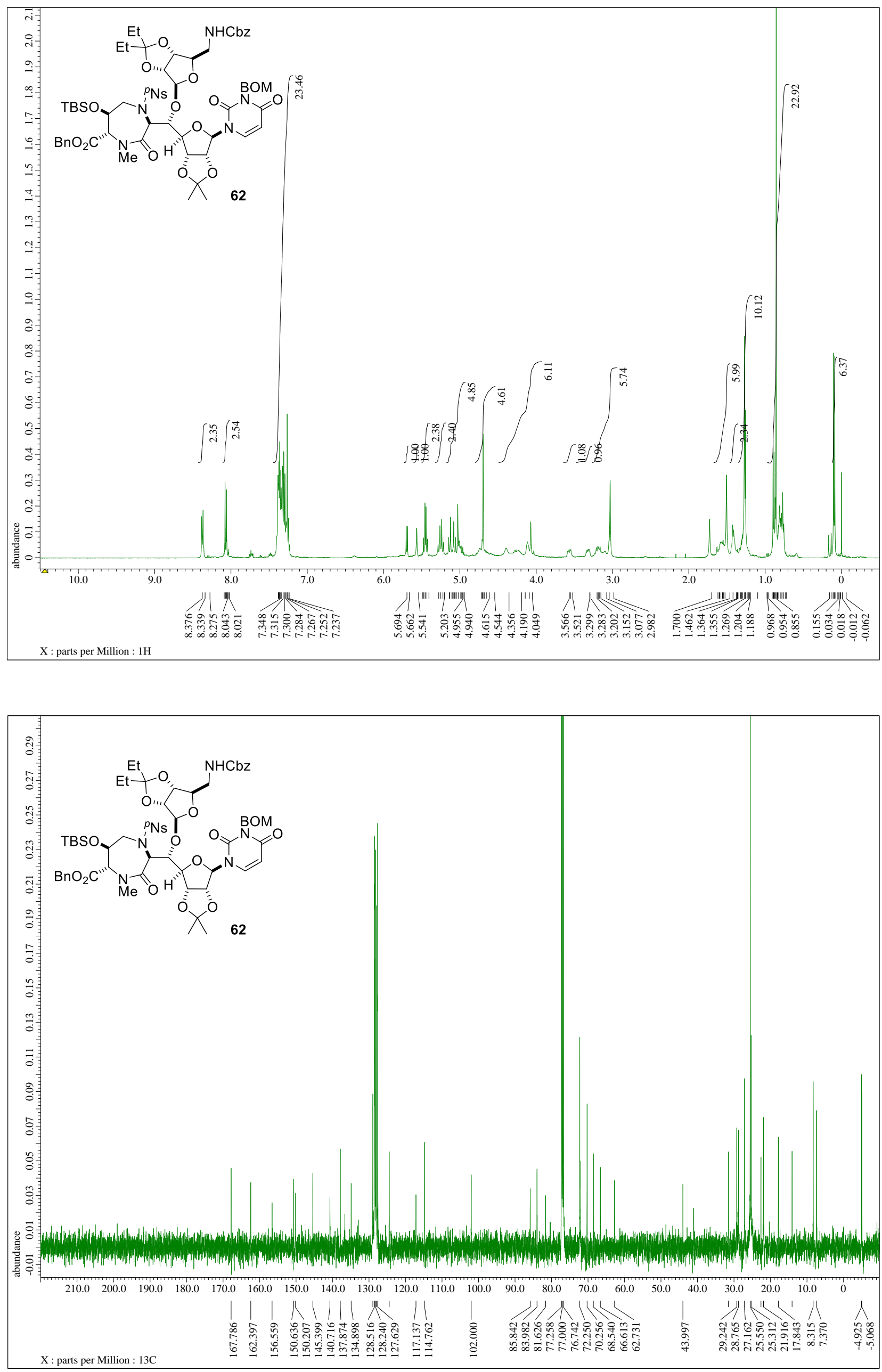

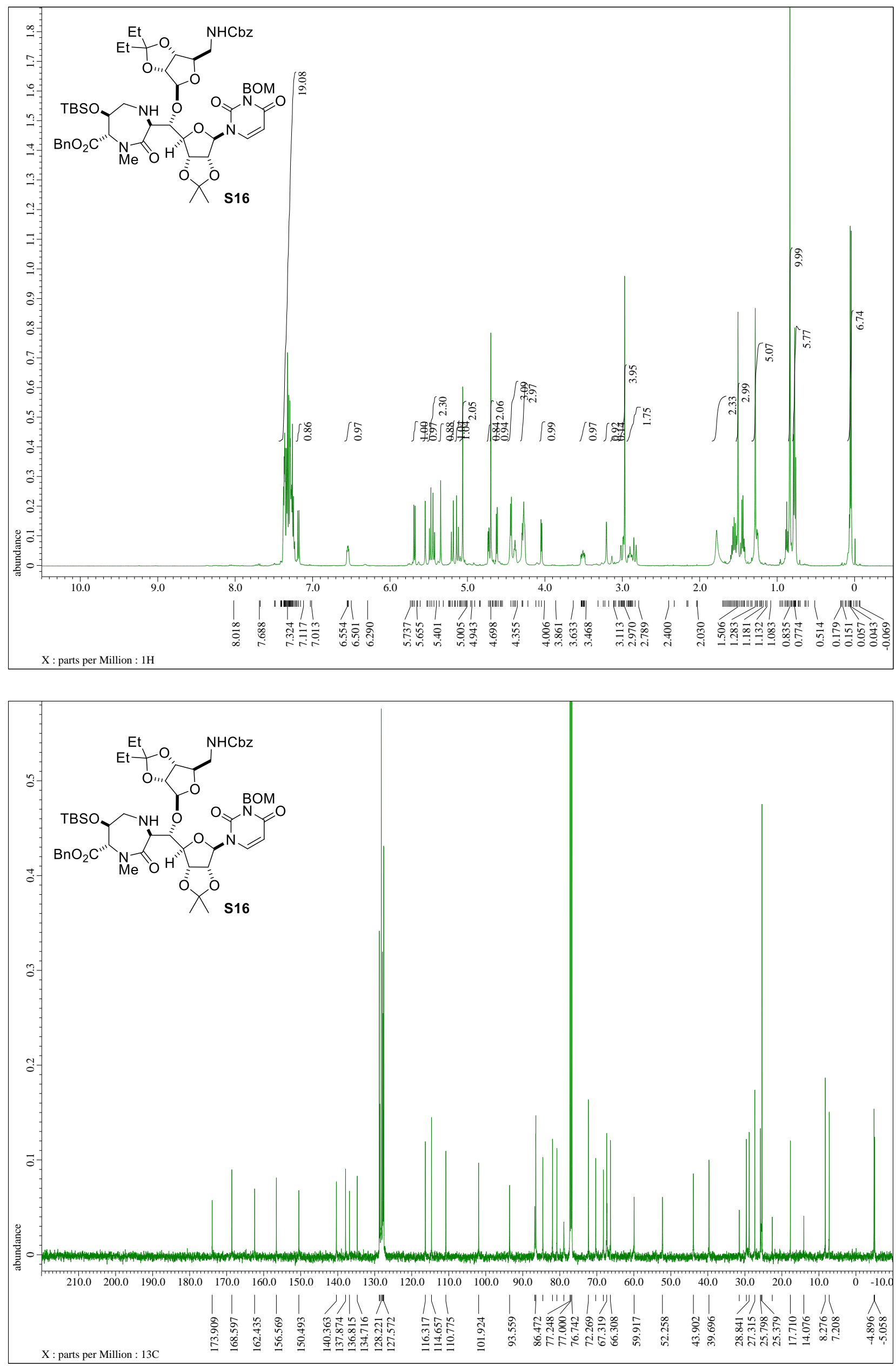

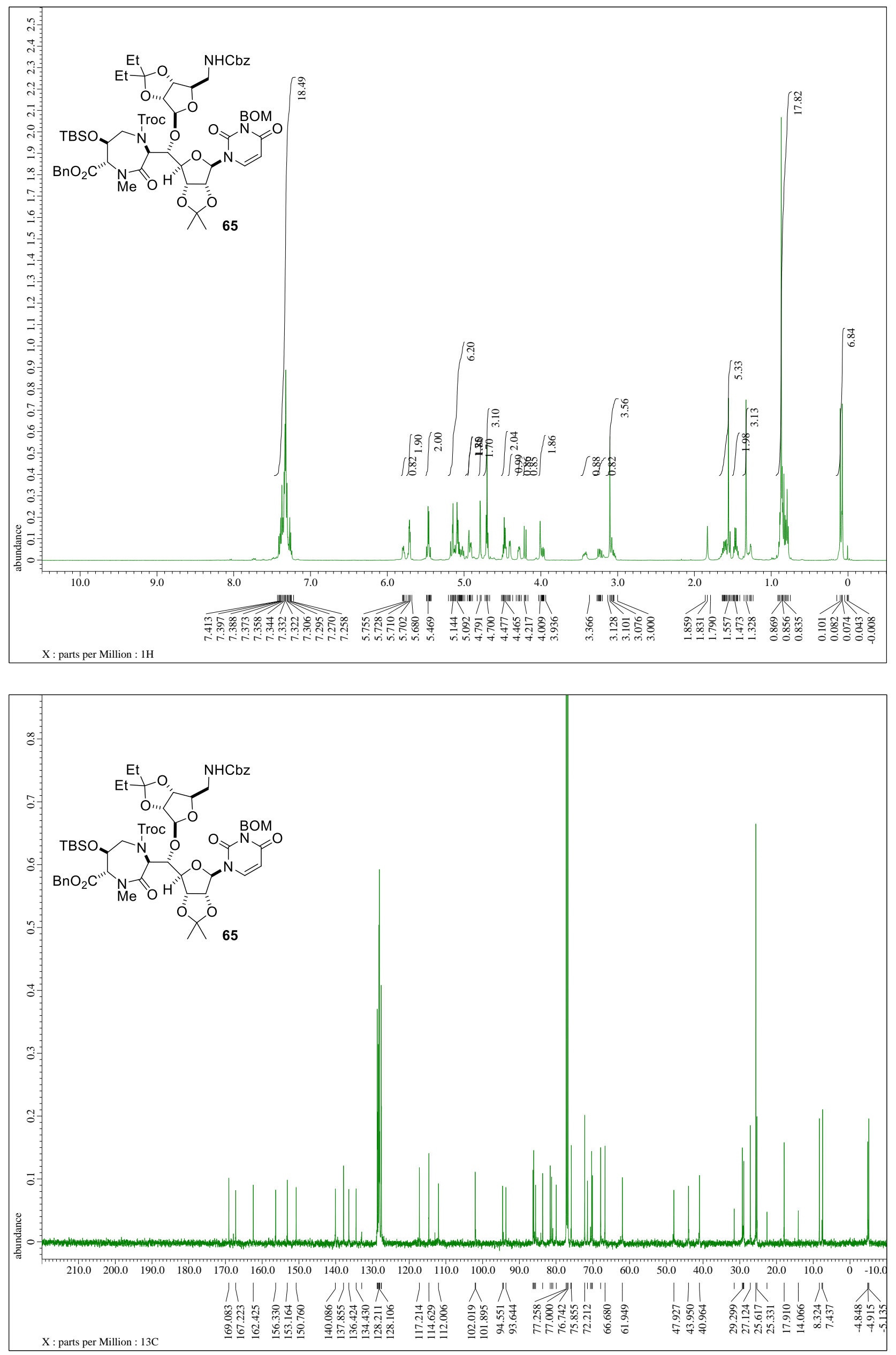

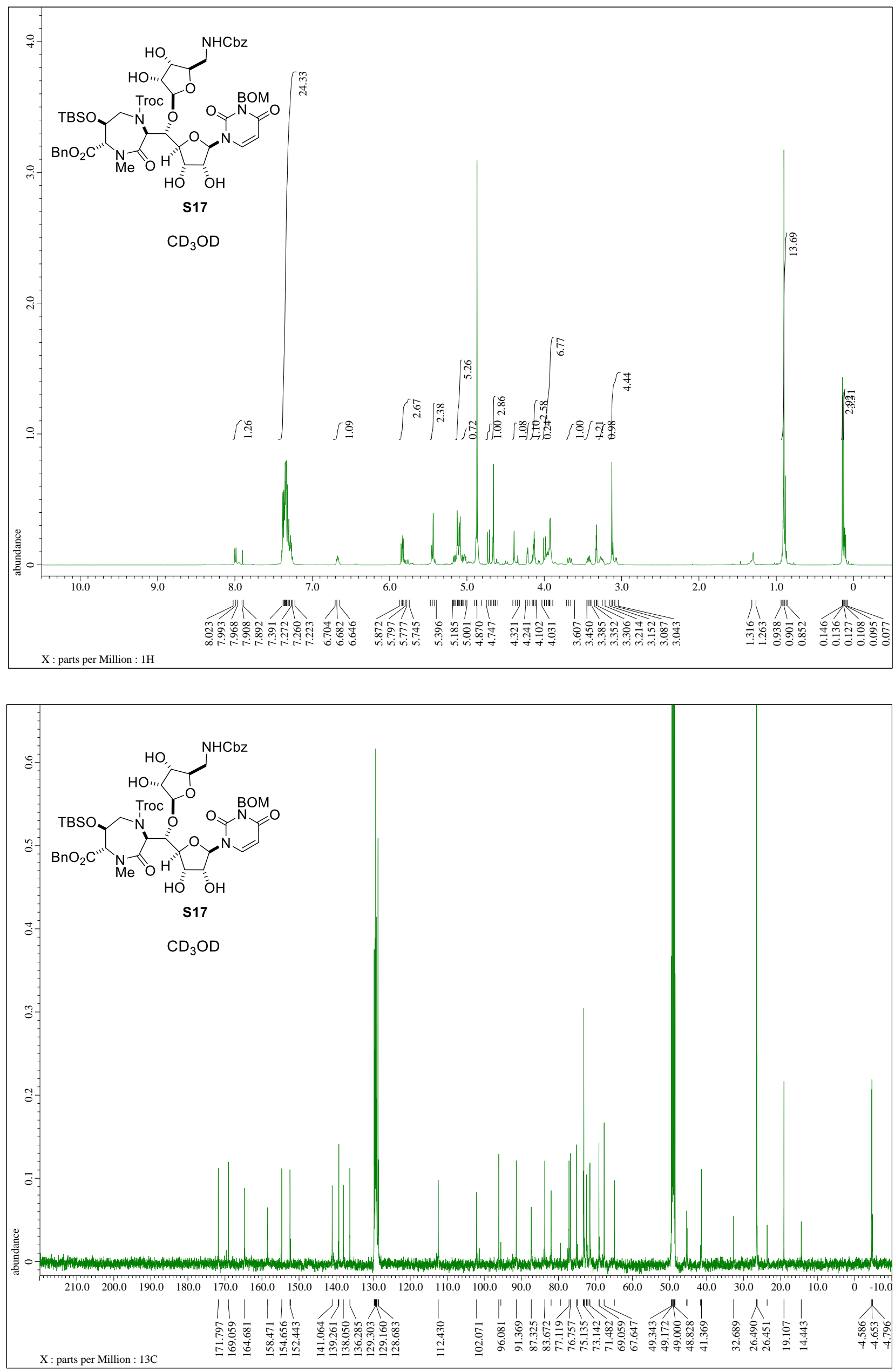

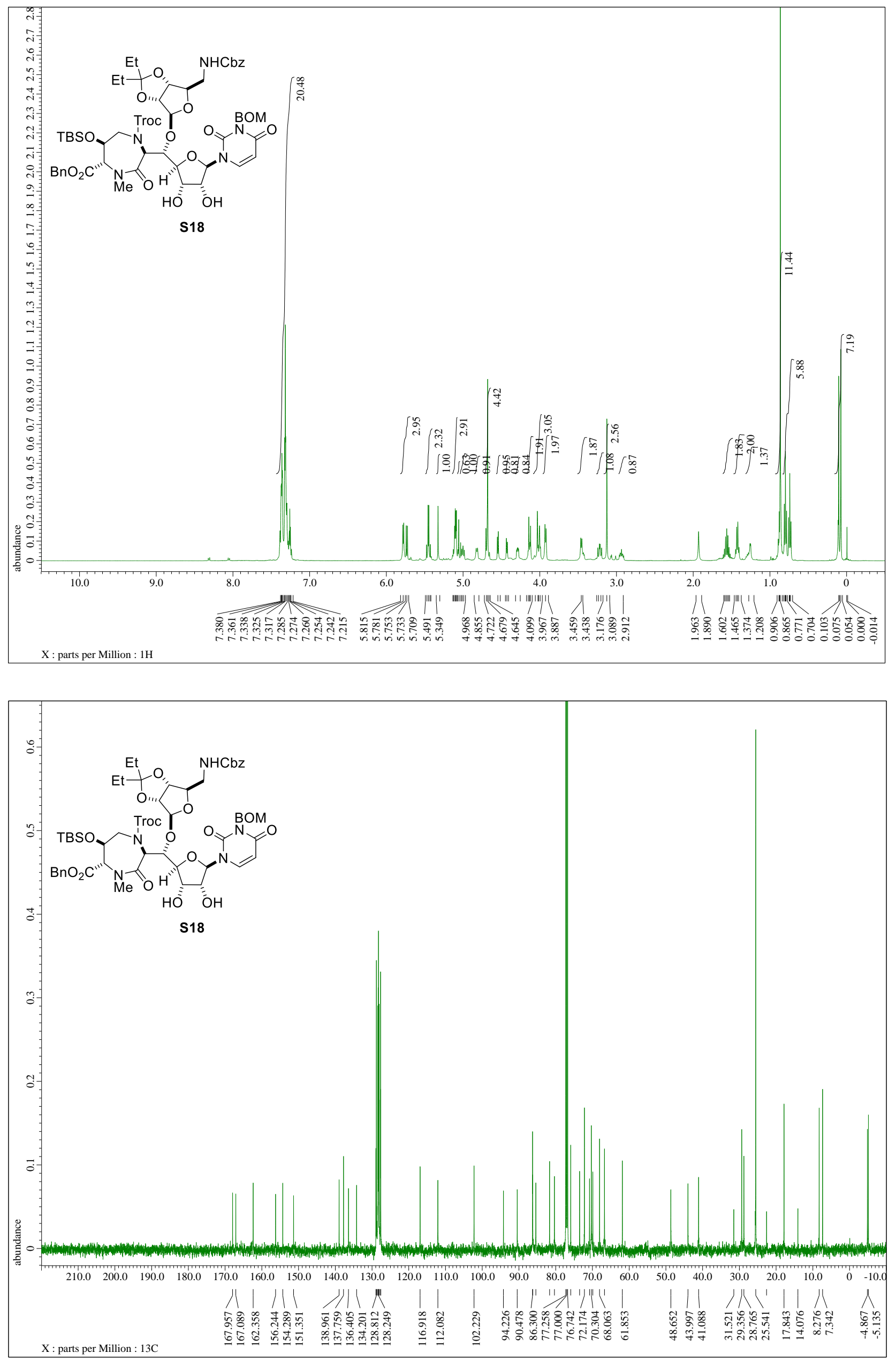

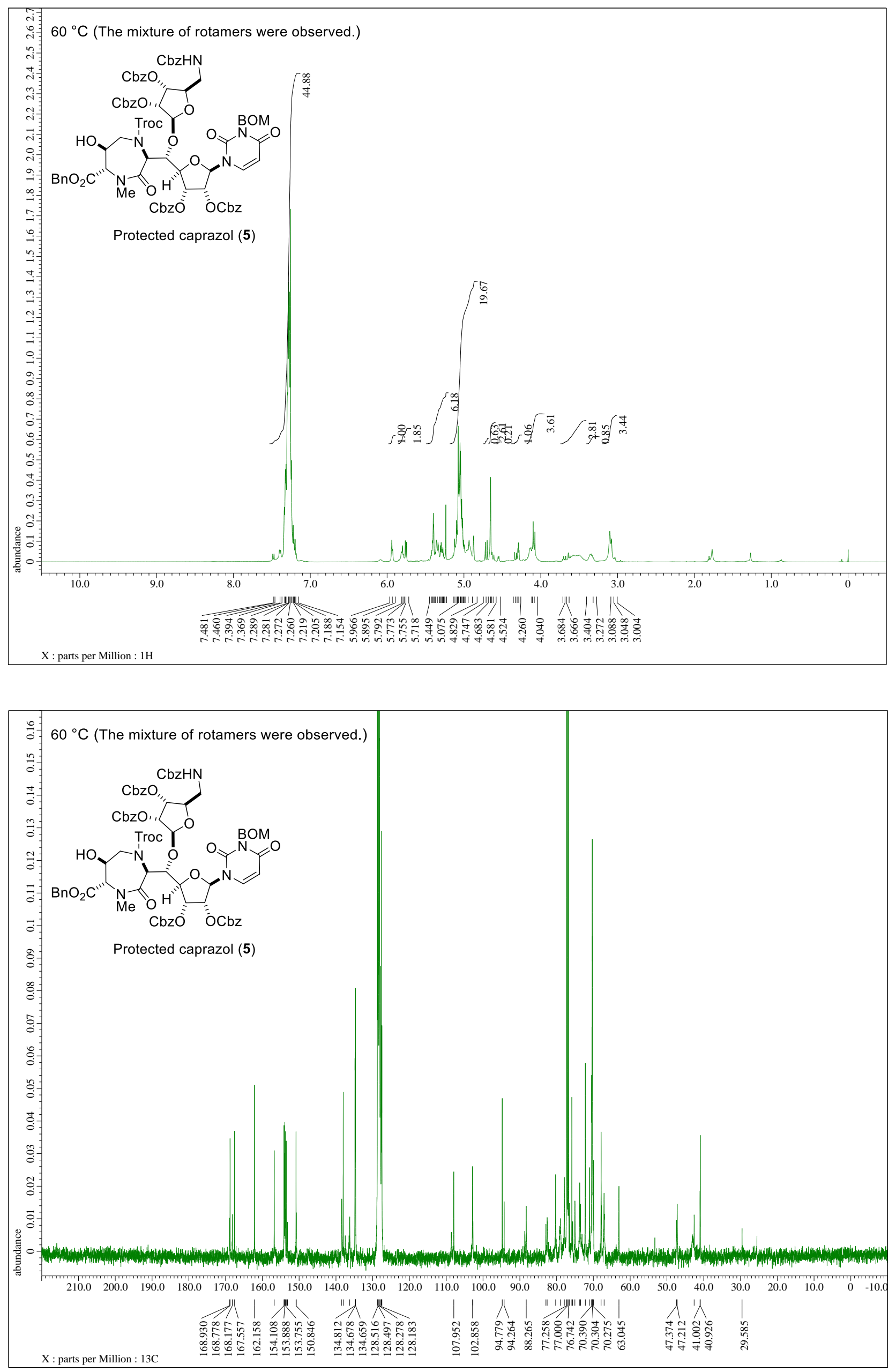

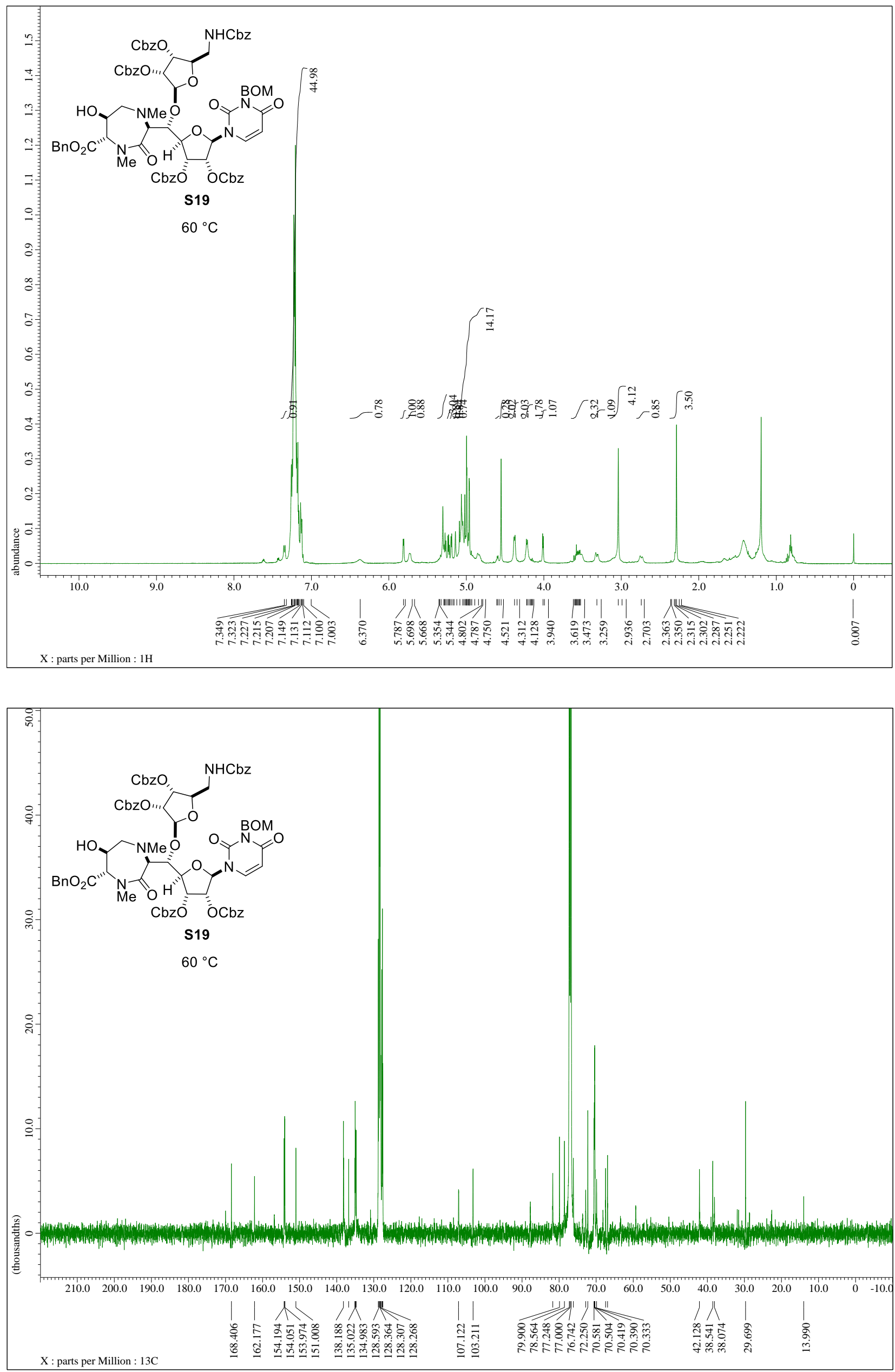

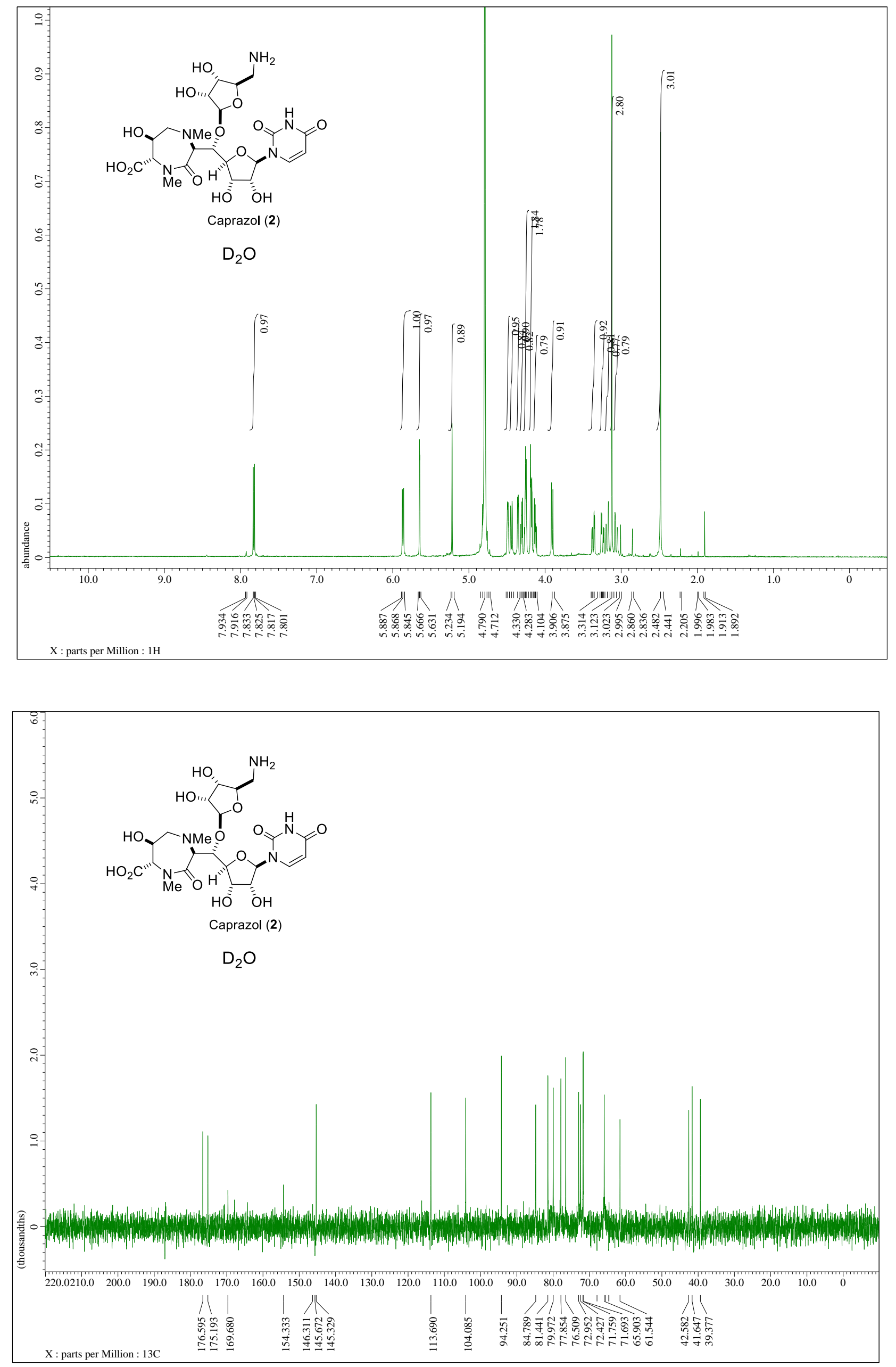

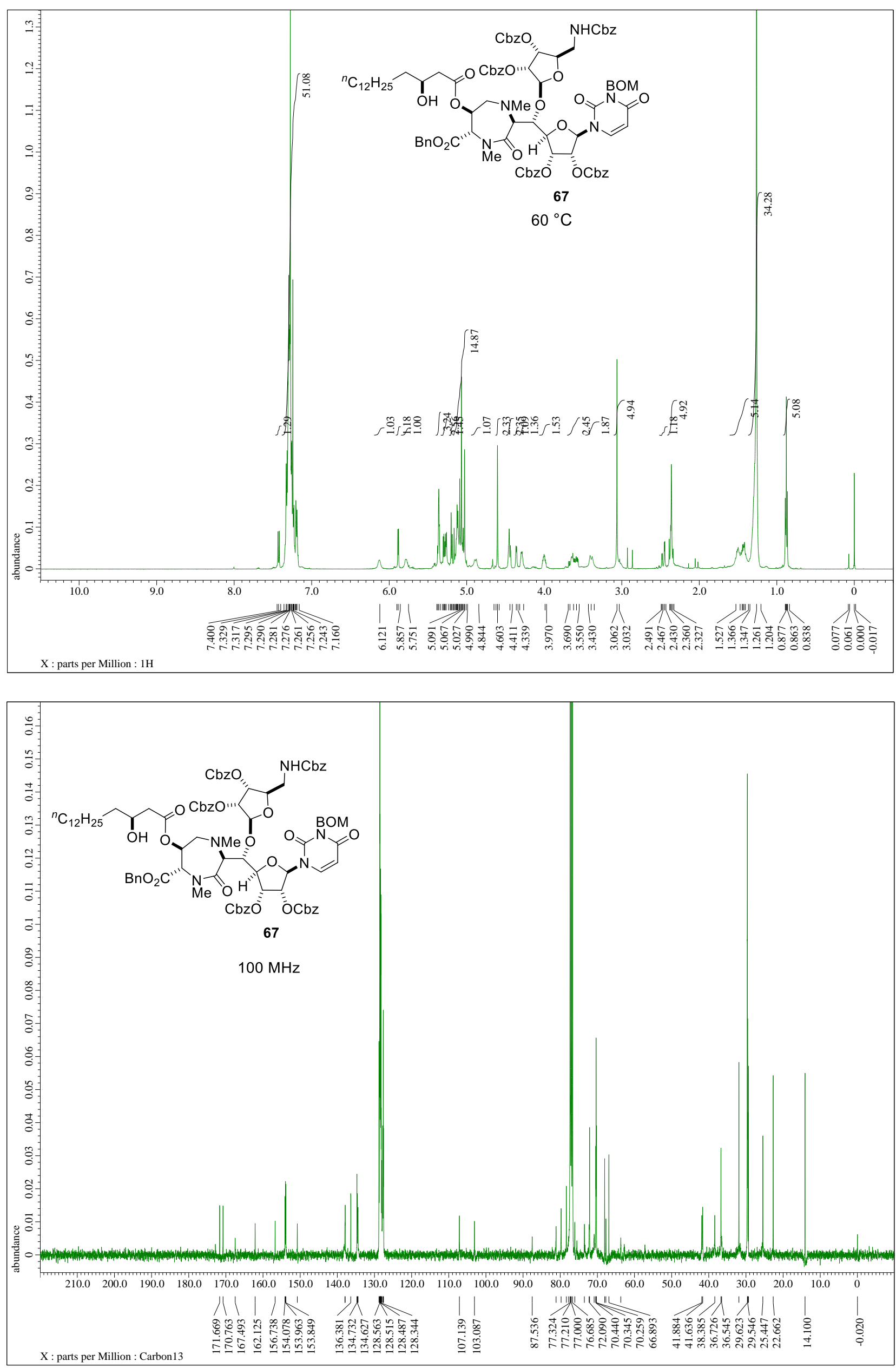

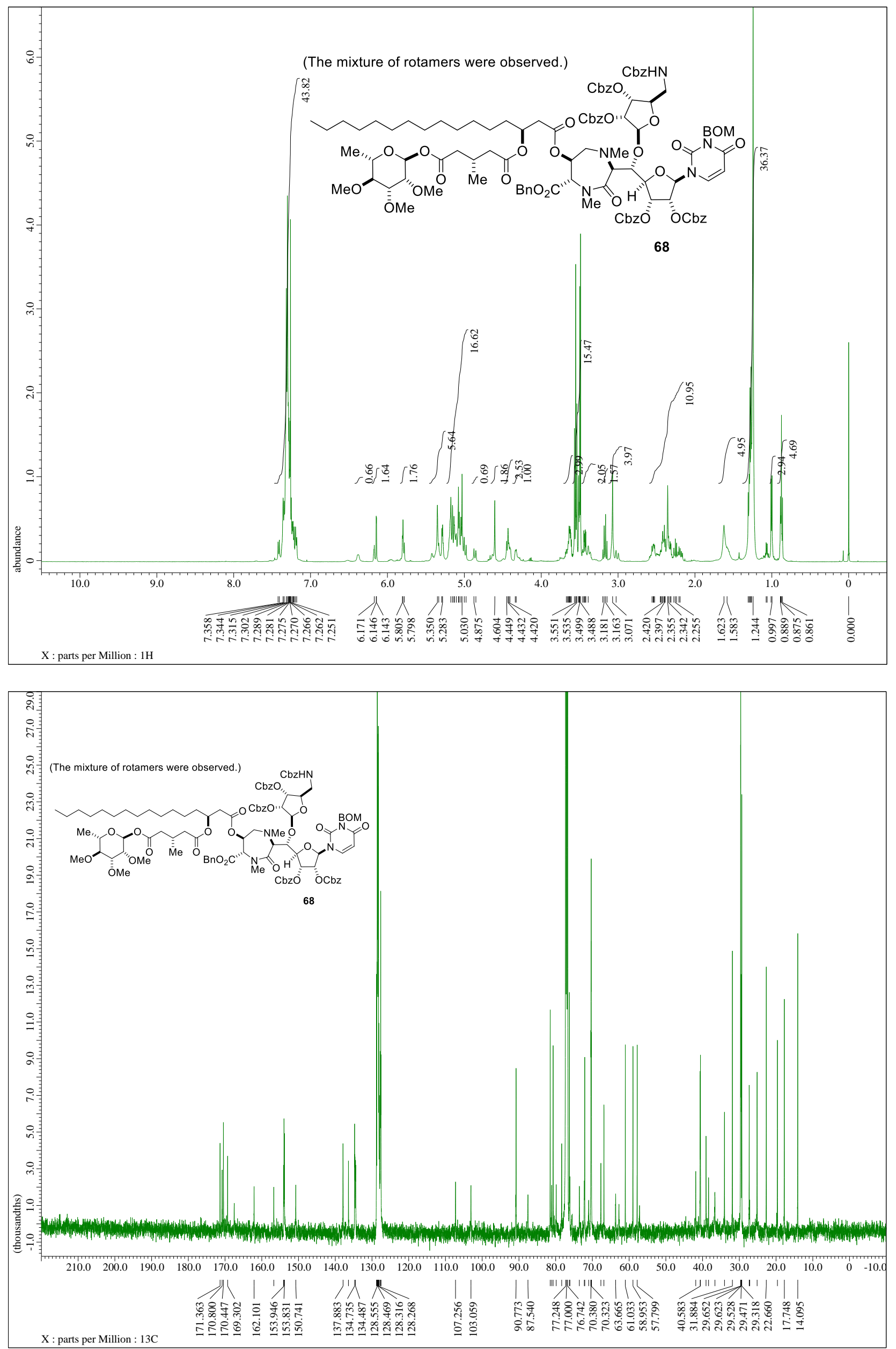

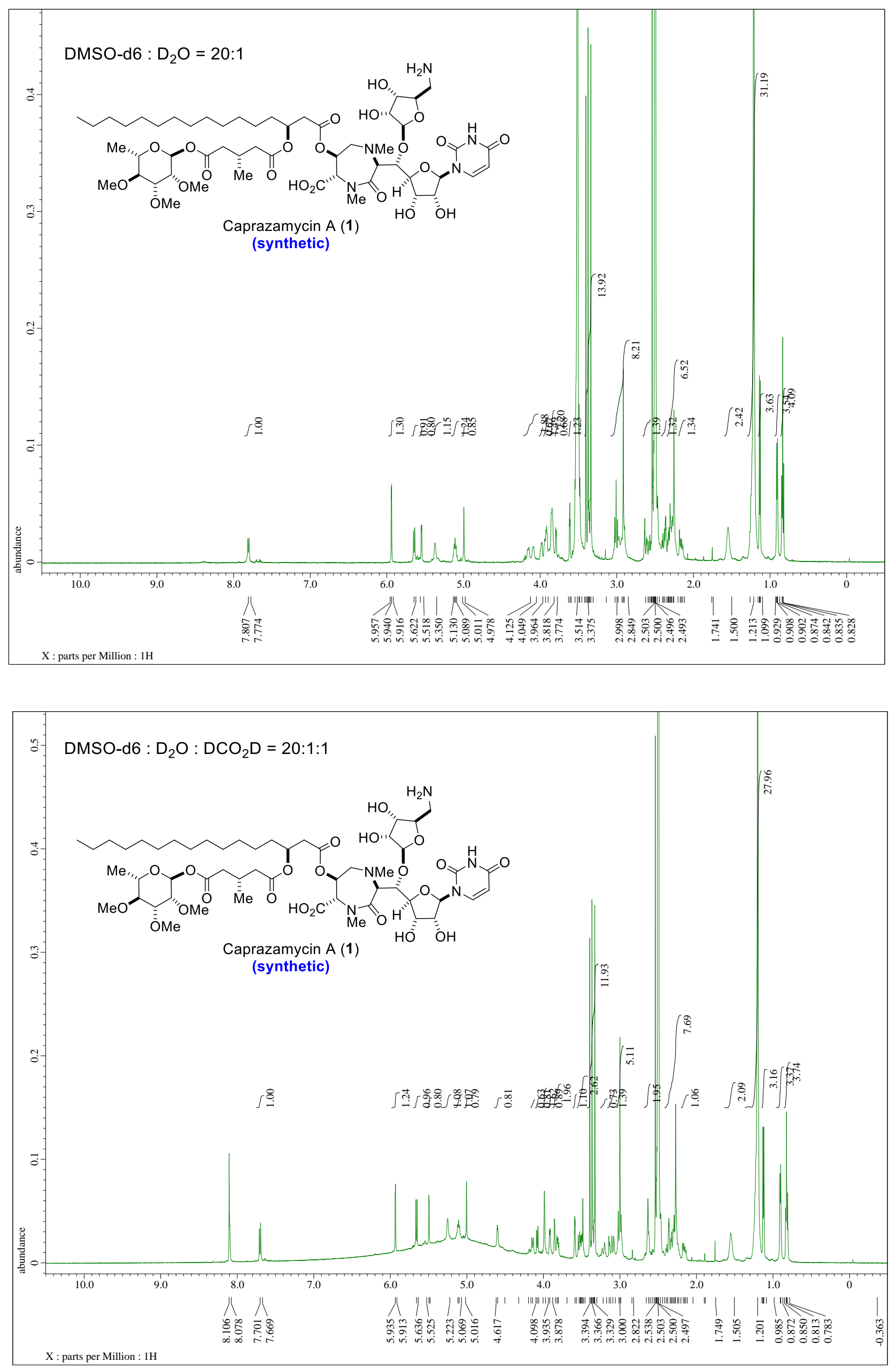

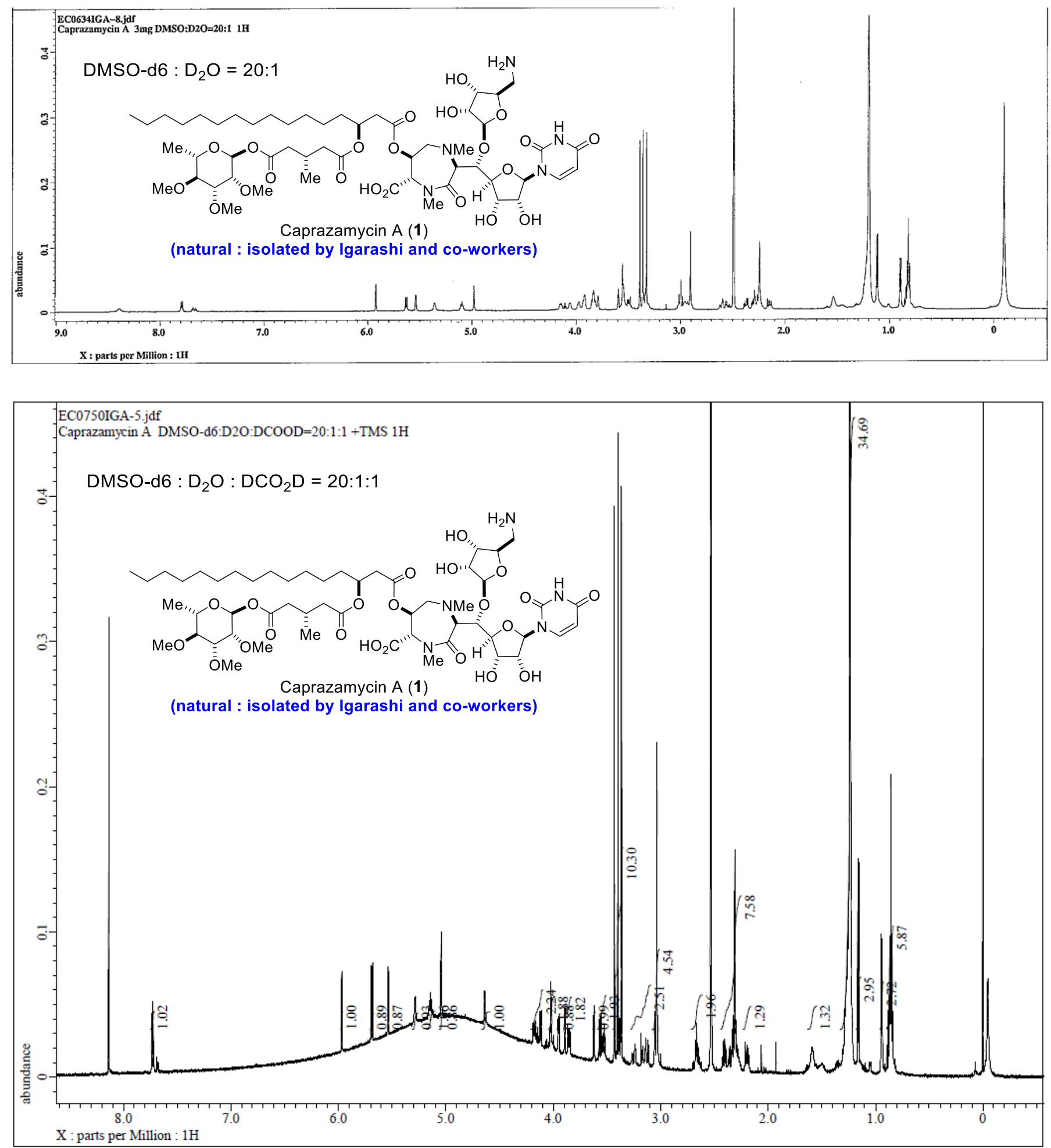


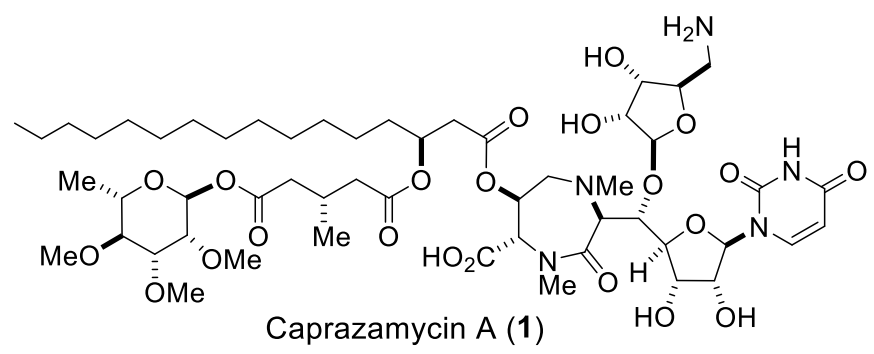

Natural (DMSO-d6/ $\left.\mathrm{D}_{2} \mathrm{O}=20: 1,600 \mathrm{MHz}\right)$

${ }^{1} \mathrm{H}$ NMR

isolated by Igarashi and co-workers

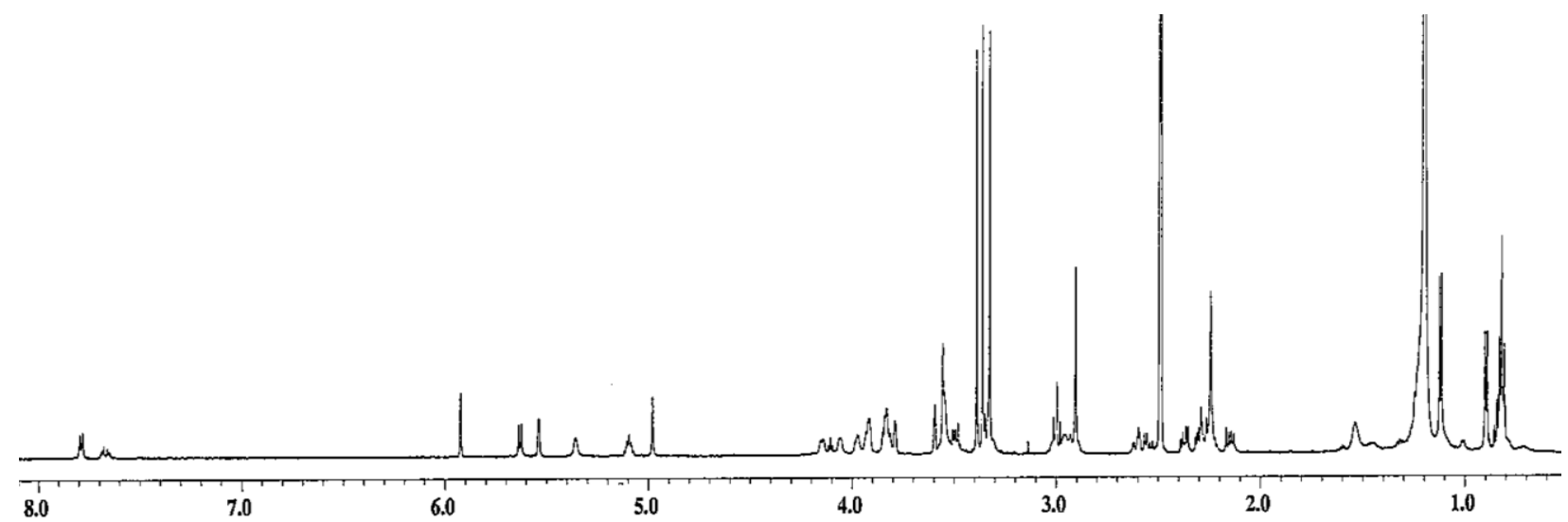

Synthetic (DMSO-d6/ $\left.\mathrm{D}_{2} \mathrm{O}=20: 1,500 \mathrm{MHz}\right)$

${ }^{1} \mathrm{H}$ NMR

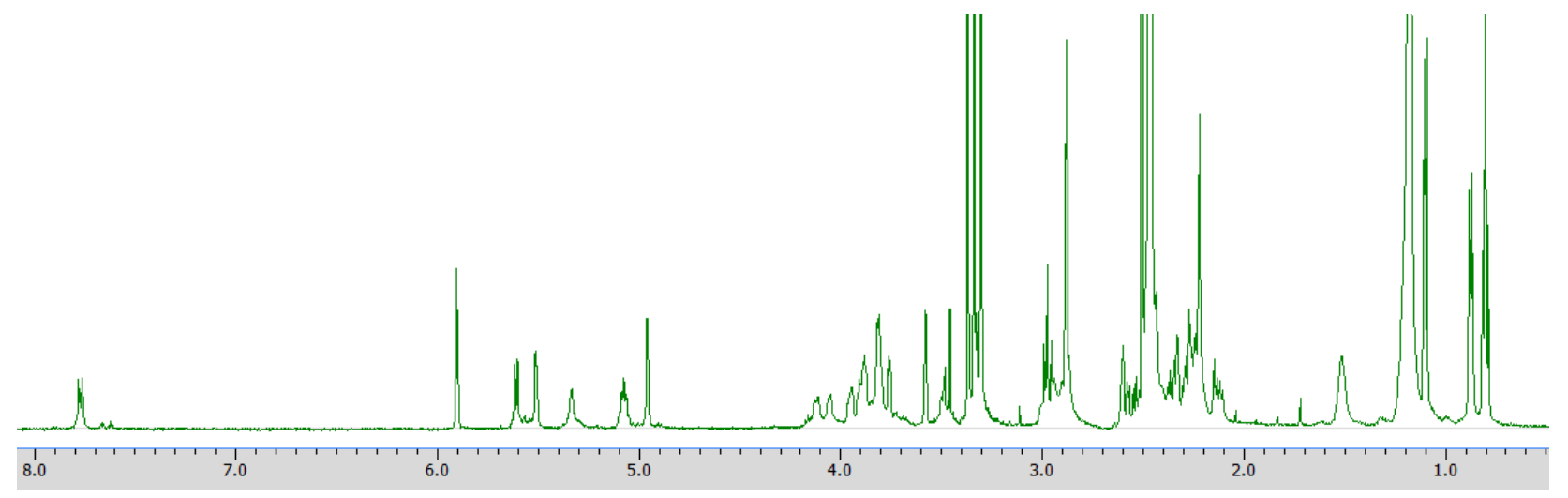

Figure 1. Comparison of the natural and synthetic ${ }^{1} \mathrm{H}$ NMR for caprazamycin A 

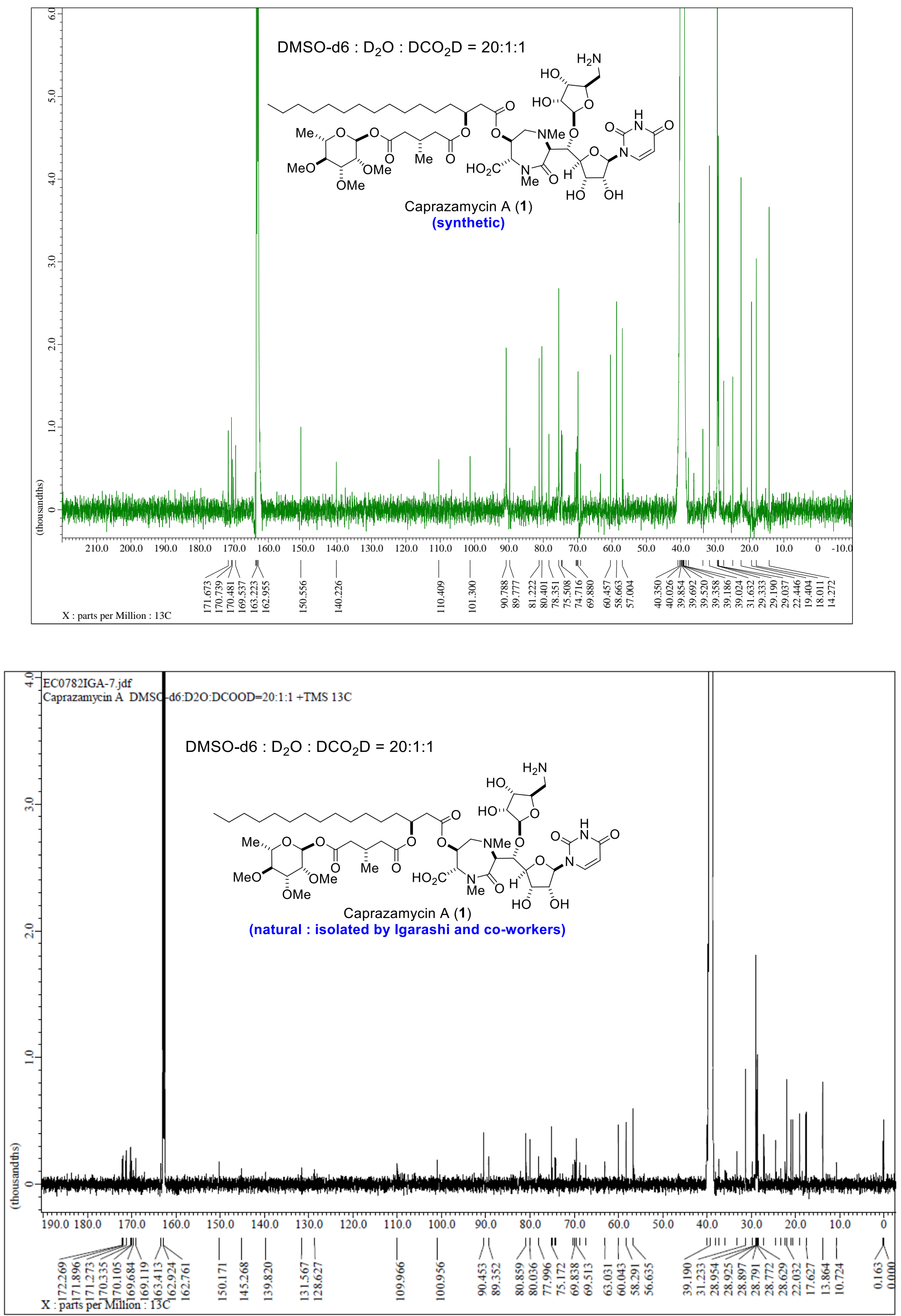


\section{Reference}

1. Wang, R.; Steensma, D. H.; Takaoka, Y.; Yun, J. W.; Kajimoto, T.; Wong, C.-H. A search for pyrophosphate mimics for the development of substrates and inhibitors of glycosyltransferases. Bioorg. Med. Chem. 1997, 5, 661.

2. Maeda, I.; Morishita, N.; Francom, M. J.; Robin, P. Synthesis of (S)-5-(4,5-Dihydroxypentyl)uracil from Pseudouridine and Clarification of Stereochemistry. J. Org. Chem. 1998, 63, 7539.

3. Silva, D. J.; Sofia, M. J. Novel carbohydrate scaffolds. Assembly of a uridine-mannose scaffold based on tunicamycin. Tetrahedron Lett. 2000, 41, 855.

4. Ogilvie, K. K.; Sadana, K. L.; Thompson, E. A.; Quilliam, M. A.; Westmore, J. B. The use of silyl groups in protecting the hydroxyl functions of ribonucleosides. Tetrahedron Lett. 1974, 33, 2861.

5. Spork, A. P.; Wiegmann, D.; Granitzka, M.; Stalke, D.; Ducho, C. Stereoselective Synthesis of Uridine-Derived Nucleosyl Amino Acids. J. Org. Chem. 2011, 76, 10083.

6. a) Mori, K.; Iwasawa, H. Stereoselective synthesis of optically active forms of $\delta$-multistriatin, the attractant for european populations of the smaller european elm bark beet. Tetrahedron 1980, 36, 87; b) Calderón, F.; Doyagüez, E. G.; Fernández-Mayoralas, A. Synthesis of Azasugars through a Proline-Catalyzed Reaction. J. Org. Chem. 2006, 71, 6258 .

7. Saito, S.; Bunya, N.; Inaba, M.; Moriwake, T.; Torii, S. A facile cleavage of oxirane with hydrazoic acid in dmf A new route to chiral $\beta$-hydroxy- $\alpha$-amino acids. Tetrahedron Lett. 1985, 26, 5309.

8. a) Roberts, T. C.; Smith, P. A.; Cirz, R. T.; Romesberg, F. E. Structural and Initial Biological Analysis of Synthetic Arylomycin A2. J. Am. Chem. Soc. 2007, 129, 15830; b) Tsukano, C.; Yokouchi, S.; Girard, A.-L.; Kuribayashi, T.; Sakamoto, S.; Enomoto, T.; Takemoto, Y. Platinum catalyzed 7-endo cyclization of internal alkynyl amides and its application to synthesis of the caprazamycin core. Org. Biomol. Chem. 2012, 10, 6074.

9. Hirano, S.; Ichikawa, S.; Matsuda, A. Total Synthesis of Caprazol, a Core Structure of the Caprazamycin Antituberculosis Antibiotics. Angew. Chem. Int. Ed. 2005, 44, 1854.

10. (a) Zhao, Y.; Truhlar, D. G. Density Functional for Spectroscopy: No Long-Range Self-Interaction Error, Good Performance for Rydberg and Charge-Transfer States, and Better Performance on Average than B3LYP for Ground States. J. Phys. Chem. A, 2006, 110, 13126. (b) Zhao, Y.; Truhlar, D. G. The M06 suite of density functionals for main group thermochemistry, thermochemical kinetics, noncovalent interactions, excited states, and transition elements: two new functionals and systematic testing of four M06-class functionals and 12 other functionals. Theor. Chem. Acc., 2008, 120, 215.

11. Chai, J.-D.; Head-Gordon, M. Long-range corrected hybrid density functionals with damped atom-atom dispersion corrections. Phys. Chem. Chem. Phys., 2008, 10, 6615.

12. Austin, A.; Petersson, G.; Frisch, M. J.; Dobek, F. J.; Scalmani, G.; Throssell, K. A Density Functional with Spherical Atom Dispersion Terms. J. Chem. Theory Comput. 2012, 8, 4989.

13. (a) Gonzalez, C.; Schlegel, H. B. An improved algorithm for reaction path following. J. Chem. Phys. 1989, 90, 2154. (b) Gonzalez, C.; Schlegel, H. B. Reaction path following in mass-weighted internal coordinates. J. Phys. Chem. 1990, 94, 5523.

14. (a) Miertuš, S.; Scrocco, E.; Tomasi, J. Electrostatic interaction of a solute with a continuum. A direct utilizaion of $\mathrm{AB}$ initio molecular potentials for the prevision of solvent effects. Chem. Phys. 1981, 55, 117. (b) Mennucci, B.; Tomasi, J. Continuum solvation models: A new approach to the problem of solute's charge distribution and cavity boundaries. J. Chem. Phys. 1997, 106, 5151. (c) Cammi, R.; Mennucci, B.; Tomasi, J. Fast Evaluation of 
Geometries and Properties of Excited Molecules in Solution: A Tamm-Dancoff Model with Application to 4-Dimethylaminobenzonitrile. J. Phys. Chem. A, 2000, 104, 5631. 